\title{
IDENTIFICATION, DOCUMENTATION \\ AND CONTROL OF BIOLOGICAL \\ CONTAMINATION IN MIDDLE \\ DISTILLATE FUEL
}

by

GLORIAH EMLY GRES HETTIGE

\author{
A thesis
}

submitted to the Victoria University of Wellington

in fulfilment of the

requirements for the degree of

Doctor of Philosophy

in Botany

Victoria University of Wellington

January 1987 
The present study was initiated under a contract with the New Zealand Defence Scientific Establishment to document the nature, extent and possible sources of microbial contamination of middle distillate fuel ('Dieso') held in storage tanks at Devonport, Auckland, and to study possible methods for control of such contamination.

Thirty-one fungal species and five bacteria were isolated during the period 1982-1984. The principal contaminants were the fungi Cladosporium resinae (the anamorph of Amorphotheca resinae Parbery), Penicillium corylophilum and Paecilomyces variotii. All three fungi produced dark mycelial mats at the water/diesel fuel interface in laboratory studies. Interactions between these fungi were observed. In the presence of Bushnel1-Haas mineral salts/diesel fuel phases Cladosporium resinae predominated while in seawater/diesel fuel phases Penicillium corylophilum predominated. All New Zealand and Australian isolates of $C$. resinae grew profusely in Bushnel1-Haas mineral salts/diesel fuel phases.

The biostatic/biocidal effects of chemicals on the predominant fungi in diesel fuel were studied in laboratory and field tests during 1984-1985. The most effective biocides in controlling C. resinae were benomyl, imazalil and Kathon 886. Imazalil had no effect on Paecilomyces variotii but when used in combination with benomyl a synergistic effect occurred at $100 \mathrm{ppm}$. Biobor JF, DEGME and EGME performed poorly in laboratory tests regardless of the amount of water present, but gave temporary inhibition of $C$. resinae in the field tests. Isolates from tanks treated with Biobor JF and DEGME grew well in the presence of these compounds in the laboratory. DML-7 and Proxel AS inhibited $C$. resinae and Penicillium spp. in both laboratory and field tests at a high dose of 300 ppm but were less effective against $P$. variotii. The effects of the biocides on engine performance and carbon deposits on engine components were studied.

Recommendations for control of microbiological contamination of stored diesel fuel are given. In electron microscope studies no difference was observed in the intracellular structures between jet and diesel fuel isolates of $C$. resinae and the non-hydrocarbon utilizing Cladosporium cladosporioides. 


\section{ACKNOWLEDGEMENTS}

I wish to thank:

My supervisors Dr. J.E. Sheridan and Professor J.K. Heyes for many helpful discussions and for their critical reading of the manuscript.

The staff of the Defence Scientific Establishment, Auckland, for their help and assistance especially Mr. H. Levinsohn, Senior Metallurgist and $\mathrm{Mr}$. M. Cavanaugh.

The Ministry of Defence for financing this work, and this is greatly appreciated.

Technical staff of the Botany Department for their help.

Dr. R. Wilson, Chemistry Division of DSIR, Wellington for the chemical analysis of a biocide.

Mr. R. Renner for the assistance with statistical analysis.

Mr. C. Chinnaiyah for his invaluable help with the computer graphics.

Dr. A. Bell for friendly advice and reading of the manuscript.

Many friends who have provided constant encouragement towards the completion of the thesis.

My husband, Dayandra Hettige, for his love, patience, encouragement and moral support at all times during this project. I owe much to him.

My parents who have provided constant encouragement. 
ABSTRACT

ACKNOWLEDGEMENTS

LIST OF FIGURES

LIST OF TABLES

CHAPTER 1 GENERAL INTRODUCTION AND AIMS OF STUDY

1.1 Studies on microbiological contamination of hydrocarbon fuels

1.1 .1

Early reports of microbial attack

1.1 .2

Morphology of Cladosporium resinae

1.2

Control of microbiological contamination in hydrocarbon fuel.

1.2.1 Criteria for the choice of fuel biocides

1.3 Aims of study.

$\underline{\text { PART } 1}$

STUDIES ON MICROBIOLOGICAL CONTAMINATION OF DIESEL FUEL

CHAPTER 2 MATERIALS AND METHODS

2.1 Sampling of storage tanks 17

$\begin{array}{lll}2.1 .1 & \text { Tank description } & 17\end{array}$

2.1.2 Collection of samples 20

2.1.3 Sampling methods 21

$\begin{array}{lll}2.1 .4 & \text { Sampling pattern } & 21\end{array}$

2.2 Detection of micro-organisms in the fuel samples 22

$2.2 .1 \quad$ Culture media 22

2.2.2 Membrane filtration 22 
2.2.3 Direct observation

2.2.4 Pour plate method

2.2.5 Spread plates 24

2.3 Detection of micro-organisms in air and soil

2.3.1 Dilution plating

$2.4 \quad$ Identification of micro-organisms

2.5 Laboratory studies using fungi isolated from diesel fuel

2.5.1 Growth studies

2.5.2 Effect of creosote on fungal growth

2.5.3 Shaking and transport effects on spore viability in fuel

Fine structure studies on hydrocarbon-utilizing

2.6 .1 fungi.

Light microscopy study

2.6 .3

Scanning electron microscopy (SEM)

Transmission electron microscopy (TEM)

2.7 Determination of water in diesel fuel samples

2.8 Meteorological records

CHAPTER 3 EXPERIMENTAL RESULTS : SECTION I - FIELD STUDIES

3.1 Micro-organisms occurring in diesel fuel

3.2 Comparison of isolation methods

3.3 Comparison of container type used for collecting samples

3.4 Effect of time of collection and testing of samples on the recovery of Cladosporium resinae 
3.5 Sources of fuel contamination

3.6 Discussion

CHAPTER 4 EXPERIMENTAL RESULTS : SECTION II - LABORATORY STUDIES

4.1 Growth studies

4.1.1 Single species culture

4.1.2 Growth in mixed cultures

4.2 Creosote tolerance studies

4.3 Effects of shaking and transport on spore viability

4.4 Morphology and ultrastructure studies

4.5 Discussion

$\underline{\text { PART } 2}$

CONTROL OF MICROBIOLOGICAL CONTAMINATION IN DIESEL FUEL

\section{CHAPTER 5 MATERIALS AND METHODS}

5.1 Laboratory studies on fuel biocides

5.1 .3

The effects of biocides on fungal growth and survival in $\mathrm{BH} / \mathrm{diesel}$ phases

5.1 .4

Large volume liquid fuel : biocide tests

5.1 .5

Intermittent exposure of the three common fuel fungi to biocides in diesel fuel

Field evaluation of the fuel biocides 
5.2.3.1 Engine performance and combustion chamber

5.2 .3 .2 components

5.2.4 Water and biocide content of fuel samples

5.2 .4 .1

Determination of water content

5.2 .4 .2

Determination of partition coefficient

CHAPTER 6 EXPERIMENTAL RESULTS

6.1 SECTION I - Laboratory studies of biocide effects.

6.1 .1

6.1 .2 Agar/diesel slide technique

6.1 .3

Poison plate test

The effects of biocides on fungal growth and

6.1 .4 survival in $\mathrm{BH} / \mathrm{diesel}$ phases

6.1 .5

Large volume liquid fuel : biocide tests

Intermittent exposure of the three common fuel

fungi to biocides in diesel fuel

6.2

6.2 .1

SECTION II - Field studies of biocide effects

Effects of biocides on microbiological

contamination

6.2.2 Test on engine performance

6.2 .3

Corrosion test

6.3 Discussion

CHAPTER 7 SUMMARY

\section{APPENDICES}

3.1 Fungi isolated from different levels of tank 2 between 1982-1984

3.2 Fungi isolated from different levels of tank 3 
3.3 Fungi and bacteria isolated from the separating tank and wharf samples (1982-1984)

5.1 Partition coefficient of imazalil in the octanol-water system

6.1 The effects of the additives DEGME and Biobor JF on microbiological growth in diesel fuel at various levels of the tanks in the field trial at Devonport (April 1984 - August 1984).

6.2 The effects of the biocides DML-7 and Proxel AS on 183 microbiological growth in diesel fuel at various levels of the tanks in the field trial at Devonport (October 1984 - February 1985)

6.3 The effects of the biocides DML-7 and Proxel AS on microbiological growth in diesel fuel field trial (May 1985). Fuel sample replication study

6.4 The effects of the biocides benomyl and imazalil on microbiological growth in diesel fuel at various levels of the tanks in the field trial at Devonport (July 1985 - November 1985)

6.5 The effects of the biocides benomyl and imazalil on 189 microbiological growth in diesel fuel field trial (July 1985 - November 1985). Field sample replication study

6.7 Diesel engine additive trial graphs showing fuel usage, temperature versus time 
1.1 Ship fuel system with water compensated tanks.

1.2 Ship fuel system - steam turbine powered engine with non-water compensated tanks.

2.1 The fuel tanks and the fuel reticulation system.

2.2 Sectional view of the cliff storage tank and ventilation system.

$2.3 \mathrm{~A}$ The weighted sampling can.

B The zone sampler or 'thief'.

3.1 The occurrence of the predominant fungi isolated from 44 stored diesel fuel in relation to temperature and humidity (1982-1984).

3.2 Comparing the occurrence of the predominant fungi in the separating tank with that of tanks 2 and 3 (19821984).

3.3 The occurrence of the predominant fungi in the atmosphere and the storage tanks 2 and 3 (1982-1984).

4.1 Growth of Cladosporium resinae, Paecilomyces variotii and Penicillium corylophilum (ex diesel fuel) in Bushnell-Haas/diese1 fuel interface after six weeks.

4.2 Growth of fungal isolates on various aqueous/diesel fuel phases $(1: 1)$.

4.3 Growth of various types on aqueous/diesel fuel phases.

4.4 Growth of the various isolates on V-8 juice agar containing different concentrations of creosote. 
4.5 Light micrographs of conidiophore and conidia

a. Cladosporium resinae $f$. avellaneum ex diesel fuel.

b. Cladosporium resinae $f$ avellaneum ex jet fuel.

c. Cladosporium cladosporioides ex soil.

d. Paecilomyces variotii ex diesel fuel.

e. Penicillium corylophilum ex diesel fuel.

4.6 Scanning electron micrographs of conidiophores and conidia

a. Cladosporium resinae $f$ avellaneum ex diesel fuel.

b. Cladosporium resinae $f$. avellaneum ex jet fuel.

c. Cladosporium cladosporioides ex soil.

d. Paecilomyces variotii ex diesel fuel.

e. Penicillium corylophilum ex diesel fuel.

4.7 Transmission electron micrographs showing the conidia of Cladosporium resinae (ex diesel) prepared using different techniques.

4.8 Low power transmission electron micrographs of
a. Cladosporium resinae ex diesel fuel.
b. Cladosporium resinae ex jet fuel.
c. Cladosporium cladosporioides ex soil.

4.9 a Transmission electron micrograph showing the cell wall structures of Cladosporium resinae.

b\&c Electron micrographs of plasma membrane of Cladosporium resinae.

4.10a\&b Electron micrograph of the intracellular conidial structure of Cladosporium resinae.

4.11 a. Conidia of Cladosporium resinae with the large electron-dense body.

b. Electron micrograph of the ramoconidia of Cladosporium resinae. 
4.12 a. Microbody adjacent to the septum.

b. Woronin body and electron-dense bodies adjacent to the septum.

5.1 Apparatus for agar slide culture technique.

5.2 Sectional view of the small steel tank showing the 101 sampling points.

5.3 The cylinder head of the Enfield VS1 engine.

6.1 The growth of treated and untreated fungal colonies in the poison plate test.

6.2 Piston showing carbon deposit thickness measurement positions. 


\section{LIST OF TABLES}

2.1 Growth studies of single species in various media.

2.2 Isolates of fungi scanned for their tolerance to creosote.

2.3 The effects of road transportation on spore viability.

2.4 Summary of fixation procedures for transmission

electron microscopy (TEM) studies.

3.1 Fungi isolated and identified from New Zealand stored diese1 fue1 (1982-1984).

3.2 Mean number of colony forming units (CFU) and frequency of isolation of fungi from diesel fuel stored in tanks 2 and 3 over a twenty-four month sampling period.

3.3 Mean number of colony forming units (CFU) and frequency of isolation of fungi from the separating tank, Shell tank, tank cleaning vessel and wharf sampling point.

3.4 Incidence and levels of bacteria found in diesel fuel at different testing dates.

3.5 Numbers of bacterial colonies per litre obtained from tanks 2 and 3 and the separating tank (1982-1984).

3.6 Comparison of two media and various modified membrane filter techniques for two separate fuel samples.

3.7 Effect of time of testing of samples and container type 52 on recovery of Cladosporium resinae. 
3.8 Fungi isolated from the atmosphere by the KramerCollins spore sampler during survey period 1983-84, growing on V-8 juice agar containing $0.1 \%$ creosote.

4.1A Growth of principal microbial contaminants in the laboratory on various aqueous/diesel fuel phases.

4.1B Viability of fungi/spores isolated after six weeks and 67 grown on V-8 juice agar.

4.2 Growth and survival of Penicillium spp., Cladosporium resinae and Paecilomyces variotii on Bushnell-Haas plus diesel or jet fuel.

4.3 Initial growth of Cladosporium resinae followed by growth of Penicillium corylophilum and Paecilomyces variotii on the same batch of various aqueous/diesel fuel phases.

4.4A Growth of Candida albicans, Cladosporium resinae, Penicillium corylophilum, Penicillium expansum, Paecilomyces variotii as single and various mixed cultures on aqueous and aqueous/diesel fuel phases.

4.4B Viability of fungi in samples from six weeks old cultures.

4.5 The effects of growing selected fungi which support the growth of other fungi.

7


6.4 Growth of various Cladosporium resinae isolates on MEA as affected by biocides (Poison plate test).

6.5 Determination of biocidal activity of benomyl (B) and imazalil (I) alone or in mixture at varying concentrations in poison plates on Paecilomyces variotii (PV) and Cladosporium resinae (CR) after eight days.

6.6 Spore germination and growth in Bushnell-Haas/diesel fuel phases.

6.7 Viable microbial contamination during biocide treatment of large volumes of diesel fuel.

6.8 Colony forming units surviving after exposure to biocides intermittently.

6.9 The effect of the additives DEGME and Biobor JF on microbiological growth in diesel fuel field trials at Devonport (April 1984 - August 1984).

6.10 The partition coefficient of the compounds studied in the field and the water content in each of the treated tanks.

6.11 The effects of the biocides DML-7 and Proxel AS on microbiological growth in diesel fuel field trials at Devonport (October 1984 - April 1985).

6.12 The effects of the biocides benomyl and imazalil on microbiological growth in diesel fuel field trials at Devonport (July 1985 - November 1985). 


\subsection{Studies on microbiological contamination of hydrocarbon fuels.}

All heterotrophic micro-organisms, whether bacteria or fungi, have similar general requirements for growth. They require a source of organic carbon to supply both carbon skeletons for the formation of new cell constituents and to provide the necessary energy following partial or complete oxidation. Such organisms have been called chemoorganotrophs (Hawker and Linton, 1979). Hydrocarbon fuels contain between $80 \%$ and $89 \%$ carbon (King and McKenzie, 1977) and thus should be able to provide an excellent source of carbon energy. However they vary in their susceptibility to microbial degradation.

Certain organic materials which are now quite common environmental contaminants were not always so common, and the hydrocarbons which comprise the various types of modern liquid fuels are prime examples. In recent years due to the rapid development in industry, transportation and naval activity large quantities of fuel are stored for long periods providing a man-made environment where micro-organisms can thrive. Many other man-made organic molecules such as plastics and other synthetic materials widely used by man provide even more recent additions to the environment and may be capable of forming a substrate for microbial growth. The ability of micro-organisms to metabolise hydrocarbons was demonstrated long ago by Miyoshi (1895). More recently it has been shown that a range of microbial species have the capacity to break down various hydrocarbons (ZoBel1, 1946; Beerstecher, 1954; Foster, 1962).

Aliphatic and long chain hydrocarbons appear to be more susceptible to microbial attack than aromatic and short chain hydrocarbons. Molecules containing from 10 to 18 carbons are attacked more frequently and rapidly, and they support abundant microbial growth (Lukins, 1962). The reason fuel containing aromatic hydrocarbons is less readily attacked, may perhaps be due to toxicity or the requirement for more specialised enzymes to degrade such compounds (ZoBe11, 1950). 
Other nutrients namely nitrogen, phosphorus, potassium and trace elements are less abundant in fuel, although their levels may be supplemented by fuel additives, rubber, paints, human waste and seawater (Hill and Hughes, 1969).

It is therefore not surprising to find micro-organisms associated with aviation fuels (gasoline and kerosene in both subsonic and supersonic aircraft and in storage tanks), diesel fuel (middle distillate fuel) in storage, in road and rail vehicles, diesel generators, central heating fuel and diesel fuel onboard ships, particularly where water displacement is used in order to maintain a consistent 'trim'. Green et a1. (1967) reported that rocket propellants were also susceptible to microbial attack. The hydrocarbons are predominantly aliphatic but in some fuels, a high content of aromatic hydrocarbon may be present, for example some jet fuel contains $25 \%$ aromatics. The additives which may be present in the aviation fuels include anti-oxidants, metal deactivators, corrosion inhibitors, antistatics and anti-icing agents. The term 'diesel fuel' covers a wide range of differing hydrocarbon blends, some of which may also contain cetane number improvers and corrosion inhibitors (Genner and Hill, 1981). The navy uses a special type of marine diesel called 'dieso'.

All organisms including hydrocarbon utilizing organisms depend upon a certain amount of water in various forms. Growth of microorganisms appears to occur in the water phase which is nearly always found in commercial hydrocarbons. For example hydrocarbon fuels will absorb water from the atmosphere to the extent of $100 \mathrm{ppm}$ and this tends to separate out to generate the free water phase when subjected to temperature or pressure fluctuations. If hydrocarbon fuels are completely dry, it would appear that growth cannot occur but some contaminants may survive. Survival of cells can vary considerably (Sharpley, 1966). Bacteria may survive for only a few hours while resistant stages such as the conidia of Cladosporium resinae (anamorph of Amorphotheca resinae) are known to survive for prolonged periods (Hil1, Evans and Davies, 1967). Survival of microbial spores in dry hydrocarbons also appears to vary depending on temperature (Sharpley, 1966). 


\subsubsection{Early reports of microbial attack}

The earliest published reports on bacterial attack of fuels and oils were in the 1930's. Tausson and Aleshina (1932) reported attack on fuel by anaerobic sulphate-reducing bacteria. Thaysen (1939) investigated the explosion of a kerosene storage tank and found that growth of sulphate-reducing bacteria was responsible due to evolution of gas during their growth. The first report of yeasts assimilating hydrocarbons was by Tausson (1939). He reported that species of Debaryomyces, Endomyces, Hansenula, Torulopsis and Monilia were capable of growth on hydrocarbons.

Bushnell and Haas (1941) studied the micro-organisms present in the water bottoms of aircraft storage tanks, and found that many of the bacteria present were capable of hydrocarbon oxidation and could utilize gasoline and kerosene. Allen (1945) also studied the effects of bacterial growth on gasoline in storage tanks. He concluded that a major factor in the escalation of this problem was due to the increased storage at higher temperatures and this had increased the incidence of microbial growth in the fuel tank water bottoms.

Evidence that corrosion could result from microbial growth on hydrocarbons was presented by Ganser (1940). He reported that sulphate-reducing bacteria were responsible for corrosion in pipelines. In the 1960's aviation gasoline and jet fuel on aircraft carriers and amphibious assault ships became corrosive from fuel soluble sulfide generated from reduction of sulphate by anaerobic bacteria in the seawater stored in the bottom of storage tanks. (Kelley, 1967; Klemme, 1968).

The first reports of problems arising from bacterial attack of jet aircraft kerosene were published in the late 1950s. Bakanauskas (1958) found that fuel-filter clogging in aircraft was due to the bacterial slime formed at the fuel/water interface in the tanks of the aircraft and that fungi were not present. However, the mechanical strength of these bacterial slimes was subsequently thought to be insufficient to cause filter blockage implying that fungi were present. 
De Gray and Killian (1960) reported that bacterial slimes occurring in fuel-storage tanks could increase corrosion of tanks and pipelines, due to the production of low molecular-weight organic acids which lowered the $\mathrm{pH}$ value of the aqueous phase. These acids could also react with mineral salts in solution to produce more corrosive acids.

The first report of fungal activity in jet fuel systems was by Klemme and Leonard (1960) who found that Hormodendrum resinae (later known as Cladosporium resinae) was the predominant fungus present. Hazzard (1961) reported that fuel tank corrosion in Lockheed aircraft was associated with fungal contamination of the fuel. He found that fungal growth was present in a high proportion of samples taken from storage tanks, aircraft tanks, tankers, refuelling vehicles and pipelines. Thereafter careful, extensive and comprehensive studies were carried out by many researches on the history of the problem of microbial contamination of aviation fuel and the development of control measures (Stormont, 1961; Parbery, 1971; Sheridan, Nelson and Tan, 1971; and Genner and Hil1, 1981).

Cladosporium resinae possibly appeared in diesel fuel at the same time as in the aviation fuel, but because the fuel was used in less sophisticated diesel engines it probably passed unnoticed until its presence in jet fuel caused concern. A comprehensive survey by Liggett (1976) of marine, rail and road diesel fuels, tractor vapourizing oil, paraffin, gas oils and central-heating fuels gave a clear indication of the widespread nature of the microbial contamination. This survey also reported associated system malfunctions, particularly filter plugging and lowering of interfacial tension between fuel and water. Hostetler and Powers (1963) have demonstrated that injector failure in diesel locomotives can be attributed to contamination of the fuel and they quote figures indicating that biocides minimize formation of 'gum' sediments and filter plugging in heating oils, rail and road vehicle fuels.

Cladosporium resinae was found to be present in 'dieso' and moreover this fungus was reported to constitute the bulk of the 'biomass' (Houghton and Gage, 1979; Neihof, 1980; Bruce, 1982; Arnold, 1983). 
Some bacteria and fungi isolated from hydrocarbons, do not survive in fuel for as long as the fuel-degrading organisms. This suggests that these are chance contaminants introduced through inadequate fuel sampling techniques or through recent fuel system contamination (Cooney and Kula, 1970).

The micro-organisms found in fuel can originate from a variety of sources. Bacteria and fungi may find their way from air, water and soil into storage tanks, oil-fired furnaces, diesel locomotives, trucks, aircraft and ground storage tanks. Co resinae, the common contaminant of kerosene and diesel, is known to be widespread in soils and as airborne spores in Australia, British Isles and Europe (Parbery, 1969) and New Zealand (Sheridan, 1972). The ubiquitous presence of $C$. resinae suggests that it will eventually find its way into fuel tanks but such contamination may be harmless unless water is present to provide the ideal habitat for growth (Sharpley, 1966).

As this study is focussed on the microbiological contamination of diesel fuel used mainly by frigates, it is appropriate to discuss the type of tanks used in warships and how microbiological contamination could affect the fuel system. Warship fuel systems provide basically two different microbial environments.

These are as follows:

(i) Seawater-displaced fuel tanks (water compensated tanks) may contain a high ratio of water to fuel (refer Figure 1.1) and offer a wide range of nutrients, in addition to those already in the fuel. The nature and quantity of these supplementary nutrients will depend on the type of water used to fill the header tanks, which may range from highly saline deep-seawater to estuarine water. Factors such as sewage content and trace metal contamination of the water may affect the extent of colonization (Neihof and May, 1983).

(ii) Undisplaced fuel tanks (non-water compensated tanks) contain a low ratio of water to fuel and generally less particulate organic matter. Figure 1.2 illustrates such a system. Nutrient levels will be determined by the previous history of the fuel and subsequent factors such as deterioration of tank linings and seals. 

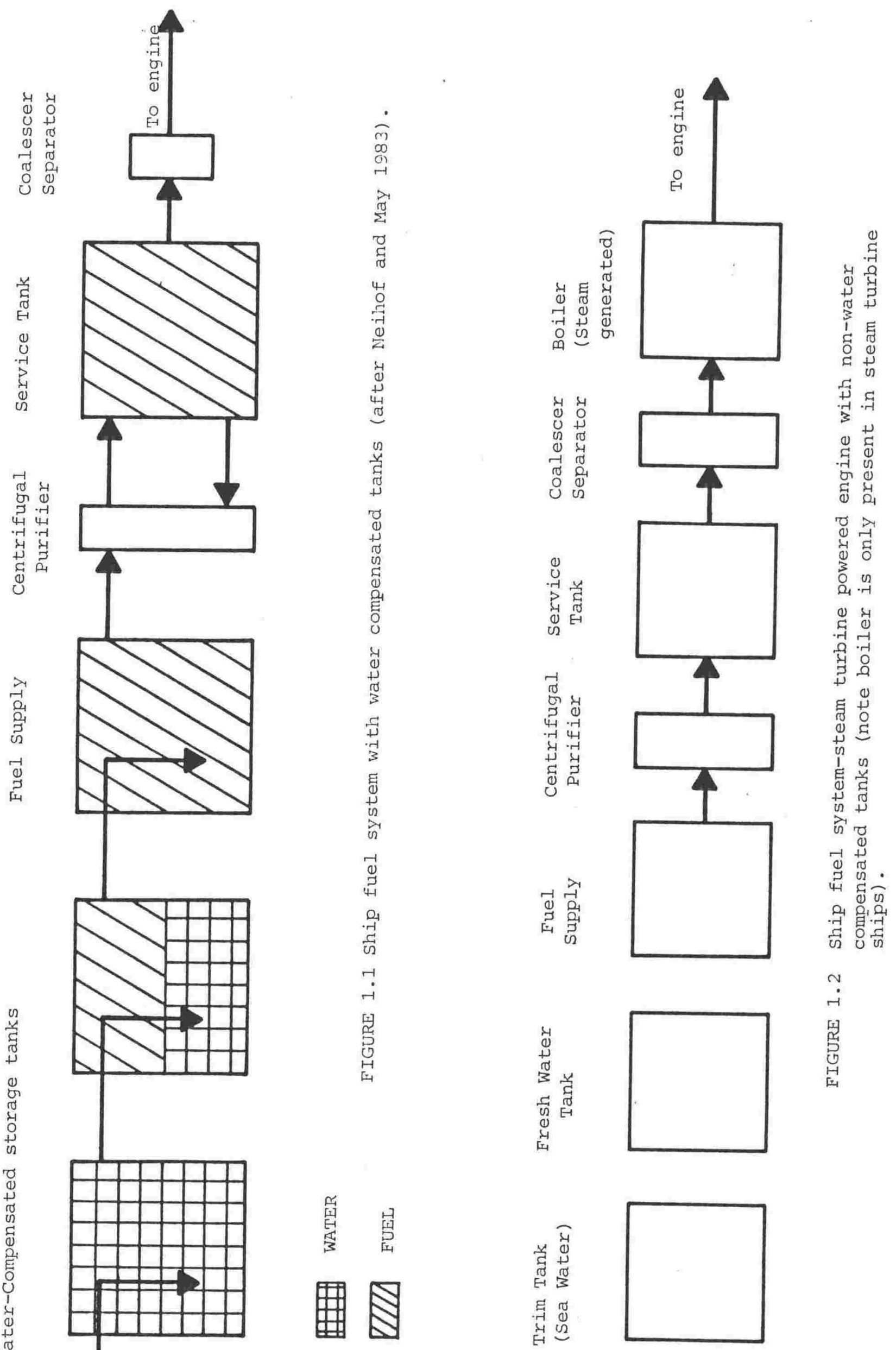
Both the above systems may use the coalescer to effect separation of entrained water. In the coalescer elements a continuous flow of fuel and water passes over ensnared organisms, supplying their nutrient requirements and removing their metabolic products. Degradation of the cotton, used in the construction of the coalescer elements, may supply alternative carbon sources. During the fuel's journey through the system, the concentration of some nutrients decreases, while levels of metabolic and cell lysis products may increase (Turner, Eaton and Jones, 1983).

The sea-water displaced system of both steam and gas turbine powered ships was proved to be vulnerable to fuel system blockages resulting from microbial contamination of fuel. Prior to delivery to engines the solid matter and water content of the 'dieso' could be reduced by filtration and water separation techniques. High levels of fuel sludge result in premature failure of filters and water-separator systems and may cause acute fuel starvation (Turner, Eaton and Jones, 1983). The fuel temperature reflects the external sea temperature and hence can be expected to vary from $4^{\circ} \mathrm{C}$ to $25^{\circ} \mathrm{C}$, which supports microbial growth.

According to Genner and Hill (1981), microbial growth in fuels can result in several types of problems which can be grouped into two major classes, whether they are caused by metabolism of organisms or by the physical presence of organisms.

Both corrosive substances and gas are known to be produced during microbial metabolism. For example it has been found that four carboxylic acids were produced by $C$. resinae (Rivers, 1973; McKenzie, Akbar and Miller, 1976) and they were identified as dodecanoic, acetic, glycollic and glyoxylic acids (Siporin and Cooney, 1975). Studies of the corrosive effect of $C$. resinae have centred on aluminium and its alloy: pitting, surface exfoliation, blistering of the oxide layer, and intergranular corrosion have commonly been reported, as well as general weight loss (Hedrick et al., 1965; Parbery, 1968; Rivers 1973; A1-Haidary, 1977; Hansen, Tighe-Ford and George, 1981). 
The acid metabolites increased corrosion by lowering the pitting potential of aluminium and aluminium-copper alloy (de Male, Salvarezza and Videla, 1979; de Meybaum and de Schiappare11i, 1980; de Schiapparelli and de Meybaum, 1980). Clearly, meshed fungal mycelium would lead to physical blockages of pipeline filters and drain holes and malfunction of coalescers and fuel gauge probes.

The technical innovation of using steam and gas turbines as power units for naval vessels has been accompanied by predictable microbial problems. Diesel fuelled gas-turbine powered engines, most common in American and British frigates, use water displaced tanks which are likely to be affected by the consequences of microbial growth. Here seawater displaces fuel as it is used to eliminate vapour space in the tanks. The Royal N.Z. Navy ships have steam turbine-powered engines using both displaced and undisplaced fuel tanks systems.

In the gas-turbine powered engines, the diesel is ignited to produce gas which drives the turbine. It is operated on a similar principle to a jet aircraft engine. In the steam-turbine powered engine, the diesel is injected into the boiler. Water is constantly circulated through tubes in the boiler, where the diesel is fired to convert the water into steam. Thus the steam drives the turbine. It works on the same principle as the steam driven turbine in a thermal power station.

Ships using water displacement systems have encountered occasional difficulties from rapid accumulation of particulate matter in centrifugal purifiers and coalescer filters (Neihof and May, 1982). A centrifugal purifier is designed to remove most of the particulate matter and entrained water before the fuel enters the service tank. There are no centrifugal purifiers in the fuel system in N.Z. frigates (Defence Scientific Establishment (DSE) per. comm•). Coalescer filters remove residual water before the fuel goes to the engine. The ground storage tanks used by the New Zealand Navy acquire water bottoms by rain-water seepage and condensation of water carried in the fuel. The microbial growth occurs at the interface of this aqueous phase. 


\subsubsection{Morphology of Cladosporium resinae.}

Cladosporium resinae (Lindau) de Vries is the anamorph of the 'kerosene fungus' Amorphotheca resinae Parbery. It has recently been renamed Hormoconis resinae (Lindau) Von Arx and de Vries (1973). The reason for transferring the 'kerosene fungus' to the genus Hormoconis is based on the lack of prominent basal scars on the conidia, diagnostic of the genus Cladosporium. Since $C$. resinae is widely used and accepted, this name is used throughout this study. Four forms have been identified. Two forms have morphologically distinct reproductive structures $-f$. avellaneum and $f$. resinae. An albino may possess either morphology $-f$. albidum and there is a sterile form f. sterile (de Vries, 1952; Parbery, 1969; Sheridan, Tan and Nelson, 1972). Intermediates and variants of each form exist (Tan, 1972). This variability, together with variation in the ability to grow on petroleum products such as aviation kerosene, diesel and in other substrates including creosoted wood (Christensen et al., 1942; Marsden, 1954), wood treated with copper-chrome or arsenic preservatives (Nilsson and Henningsson, 1977), Coniferous resin and asphalt pavements have stimulated research interest in this fungus. These substances are normally considered to be fungistatic or fungicidal, yet $C$. resinae can utilize these as a source of carbon. Studies of anatomy and ultrastructure using both the scanning and electron microscope have been attempted to explain the properties of this most unusual fungus (Tan, 1972; Sheridan and Troughton, 1973; Soteros, 1973; Cooney, Siporin and Smucker, 1980; Smucker and Cooney, 1981; Smucker and Cooney, 1983).

\subsection{Control of microbiological contamination in hydrocarbon fuel.}

Solutions to the problem of microbial growth in diesel fuel systems have been sought by various groups (Landsdown, 1965; Edmonds, 1966; Hill et al., 1967; Hendey et al., 1971; Siporin and Cooney, 1975; Upsher, 1976; Houghton and Gage, 1979; Turner, 1981; Wycislik and Allsopp, 1983). The general conclusion from these studies is that there are several ways to prevent microbiological growth in fuel systems: good housekeeping, the addition of biocides and the use of tank linings. 
Good housekeeping and biocides can be used to minimize both contamination and growth, while tank linings can minimize corrosion should contamination occur. The control of microbial contamination by application of heat directly to the fuel on ships or in land storage depots has been considered (Upsher, 1976; Houghton and Gage, 1979; Wycislik and Allsopp, 1983).

There have been reports of a novel type of control in New Zealand, called the 'hydromag' (news report Anon, 1986). This device is described as a small inexpensive filter containing circular ceramic magnetic disc plates through which the fuel passes on its way to conventional filters. These are now installed in trial Auckland Regional Authority buses (ARA pers. comm.). The preliminary results were reported to be favourable but inconclusive. The use of 'hydromag' may not be practical in large volume storage tanks at ground installations or in the frigates. The corrosion problems associated with microbial contaminants during storage, and in the fuel lines may not be eliminated by the use of this device.

Good housekeeping primarily involves removal of free water from ground storage tanks and onboard ship tanks thereby minimizing the likelihood of microbial growths becoming established. In underground fuel installations such as those of the N.Z. Navy at Devonport, free water cannot be entirely removed because of the poor design of the storage tanks system. Inevitably, water is present and the use of biocides must be considered. In Australia, the tanks are designed to have conical bottoms to drain water readily (Sheridan per. comm.). Corrosion is not a problem in the underground storage tanks because the walls are made of concrete. It could be a problem, however, further downstream particularly in tank ships. The fungus metabolizes fuel by beta-oxidation to produce carboxylic acids (Cooney and Proby, 1971; Walker and Cooney, 1973). Large volume and rapid turnover prevents any significant change in composition of fuel (Hill, 1978); but the acid production can cause a drop in $\mathrm{pH}$ of water bottom and this can lead to increased corrosion of metal tanks. Good fuel maintenance is not however the complete solution, it appears that the only practical method for control of $C$. resinae and other predominant fungi is use of appropriate biocides. 
The effect of addition of biocides to jet fuel has been widely studied (Klemme and Leonard, 1960; Hitzman, Shotton and Alquist, 1963; Hendey, 1964; Hedrick and Carro11, 1966; E1phick and Hunter, 1968; Rogers and Kaplan, 1968; Rabotnova et a1., 1970; Park, 1973; Miller, Mohan and Strickland, 1975; Klemme and Neihof, 1976; Neihof and Bailey, 1978). Few studies have been made on diesel fuel (Stormont, 1962; Hostetler and Powers, 1963; Smith and Crook, 1980; Smith and Crook, 1983). Little work has been done on the effects of biocides on engine performance and the components of the combustion chamber.

Singer (1976) has described a method for recognizing biocidal activity in fuel. Elphick and Hunter (1968) and Rogers and Kaplan (1968) have described experiments which differentiated between biocidal and biostatic properties; but these were only qualitative. Smith and Crook (1980) described experiments which gave quantitative results on the effects of biocides in contaminated fuel.

According to Miller, Mohan and Strickland (1975) studies to identify biocides for the inhibition of growth of micro-organisms in 'dieso'-water systems have indicated that in addition to in vitro broad spectrum biocidal activity, such compounds must exhibit high solubility in the fuel coupled with an effective partitioning between the fuel and the water phase where the growth of micro-organisms occur. The water to fuel ratio can markedly influence the effectiveness of the biocide, a 1:40 ratio being considered ideal for micro-organism growth (Bennett, 1974). The narrower the water to fuel ratio, the smaller is the concentration of biocide required to inhibit the fungal growth.

\subsubsection{Criteria for the choice of fuel biocides}

The choice of a fuel biocide is restricted due to stringent specifications enforced by engine manufacturers. According to Hill (1982) the desirable properties of a biocide are as follows. It should:

- be combustible, with no residual ash

- not interfere with fuel properties or the combustion process, or any other aspects of engine performance 
- be soluble (or very miscible) in fuel but preferentially soluble in water

- present no health hazard during handling or combustion

- not be corrosive

- preferably achieve a total 'kill' of micro-organisms at minimum dose.

To this list could be added that it must be environmentally acceptable especially where seawater is used for displacement. No such biocide has been developed for commercial use. However a few biocides have achieved market penetration, although deficient in some of the listed characteristics. These are Biobor J.F., EGME and DEGME widely used in jet fuel.

Numerous reports in the literature indicate the effectiveness of EGME (Hitzman, 1964; London, Finefrock and Rillian, 1964) and organoboron compounds in jet fuel (De Gray and Fitzgibbons, 1966; Rogers and Kaplan, 1968; Elphick and Hunter, 1968). Elphick and Hunter (1968) focussed on the possible effectiveness of these materials when used as intermittent treatments for jet fuel systems while the others referred to the exposure of micro-organisms to biocides as continuous additives to jet fuel.

It is important to consider the intermittent use of the available chemicals, because factors may arise which militate against the use of biocides as continuous additives to fuel. There may be physical or economic reasons associated with the incorporation of biocide in fuel at a number of different ports where a ship may be required to refuel. A ship may be treated with biocide-containing fuel, and allowed to stand thus fuelled during routine maintenance periods. At the end of these periods this fuel may either be drained off and the ship refuelled with untreated fuel, or the biocidecontaining fuel may be used during the next sail, subsequent refuelling being with untreated fuel. Alternatively, or in addition to this procedure, a ship may receive biocide-containing fuel at more or less regular intervals during service, e.g. at one or more of its regular refuelling points. 
Biobor JF (U.S. Borax \& Chemical Corporation), a mixture of two organoboron compounds, was recommended for use at $270 \mathrm{ppm}$ for disinfection and $136 \mathrm{ppm}$ for continuous dosage in jet fuel. It has been found, however that Biobor JF when used at more than ten times the recommended dose rate in jet fuel still allowed $C$. resinae to remain viable for up to six weeks (Berner and Ahearn, 1977) and a dose rate of 5000 ppm was necessary to kill vegetative hyphae within two days (Singer, 1976). The use of Biobor JF has been criticized by Klemme and Neihof (1976) who found that 20,000 ppm was needed in the water phase to control sulphate-reducing bacteria. Moreover, certain engine manufacturers currently discourage the use of organoboron on a regular basis in fuel because it leaves an ash on combustion and takes several days to be completely effective biologically (Genner and Hill, 1981; Hil1, 1982).

Ethylene glycol monomethyl ether (EGME) is used mainly in military aircraft as an anti-icing additive in the fuel to prevent ice formation from entrained water. It was reported by Hitzman et al. (1963) and Rogers and Kaplan (1968) to be effective in controlling microbiological contamination in kerosene type fuels. EGME at 1500 ppm $(0.15 \%)$ is used to provide both anti-icing and antimicrobial activity. The major deficiency, however, is that at low concentrations it is readily metabolised by micro-organisms and hence, if used intermittently, there is a real risk that growth will be promoted (Hill, 1982). EGME affects the flash point of the fuel and there are moves to replace it with a related compound, DEGME (di-ethylene glycol monomethyl ether). This was reported to be a suitable replacement as it was effective as a biocide at 10,000 20,000 ppm (1-2\%) in aqueous phase compared with $100,000-170,000$ ppm (10-17\%) for EGME (Neihof and Bailey, 1978). The dose rates of both biocides are relatively high and neither was likely to be very effective when the ratio of water to fuel was greater than 1:400 (Hill, 1982). Since 1983 DEGME has been used widely in the Australian Navy (Sheridan pers. comm.). 
There are reports (Genner and Hill, 1981; Hill, 1982) of attempts to prevent the emergence of resistant organisms by using mixtures of biocides at different concentration. Walters (1971) suggested that constant concentration treatment with biocides may cause difficulties under practical condition through the appearance of resistant microbiological mutants.

Kathon 886 (active ingredients are 5-chloro-2-methy1-4isothiazolin-3-one and 2-methy1-4-isothiazolin-3-one) was found to be biologically active in fuel when used in combination with EGME at the very low concentration of about 1 ppm (Thomas and Hil1, 1977). Benomyl and imazalil are agricultural fungicides and the former was found to be effective in suppressing growth of $C$. resinae at a concentration of $1 \mathrm{ppm}(\mathrm{W} / \mathrm{U})$ in a 1:1 'dieso' fuel/water ratio (Smith and Crook, 1983). Imazalil has been suggested as a potential biocide for diesel fuel (Janssen Pharmaceutica Ltd. pers. comm.) but no published material is available.

Biocides may present some hazard to ships' personnel and can have unfavourable environmental effects when they are off-loaded (Neihof et a1., 1979). Recently Neihof and May (1983) discouraged the routine use of a biocide in ships operated on diesel fuel with a water compensated system. It has been observed that fuel contamination has originated before the ships leave the shipyard because of the introduction of particulate matter in silt laden, polluted water into the tanks (Neihof and May, 1982). This imposed a burden on the centrifugal purifiers and provided a favourable environment for subsequent microbial growth. Most of the particulate matter would appear to be the fuel insolubles formed from inherently unstable fuels and an array of particles introduced with the ballast water. These workers have made recommendations for minimizing these sources of contamination.

Anti-microbial activity depends upon effective contact between the chemical and the micro-organism and the mechanism involves disruptive interaction with a biochemical or physical component of the organism which is essential to its structure or metabolism (Kostenbauder, 1977; Albert, 1979). 
The target may be a single enzyme, a cell membrane, a more generalised aspect of the cell or a combination of these and the nature of the action is dependent on the organism and the environment in which the interaction occurs as well as on the anti-microbial agent. For example quaternary ammonium agents are known to be surface active agents which may influence the cytoplasmic membrane and thereby upset the osmotic balance and permeability that are essential to the life of the cell. In fuel, quaternary ammonium compounds appeared to be particularly suitable, some resulting in sterilization after two hours exposure, and also they had good cleaning properties (Genner and Hill, 1981). However, they may lower the interfacial tension between fuel and water and become non-persistent and lose their activity progressively in emulsions, often within a few days and particularly above ambient temperature (Russell, 1982). Benomyl is known to interfere with mitosis by disrupting the normal assembly of the mitotic spindle (Davidse, 1973; Hammerschlag and Sisler, 1973). In aqueous solution, benomyl hydrolyses rapidly to form two compounds, carbendazim and butyl isocyanate, both of which show antifungal properties (Clemons and Sisler, 1969; Hammerschlag and Sisler, 1973; Chiba and Doornbos, 1974).

In New Zealand, major problems with diesel fuel blockages and fuel gauge failures have occurred in navy ships, in the 'Bounty' replica (pers. comm.), and in Auckland Regional Authority buses (News report-Anon, 1981). Little work has been carried out to establish the main cause of these problems. In the last decade the Royal N.Z. Navy has encountered continuous problems in frigates using water displacement tanks (DSE pers. comm.). The major problems involved fuel flow blockages and engine failures.

\subsection{Aims of Study.}

The present study was initiated under a contract with the New Zealand Defence Scientific Establishment. This concerned

(i) documentation of the nature, extent and possible sources of microbiological contamination of middle distillate fuel ('dieso') held in storage tanks at the RNZ naval base at Devonport, Auckland. 
(ii) study of possible methods of control of contamination using biocides. The effects of biocides on fuel effectiveness and engine corrosion were also considered.

Methods for detection of micro-organisms in diesel fuel held in the large underground storage tanks were evaluated. The most efficient method was used to document the micro-organisms present. Changes in the microflora were studied over a two year period. The influence of factors such as container type, transport, shaking and time on micro-organisms in samples after collection was investigated. Interactions between the predominant fungal contaminants were studied.

Commercially available and experimental biocides were added to diesel fuel and their effects in inhibiting growth of micro-organisms in the laboratory and in field tanks (25,500 litres fuel capacity) were studied. The aim was to find an effective biocide which did not have a deleterious effect on engine performance and life.

The morphology and ultrastructure of the major contaminants were also studied to extend previous observations of peculiarities that may explain their unusual metabolic capabilities. 


\section{PART 1}

\section{STUDIES ON MICROBIOLOGICAL CONTAMINATION OF DIESEL FUEL:}

\section{MATERIALS AND METHODS.}

\subsection{Sampling of storage tanks.}

\subsubsection{Tank description.}

Two cliff storage tanks containing diesel, at the Royal naval

base, Devonport, Auckland, were investigated for microbiological contamination. The horizontally placed, cylindrical tanks (numbers 2 and 3) are approximately $115 \mathrm{~m}$ long and $9 \mathrm{~m}$ deep. The fuel tanks and the fuel reticulation system are illustrated in figure 2.1. Underneath each cliff tank there are 'field tiles' consisting of a spaced series of short round, coarse ceramic pipes (300 mm long x 76 mm wide). These pipes are designed to absorb any diesel that soaks through the tank wall and also the ground water seepage. The liquids are channelled into a small sullage tank $(2.4 \mathrm{~m}$ high $\times 1.8 \mathrm{~m}$ diameter), one for each cliff tank. Within the bottom of each cliff tank, there is a sullage pipe that drains the water and some bottom fuel into the sullage tank. The sullage tank contents are pumped into the separating tank. Everyday as the fuel rises in the separating tank, it cascades over and is pumped directly into tank 3 while the water is pumped into the sea. There is no filter system in between tank 3 and the separating tank.

The tanks are adequately ventilated as shown in figure 2.2. Tank 3 was selected for fuel testing because it was the only tank where the fuel was constantly circulated. The fuel in tank 2 was frequently used by frigates and the fuel usage was higher than from tank 3. The fuel from all the cliff tanks flowed through the same pipeline to the wharf. 


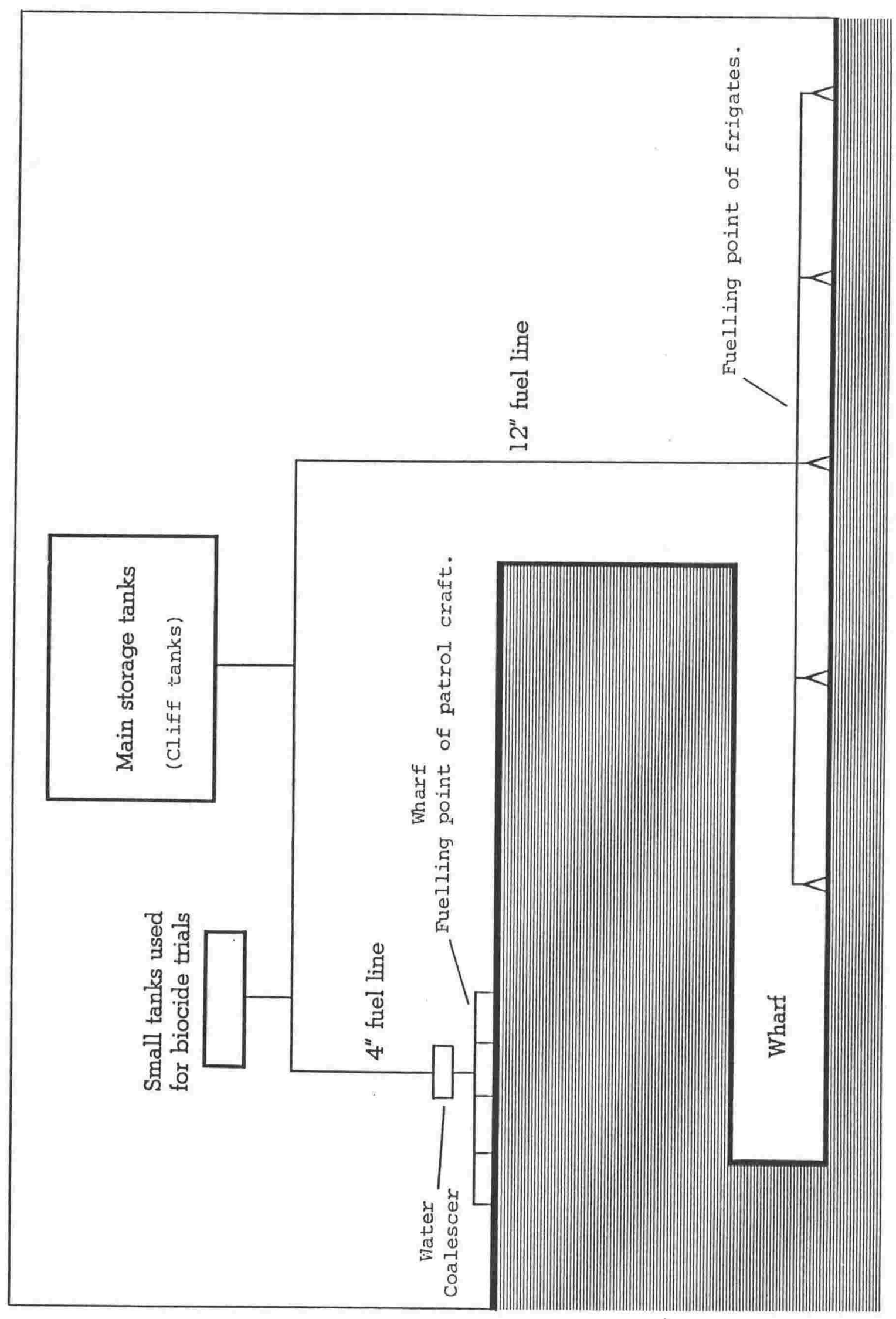

हٔ 


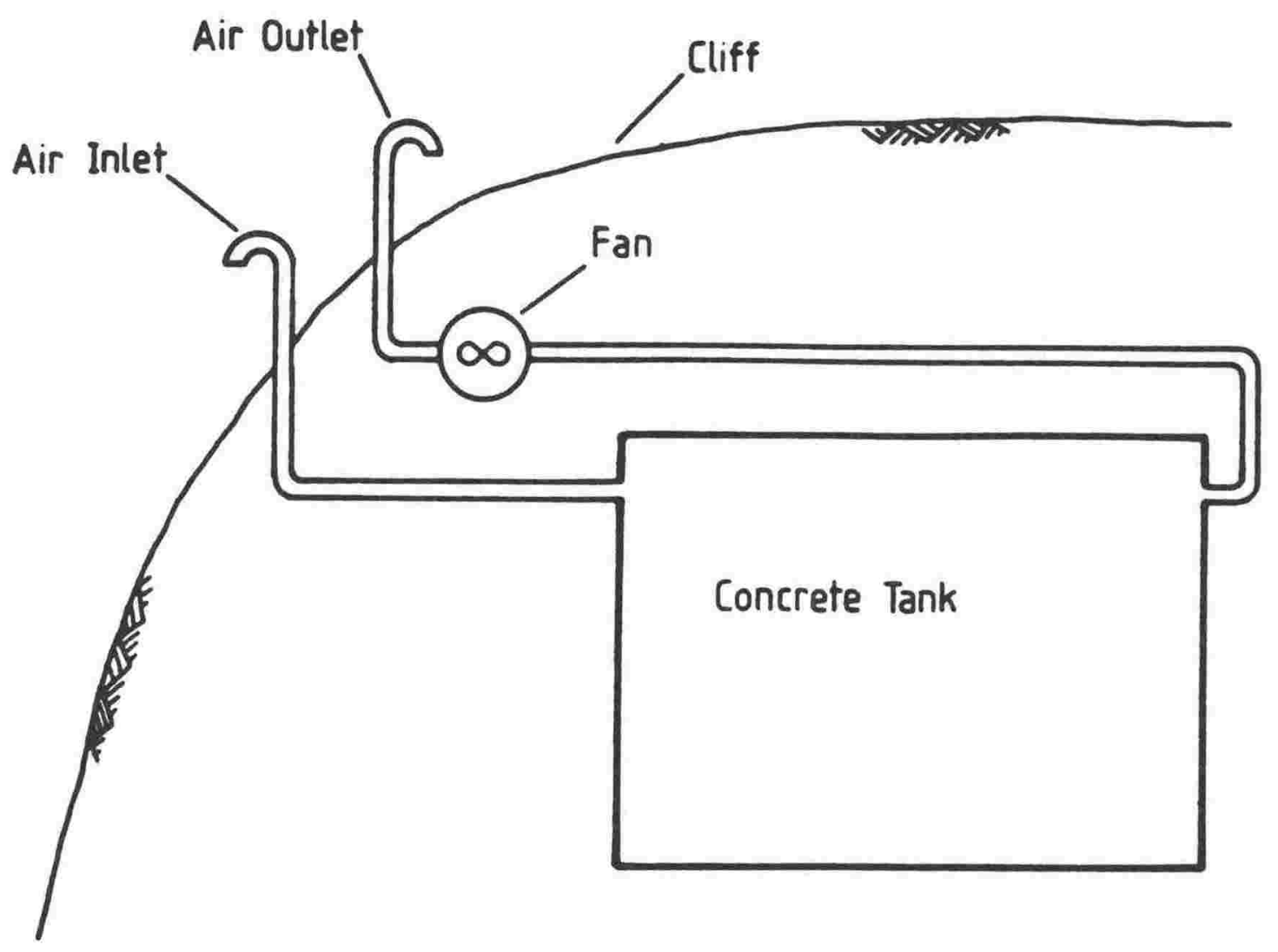

Figure 2.2 Sectional view of the cliff storage tank showing ventilation system. (tank not to scale)
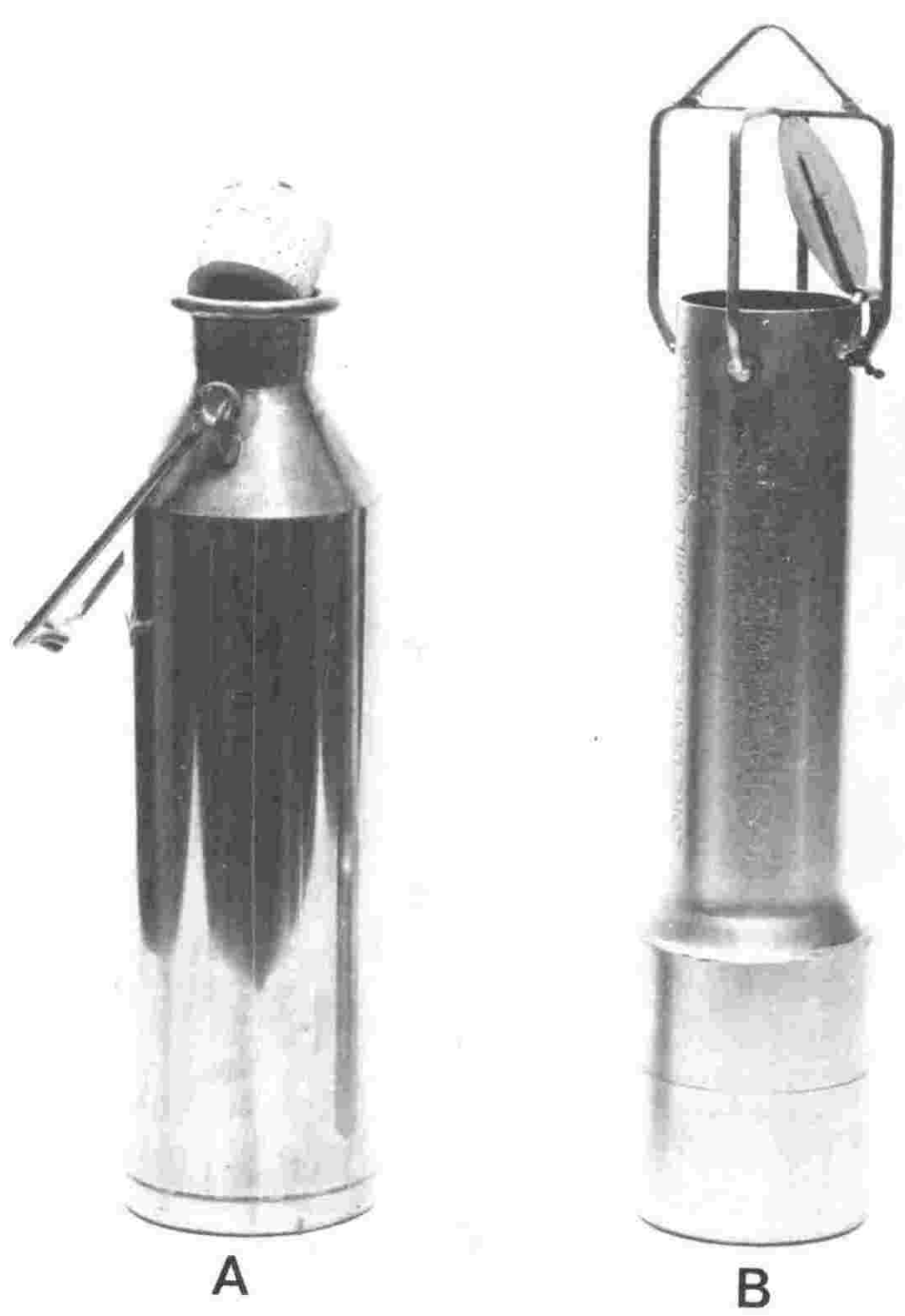


\subsubsection{Collection of samples}

A total of ninety three fuel samples from the top, middle, bottom and 'very bottom' of the two cliff storage tanks (tanks 2 and 3) were tested between May 1982 - December 1984.

In addition the following fuel samples were tested:

(i) Fuel and water sumps were collected separately from the separating tank to observe the contamination level in the fuel re-entering tank 3 .

(ii) Fuel from wharf samples was collected from the water coalescer (water separator) before the fuel is pumped into the patrol craft. This water coalescer (refer figure 2.1) is made of cotton and inside there is fine brass gauze. Later it became evident that it was important to test the fuel entering the patrol craft (after and not before the water coalescer) for biological contamination level. The fuel for the frigates is pumped directly from the cliff tank and not through the above water coalescer. Onboard frigates, before the fuel goes into the engine system, it is filtered through a coalescer which consists of glass fibre elements with a hydrophilic coating.

(iii) A single fuel sample was obtained from the Shell storage tank (situated directly on the opposite side of the Devonport wharf). Shell was the supplier of fuel to the base.

(iv) A single fuel sample from the tank cleaning vessel (TCV) was collected. TCV was used for cleaning and maintenance of the shipboard tanks. It also collects fuel from the Shell tanks and pumps the fuel into the cliff tank and occasionally pumps the fuel from the frigates to the cliff tanks.

These samples were important because they might establish the source of contamination in the ground installation tank. Due to management difficulties, it was impossible to obtain fuel samples from all of the above positions regularly. It was not possible to sample the fuel along the fuel line, since most of the pipes are buried underground. 


\subsubsection{Sampling methods}

Fuel from the top ( $0.6 \mathrm{~m}$ from the top of the tank ceiling), middle ( $2 \mathrm{~m}$ below the surface of the fuel), bottom ( $6 \mathrm{~m}$ below the surface of the fuel) of the storage tanks was sampled by a weighted sampling can (figure 2.3A). This is a brass bottle with a capacity of one litre. It fills with fuel after being lowered to the appropriate level where the stopper is removed by the captive rope.

The 'very bottom' of the tank was sampled by a zone sampler also sometimes called the 'thief' (figure 2.3B). This sampler allows the fuel to pass through as it descends through the fuel in the tank. This is made possible by the top and bottom valves opening due to the pressure of the fuel against the descending sampler. When the sampler contacts the very bottom of the tank, the valves close and the sample is retained as the sampler is retrieved to the surface. This fuel sample is thus from one $\mathrm{mm}$ from the very bottom of the tank to $300 \mathrm{~mm}$ to the top of the sampler.

The temperature of the fuel was recorded by dipping a thermometer into the fuel sample as soon as it was brought to the surface.

\subsubsection{Sampling pattern}

The fuel samples were immediately transferred into clean, sterile containers in such a manner as to minimize contamination from the environment and transported to Wellington, where the microbiological examination was carried out. Initially two types of containers: glass preserving jars and tin cans were used, to observe whether container type had any effect on the recovery of microorganism. It was found that glass bottles and tin cans were equally suitable. However the former were preferred because they do not corrode and are easier to clean and sterilize. For routine use the sampling cans and glass preserving jars were washed in detergent, rinsed with $95 \%$ alcohol and sterilized at $200^{\circ} \mathrm{C}$ in a hot air oven for three hours before collection of the diesel fuel.

Fungal and bacterial assays were carried out on the samples both immediately and twenty-four hours after sampling. 
Later this was extended to 4-5 days in order to detect any change in the composition and relative abundance of the microflora in the sample over time. As the time of testing of samples after collection did not appear to be critical, immediate testing in Auckland was discontinued. The fuel was always visually examined before subjecting it to microbiological procedures.

\subsection{Detection of micro-organisms in the fuel samples}

\subsubsection{Culture media}

$2 \%$ malt agar without peptone and dextrose and V-8 juice agar (Sheridan, Steel and Knox, 1971) were used for growth and isolation of fungi. Sterile Petri-dishes containing V-8 juice agar with creosote were used selectively to isolate $C$. resinae from the atmosphere (Sheridan and Nelson, 1971). Water agar was used on settle plates to trap air spora. Czapek-Dox agar (Pitt, 1979) was used as an identification medium for Penicillium spp. Nutrient and blood agar base (Collins and Lyne, 1976) were used to isolate bacteria. Selective media such as Starkey, Baar's agar (Sharpley, 1966), Bacto sulfate APl broth with and without sodium chloride (Hill, 1975) were used to isolate sulphur bacteria. MacConkey medium (Collins and Lyne, 1976) was used for the detection of $E$. coli. Bushnell-Haas mineral salt medium was prepared according to Parbery and Thistlewaite (1973) and used in conjunction with a selected hydrocarbon as carbon source. All the media were prepared by dissolving the agar before the constitutents were added and mixed. Sterilization was by autoclaving at $103 \mathrm{kPa}\left(121^{\circ} \mathrm{C}\right)$ for 15 mins, except in the following cases:

(i) for the blood agar, the sterile defibrinated horse blood was added to the autoclaved medium at $48^{\circ} \mathrm{C}$;

(ii) when creosote was used it was autoclaved and added aseptically to sterile molten media $\left(55^{\circ} \mathrm{C}\right)$ just before pouring the plates.

\subsubsection{Membrane filtration}

The fuel sample was shaken thoroughly before filtering through a membrane filter. The size of the fuel samples tested varied between $50 \mathrm{~cm}^{3}-200 \mathrm{~cm}^{3}$ depending upon the contamination level. 
Sterile cellulose acetate filters with a pore size $0.45 \mu \mathrm{m}, 47 \mathrm{~mm}$ diameter $(0.45 \mu \mathrm{m}$, filters, type HAW $0047 \mathrm{sl})$ and with a pore size $0.22 \mu \mathrm{m}(0.22 \mu \mathrm{m}$ filter GSWP 04700) were used to isolate fungi and bacteria respectively. Suction was applied by a mechanical vacuum pump for 30 seconds after the last drops of the sample had passed through the membrane. A trap was always used between the pump and the membrane, to prevent any fuel entering the pump. The pump was then disconnected and the membrane transferred, using sterile forceps, to the centre of a sterile Petri-dish. The selective medium (at $55^{\circ} \mathrm{C}$ ) was poured over the filter membrane and the Petri-dishes were incubated at $25^{\circ} \mathrm{C}$ in the dark for 5 days.

Whenever the samples exuded the strong 'rotten egg sme11' characteristic of sulphur bacteria, or if there was an increase in water at the tank water bottom, the water sumps and the 'very bottom' samples from the cliff tanks were tested for sulphur bacteria. The samples were filtered using $0.22 \mu \mathrm{m}$ filters and these were plated on sterile Petri-dishes. The selective medium (see culture media) was poured over filters. These plates were incubated in gaspak anaerobic jars at $35^{\circ} \mathrm{C}$ for 5 days.

On the 19th April 1983, it was suspected that sewage could have leaked into the tank. Thus the samples were tested for the presence of Escherichia coli ( $E$. coli). The water samples from the very bottom of tanks 2 and 3 and the separating tank were filtered using $0.22 \mu \mathrm{m}$ filters. The MacConkey medium was poured over the membranes and the plates were incubated at $37^{\circ} \mathrm{C}$ for 24 hours. The bacteria isolated from the water samples were tested for breakdown of lactose and the subsequent production of acid and gas at $44^{\circ} \mathrm{C}$, a test which is diagnostic for $E$. coli (Collins and Lyne, 1976).

Quantitative estimation of the level of contamination in the tanks was made by recording the number of colonies of each fungus on each plate and the number of samples in which a colony forming unit of a particular fungus occurred. Each discrete colony formed by a fungus on a culture plate was recorded as one colony forming unit (CFU). 
For each sample assayed five aliquots were filtered and the counts of $\mathrm{CFU}$ recorded are the mean number of colonies from these five filters for each fungus.

\subsubsection{Direct Observation}

Duplicate $100 \mathrm{~cm}^{3}$ of each sample were filtered separately using a $0.45 \mu \mathrm{m}$ filter. The filters were then transferred aseptically to sterile Petri-dishes. The spores were either stained with cotton blue or mounted in colourless lactophenol and examined under the phase contrast microscope immediately to record the range of spore types present.

\subsubsection{Pour plate method}

Duplicate samples of $5 \mathrm{~cm}^{3}$ from water sumps, from the separating tank and sometimes water from the 'very bottom' sample from tanks 2 and 3 were aseptically pipetted directly into sterile Petridishes. Molten selective culture media at $45^{\circ} \mathrm{C}$ was poured over the sample and mixed by rotating the dish clockwise and anticlockwise to disperse the organisms. After incubation for 5 days the colonies were identified and counted.

\subsubsection{Spread plates}

Samples of fuel or water bottom were vigorously shaken and duplicate samples of $5 \mathrm{~cm}^{3}$ were pipetted onto a selective agar medium and a sterile bent glass rod was used to spread the sample over the entire surface of the plate. To observe the growth of anaerobic sulphur bacteria, the plates were incubated in an anaerobic gaspak jar at $35^{\circ} \mathrm{C}$ for 5 days.

\subsection{Detection of micro-organisms in air and soil}

Initially the air spora in the immediate vicinity of the storage tanks was examined by the settle plate method. Water agar plates were exposed in duplicate inside and outside the area surrounding the tanks at different times during the day. In the laboratory, V-8 juice agar with and without $0.1 \%$ creosote was poured over the water agar plates. The creosote was used to selectively isolate creosote tolerant fungi (cf. Section II). 
In order to obtain data on the seasonal variation of $C$. resinae and other creosote tolerant fungi, the atmosphere around the storage tanks was continuously monitored by a seven day Kramer-Collins spore sampler (Kramer et a1., 1976) over a period of 24 months. The spore sampler was situated on the top of the cliff tanks, near the air inlet vent of the tank. In the laboratory the strips of double-sided cellotape coated with sterile vaseline were cut from the cassette and placed in sterile Petri-dishes. V-8 juice agar containing $0.1 \%$ creosote was poured over them and plates were then incubated at $25^{\circ} \mathrm{C}$ for 5 days before observation. Because the amount of air entering the trap and the rate of movement of the tape past the orifice is known, the concentration of the fungal spores in the air together with the time at which they landed can be calculated.

The soils were examined for the presence of $C$. resinae by the modified creosote matchstick method (Sheridan, Steel and Knox, 1971). The soils were collected from around the tanks into clean plastic bags using a sterile spoon. These soils were compared with a standard Brooklyn soil which had previously given positive results for the presence of $C$. resinae. All tests were set up in duplicate.

In November 1983, soil samples from the vicinity of the cliff tanks were collected (as above) and tested by the dilution plate method described below to determine the species of fungi present for comparison with those found in the fuel.

\subsubsection{Dilution plating}

5 grams from each of 22 soil samples were weighed accurately into $50 \mathrm{~cm}^{3}$ of sterile water and shaken on a mechanical shaker for 1 hour (100 rotation per minute). Nine dilutions from $10^{1}$ to $10^{9}$ were made in standard test-tubes. $1 \mathrm{~cm}^{3}$ aliquots from the undiluted suspension, the $\times 10, \times 10^{2}, \times 10^{5}$ and $\times 10^{9}$ dilution were separately added to $15 \mathrm{~cm}^{3}$ of cooled $\left(45^{\circ} \mathrm{C}\right)$ malt extract agar, contained in sterile $90 \mathrm{~mm}$ diameter plastic Petri-dishes. The dishes were rotated gently to disperse the spores in the agar. The colonies were observed after 5 or 6 days incubation at $25^{\circ} \mathrm{C}$. The Penicillium spp. isolated from the plates were re-inoculated into malt extract and Czapek-Dox agar media to identify the species. 


\section{$2.4 \quad$ Identification of micro-organisms}

Fungi were examined microscopically and identified using available keys. The Commonwealth Mycological Institute, Kew, England identified or confirmed identification of a number of species.

\subsection{Laboratory studies using fungi isolated from diesel fuel}

\subsubsection{Growth studies}

Growth studies of fungi isolated from fuel, air and soil in this study and of a range of fungi isolated from other sources were carried out using conidial suspensions as inoculum into aqueous/diesel phases. Growth and interactions were studied by observing the growth of mixed inocula and also by inoculating conidial suspensions into aqueous/diesel fuel phases recovered after six weeks growth of the same or different fungi. The selectivity of fungi was studied further by comparing growth obtained from diesel and aviation fuel as carbon source. In addition to growth measurements (dry weight) the survival of colony forming units (spores) or other fragments was also determined after the growth period.

(i) Single species culture (Table 2.1)

Growth was initiated by inoculating $100 \mathrm{~cm}^{3}$ of the culture media contained in a $200 \mathrm{~cm}^{3}$ medical flat bottle, with a spore suspension. The culture media varied between experiments, an initial set comparing diesel fuel alone with a $1: 1$ mixture of seawater/fuel, distilled water/fuel, tapwater/fuel and Bushnell-Haas mineral salt medium (BH)/fuel. Seawater was collected from the Zoology Marine laboratory, Wellington, left in the dark for several weeks before use in order to kill off any photosynthetic organisms and subsequent1y sterilized by filtration (using $0.22 \mu \mathrm{m}$ filters) in order to preserve the nutrient constitutents. Diesel fuel was also filter-sterilized. All the other aqueous media was autoclaved at $103 \mathrm{kPa}\left(121^{\circ} \mathrm{C}\right)$ for 15 minutes. 
Table 2.1 Growth Studies of single species in various media

\begin{tabular}{llll}
\hline Fungal & Diesel Seawater/ $/$ Distilled Tapwater/ BH/ BH/
\end{tabular}

Fuel only Diesel Water/Diesel Diesel Diesel Jet Fuel

1. N.Z. Isolates

C. resinae*

ex diesel

X $\quad X \quad X \quad X \quad X$

C. resinae*

ex soil (S82)

X X X X X

C. resinae* ex

air (A82)

$\mathrm{X}$

C. resinae

ex jet fuel (K10f)

X

Paecilomyces ${ }^{*}$

variotii ex diesel

(P82)

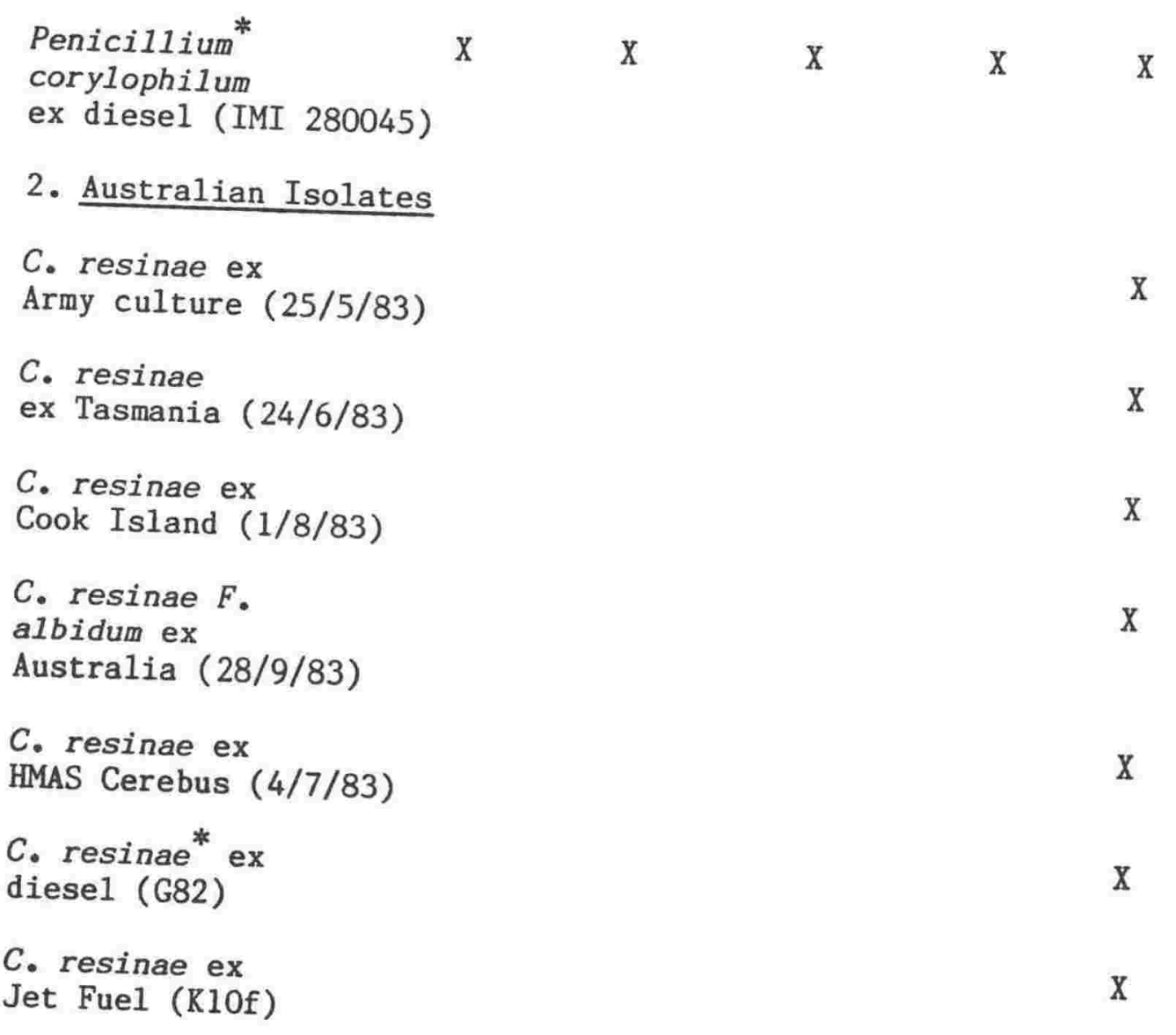

3. Penicillium spp. from diesel

P. chrysogenum *

(IMI277572)

X $\quad \mathrm{X}$

P. corylophilum *

P. echinulatum*

(IMI277570)

$x-x$

X $\quad$ X


Table 2.1(cont.) Growth Studies of single species in various media Fungal Species Growth Media Diesel Seawater/ Distilled Tapwater/ BH/ BH/ Fuel only Diesel Water/Diesel Diesel Diesel Jet Fuel

P. expansum *

(IMI282480)

P. digitatum * (IMI277577)

P. frequentans * (IMI277573)

P. spinulosum *

(IMI277571)

$\mathrm{X}$

$\mathrm{X}$

C. resinae*

ex diesel

C. resinae ex jet fuel (K10f)

Paecilomyces * variotii

4. Soil Isolates

Penicillium * X

(IMI282471)

P. brevicompactum ${ }^{*} \mathrm{X}$

P. funiculosum * X

P. 1oliense*

$\mathrm{X}$

X

(IMI282469)

$\mathrm{X}$

$\mathrm{X}$

(IMI282460)

$\mathrm{X}$

P. verrucosum ${ }^{*}$

$\mathrm{X}$

(IMI282470)

$\mathrm{X}$

$\mathrm{X}$

Trichoderma harzianum*

(IMI282478)

T. hamatum *

(IMI282475)

$\mathrm{X}$

$X$

* These species were isolated from this study 
The conidial suspensions were prepared from two week-old cultures grown on $\mathrm{V}-8$ juice agar at $25^{\circ} \mathrm{C} .20 \mathrm{~cm}^{3}$ of $\mathrm{BH}$ medium was shaken over the $\mathrm{V}-8$ juice agar culture and the resulting suspension centrifuged in a $15 \mathrm{~cm}^{3}$ sterile glass centrifuge tube at $1520 \mathrm{x} g$ for 20 minutes. The conidia were resuspended into $20 \mathrm{~cm}^{3} \mathrm{BH}$ medium and $0.1 \mathrm{~cm}^{3}$ containing approximately $5 \mathrm{x}$ $10^{6}$ spores $/ \mathrm{cm}^{3}$ was inoculated into the $\mathrm{BH} / \mathrm{fuel}$. The tests were set up in $200 \mathrm{~cm}^{3}$ medical flat bottles (previously sterilized in an oven at $200^{\circ} \mathrm{C}$ for 3 hours). The aluminium caps with the rubber liner removed, were screwed on loosely and the bottles incubated at $25^{\circ} \mathrm{C}$ for 6 weeks. At the end of this period, the growth was assessed visually. Regardless of growth or no growth, the contents of the bottles were filtered through $0.45 \mu \mathrm{m}$ membrane filters, partially wet in sterile water and diesel fuel. The filter membranes were dried to constant weight at $80^{\circ} \mathrm{C}$ in a hot-air oven. Constant weight was achieved in two days in the case of growths from diesel fuel. The dry weights of the membranes were recorded in mg. The $\mathrm{pH}$ of the aqueous phase was determined initially and at weekly intervals throughout the 12 weeks. (For each determination $5 \mathrm{~cm}^{3}$ of the aqueous phase was removed with a sterile pasteur pipette and returned to the remaining growth medium after $\mathrm{pH}$ determination).

\section{(ii) Growth in mixed cultures}

The same method used in (i) was used in the following growth experiments.

Two series of experiments were carried out to determine the effects of fungal growth upon the ability of media to support and maintain viability of subsequent spore inoculum.

(a) P. corylophilum ex diesel and $C$. resinae ex diesel were grown as mixed inoculum on seawater/Diesel(D. fuel), distilled water/D. fuel, tapwater/D. fuel, BushnellHaas/D. fuel and D. fuel only. After 6 weeks, growth was measured. Samples of $5 \mathrm{~cm}^{3}$ were removed from the interface and used for pour plate on V-8 juice medium and observed for growth. 
The aqueous phase was also examined in the light microscope to determine the extent of germination.

(b) Growth of $P$. corylophilum and Paecilomyces variotii was determined after 6 weeks growth in media which had previously been used for growth of C. resinae for 6 weeks. $C$. resinae was grown on seawater/D. fuel, distilled water/D. fuel, Bushne11-Haas/D. fuel at 1:1 ratio at room temperature, and harvested after 6 weeks and dry weights being recorded. The filtered media were then inoculated

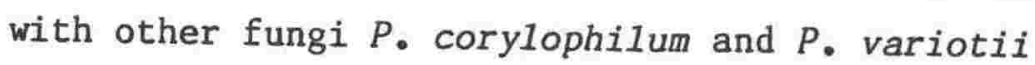
separately. The $\mathrm{pH}$ of the aqueous phase was determined initially and at weekly intervals throughout the 12 weeks. The initial $\mathrm{pH}$ of seawater was 8.3; of $\mathrm{BH}-7$, tapwater -7 and distilled water -7 . In order to establish whether germination had occurred, in doubtful cases, small amounts of water at the fue1-water interface were removed for microscopic examination. The microbial growth was observed visually and a rating system employed as follows:

0 - no germination nor growth

$+\quad$ - thin spore mat, very little growth

+ - very thin colourless mycelial mat

++ - thick dark mycelial mat.

(c) A second more extensive set of mixed spore cultures including more isolates were established as follows: Candida albicans (404) was grown. Alone and with each of the following fungi separately: C. resinae, Penicillium

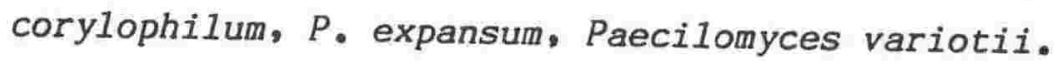

C. resinae was grown alone and with $P_{\bullet}$ corylophilum, $P$. expansum, and $P$. variotii.

P. corylophilum was grown alone and with $P$. expansum, and $P$. variotii.

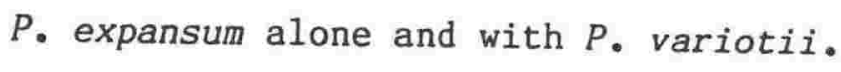




\section{P. variotii grown alone.}

The above fungi were grown for 6 weeks in seawater, tapwater, BH, seawater/Diesel, tapwater/Diese1, BH/Diesel. After 6 weeks, where there was no visual growth, the bottles were shaken thoroughly before removing $5 \mathrm{~cm}^{3}$ of the suspension for pour plate, to check the viability of the conidia. In the case of growth, the mycelial mat was harvested as mentioned previously and dry weights recorded and $\mathrm{pH}$ was monitored at weekly intervals.

(d) In a separate experiment medium in which $P$. corylophilum had grown for 6 weeks was inoculated with $C$. resinae, medium in which $C$. resinae had grown was inoculated with

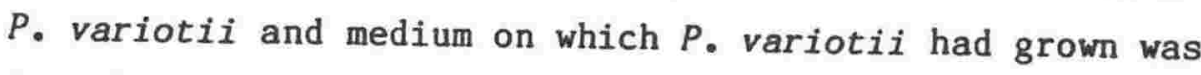
inoculated with $P$. corylophilum. Growth was measured after the first 6 weeks and the second 6 weeks of culture. The growth media were filtered before the second batch of inoculation. Growth media used were seawater/diesel fuel, seawater, tapwater/fuel, tapwater, $\mathrm{BH} / \mathrm{fuel}$ and $\mathrm{BH}$ only. $\mathrm{pH}$ was monitored weekly throughout the experiment.

\subsubsection{Effect of creosote on fungal growth}

Isolates from soil around the cliff tanks, diesel fuel in the tanks, Australian diesel, jet fuel and Cladosporium spp. from other sources were compared to determine their ability to grow in the presence of a range of creosote concentrations (table 2.2).

The concentrations of creosote used on V-8 juice agar were $0.05 \%$, $0.1 \%, 0.3 \%, 0.5 \%, 0.7 \%, 0.9 \%, 1 \%$. Agar plugs of $0.5 \mathrm{~mm}$ were taken from 7 day old V-8 juice agar cultures of the above fungi and inoculated centrally on the duplicate creosote plates. Plates were incubated at $25^{\circ} \mathrm{C}$ for 14 days. At two days interval, the growth diameter was measured. 


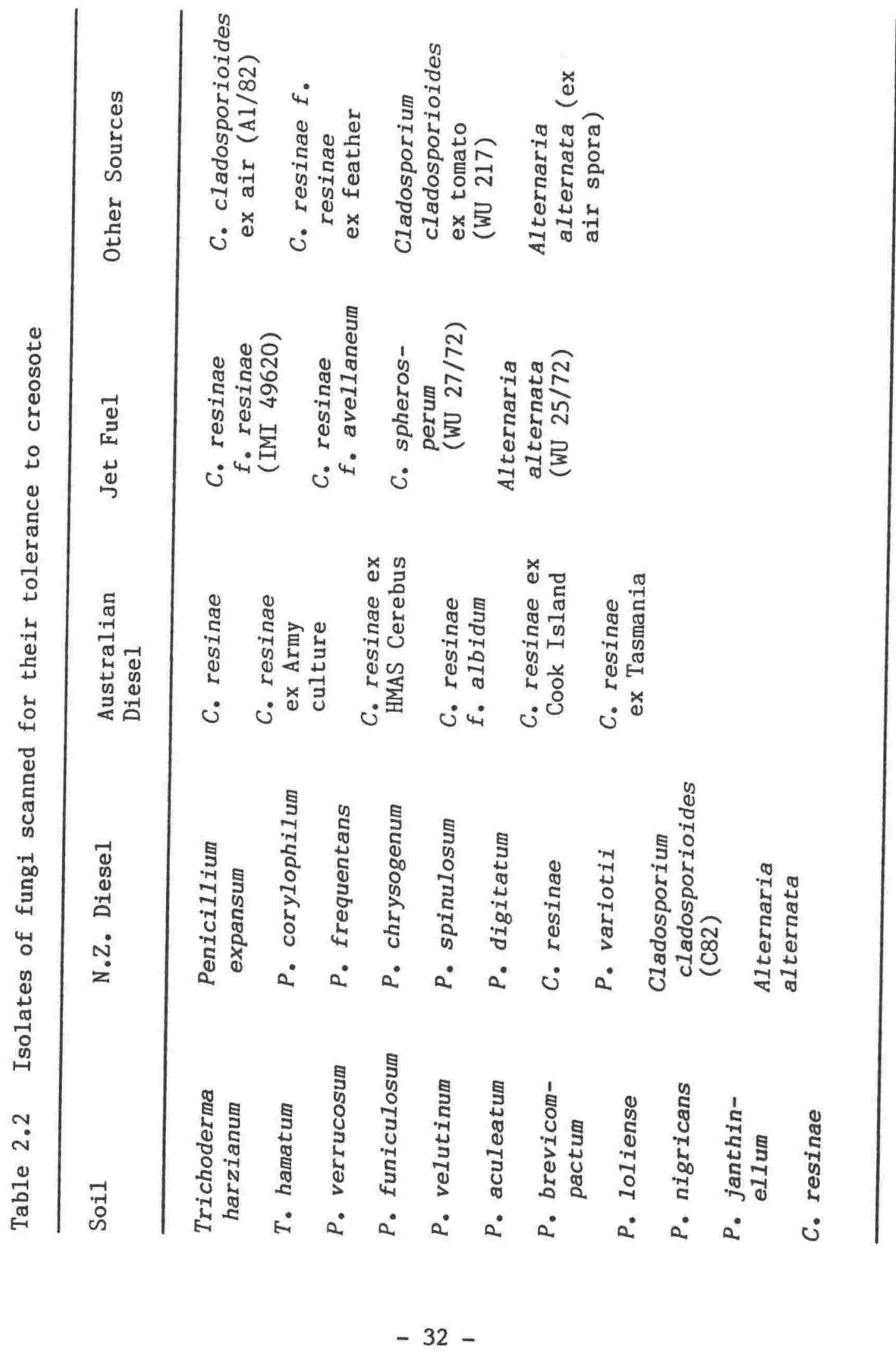




\subsubsection{Shaking and transport effects on spore viability in fuel}

The possibility that transporting fuel samples from Auckland to Wellington caused changes in the recovery of microbial contaminants, was examined in a series of simple tests.

(i) Filter-sterilized diesel fuel, $150 \mathrm{~cm}^{3}$ in sterile $200 \mathrm{~cm}^{3}$ medical flats was inoculated with spore suspensions in $\mathrm{BH}$ medium. $0.1 \mathrm{~cm}^{3}$ containing different concentrations $\left(5 \times 10^{2}\right.$ and $5 \times 10^{5}$ spore $/ \mathrm{cm}^{3}$ ) of $C$. resinae, $P$. corylophilum and $P$. variotii (all diesel isolates) singly and as a mixture of all three were added to a duplicate set of bottles. Each bottle was thoroughly hand shaken one hundred times and shaken by mechanical agitation for up to 8 hours, before reisolating the spores by filtration through a millipore filter $(0.45 \mu \mathrm{m}$ pore size). The filters were transferred aseptically to sterile Petri-dishes, covered with $\mathrm{V}-8$ juice agar and incubated at $25^{\circ} \mathrm{C}$ for 5 days.

(ii) Further samples of $C$. resinae were inoculated into filter sterilized fuels $\left(500 \mathrm{~cm}^{3}\right)$ in one litre tin cans and treated as indicated in table 2.3 in order to determine the effects of road transportation on viability.

Viable spores remaining were determined by membrane filtration and growth on V-8 juice agar as described above.

\subsection{Fine structure studies on hydrocarbon-utilizing fungi}

C. resinae $F$. avellaneum (ex jet fuel), C. resinae $F$. avellaneum, Paecilomyces variotii, Penicillium corylophilum (ex diesel fuel) and Cladosporium cladosporioides ex soil (V28) were used throughout this study. They were maintained on V-8 juice agar prior to examination. 5 day old cultures were used after growth at $25^{\circ} \mathrm{C}$.

\subsubsection{Light microscopy study}

Portions of a 5 day culture from the actively growing edge were mounted in lactophenol and photomicrographs were obtained using a Carl Zeiss plano-apo $x 40$ oil immersion objective on a photomicroscope. 
Table 2.3 The effects of road transportation on spore viability

Spore source and

concentration in inoculum

$\underline{\text { Treatment }}$

Replication

$\begin{array}{llll}\text { C. resinae ex diesel } & 37 \times 10^{6} / \mathrm{cm}^{3} & \text { Diesel fuel A } & \text { X5 } \\ \text { C. resinae ex diesel } & 37 \times 10^{6} / \mathrm{cm}^{3} & \text { Diesel fuel B } & \text { X5 } \\ \text { C. resinae ex diese1 } & 40 \times 10^{6} / \mathrm{cm}^{3} & \text { Diesel fuel C } & \text { X2 } \\ \text { C. resinae ex jet fuel } & 40 \times 10^{6} / \mathrm{cm}^{3} & \text { Diesel fuel C } & \text { X2 } \\ \text { C. resinae ex diesel } & 40 \times 10^{6} / \mathrm{cm}^{3} & \text { Kerosene C } & \text { X2 } \\ \text { C. resinae ex jet fuel } & 40 \times 10^{6} / \mathrm{cm}^{3} & \text { Kerosene C } & \text { X2 } \\ \text { C. resinae ex jet fuel } & 40 \times 10^{6} / \mathrm{cm}^{3} & \text { Diesel fuel D } & \text { X2 } \\ \text { C. resinae ex diesel } & 40 \times 10^{6} / \mathrm{cm}^{3} & \text { Kerosene D } & \text { X2 }\end{array}$

A - Tins were kept stationary in the laboratory over 3 days

B - Tins transported intermittently over $300 \mathrm{~km}$ during 3 days

C - Tins transported intermittently over $500 \mathrm{~km}$ during 2 days

D - replicates inoculated and filtered/analysed without shaking or transport. 


\subsubsection{Scanning electron microscopy (SEM)}

Because of the delicate nature of the above mentioned fungal material, it was necessary to use a preparatory procedure which preserved the original shape of the cells as well as the structural relationship between conidial chain and conidiogenous cells. The critical point drying method has demonstrated very good preservation of conidia and conidiogenous cells of deuteromycetous fungi (Cole, 1973, 1974, 1975, 1976) and has been used exclusively in this study.

A sporulating culture was first flooded with a mixture of equal volumes of $6 \%$ glutaraldehyde and $1 \%$ osmium tetroxide $\left(\mathrm{OsO}_{4}\right)$, each prepared at $4^{\circ} \mathrm{C}$ in $0.1 \mathrm{~m}$ sodium cacodylate buffer ( $\left.\mathrm{pH} 7.1-7.4\right)$. Small blocks (approximately $50 \mathrm{~mm}$ square) of agar and mycelium were then excised from the flooded culture and placed in a vial containing fresh, cold fixative solution and left in the dark for 2 hours. The samples were then washed in buffer ( 10 times) and placed in $1 \% \mathrm{OsO}_{4}$ buffered as above for 2 hours. This is considered to be an important step in the preparation of soft biological material for the SEM (Cole \& Samson, 1979). Kelly et a1. (1973) have shown that osmium forms a thin coat (40-200 $\mathrm{A}^{\circ}$ in thickness) over the surface of cells which provides the specimen with some resistance to contamination from electron beam during long term observation in the SEM. The specimens were subsequent1y washed in buffer (10 times) and then dehydrated in a graded acetone series $(30,50,70,90,95,100 \%)$.

The blocks of mycelium were then placed in Beem capsules whose ends were cut and replaced with fine mesh screens permitting free flow of liquid through the capsules while retaining the fungal samples. The Beem capsules were loaded with agar blocks in a small Petri-dish filled with absolute acetone. Extreme care was taken to prevent the specimens from drying out. The loaded capsules were then quickly placed in the bomb of the Polaran critical point drying apparatus and the latter was filled immediately with liquid carbon dioxide $\left(\mathrm{CO}_{2}\right)$. The bomb was flushed with liquid $\mathrm{CO}_{2}$ for 2 minutes and then filled and sealed off for 1 hour. It was then partially emptied (pressure in bomb reduced to approximately 250 p.s.i.). Finally, the bomb was sealed and its pressure increased to $1200 \mathrm{p} . \mathrm{s} . i$. by heating with water at approximately $32^{\circ} \mathrm{C}$. 
The $\mathrm{CO}_{2}$ gas was subsequently released and the dried specimens were removed from the Beem capsules and mounted on aluminium stubs which were coated with conductive silver paint. The stubs were placed on an omnirotary table in a Dynavac Sputter Coater Model Sc 150 high vacuum evaporator and coated with about $100 \mathrm{~A}^{\circ}$ (Russ and Kabaya, 1970) of gold palladium. The material was examined in a Phillips SEM 505. The scanning electron micrographs were recorded with PAN-F medium speed film.

\subsubsection{Transmission electron microscopy (TEM)}

C. resinae (ex jet and diesel) and C. cladosporioides (ex soil) cultures were used for this study. Small blocks (approximately 5-10 $\mathrm{mm}$ square) of agar and mycelium were excised from culture. A number of fixation procedures were used in this study and these are detailed on table 2.4 .

After fixation, the material was dehydrated in an ethanol series $(30,40,50,70,90,95,100 \%)$. Routinely, specimens were post-stained in $2 \%$ uranyl acetate in the $70 \%$ ethanol stage during dehydration for 45 minutes. The material was then placed in $25 \%$ Spurr's low viscosity resin (1969) (obtained from Polyscience Inc.) in absolute ethanol for 1 hour, with continuous, gentle agitation on a rotating stage. The samples were then transferred to a $50 \%$ resin solution for 4 hours, to $80 \%$ resin for 4 hours and $100 \%$ resin overnight. The material was then removed from the vials, placed in fresh $100 \%$ resin in capsules and allowed to polymerise at $70^{\circ} \mathrm{C}$ overnight.

The specimens were sectioned using standard techniques on an LKB8800 ultratome III. Thin sections were picked up on copper grids (400 mesh). Before electron microscopy, grid mounted sections were further stained with $0.5 \%$ aqueous uranyl acetate (20 minutes) and Reynold's (1963) lead citrate 7 minutes. The material was examined in a Philips EM420 twin Transmission Electron Microscope (TEM). 


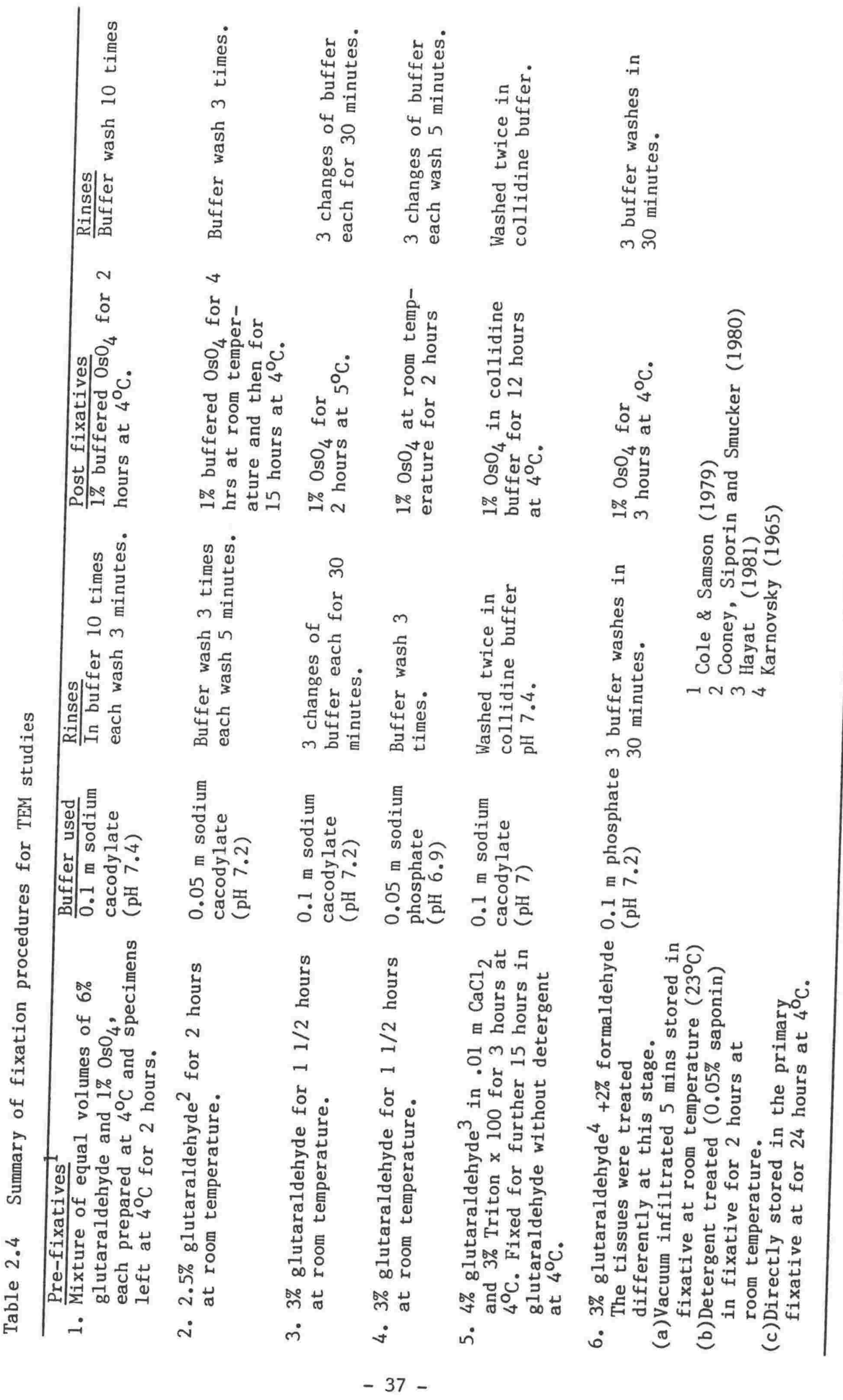




\subsection{Determination of water in diesel fuel samples}

The total water (free and dissolved water) in the fuel sample was determined at the Defence Scientific Establishment by the Karl Fisher method (ASTMD 1744-64).

\subsection{Meteorological records}

A Thermohygrograph was set up within the entrance corridor leading to Cliff tank 2 to provide a continuous record of temperature and humidity during these studies. Rainfall data for Auckland was obtained from the Metereological Office at Wellington. 


\section{EXPERIMENTAL RESULTS : SECTION I - FIELD STUDIES}

\subsection{Micro-organisms occurring in diesel fuel.}

Thirty-one species of viable fungi were isolated from 'dieso' fuel samples collected from fuel storage tanks at the naval base at Devonport, Auckland. Most belonged to the Hyphomycetes but Zygomycetes, Ascomycetes and Basidiomycetes were also represented. Only five bacterial isolates were obtained. These included a sulphurreducing bacterium. Fungi and bacteria isolated and identified over a 24 month period are listed in table 3.1.

Tables 3.2 and 3.3 present a summation of the quantitative data (refer appendices $3.1,3.2$ and 3.3), obtained from the assay of the monthly samples for each of the two storage tanks, the separating tank, the Shell tank and the wharf sampling point. Mean numbers of CFU were obtained for each fungus and for each separate position analysed. The tables also list the frequency of isolation of each fungus as a percentage of the total samples analysed.

The most frequently isolated contaminants were the fungi Cladosporium resinae, Penicillium corylophilum and Paecilomyces variotii (table 3.2). These were isolated from both storage tanks at each monthly sampling date over the 24 month period. Tank 3 was more heavily contaminated than tank 2. The mean number of CFU tended to be higher at the bottom or 'very bottom' of the tanks. The above three fungi were also the predominant contaminants found in the fuel samples from the separating tank, wharf sampling point, Shell tank and tank cleaning vessel (table 3.3). The highest mean number of CFU for C. resinae were found in the tank cleaning vessel (372 CFU/1itre) and Shell tank sample ( $320 \mathrm{CFU} / 1$ itre). 
Table $3.1 \quad \begin{aligned} & \text { Fungi isolated and identified from N.Z. stored diesel fuel } \\ & (1982-1984)\end{aligned}$

\section{ZYGOMYCETES}

Mucor sp.

Rhizopus sp.

ASCOMYCETES

Amorphotheca resinae Parbery

Chaetomium globosum Kunze

\section{BASIDIOMYCETES}

Unidentified isolates with clamp connexions.

HYPHOMYCETES

Alternaria alternata ( $\mathrm{Fr}$. ) Keissler

Aureobasidium pullulans (de Bary) Arnaud

Aspergillus fumigatus* Fres.

Aspergillus sejunctus* Bainier and Sartory

Aspergillus versicolor* (Vuill.) Tiraboschi

Aspergillus niger Van Tiegh

Botrytis sp.

Cladosporium resinae (Lindau) de Vries state of Amorphotheca resinae.

Cladosporium cladosporioides (Fresen.) de Vries

Epicoccum purpurascens Ehrenb. ex Schlecht

Fusarium sp.

Gliocladium roseum Corda

Paecilomyces variotii Bainier

Paecilomyces 1ilacinus* (Thom) Samson

Penicillium corylophilum* Dierckx

Penicillium digitatum* (Pers. ex Fr。) Sacc.

Penicillium echinulatum* Raper \& Thom ex Fassatiova

Penicillium chrysogenum* Thom

Penicillium frequentans* Westling

Penicillium spinulosum* Thom

Penicillium expansum* Link

Phomopsis sp.

Trichoderma viride Pers. ex S.F. Gray

Trichoderma hamatum* (Bonorden) Bainier

Trichoderma harzianum* Rifai

Ulocladium sp.

Yeasts

Bacteria

Bacillus sp.

Pseudomonas sp.

Micrococcus sp.

Desulfovibrio desulfuricans

* Identified or identification confirmed by the Commonwealth Mycological Institute, Kew, England. 


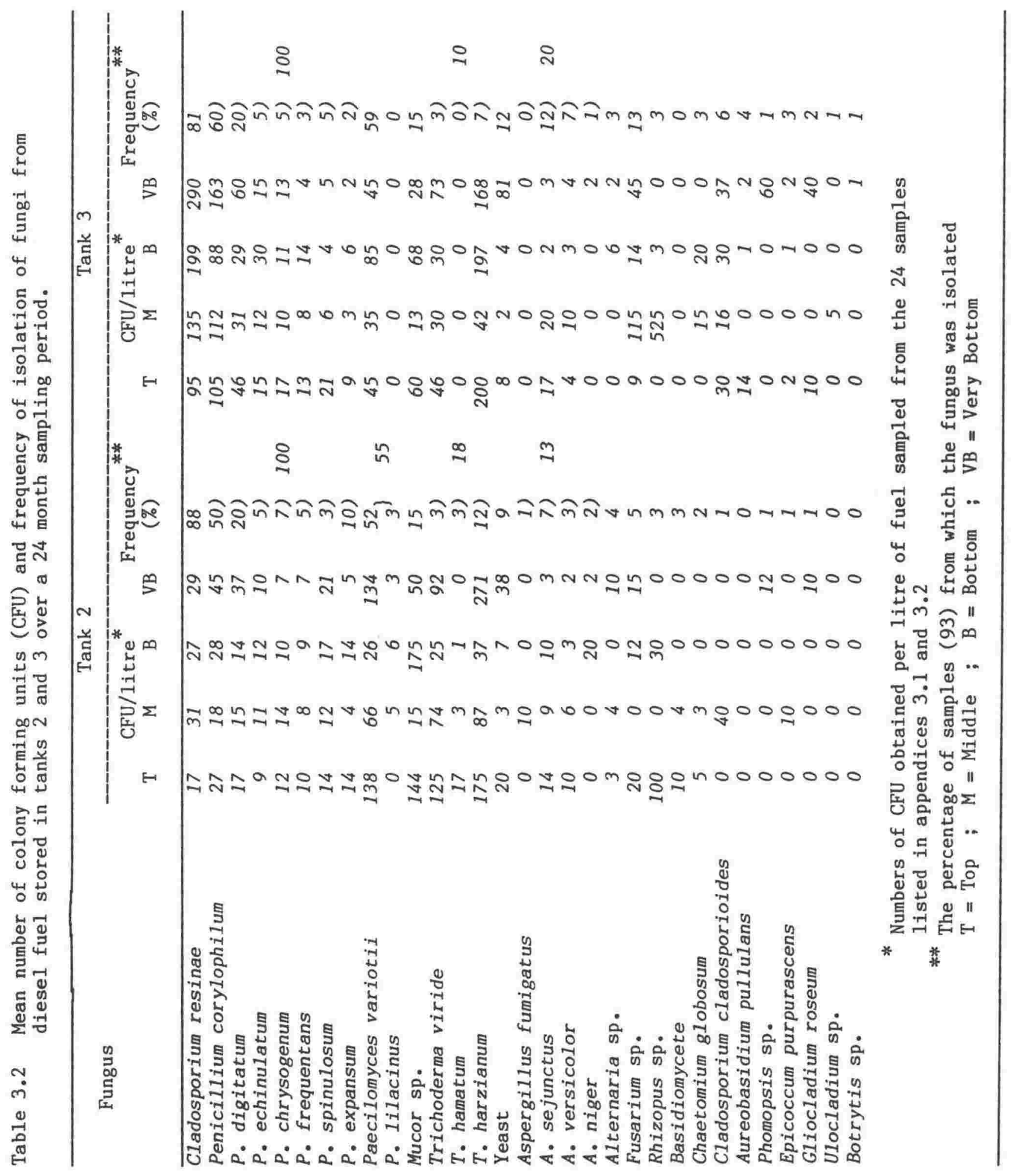


Table 3.3 Mean Number of Colony Forming Units (CFU) and frequency of isolation of fungi from the separating tank, Shell tank, tank cleaning vessel and wharf sampling point

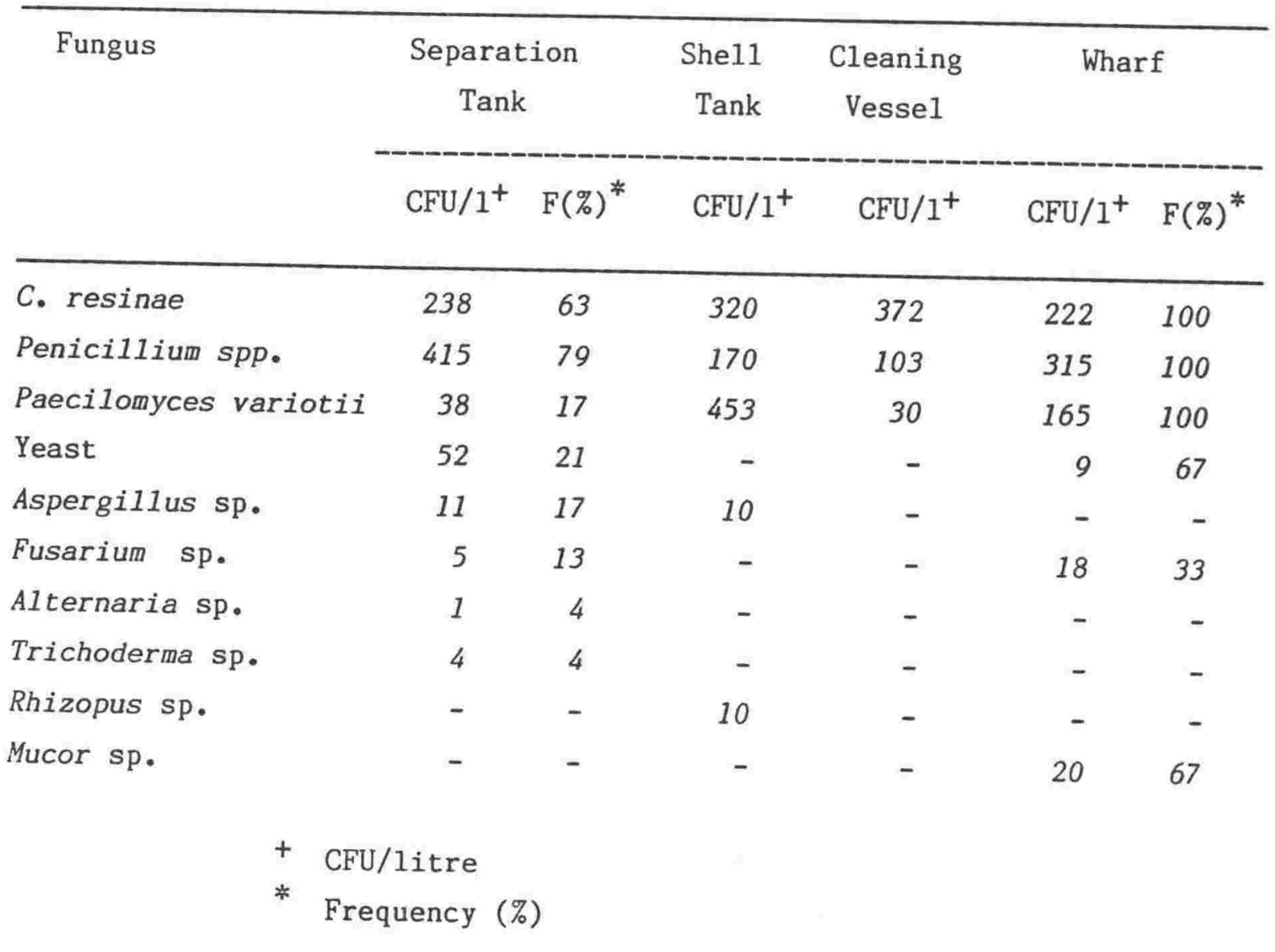

The total water (dissolved and free water) was usually more than $130 \mathrm{ppm}$ in tank 3 while tank 2 had less than $105 \mathrm{ppm}$. Sometimes three-quarters of the 'very bottom' of tank 3 sample and the separating tank sample contained water. With excessive amounts of water, the $\mathrm{pH}$ usually exceeded 8 and the conditions became more favourable for anaerobic bacteria. During the day the flow of fuel in the separating tank from all the cliff tanks was monitored and only fuel was pumped back into tanks. At night, however, both fuel and water were automatically pumped into tank 3 causing an increase in the water level and possibly contamination levels. 
Seven species of Penicillium were isolated from the storage

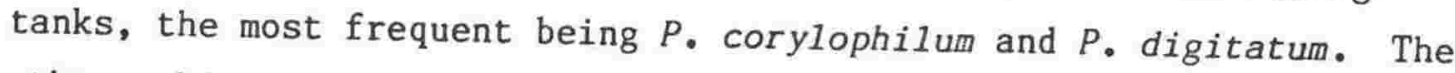
others which occurred less frequently and in low numbers were $P$. echinulatum, $P$. chrysogenum, $P$. frequentans, $P$. spinulosum and $P$. expansum. Paecilomyces variotii occurred more frequently than P. lilacinus. Trichoderma viride, $T$. hamatum and $T$. harzianum were found occasionally in the tanks when the predominant fungi were in low numbers. They did not grow in fuel but remained as viable spores (see section II).

The data in figures $3.1 \mathrm{~A}$ and $3.1 \mathrm{~B}$ show the fluctuation of temperature, humidity and the major fungi in the storage tanks over the twenty-four months. The air temperature $\left(15^{\circ} \mathrm{C}-20^{\circ} \mathrm{C}\right)$ and the humidity ( $70-95 \%)$ were ideal for the growth of most of the fuel fungi. The surface fuel temperature was the same as the surrounding air temperature and it was almost constant with depth in the tank apart from an occasional difference of $1^{\circ} \mathrm{C}$.

No regular seasonal pattern was observed for the predominant fungi. C. resinae, occurred in greater numbers between May '82 April ' 83 and December '83 - February '84 while $P$. variotii occurred frequently in low numbers compared to $C$. resinae and Penicillium spp. Penicillium spp. numbers were consistent throughout the two years of sampling and peaked during September '84.

Figures $3.2 \mathrm{~A}, \mathrm{~B}$ and $\mathrm{C}$ shows the variation in the $\mathrm{CFU}$ of the three major fungi in the separating tank, and tanks 2 and 3 over twelve months. Between July 1982 and September 1983, the separating tank was contaminated with high numbers of $C$. resinae which decreased during the subsequent months. During the same period, the CFU of C. resinae in tank 3 were low initially and then increased in number. In tank 2, C. resinae remained low compared to the other two tanks. Penicillium spp. were persistent in all the tanks.

Bacteria occurred in low numbers in the diesel fuel. Sulphur bacteria became a problem when the very bottom of tank 3 and the separating tank contained an excessive amount of water. 


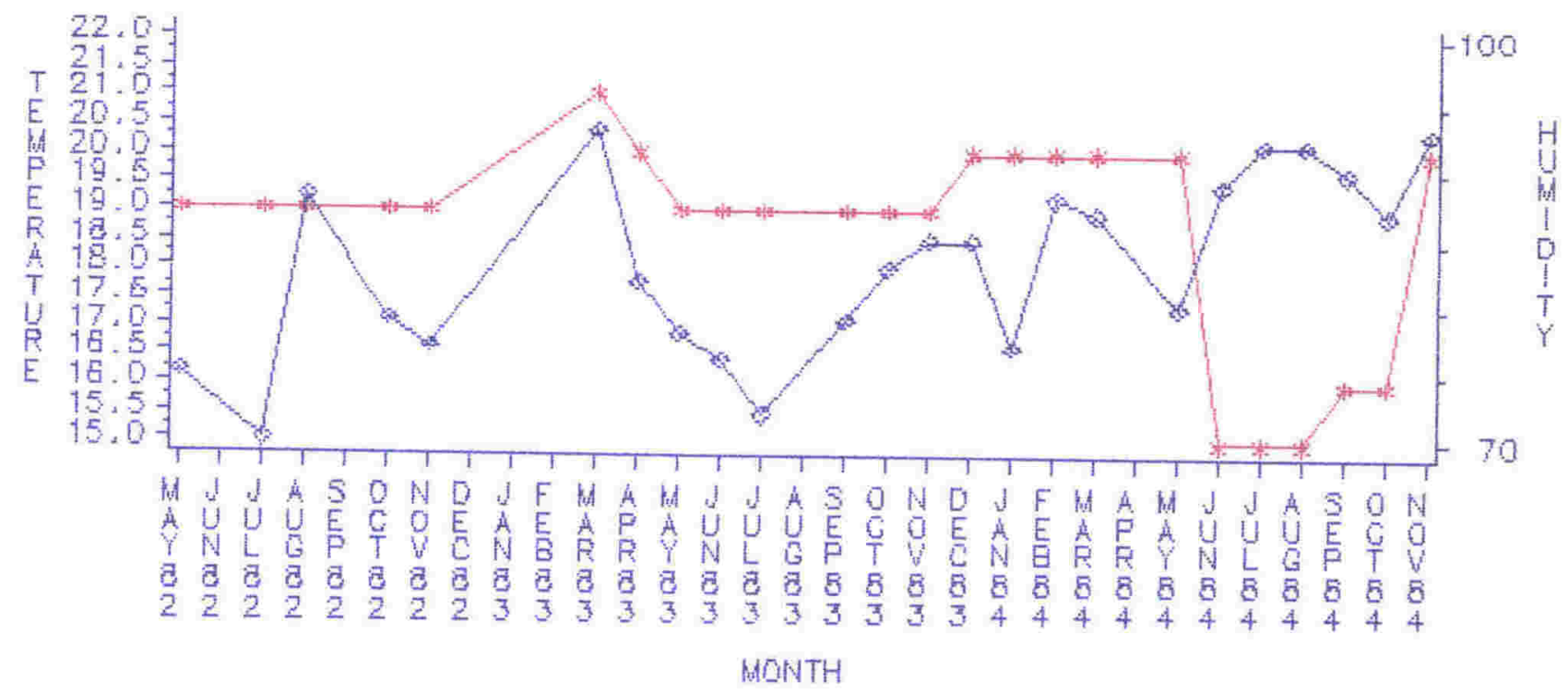

\section{FREQ}

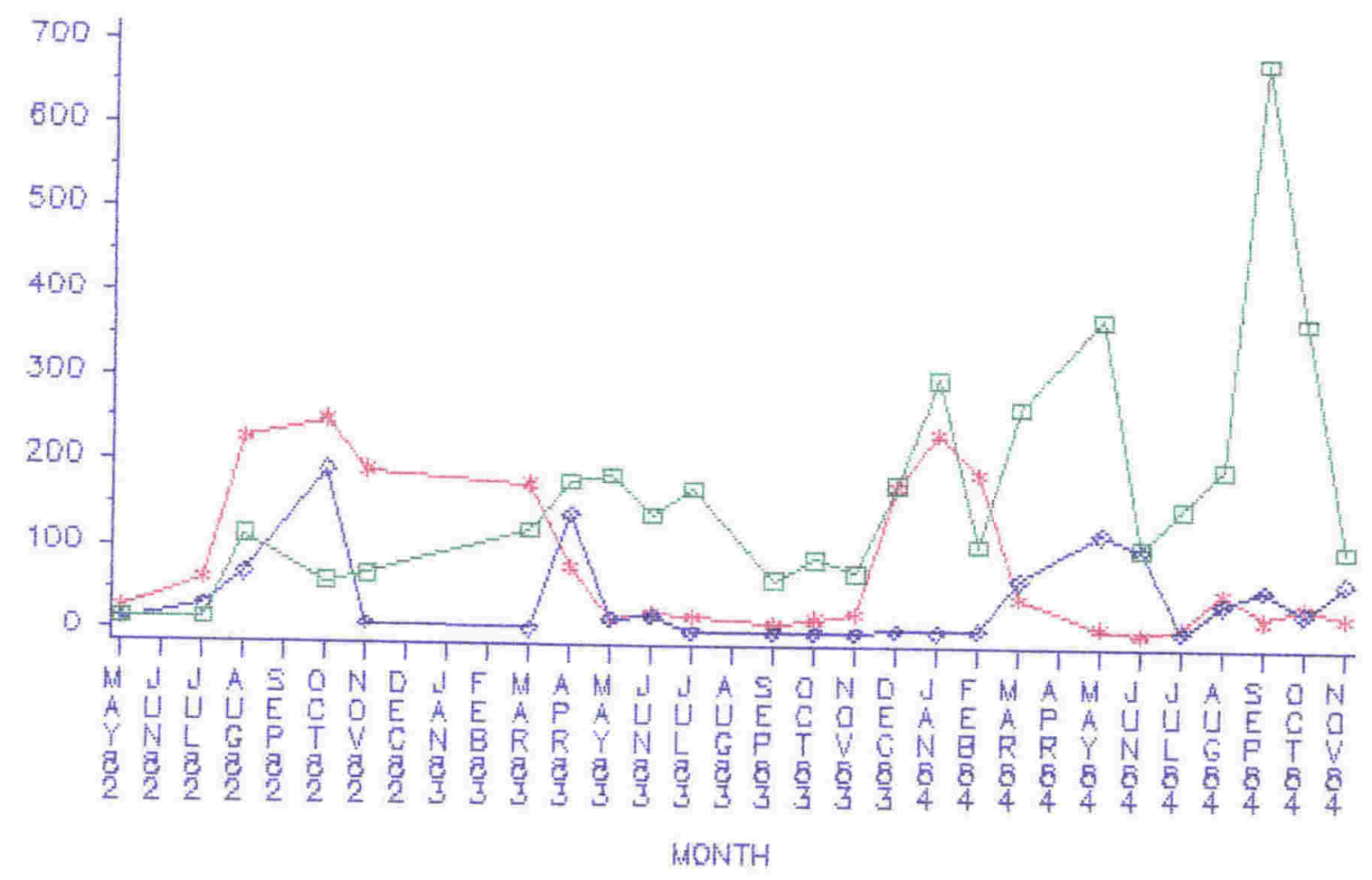

TYPE * C.RESINAE $\Xi \square \square$ PENTCILLTLA SPP 
Figure 3.2 Comparing the occurrence of the predominant fungi in the separating tank with that of tanks 2 and 3 (1982-1984)

A. Mean number of CFU/litre of fungi occurring in the separating tank

B. Mean number of CFU/1itre of fungi occurring in the storage tank 2 (page 46)

C. Mean number of CFU/litre of fungi occurring in the storage tank 3 (page 47) 


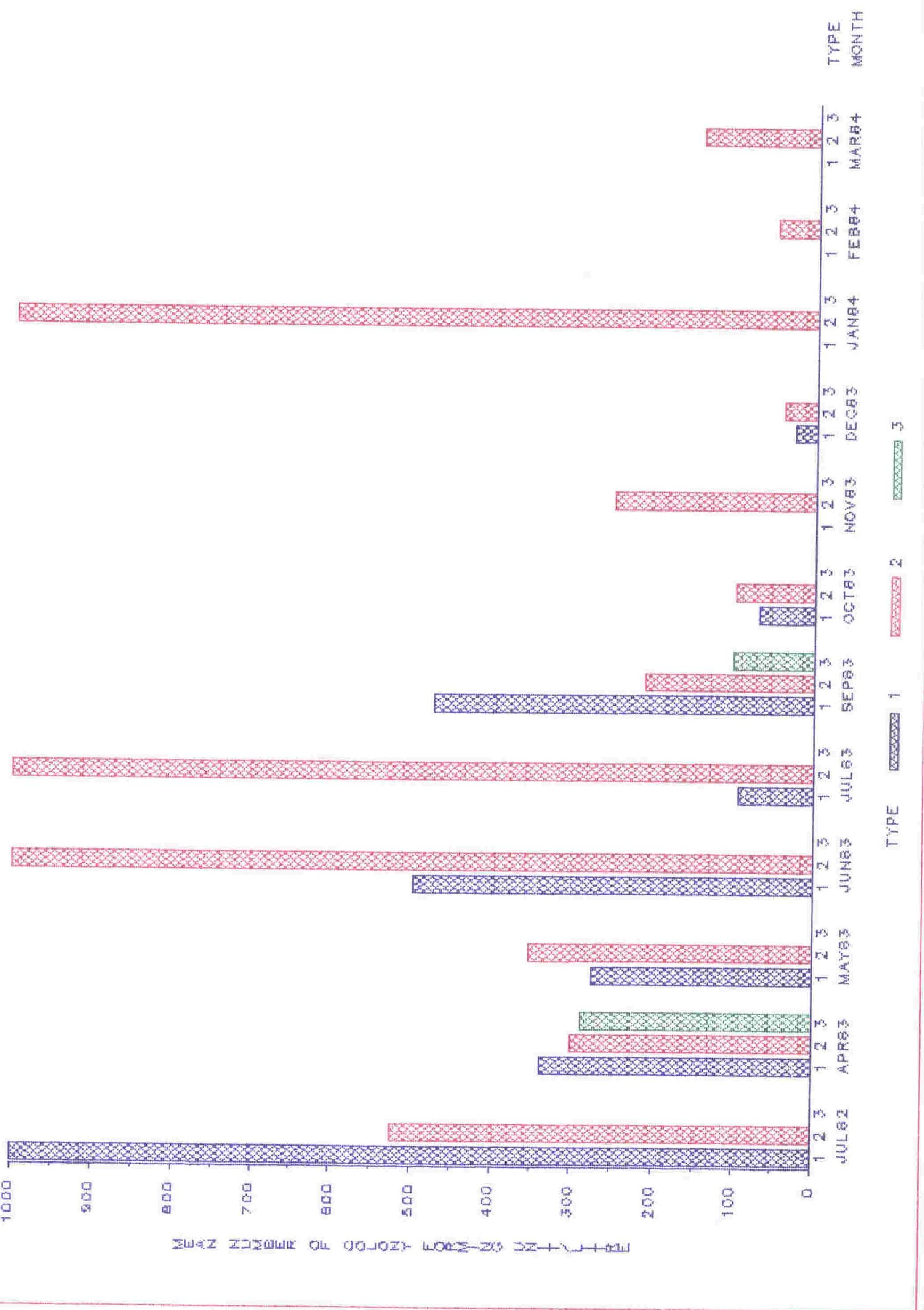


\#3\%
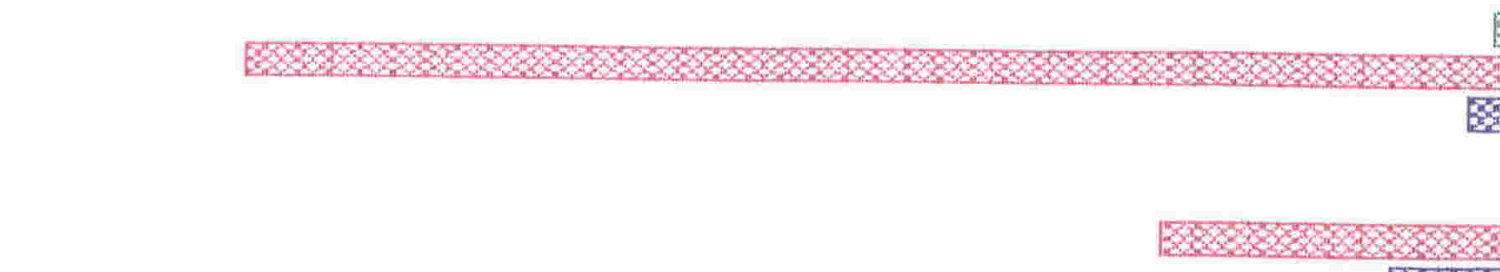

ris

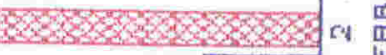

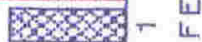

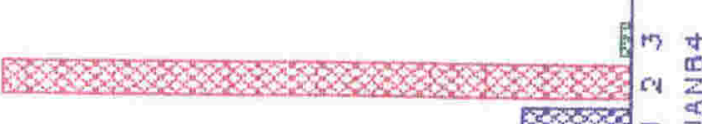

$3 \mathrm{~m}$

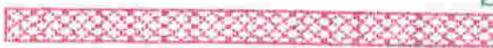

त 苍

Bस8\%8

w.

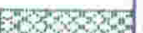

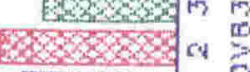

ए8F - -

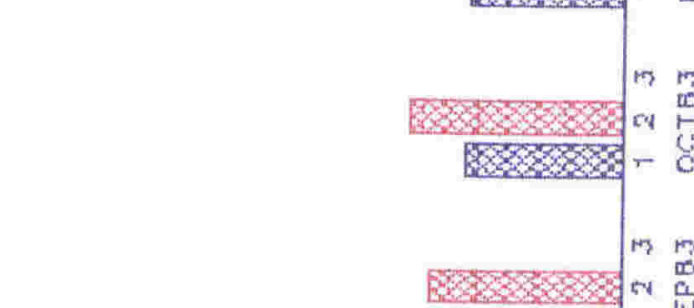

c大oxa- 4

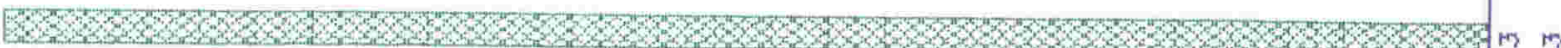

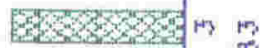

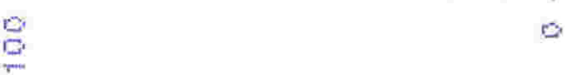

ZUA I 


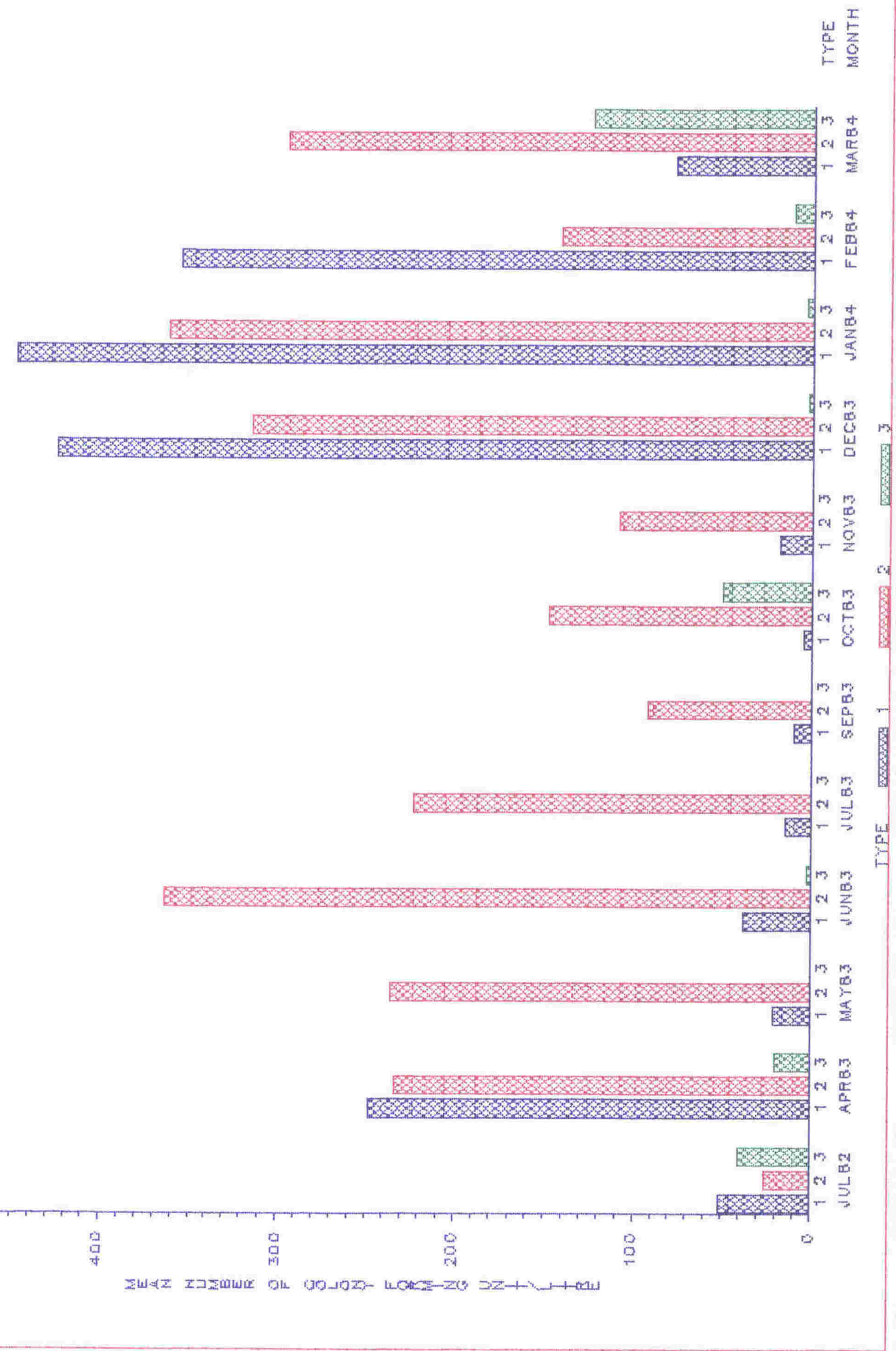


The commonly isolated bacteria were Pseudomonas spp., Micrococcus spp. and Bacillus spp. (table 3.4 and 3.5). The only sulphur-reducing bacterium isolated was Desulfovibrio desulfuricans from the water bottoms. Isolation of this organism coincided with an obvious smell of hydrogen sulfide emanating from the fuel. On Baar's medium and API broth and agar the presence of Desulfovibrio was indicated by the medium turning black. Attempts to isolate $E$. coli failed indicating that sewage contamination of the stored fuel had not occurred. Colonies on MacConkey medium were sticky and showed confluent growth. The organisms were Gram negative, rod-shaped, non-sporing and nonlactose fermenting and therefore not $E$. coli. The number of bacteria occurring in diesel fuel decreased with storage.

Table 3.4 Incidence and levels of bacteria found in diesel fuel at different testing dates.

\begin{tabular}{|c|c|c|c|c|c|c|}
\hline \multirow[b]{2}{*}{$\begin{array}{l}\text { Date and time } \\
\text { of sampling }\end{array}$} & \multicolumn{6}{|c|}{ Number of Bacterial Colonies per litre } \\
\hline & \multicolumn{2}{|c|}{ Micrococcus sp. } & Pseudomonas sp. & $\begin{array}{l}\text { nas sp. } \\
\text { Tank } 3\end{array}$ & \multicolumn{2}{|c|}{ Bacillus sp. } \\
\hline \multicolumn{7}{|l|}{ July 1982} \\
\hline Immediate & 0 & 0 & 0 & 0 & 1 & 22 \\
\hline 24 Hours later & 4 & 0 & 0 & 0 & 20 & 13 \\
\hline
\end{tabular}

August 1982

\begin{tabular}{lrrrrrr} 
Immediate & 142 & 60 & 0 & 0 & 0 & 52 \\
24 Hours later & 40 & 72 & 27 & 0 & 30 & 67 \\
48 Hours later & 107 & 93 & 7 & 0 & 67 & 0 \\
72 Hours later & 27 & 27 & 0 & 0 & 7 & 0 \\
96 Hours later & 53 & 0 & 0 & 0 & 7 & 0 \\
\hline
\end{tabular}




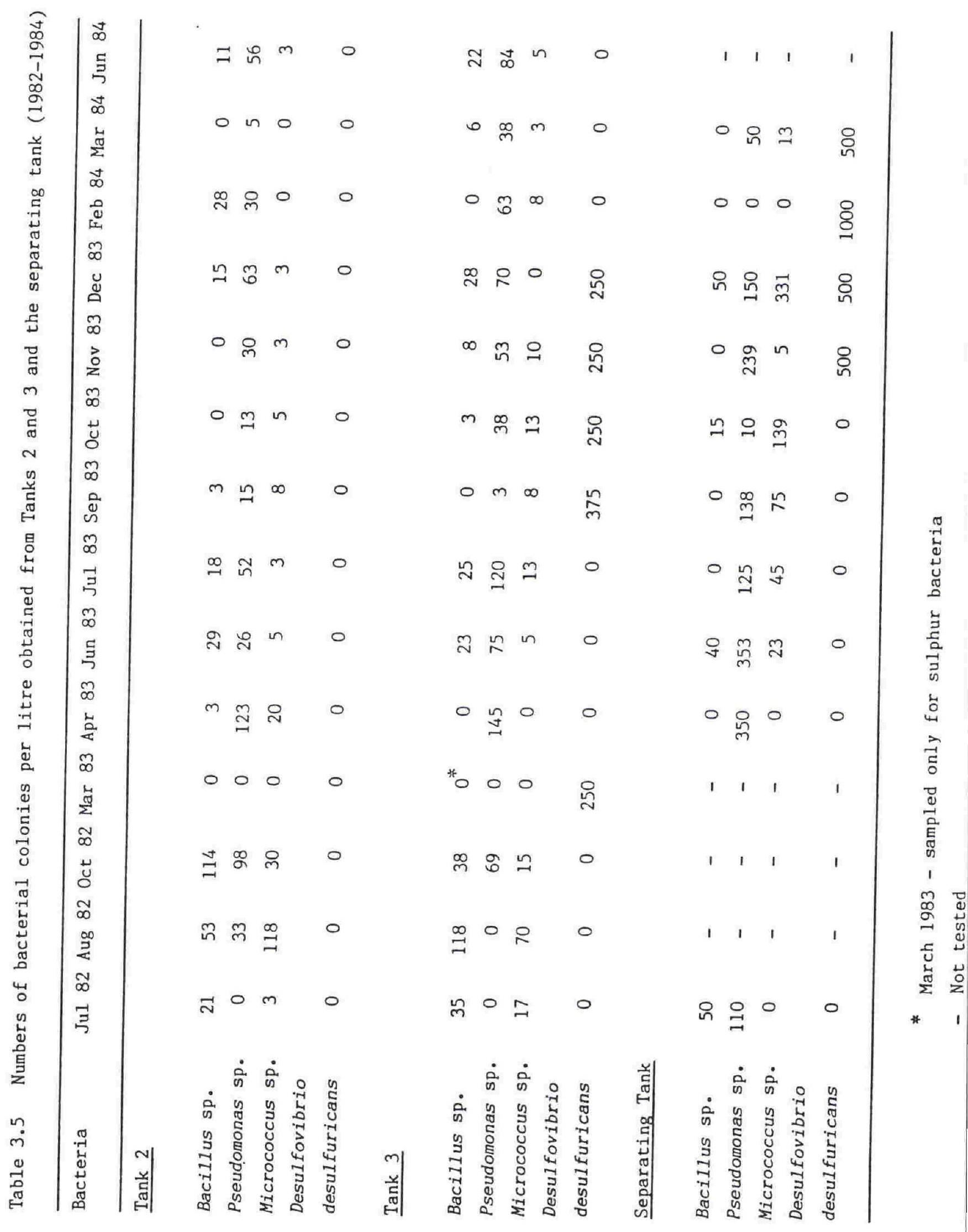




\subsection{Comparison of isolation methods}

Direct observation of filters showed a wide variety of fungi existing in fuel including Alternaria, Drechslera, Epicoccum, Trichoderma and Ulocladium and many unidentified spores. This method was tedious because of the large amounts of inert non-biological material present and the difficulties of distinguishing fungal spores. Furthermore the majority of these fungi failed to sporulate on culture plates.

Pour and spread plate methods were used when fuel samples were saturated with water or contained a distinct water layer. As a routine the membrane filtration technique was used because it allowed both qualitative and quantitative studies. A number of modifications were examined. The one giving consistently highest counts was adopted i.e. medium poured over the filter membrane (see table 3.6). V-8 juice agar medium gave reliable and consistent results and was used throughout.

Table 3.6 Comparison of two media and various modified membrane filter techniques for two separate fuel samples. (10 replicates)

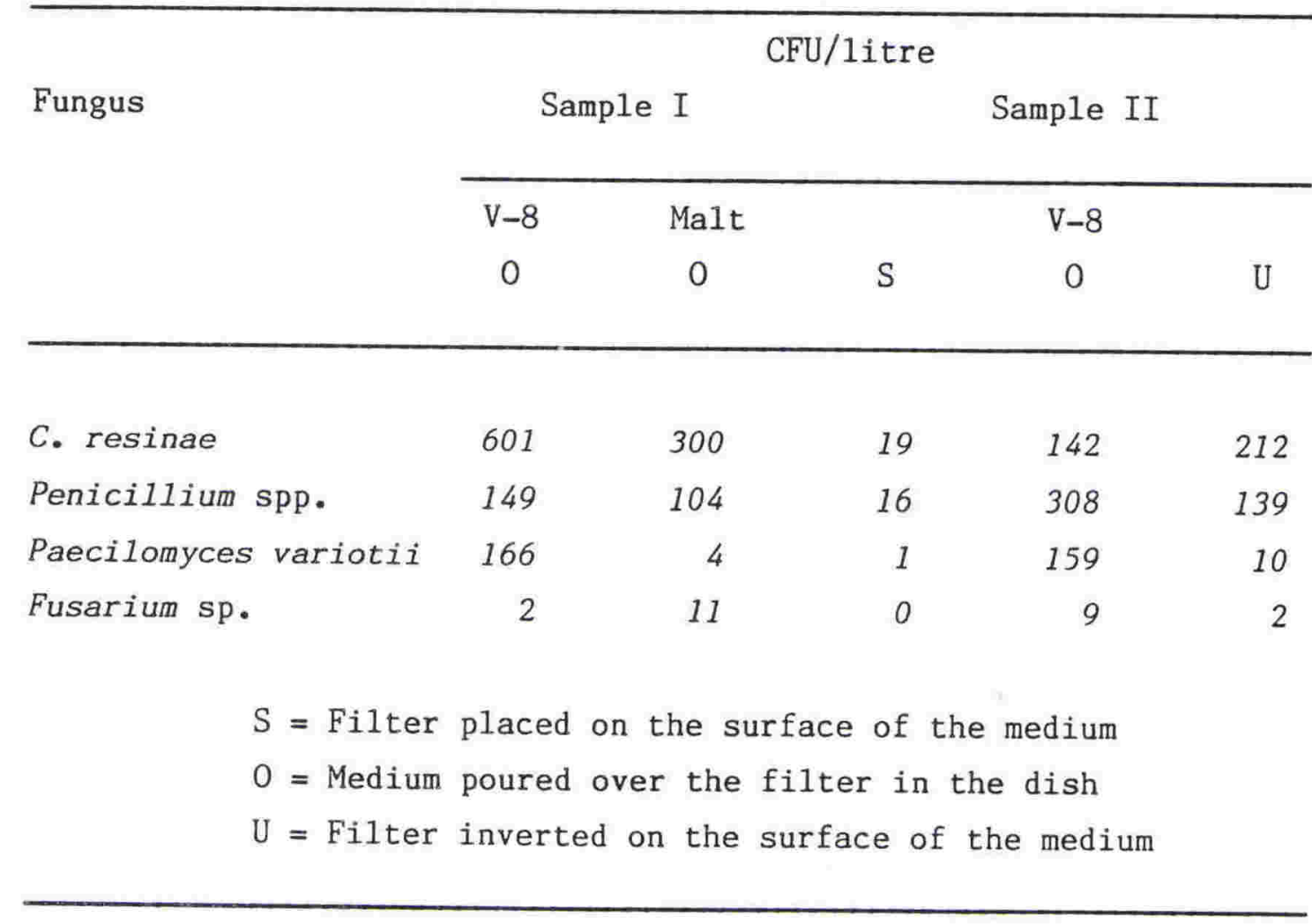


3.3 Comparison of container type used for collecting samples

(Table 3.7)

Generally the bottle counts of $C$. resinae were lower than the counts from tin stored samples. The CFU for both container types fluctuated during the different sampling period. However, the former were preferred because they do not corrode and are easier to sterilize.

\subsection{Effect of time of collection and testing of samples on the recovery of $C$. resinae (Table 3.7 )}

Initial tests were carried out with a minimum delay to avoid changes in the composition and relative abundance of the microflora. Subsequent tests were carried out at intervals up to five days after collection. In jet fuel, delayed testing resulted in failure to recover C. resinae (Sheridan pers. comm.). The July '82 samples were tested immediately and after 24 hours only. Mean counts of $C$. resinae in tank 2 decreased from $62 \mathrm{CFU} / 1$ itre to $28 \mathrm{CFU} / 1$ itre within 24 hours. The apparent drop in tank 3 was due to very low figure obtained for the bottle samples which were not tested immediately after collection. In the August sampling this drop did not occur. Further sampling in October and November showed less marked differences on the recovery of C. resinae within each tank, with the different time of testing.

\subsection{Sources of fuel contamination}

The occurrence of C. resinae, Penicillium spp. and Paecilomyces variotii in the surrounding atmosphere near the tanks can be seen in figures $3.3 \mathrm{~A}, \mathrm{~B}$ and $C_{\text {. }} P_{\text {. corylophilum, }} P_{\bullet}$. digitatum and $P_{\text {. expansum }}$ frequently occurred in the air spora and showed a corresponding high incidence in the fuel storage tanks. But no such observation was made for $C$. resinae and Paecilomyces variotii. Most of the $C$. resinae and $P$. variotii spores were trapped in summer and autumn and occurred in small numbers. In tank 2 among the three fungi Penicillium spp. and P. variotii showed high peaks in April 1983 and May 1984 after low numbers in the preceding months. 
Table 3.7 Effect of time of testing of samples and container types on recovery of $C$. resinae

$\mathrm{CFU} / 1$ itre

Date and time

of testing Tank $2^{+}$Tank $3^{+}$Wharf $\operatorname{Tin}^{*}$ Bottle

July 1982

Immediate

24 Hours later

$\begin{array}{rrrrc}62 & 1000 & 1000 & 598 & - \\ 28 & 533 & 536 & 451 & 183\end{array}$

August 1982

Immediate
24 Hours later
48 Hours later
72 Hours later

25

225

115

124

$25 \quad 307$

458

178

237

$173 \quad 351$

510

546

282

$70 \quad 321$

25

212

111

73

438

28

286

160

October 1982

24 Hours later

48 Hours later

13

288

285

231

162

72 Hours later

7

397

255

231

163

96 Hours later

10

403

215

227

165

120 Hours later

13

410

295

257

162

62

745

630

422

461

November 1982

24 Hours later

48 Hours later

72 Hours later

96 Hours later

120 Hours later

$\begin{array}{rl}23 & 372 \\ 15 & 246 \\ 7 & 295 \\ 11 & 513 \\ 7 & 111\end{array}$

$\begin{array}{lr}- & 209 \\ - & 158 \\ - & 183 \\ - & 347 \\ - & 99\end{array}$

246

136

163

253

$99 \quad 14$

- not tested

* mean for tanks 2 and 3

+ mean for tin and bottle samples 
Figure 3.3 The occurrence of the predominant fungi

in the atmosphere and the storage tanks 2 and 3 (1982-1984)

A. Mean number of colonies/m $/ \mathrm{m}^{3}$ of fungi occurring in the air spora

B. Mean number of CFU/1itre of fungi occurring in the storage tank 2

C. Mean number of CFU/litre of fungi occurring in the storage tank 3 
AIR SPOR

OOLDNY

130
100
50
0

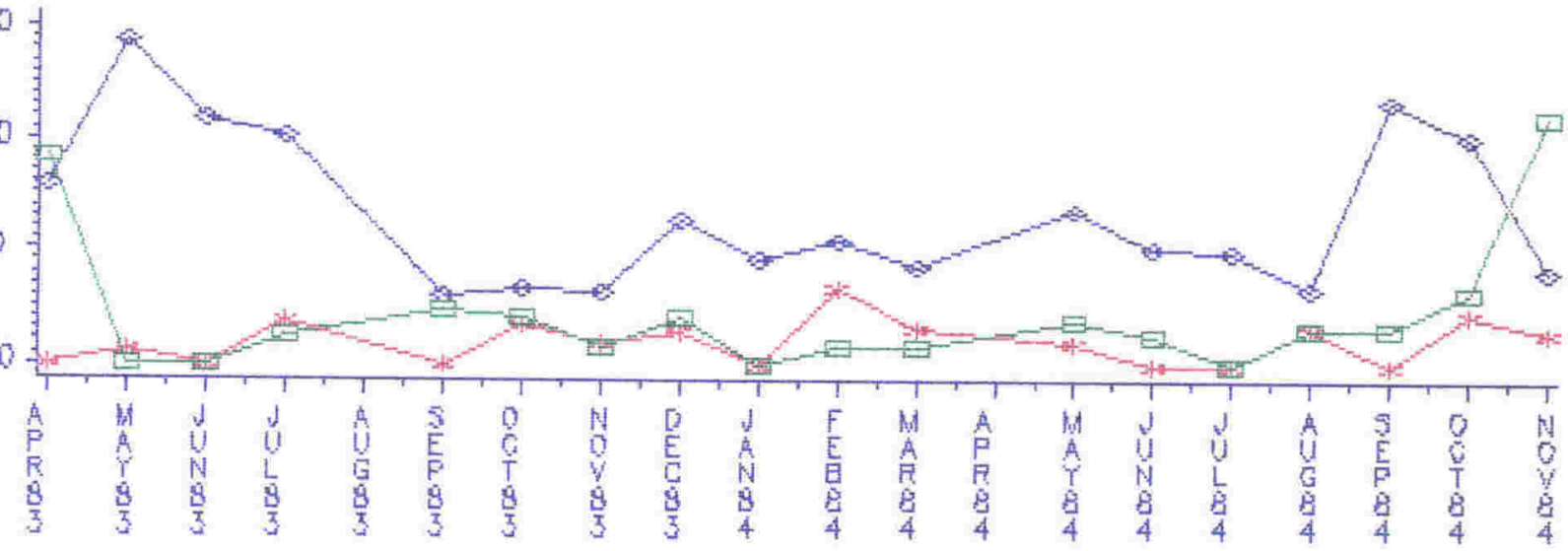
WONTH

TTPE HESINAE $\Leftrightarrow \Leftrightarrow P$. CORYLOPHILLM EEEP. VAFIOTII

TANK2

CoLONO

600
500
400
300
200
100

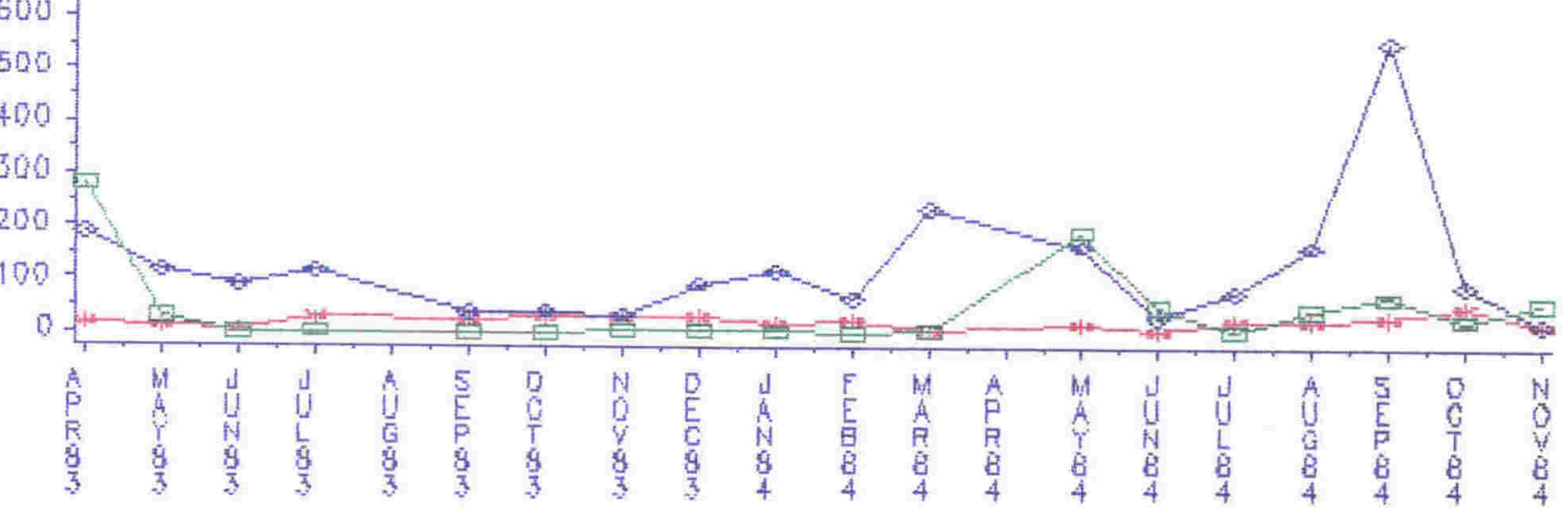

MONTH

TYPE the RESIMAE

TANGI

COLONY

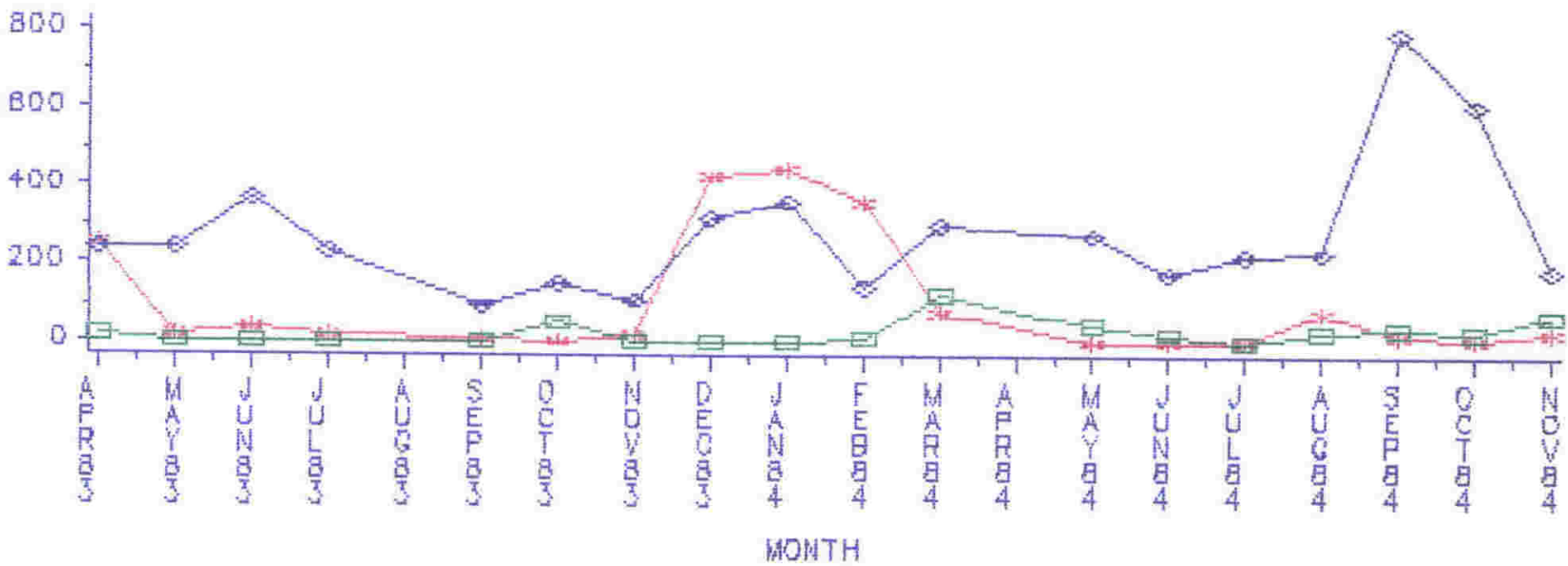


This could be due to the occasional pumping of fuel from tank 2 into the frigate and its replenishment from the tank cleaning vessel (TCV) may have contributed to the fluctuation of the number of fungi.

Table 3.8 lists the fungi isolated using the Kramer Collin's airsampler, and indicates their tolerance to creosote $(0.1 \%)$. Fusarium sp., Ulocladium sp., Aspergillus spp., Chaetomium globosum, Botrytis sp., Mucor sp., Rhizopus, Cladosporium cladosporioides and Aureobasidium sp. grew on culture plates exposed in the storage tanks indicating that they were air borne. Alternaria sp. occurred in abundance on filters through which fuel had been passed and also on the air spora plates with and without creosote. This fungus was only infrequently isolated from the fuels and it could be suppressed by other dominant fungi in fuel.

The fungi isolated from the soils surrounding the tanks are listed in table 2.1. C. resinae was isolated from soils surrounding the tanks. Most of the Penicillium spp. occurring in the fuel were isolated from the air spora plates but rarely from the soil. Among the different Penicillium spp. isolated from the soil by serial dilution and direct plating of the soil on selective media, $P$. expansum was the only species isolated from both the sources. $P$. variotii was not isolated from soil. All the species of Trichoderma occurring in the fuel were isolated from the soil. 


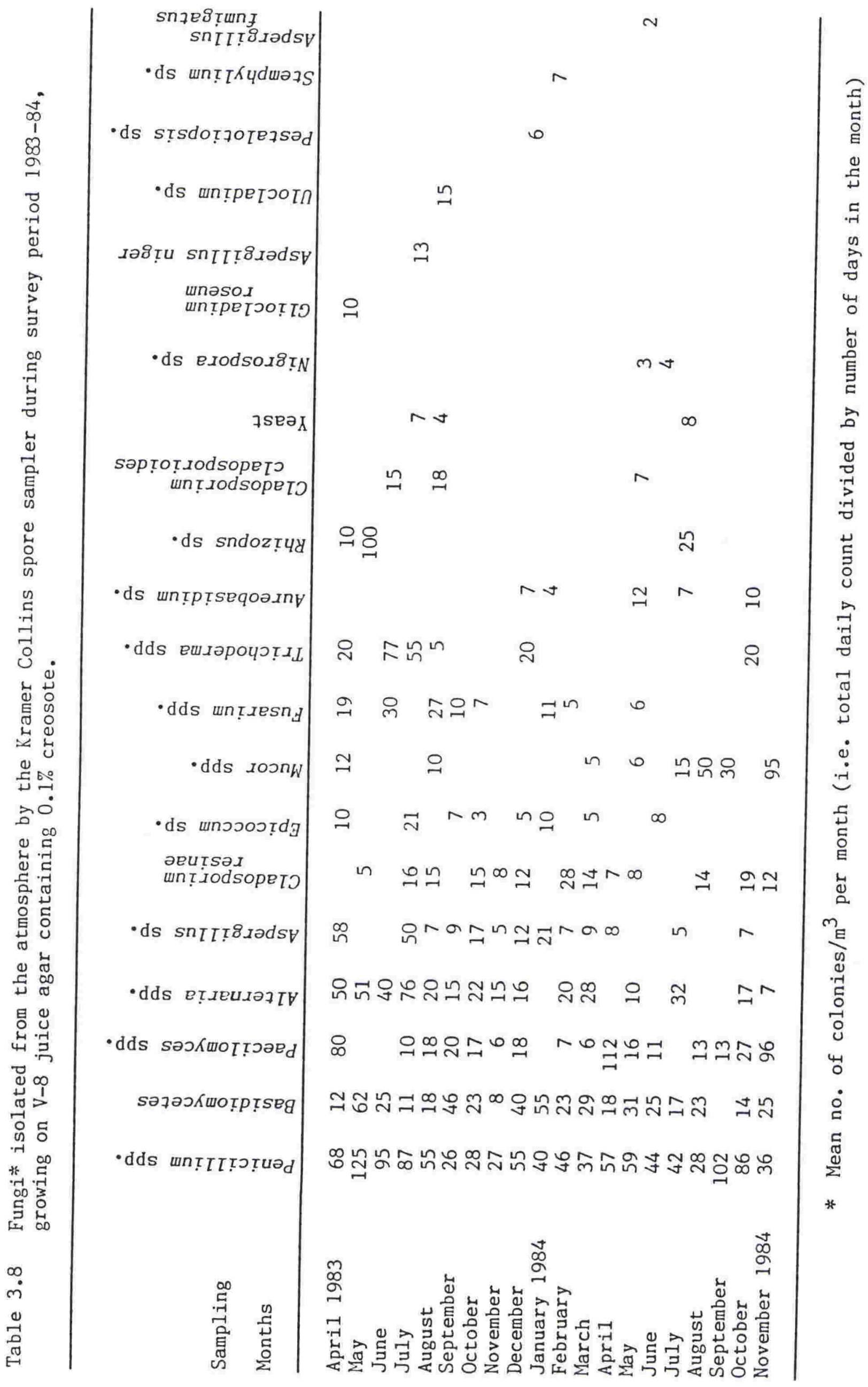


In this study of micro-organisms associated with the diesel fuel storage tanks system at Devonport, thirty-one fungal species were isolated during a twenty-four month period in 1982-1984. The most frequently isolated fungi were $C$. resinae, Penicillium spp. and Paecilomyces variotii. These could be classified as 'high activity' organisms capable of good growth in fuel. A similar range of organisms had been isolated from jet fuel (Sheridan and Soteros, 1974). Although C. resinae is a significant diesel contaminant, Penicillium spp. may be equally important as observed in jet fuel distribution systems (Soteros, 1973) and in diesel (Bruce, 1982). In both tanks, Penicillium spp. were found to be persistent and sometimes in greater number than $C$. resinae. Penicillium spp. also increased markedly with corresponding peak occurrence in the surrounding atmosphere. Most of the Penicillium spp. found in the fuel have been reported to be soil inhabitants (Pitt, 1979) but in this study, most of the species isolated from diesel fuel were present in the air spora.

The Paecilomyces spp., Penicillium spp., Fusarium spp., Alternaria spp., Trichoderma spp., Rhizopus sp., Mucor sp., Aureobasidium sp., Aspergillus spp., Epicoccum sp., and Botrytis sp. were known to be soil borne and air borne and have been reported to play a vital role in biodegrading different substrates (ZoBell, 1946; Miyoshi, 1895; Hendey, 1964; Lonsane, Singh and Baruah, 1975; Klausmeier and Andrews, 1981). They may have entered into the fuel from the atmosphere and remained as casual colonizers. The ability of some of these fungi to utilize diesel fuel is discussed in chapter 4.

If microbial fuel problems are directly related to the numbers of colony forming units able to be isolated from samples, then it is clear that both Penicillium and Paecilomyces must be included as well as $C$. resinae when considering causes and cures for problems due to micro-organisms in diesel fuel. It is not surprising that with time more micro-organisms will grow and become genetically adapted to the prevailing conditions in the fuel. 
In the main storage tanks there was a gradient in contamination levels of $C$. resinae. The highest concentration of CFU occurred at the bottom and 'very bottom' of the tanks. Data obtained from laboratory experiments conclusively showed a greater occurrence of C. resinae at the fuel/water interface and in water bottoms. It appears that the spores of $C$. resinae were suspended at the fuel-water interface. C. resinae spores are larger (3-7 x 2-4 $\mu \mathrm{m})$ than Penicillium spp. spores $(2.5-3 \mu \mathrm{m})$. C. resinae spores possibly have greater affinity for water and the settling out process may be more rapid. There was a higher incidence of microbiological contamination in tank 3 than in tank 2. The 'very bottom' of tank 3 constantly contained abundant water because all the water bottoms from the main storage tanks were usually drained into a separating tank. As the fuel partitions from the water in the separating tank, the fuel is pumped back into tank 3 and water to the sea, thereby unknowingly maintaining the contamination level in tank 3 .

The temperature and humidity of air entering the tanks through ventilation provide favourable conditions for the growth of the major fungi. These conditions would allow continuous condensation and accumulation of water particularly on the exposed tank wall and fuel surface. Diesel being viscous, water will settle out slowly and has a tendency to form and maintain a stable water-fuel interface.

Bacteria do not appear to be major diesel fuel contaminants. The detection of Pseudomonas sp., Micrococcus sp. and Bacillus sp. in the water bottom of the separating tank and the 'very bottom' of tank 3 , appears to show the necessity for free water for growth of bacteria in fuel tanks. Fass and Miller (1980) have suggested that bacteria die at a much faster rate than fungi in fuel. All the genera isolated in this study are known to contain hydrocarbon utilizers (Sohngen, 1913; ZoBel1, 1946). The aerobic bacteria in the separating tank have been reported to first lower the redox potential and this promotes suitable conditions for the growth of anaerobic sulphate reducers such as Desulfovibrio desulfuricans (Walter, 1971; Isenberg and Bennett, 1959). 
The $\mathrm{pH}$ of the environment is one of the controlling parameters for microbial growth and probably affects the stages of succession. It is known that bacteria and yeasts secrete low molecular weight fatty acids (Landsdown, 1965) which will subsequently lower the $\mathrm{pH}$ of the aqueous environment making it suitable for fungal colonization.

An alternative source of contamination which needs to be considered before any control measures for the storage tanks can be implemented is the fuel as supplied by the 'She11' and TCV. There is some degree of correspondence between the contamination detected in 'She11' and TCV as compared with tanks 2 and 3. Clearly any attempts at a general reduction must take this into account. The high level of microbial contamination in samples from TCV are of concern in that this could provide large contaminant inocula if this diesel is ever returned to vessels and storage tanks before being properly cleaned or filtered.

Techniques and sampling devices used for the isolation of bacteria have been in use for a considerable time (Sharpley, 1966; Hil1, Davies, Pritchard and Byron, 1967; Hil1, 1970) but there is a lack in the development of techniques for estimating viable fungal spores from hydrocarbon fuels. There has been little advancement over the last twenty years since Hazard (1963) developed the membrane filter technique. There is a need for the development of a better technique to isolate the increasing number of fuel contaminants. Among the methods used in this study, Hazzard's method proved to be the best. Hill (1970) described a rapid method for determining the level of contamination in aviation fuel condensate samples by testing the phosphatases, a group of enzymes widely distributed in microorganism. They split off phosphate from organic phosphate compounds which can readily be detected. The problem with such a chemical method is that it gives no indication about the identity of the potential contaminants.

Most methods used were found to have distinct disadvantages, some were unpredictable in that they did not give consistent and reproducible recoveries of viable organisms. 
Apart from the filtration method the other techniques have limitations which are reflected by the sample size used, and they are dependent upon the degree of contamination of the sample for them to be effective. Methods for the detection of viable organisms do not give accurate quantitative estimates of the number of organisms present in contaminated diesel fuel, as only viable organisms that grow on the culture medium are counted. Although counts have been expressed quantitatively they should be considered to be only qualitative. This is because a single fungal hypha may be fragmented during sampling and could give rise to a number of colonies from the fragments on culturing thereby indicating a count higher than the true one. Conversely, an aggregation of spores may be counted as one colony by giving rise to one mycelial mass.

The culture method of pouring the medium over the membrane inverted on the medium gave greatest numbers of the three major species. But placing the membrane on the medium with the right side up was understandably less valid. This method gave low recoveries of C. resinae. It may be that the hydrophobic nature of the membrane does not allow adequate nutrients to pass through for the spores to grow. The disadvantage of placing the membrane inverted on the medium is that the colonies growing on the surface of the medium, underneath the membrane are crowded, antagonism and other interactions between the organisms may have affected the number of viable counts. However using the modified Hazzard's method (1963) described in this work the above disadvantages are mainly overcome by pouring the medium over the membrane. In this way the spores are dispersed by the medium and the masking and suppression effects from over-crowding are reduced. This gave consistent and reproducible counts of viable organisms.

The pour and spread plate used for water bottom samples gave accurate and reliable counts when the sample of fuel was heavily contaminated. They were not satisfactory, when aliquots of fuel sample larger than $1 \mathrm{~cm}^{3}$ floated on the top of the medium. Growths submerged in fuel were difficult to see and the colonies appeared darker in colour. 
Time of analysis of the fuel samples was standardized to twentyfour hours despite some difference in the number of CFU of fungi recovered from zero to twenty-four hours. This difference was insignificant for this delay period. It was impossible to apply statistics to the colony numbers recovered due to the variable nature of the CFU which included spores and mycelial fragments, each having its own characteristic viability and multiplication rate. To have filtered two single, say $500 \mathrm{~cm}^{3}$ samples from each litre would have seriously underestimated contamination in most samples. While filtering twenty $50 \mathrm{~cm}^{3}$ samples could perhaps have resulted in a reasonably accurate estimate of viable CFU at that time. To test such numbers of replicates was impossible given the financial and human resources available. Thus the data obtained from the filters cultured from each sample will have some limitations because high numbers of colonies of a particular fungus may obscure some potential CFU of another fungus and the variation of this nature of the CFU makes statistical treatment irrelevant.

Although container type, analysis time, number of filters assayed were standardized, it was recognized that different individual fungi may have responded differently after removal from diesel fuel. Thus the results presented can only be discussed in rather general terms in order to attempt a better understanding of the source of and cure for microbial contamination. The aim throughout has been to obtain trends which may then be subjected to further laboratory and field testing under more defined conditions, hopefully giving some significant findings.

Direct observation of filters revealed a wide variety of fungal spore types in stored diesel fuel. Air spora studies suggest that many such as Alternaria, Epicoccum, Ulocladium, Trichoderma, Fusarium, C. cladosporioides and others could have entered the tanks from the surrounding air. But these fungi could not utilize the fuel, for they were highly selective as to the type of substrate that they would

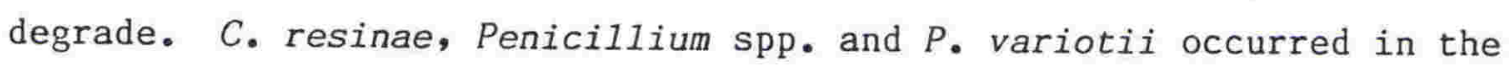
surrounding atmosphere near the tanks. However, CFU may arise from any of these potential contaminants as soon as there is water in the fuel. 
But the time lag in detecting the fungi in the 'very bottom' will depend upon the settling rate of the individual contaminant. Also the time lag between contamination and causing engine problems will depend on spores getting to the diesel/water interface, and subsequent laboratory experiments showed spores of all three major species can be detected as CFU on V-8 juice agar, immediately after inoculation at various periods of time.

Sharpley (1966) suggested a possible correlation between the seasonal variation of $C$. resinae in air and that in fuel. In the present study, Penicillium spp. were observed to occur in high levels in the atmosphere and a corresponding increase in CFU in the fuel, but this was less evident for $C$. resinae and Paecilomyces variotii. But air must still be recognised as a source of fuel contamination. Sheridan and Nelson (1971) and Sheridan (1974) monitored the atmosphere for $C$. resinae in Wellington and found similar autumn and summer peaks. But they did not correlate the seasonal incidence of C. resinae from the atmosphere to its incidence in fuel. It appears that contamination of fuel is a complex problem.

According to Reichart (1983) fungal spores are always present in the atmosphere and therefore will eventually find their way into fuel tanks but these are harmless unless water is present to provide the ideal habitat for growth. Once established the fungus produces water as a by product of growth, which is probably the reason for its rapid proliferation.

One obvious source of the air spora isolated near the tanks in this study was analysed by determining the soil microflora and it is clear that both $C$. resinae and a number of Penicillium spp. were abundant adjacent to the tanks. C. resinae was found to be widespread in soil in New Zealand (Sheridan and Knox, 1970; Sheridan, 1974). 
The possible sources and causes of high levels of microbial contamination in the storage tanks at Devonport may be summarised as follows:

- The major fuel-utilizing contaminants were C. resinae, Penicillium corylophilum and Paecilomyces variotii.

- Tank 3 had some continuous re-inoculation by the contaminants because of the regular return of fuel and water from the separation tank.

- Fuel as supplied is seldom (if ever) free of microbial contamination.

- Fungal spores from the air were a continuous source of inoculum and this was significant for Penicillium spp., compared with $C$. resinae and $P$. variotii.

- Any source of inoculum will only present a problem if the fungi can grow and this requires a free aqueous phase which is commonly present. 


\subsection{Growth studies}

\subsubsection{Single species culture}

A) Isolates from this study:

Figure 4.1 shows the growth of diesel isolates of $C$. resinae, $P$. variotii and $P$. corylophilum after 6 weeks in medical flats containing equal volumes of Bushnell-Haas (BH) and diesel in the laboratory. These fungi formed mycelial mats about $5 \mathrm{~mm}$ thick from an inoculum of $5 \times 10^{6}$ spores $/ \mathrm{cm}^{3}$ when grown at $25^{\circ} \mathrm{C}$. Sporulation could be perceived after one week with spores being in the diesel phase. Mycelial debris were present in the aqueous phase from 4 weeks onwards.

Table $4.1 \mathrm{~A}$ and figure 4.2 show that all isolates of $C$. resinae, $P$. corylophilum and $P$. variotii grew well in Bushnell-Haas/diesel phases and showed a high degree of viability in subsequent culture on V-8 juice agar (table 4.1B). Figure 4.3 shows the growth of these fungi in different aqueous phases apart from in BH/fuel phases. On seawater/diesel phases only $P$. corylophilum grew well. In the tapwater/diesel phases $P$. variotii however appeared to survive to a higher degree. It did not produce as many viable spores in the distilled water/diesel phases. In diesel alone none of the fungi tested grew significantly although spores of all fungi except for P. corylophilum remained viable. The $\mathrm{pH}$ of the aqueous phases remained unchanged. There was a time lag period of 2-3 days for $C$. resinae and $P$. variotii.

B) Isolates from other sources:

Australian isolates of $C$. resinae and the NZ diesel isolate showed similar growth on the BH/diesel phases producing thick dark mycelial mats. The white form of $C$. resinae from Australia produced only a thin white mycelial mat. The dry weight yields of fungal material after 6 weeks growth in the $100 \mathrm{~cm}^{3}$ culture were as follows: 
Figure 4.1. Growth of Cladosporium resinae, Paecilomyces variotii and Penicillium corylophilum (ex diesel fuel) in Bushne11-Haas/diesel fuel interface after six weeks. Note:- Mycelial debris in the aqueous phase.

Figure 4.3. Growth of various types on aqueous/diesel fuel phases a expressed in table 4.1A.

Left:- Some spore germination, very little growth (+) Centre:- Spore layer (inoculum) unchanged (0). Right:- Very thin colourless mycelial mat (++). 

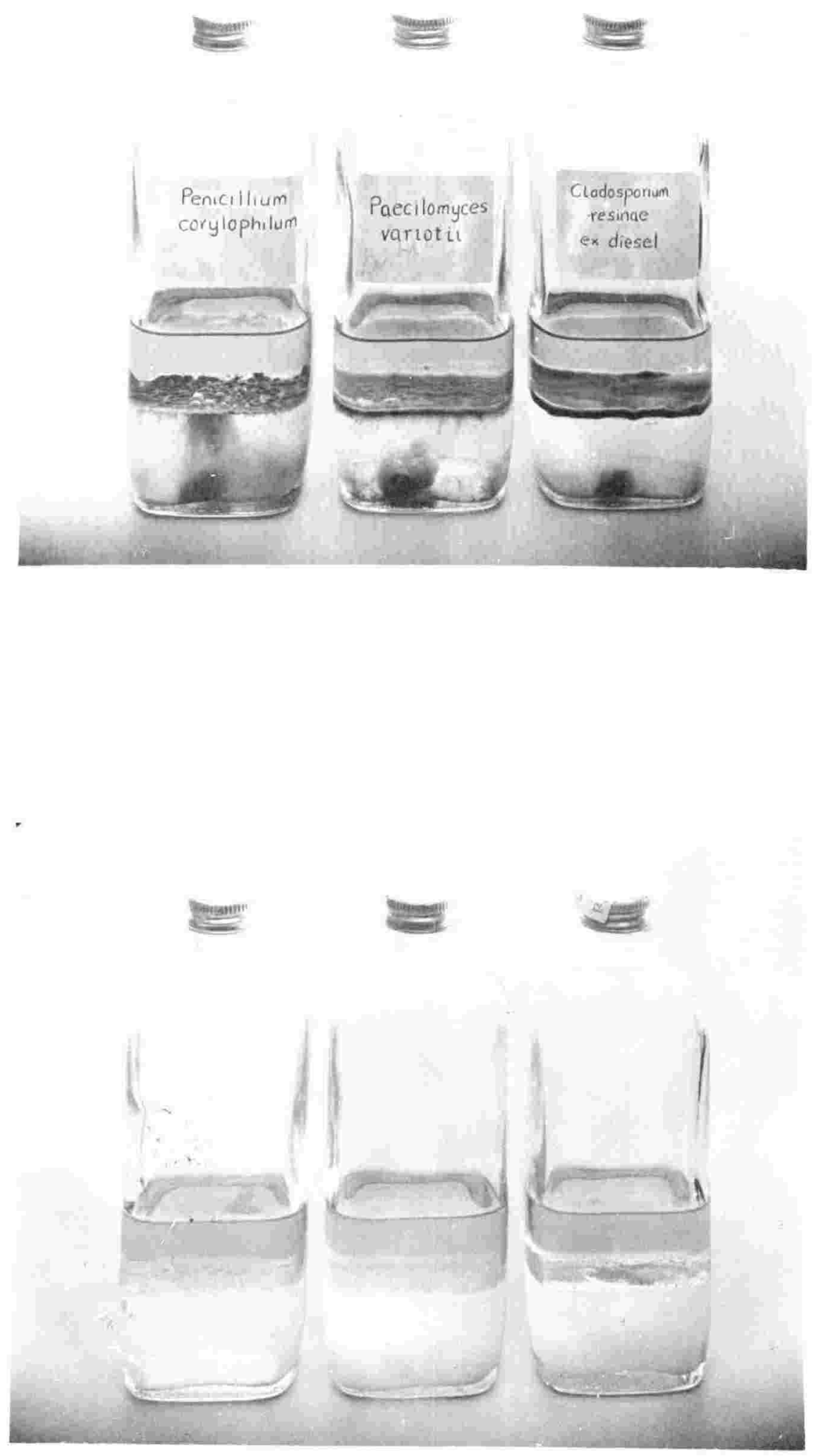
Figure 4.2. Growth* of fungal isolates on various aqueous/diese1 fuel phases $(1: 1)$.

Type $1-C$. resinae ex diesel fuel.

2 - C. resinae ex jet fuel.

3 - C. resinae ex Air.

4 - C. resinae ex soil.

5 - Penicillium corylophilum.

6 - Paecilomyces variotii.

* Growth is expressed as the mean dry weight of fungus recovered by filtration from duplicate $100 \mathrm{~cm}^{3}$ cultures inoculated with $5 \times 10^{6}$ spores $/ \mathrm{cm}^{3}$. 


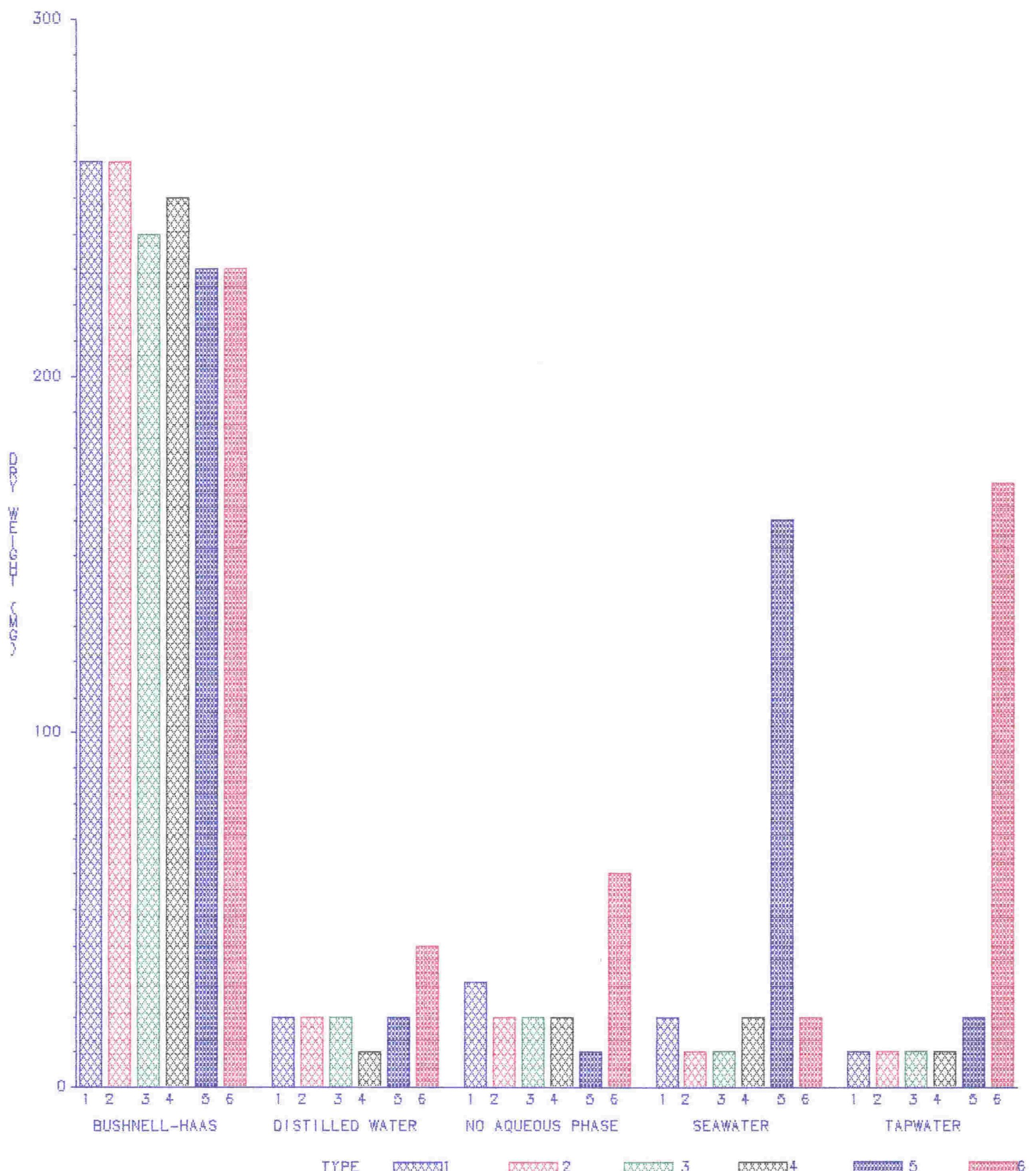


Table 4.1A Growth $^{*}$ of principal microbial contaminants in the laboratory on various aqueous/diesel fuel phases.

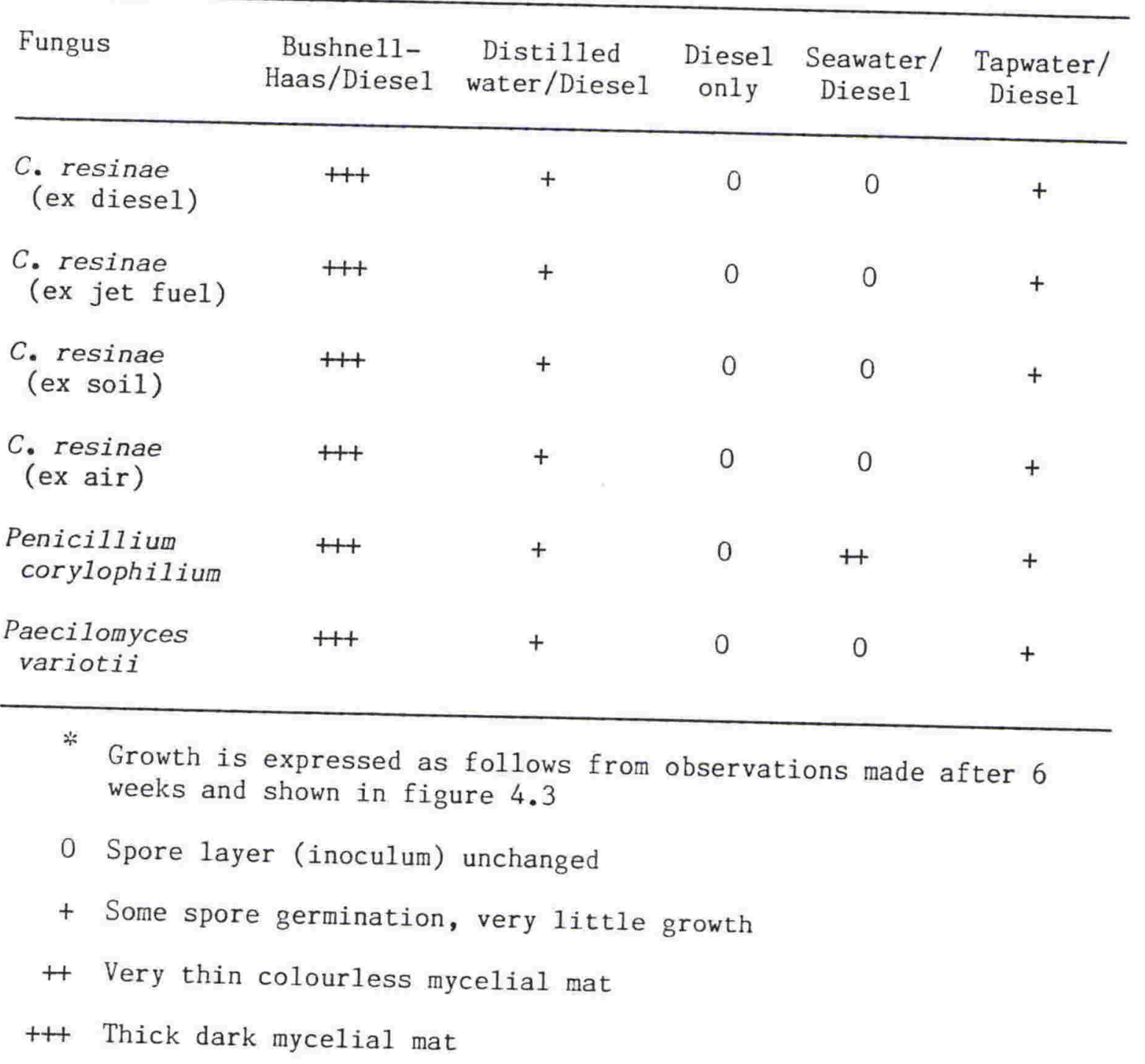


Table 4.1B Viability ${ }^{+}$of fungi/spores isolated after 6 weeks and grown on $\mathrm{V}-8$ juice agar.

\begin{tabular}{|c|c|c|c|c|c|}
\hline Fungus & $\begin{array}{l}\text { Bushnel1- } \\
\text { Haas/Diesel }\end{array}$ & $\begin{array}{l}\text { Distilled } \\
\text { water/Diese1 }\end{array}$ & $\begin{array}{l}\text { Diesel } \\
\text { only }\end{array}$ & $\begin{array}{l}\text { Seawater/ } \\
\text { Diese1 }\end{array}$ & $\begin{array}{l}\text { Tapwater/ } \\
\text { Diese1 }\end{array}$ \\
\hline $\begin{array}{l}\text { C. } \text { resinae } \\
(\text { ex diesel) }\end{array}$ & +++ & + & + & 0 & + \\
\hline $\begin{array}{l}\text { C. resinae } \\
(\mathrm{ex} \text { jet fuel) }\end{array}$ & ++ & + & + & 0 & + \\
\hline $\begin{array}{r}\text { C. resinae } \\
(\text { ex soil) }\end{array}$ & ++ & + & + & 0 & ++ \\
\hline $\begin{array}{l}\text { C. resinae } \\
(\mathrm{ex} \text { air })\end{array}$ & $H+$ & + & + & 0 & + \\
\hline $\begin{array}{l}\text { Penicillium } \\
\text { corylophilum }\end{array}$ & ++ & + & 0 & $H$ & + \\
\hline $\begin{array}{l}\text { Paecilomyces } \\
\text { variotii }\end{array}$ & +++ & + & + & 0 & +++ \\
\hline
\end{tabular}

\footnotetext{
+ Viability of fungi, colony frequency, 5 days after pour plate
inoculation.

0 no colonies

+10 colonies $/ \mathrm{cm}^{3}$ or less

+10-50 colonies $/ \mathrm{cm}^{3}$

$++>50$ colonies $/ \mathrm{cm}^{3}$
} 


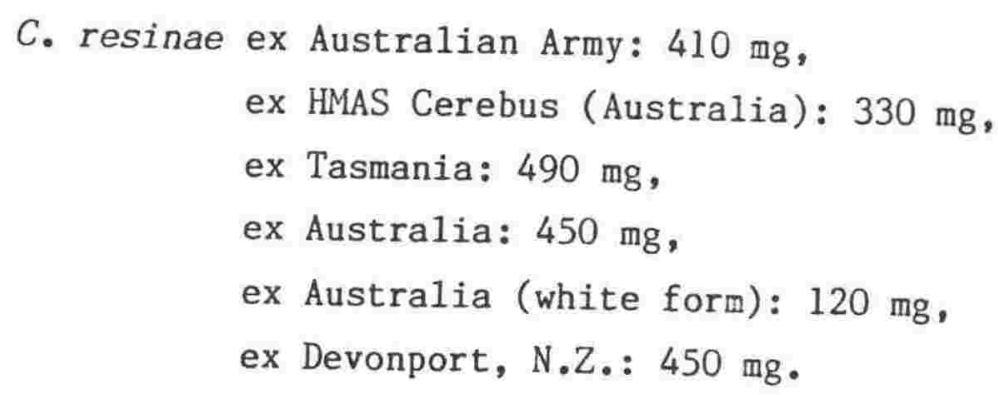

Table 4.2 compares the growth of the seven species of Penicillium isolated from diesel fuel with that of $P$. variotii and various $C$. resinae isolates on both Bushnell-Haas/diesel and $\mathrm{BH} / \mathrm{jet}$ fuel. Only $P$. corylophilum grew well compared to the other Penicillium spp., $P$. expansum and $P$. spinulosum showed little growth. Spores of all Penicillium spp. except $P$. digitatum appeared to retain full viability after 6 weeks in the BH/diesel fuel phases.

C) Soil isolates:

Among the soil isolates obtained from around the cliff tanks, only Penicillium nigricans and $P$. janthinellum showed any signs of growth on $\mathrm{BH} /$ diesel fuel phases. This growth was very limited producing $80 \mathrm{mg}$ and $50 \mathrm{mg}$ dry weight respectively after six weeks. No growth occurred in BH without fuel or in diesel fuel alone.

\subsubsection{Growth in mixed cultures}

i) When $P$. corylophilum and $C$. resinae were grown as a mixed culture in the Bushnell-Haas/diesel phases, a thick dark mycelial mat was produced after 6 weeks. $P$. corylophilum spores were not visible in samples of the well shaken cultures and on plating out on $\mathrm{V}-8$ juice agar, only colonies of C. resinae were produced. By contrast in seawater/diesel growth medium, no visible growth had occurred after 6 weeks. Microscopic observation indicated that $P$. corylophilum spores germinated but not $C$. resinae spores. When the seawater/diesel fuel sample was plated onto V-8 juice agar only $P$. corylophilum colonies were obtained. Little growth occurred in tapwater/diesel phases but spores of both fungi retained viability for 6 weeks after mixed spores were inoculated. 
Table 4.2 Growth and survival of Penicillium ${ }^{*}$ spp., C. resinaet and $P$. variotii on Bushnell-Haas (BH) plus diesel or jet fuel.

Fungi

Growth $^{1}$ after 6 weeks
in $\mathrm{BH} /$ diesel or jet fuel

Funga1

viability ${ }^{2} \mathrm{x}$

$$
\begin{aligned}
& \text { P. echinulatum } \\
& \text { P. chrysogenum } \\
& \text { P. frequentans } \\
& \text { P. spinulosum } \\
& \text { P. corylophilum } \\
& \text { P. expansum } \\
& \text { P. digitatum } \\
& \text { C. resinae (ex jet fuel) } \\
& \text { C. resinae } \\
& \text { C. resinae } \\
& \text { C. resinae } \\
& \text { P. variotii }
\end{aligned}
$$

$$
\pm
$$$$
\pm
$$$$
\pm
$$$$
+
$$$$
++
$$$$
+
$$$$
\pm
$$$$
\text { H+ }
$$$$
++
$$$$
++
$$$$
\text { H+ }
$$$$
\text { H+ }
$$

市

The seven Penicillium spp were all isolated from diesel fuel in this study.

+ C. resinae isolated from diesel fuel, soil and air in this study and one previously isolated from jet fuel were grown.

\# $P$. variotii isolated from diesel fuel in this study.

1 Identical growth responses were obtained regardless of hydrocarbon type.

\pm Very thin spore mat

+ Thick spore mat, very thin colourless mycelial mat.

+++ Thick dark mycelial mat.

2 Identical growth patterns were observed regardless of hydrocarbon type.

$x$ Viability after 6 weeks growth was determined by the pour plate method using $5 \mathrm{~cm}^{3}$ sample from shaken cultures and is expressed simply as a proportion of Petri-dish covered by fungal growth after 5 days. 
In diesel fuel without aqueous phase, no growth occurred of either fungus but spores of $C$. resinae retained some viability.

ii) The effect of metabolic changes brought about by growth of C. resinae on subsequent growth of Penicillium and Paecilomyces is shown in table 4.3. P. corylophilum and $P$. variotii grew well following growth of $C$. resinae on $\mathrm{BH} /$ diesel producing just as much dry weight after the 6 weeks growth period as had been produced on fresh $\mathrm{BH} /$ diesel phases. No significantly increased growth was observed on distilled water/diesel and diesel alone. Only $P$. corylophilum grew on seawater/diesel fuel phases. Both $P$. corylophilum and $P$. variotii produced little growth on tapwater. There was only a slight shift in the $\mathrm{pH}(8.3-8.1)$ and $(7-6.8)$ in the seawater/diese1 fuel and $\mathrm{BH} / \mathrm{diese} 1$ fuel

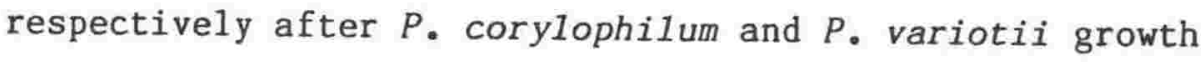
period. No significant change in $\mathrm{pH}$ was observed after C. resinae was grown in above mentioned media. The other aqueous phases remained neutral ( $\mathrm{pH} 7)$. As shown in table $4.4 \mathrm{~A}$, in a more extensive study of the effect of the presence of one fungus upon the growth of another, little growth was observed in the absence of diesel fuel. All cultures containing $P$. corylophilum alone or as a mixed inoculum showed growth after 6 weeks in seawater.

Survival of ungerminated and germinated spores in aqueous phases alone and aqueous/diesel fuel phases is shown by the data in table 4.4B. Candida did not survive on tapwater, alone or as a mixed spore inoculum; while its survival in $\mathrm{BH}$ was affected by the accompanying fungus. Germinating $C$. resinae, $P$. corylophilum and Paecilomyces variotii spores appeared to make Candida spores non-viable after 6 weeks. Candida retained some viability after 6 weeks in seawater. Again this was reduced by the presence of other fungi and was eliminated by C. resinae, Penicillium corylophilum, P. expansum and Paecilomyces variotii. In tapwater $C$. resinae suppressed the viability of $P$. expansum. 


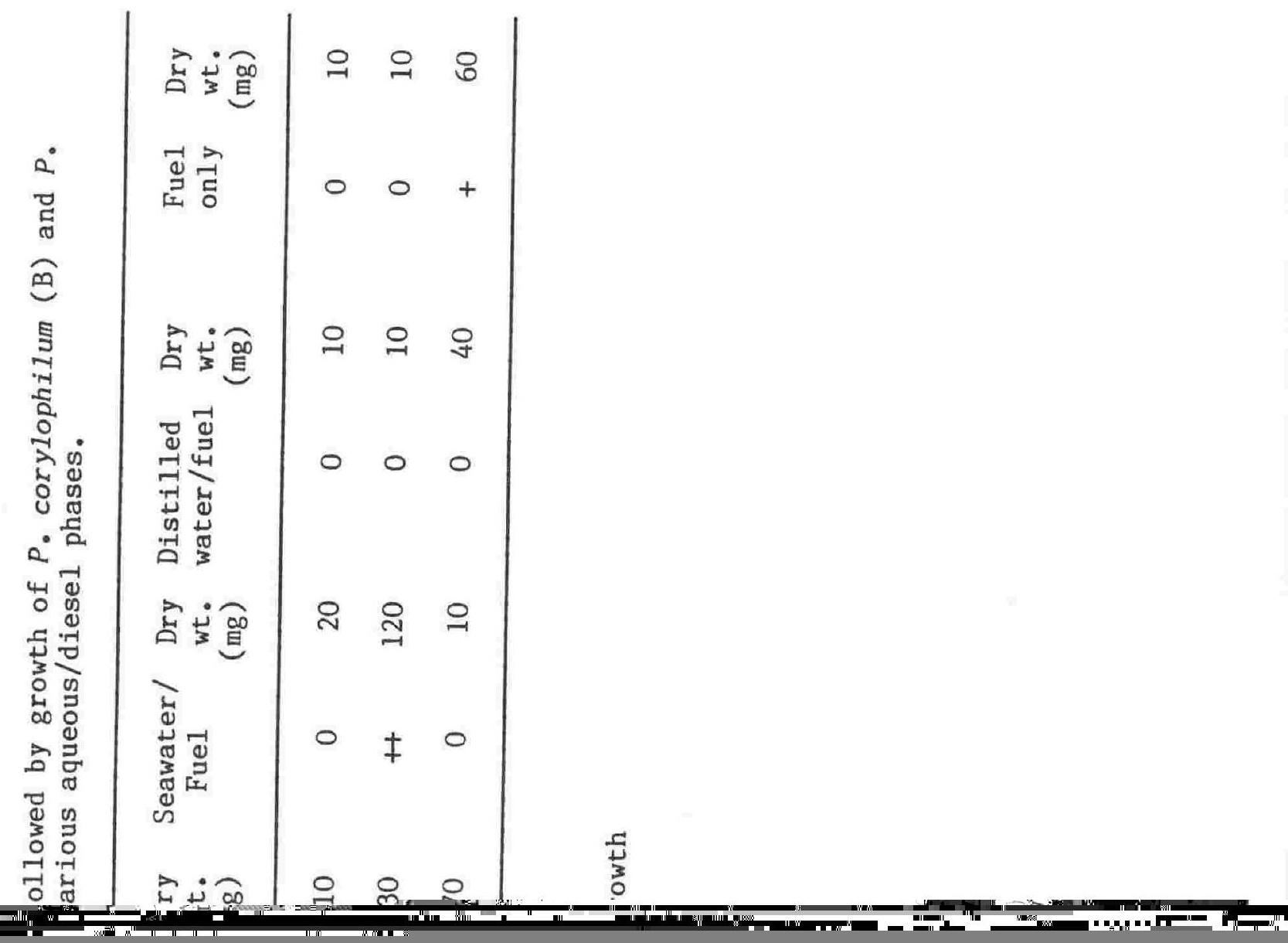




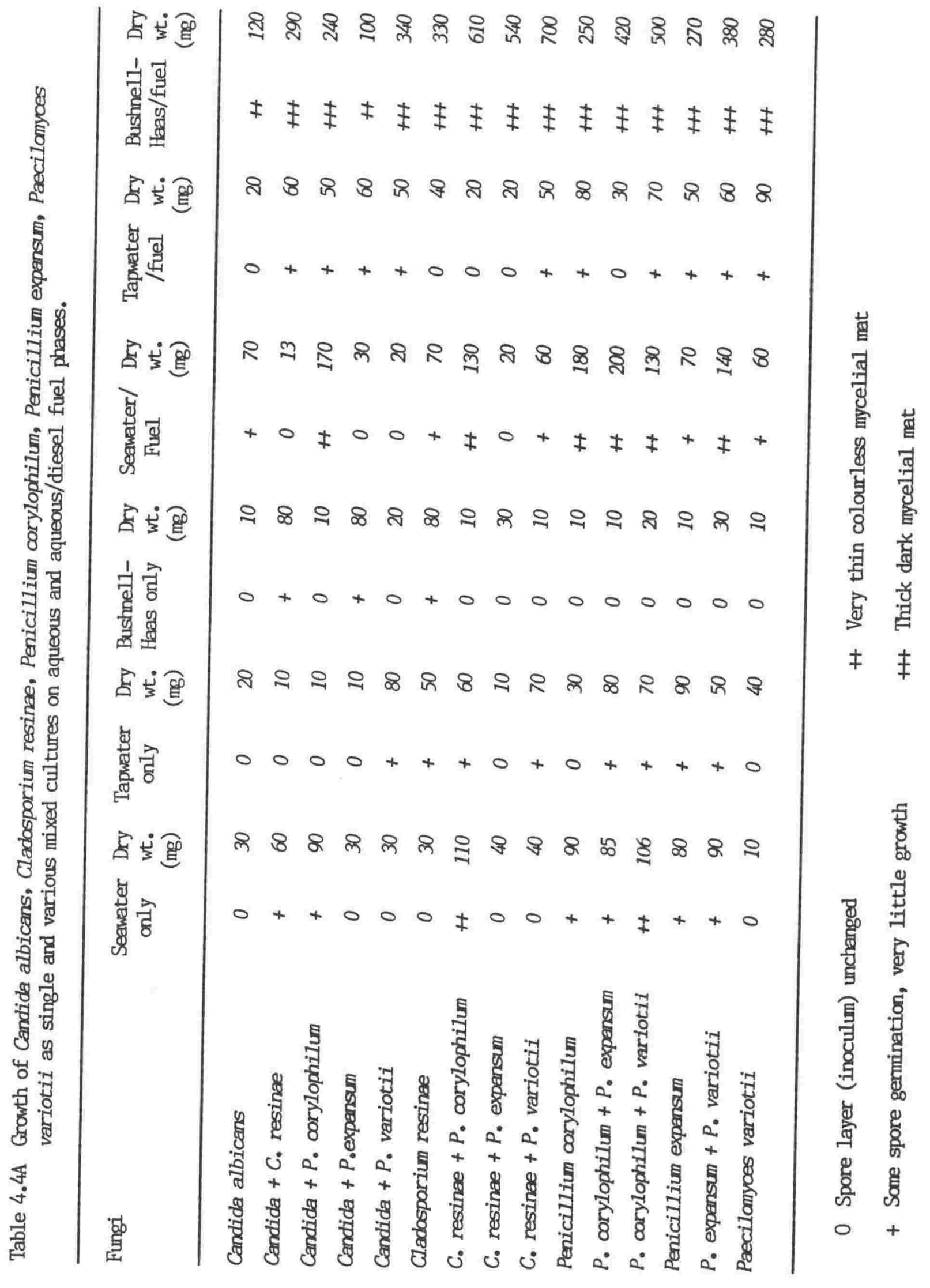


* Viability is expressed as the number of colonies observed 5 days after plating $5 \mathrm{~cm}^{3}$ sample on $\mathrm{V}-8$ juice agar.

0 Absence of colonies

$<50$ less than $50 \%$ viable spores

$>50$ more than $50 \%$ viable spores 


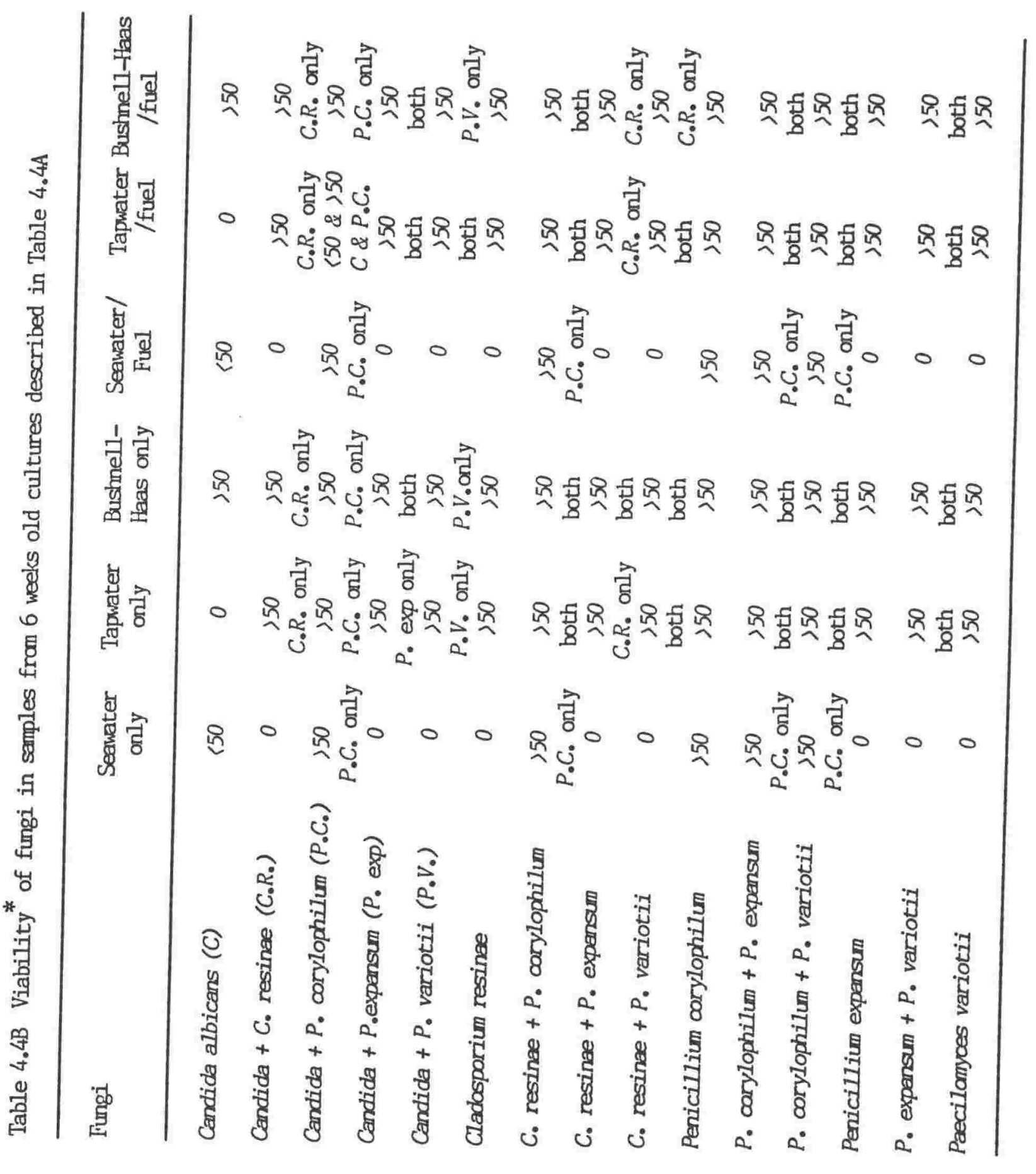


In tapwater/diesel fuel phases, Candida showed least evidence of growth. In seawater/diesel, $P$. corylophilum showed some growth and this appeared unaffected by the presence of other fungi nor was the viability of $P$. corylophilum reduced after 6 weeks in mixed culture. Candida retained viability after 6 weeks in seawater/diesel but the viability was completely suppressed by $P$. corylophilum.

As already shown BH/diesel phases supported considerable growth of fungi, even of Candida. The total growth of Candida and $P$. expansum was considerably less than that of either fungus alone but viability of either fungus appeared to be unaffected when grown alone or as a mixed inoculum. When $C$. resinae, $P$. corylophilum and $P$. variotii were growing profusely in the presence of Candida, Candida could no longer be recovered after 6 weeks growth in BH/diesel. Similarly, C. resinae appeared to eliminate viable spores of $P$. expansum and $P$. variotii after 6 weeks of growth.

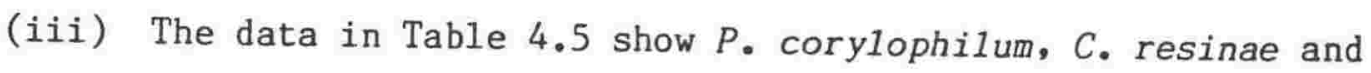
$P$. variotii produced thick dark mycelial mats in $\mathrm{BH} /$ diesel phases. P. corylophilum produced little growth in seawater/diesel and no significant change in growth pattern was observed in other aqueous phases.

After the first 6 weeks, when $C$. resinae, $P_{\text {. variotii and }}$ $P$. corylophilum were inoculated in $P$. corylophilum, $C$. resinae and $P$. variotii grown media respectively, they continued to form dark mycelial mats in the $\mathrm{BH} / \mathrm{fuel}$. The media did not seem to have any limiting effect on the growth of the second batch of fungi. But in the seawater/fuel, $C$. resinae formed a very thin colourless mycelial mat after $P$. corylophilum had grown on the same media. When $C$. resinae was grown initially in seawater/fuel, it produced only a spore layer and the dry weight was insignificant. $P$. corylophilum grown media may have undergone some metabolic changes enhancing $C$. resinae survival in seawater/fuel and seawater only. By contrast there was a change in $\mathrm{pH} 8.3$ to 8 . 


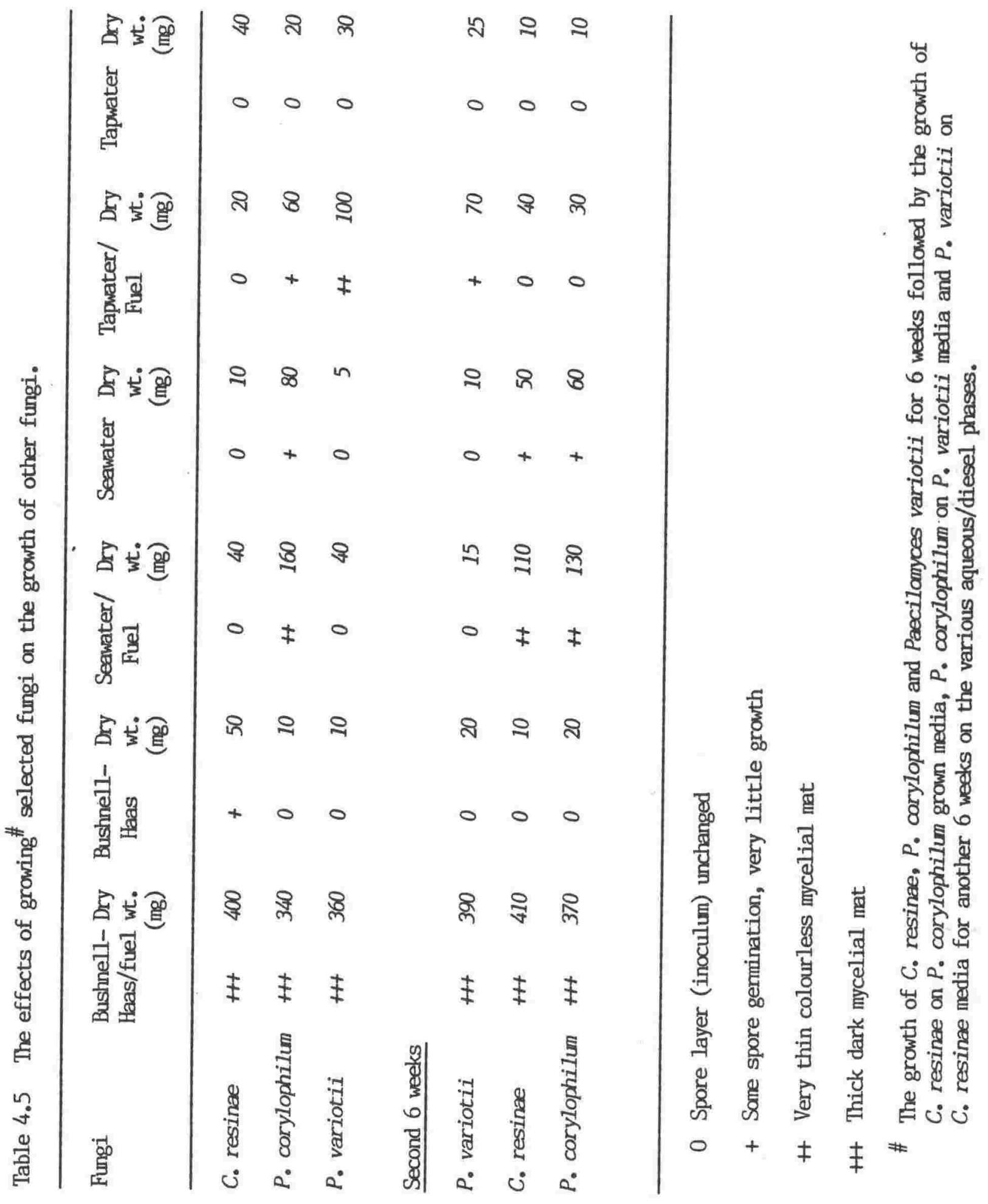


P. corylophilum grew singly and regardless of the other type of fungi grown in its media.

\subsection{Creosote tolerance studies}

The effects of creosote on the growth of fungi is shown by the data in figures $4.4 \mathrm{~A}, \mathrm{~B}, \mathrm{C}, \mathrm{D}$ and $\mathrm{E}$. The $C$. resinae isolates (ex diesel, jet fuel, soil and air) and $P$. corylophilum and $P$. variotii (ex diese1) grew on V-8 juice agar in the presence of creosote concentration up to $1 \%$. No growth occurred at concentrations of $2 \%$ creosote. C. resinae f. albidum (ex Australia) also tolerated 1\% creosote but the N.Z. isolate of this form appeared slightly less tolerant. $P_{\text {. expansum, }} P_{\text {. digitatum, }} P_{\text {. frequentans, }} P_{\text {. chrysogenum, }}$ $P$. echinulatum and Cladosporium cladosporioides from diesel were able to grow and tolerate up to $0.5 \%$ creosote. Alternaria alternata ex air, ex diesel and ex jet fuel tolerated up to $0.3 \%$ creosote. Except for $P$. brevicompactum and Trichoderma hamatum, the other fungi isolated from soil and other sources in this study showed growth in the presence of creosote but only up to a concentration of $0.3 \%$.

\subsection{Effects of shaking and transport on spore viability}

Spores of C. resinae, Penicillium corylophilum and Paecilomyces variotii appeared to be completely unaffected by being shaken in diesel fuel, by hand for up to 30 minutes, or by mechanical agitation for up to 8 hours, whether as single or mixed inocula when viability was tested by the millipore filtration method.

The effect of carrying inoculated diesel or jet fuel samples by car over various distances (300-500 km) during 2-3 days, equally was shown to have no influence on the viability of $C$. resinae isolated from either diesel or jet fuel. $100 \%$ spore germination recovery was obtained from both the diesel and jet fuel. 
Figure 4.4. Growth of the various isolates on V-8 juice agar containing different concentrations of creosote (after 14 days at $25^{\circ} \mathrm{C}$ ).

A. Soil Isolates

Type 1. C. resinae. 2. Trichoderma harzianum.

3. Penicillium aculeatum. 4. P. funiculosum.

5. P. janthinellum. 6. P. 1oliense. 7. P. nigricans.

8. P. verrucosum. 9. P. velutinum.

B. Diese1 Isolates (Page 78)

Type 1. C. resinae f. albidum. 2. C. resinae.

3. Paecilomyces variotii. 4. Penicillium corylophilum.

5. P. chrysogenum. 6. P. digitatum. 7. P. expansum.

8. P. echinulatum. 9. Alternaria alternata.

10. Penicillium frequentans. 11. P. spinulosum.

12. Cladosporium cladosporioides.

C. Jet Fuel Isolates (Page 79)

Type 1. C. resinae f. resinae. 2. C. resinae $f$. avellaneum. 3. C. sphaerospermum. 4. Alternaria al ternata.

D. Australian Diesel Fuel Isolates (Page 80)

Type 1. C. resinae ex albidum. 2. C. resinae ex Australia. 3. C. resinae ex Army . 4. C. resinae ex Cook Island. 5. C. resinae ex HMAS Cerebus. 6. C. resinae ex Tasmania.

E. Others (Page 81)

Type 1. Cladosporium cladosporioides ex Air.

2. C. resinae $f$. resinae ex feather.

3. C. cladosporioides ex tomato.

4. Alternaria alternata. 


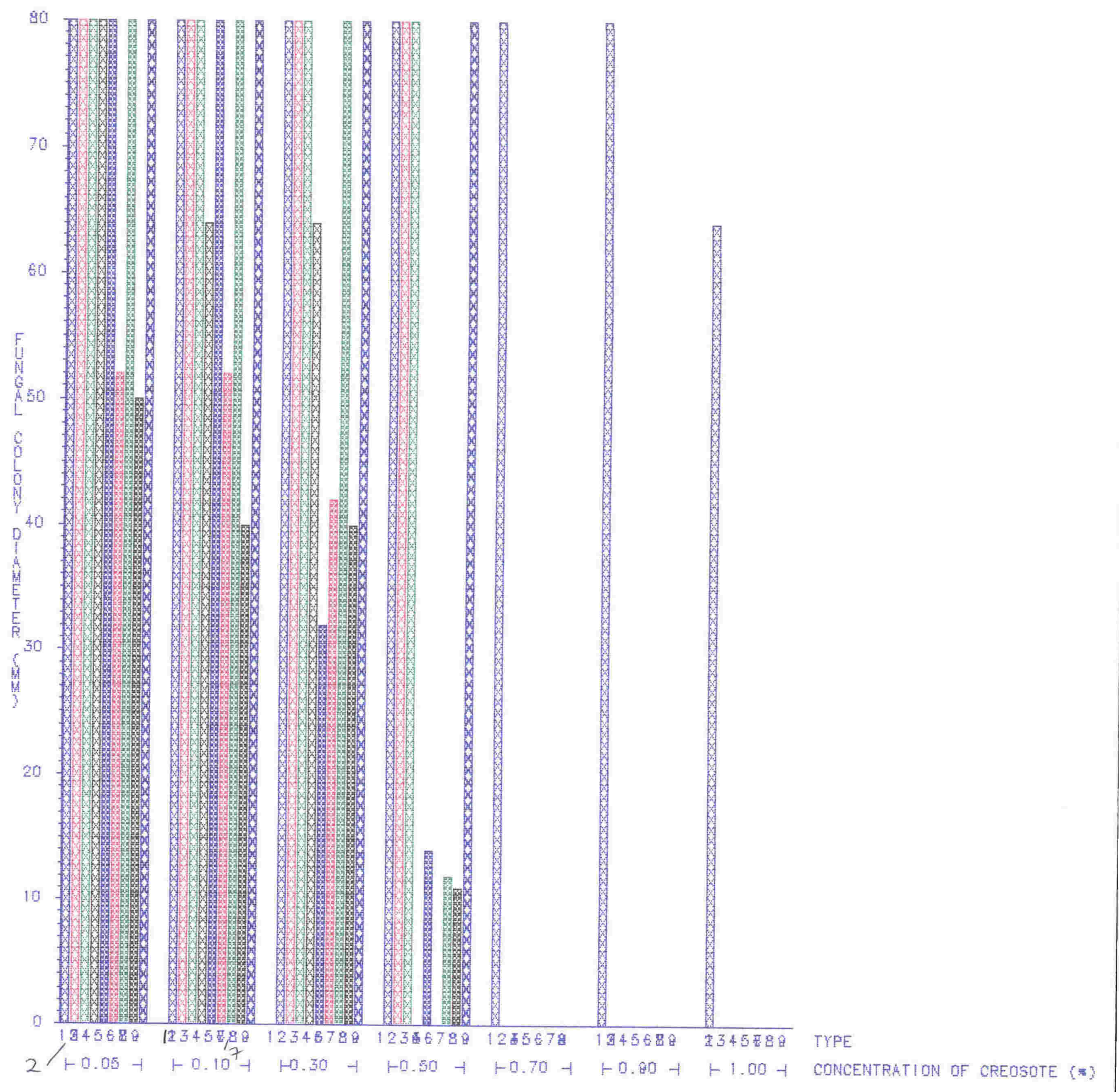

TYPE एWWO 1
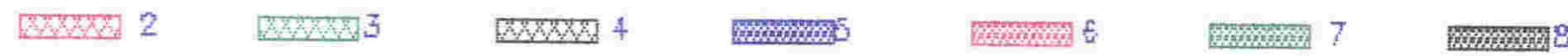

WWOW: 9 


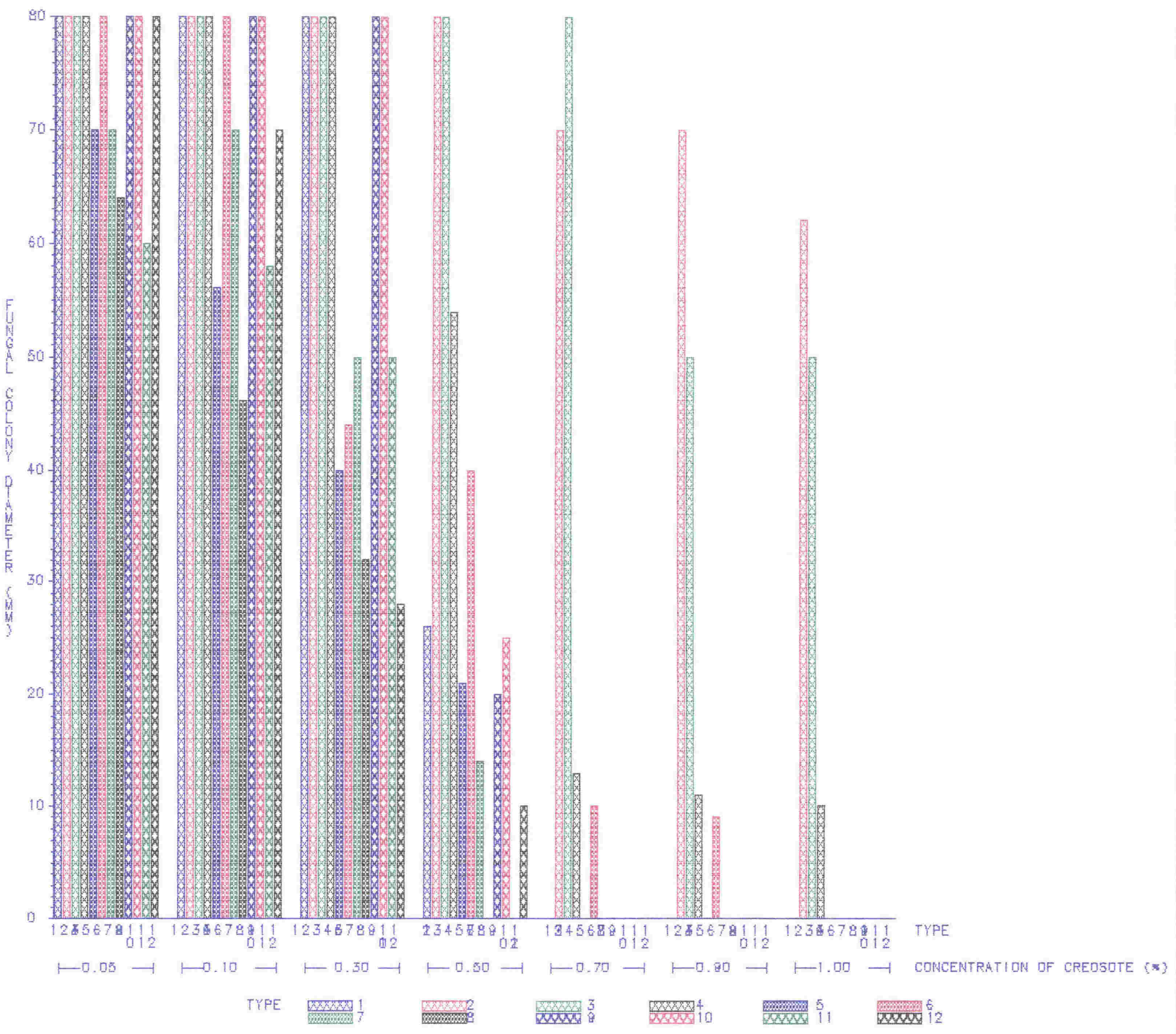




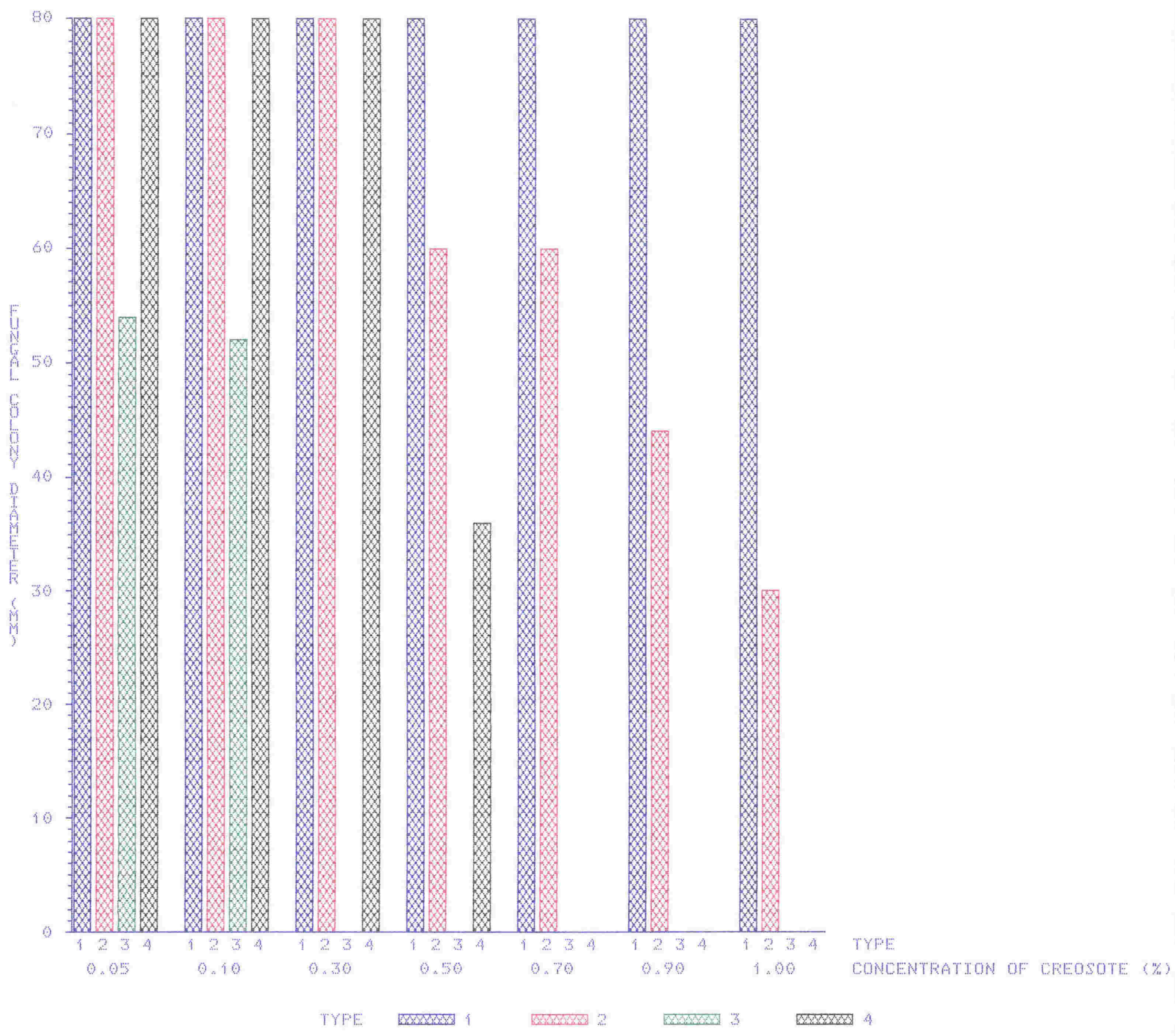




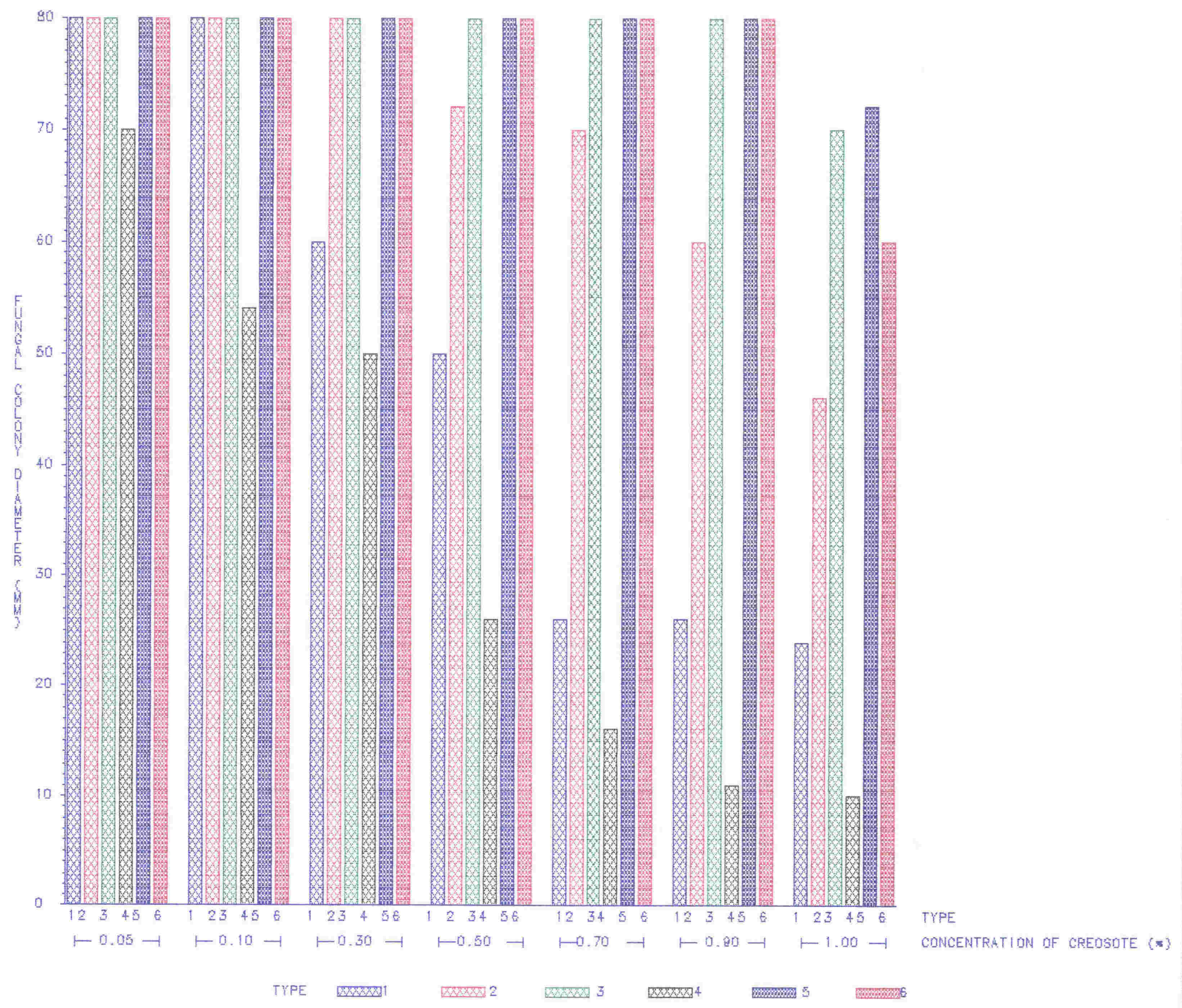




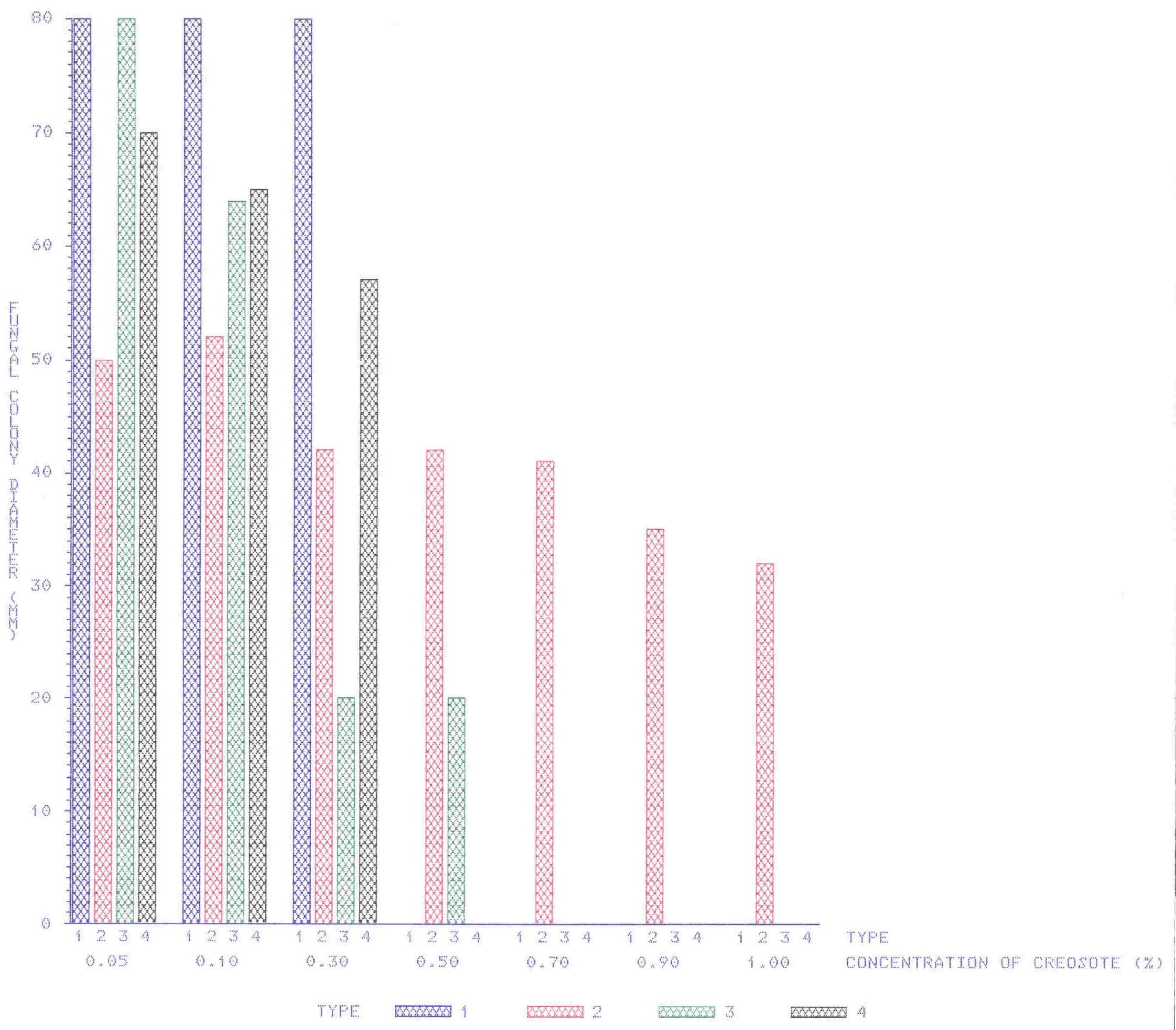




\subsection{Morphology and ultrastructure studies}

Five fungi $C$. resinae ex jet, ex diesel, Paecilomyces variotii, Penicillium corylophilum and Cladosporium cladosporioides were studied using light and scanning electron microscopy (SEM) in order to see if any surface features could be identified as being common to those able to use hydrocarbon. Representative photographs of conidiophores and conidia of these fungi in the light and scanning electron microscopy are represented in figures $4.5 a, b, c, d$ and $e$ and $4.6 a, b, c, d$ and e.

The conidiophores and conidia of $C$. cladosporioides were roughwalled, but for the other fungi, they appeared smooth-walled in light microscopy. In SEM, the conidiophore, ramoconidia and blastospores of C. resinae (ex jet fuel) appeared smooth, whereas in C. resinae ex diesel, the above structures appeared 'crinkled'. Both forms usually had 2-3 projections on the ramoconidia. It was also evident in the SEM micrographs that the conidia of $C$. resinae were not separated from each other by prominent scars, hence the name Hormoconis resinae (Lindau) Von Arx and de Vries is acceptable.However C. cladosporioides conidia had scars and the conidiophore was less ornamented than the conidia. The sporulating structures of $P$. variotii were smooth. The phialides were drawn out to produce the conidia in chains.

In contrast to the light micrographs, the stipe of $P$. corylophilum appeared warted but the phialides and conidia were smooth-walled. No single characteristic can clearly be associated with those fungi capable of utilizing diesel fuel.

Techniques for fixing and staining material for ultrastructural characteristics using transmission electron microscopy of the sections varied considerably in their effectiveness. Figures 4.7-1, 2 and 3 show the appearance of sections of conidia of $C$. resinae (ex diesel) which have been prepared using a range of techniques. Many of these techniques show inadequate details in the conidia ultrastructure.

In methods 1-4, stated in Table 2.4, the cell wall appeared disintegrated and the cytoplasm and cellular components were dark (figure 4.7-1). 
Figure 4.5. Light micrographs of conidiophores and conidia.

a. Cladosporium resinae $f$, avellaneum.

b. Cladosporium resinae $f$. avellaneum ex jet fuel.

c. Cladosporium cladosporioides ex soil.

d. Paecilomyces variotii ex diesel fuel.

e. Penicillium corylophilum ex diesel fuel. 

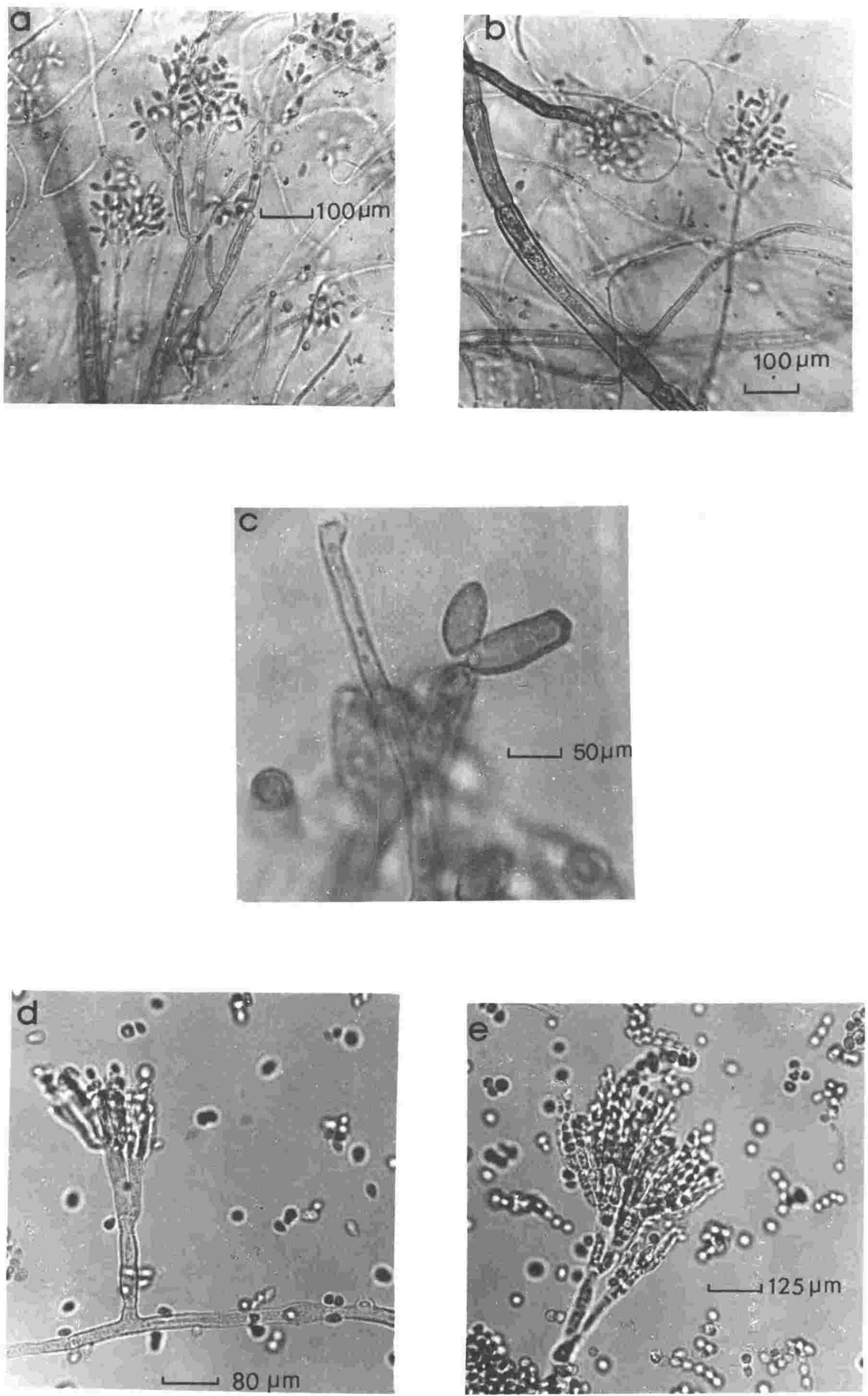
Figure 4.6. Scanning electron micrographs (SEM) of conidiophores and conidia.
a. Cladosporium resinae $f$. avellaneum ex diesel fuel.
b. Cladosporium resinae $f$, avellaneum ex jet fuel.
c. Cladosporium cladosporioides ex soil.
d. Paecilomyces variotii ex diesel fuel.
e. Penicillium corylophilum ex diesel fuel. 

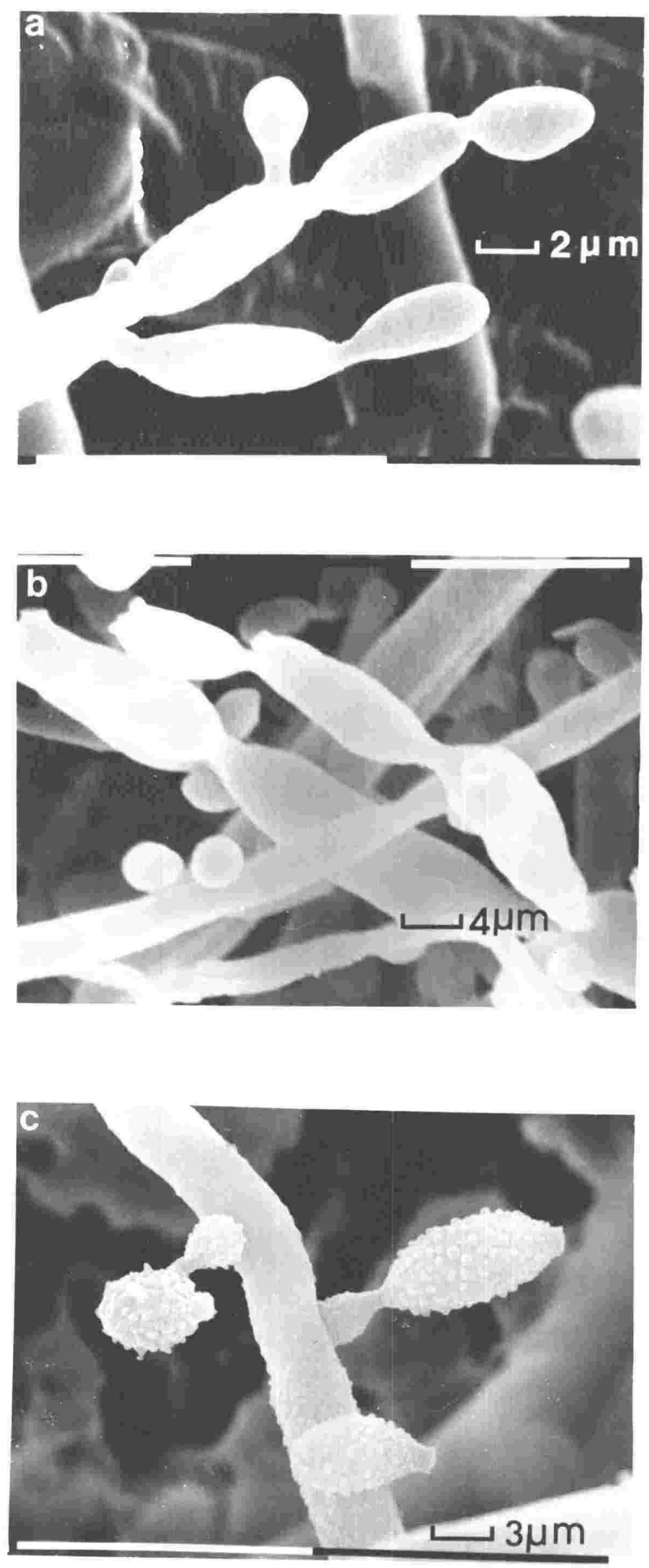

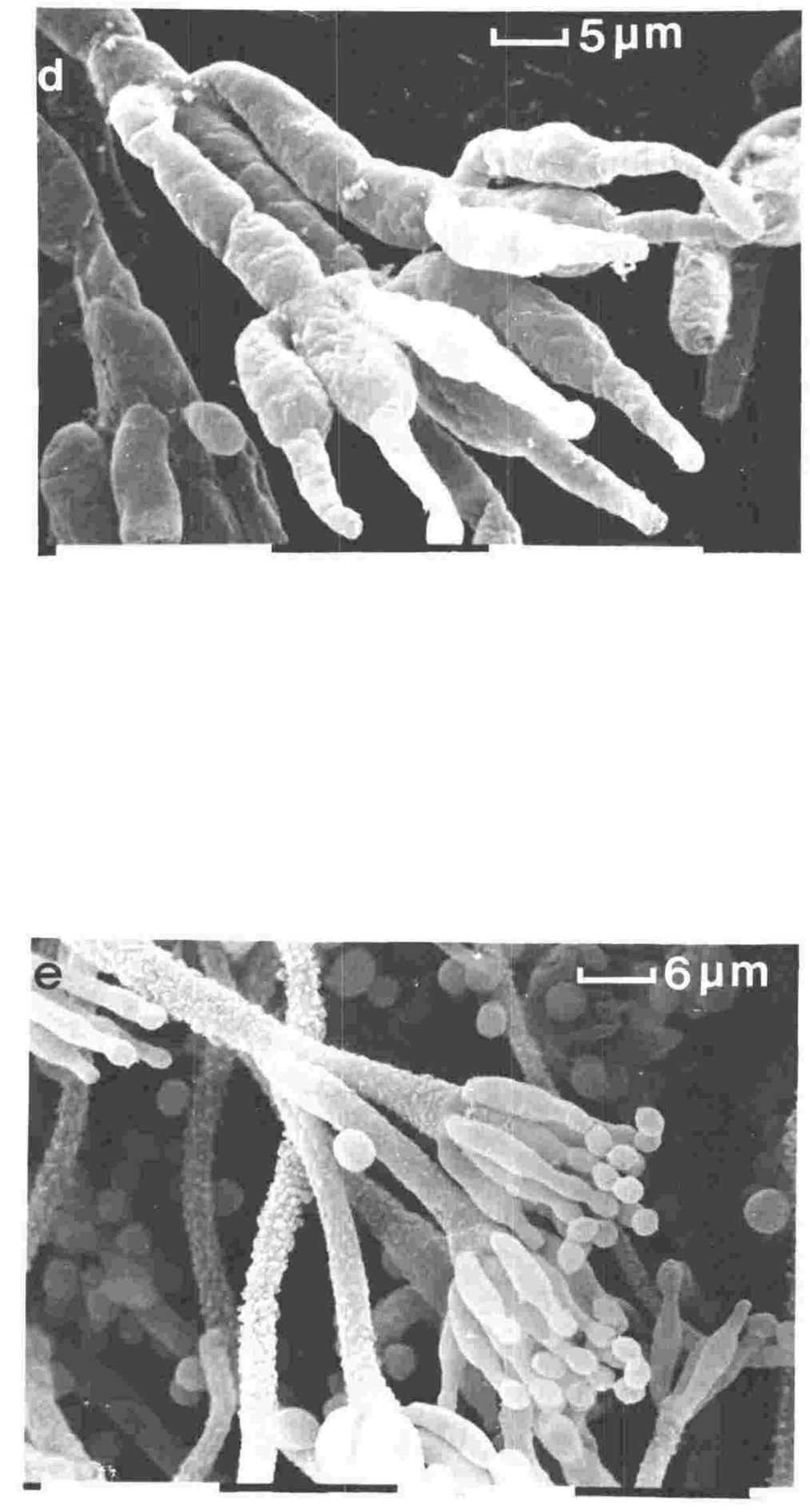
Figure 4.7. Transmission electron micrographs showing the conidia of Cladosporium resinae (ex diesel) prepared using different techniques.

1. Mixtures of glutaraldehyde and osmium tetroxide fixative resulted in cell wall disintegration and the intracellular components appeared dark and granular.

2. Sections fixed in collidine buffer, cell wall was preserved but not the cellular details.

3. Glutaraldehyde and formaldehyde fixative provided more details of the cell wall and intracellular structure. 

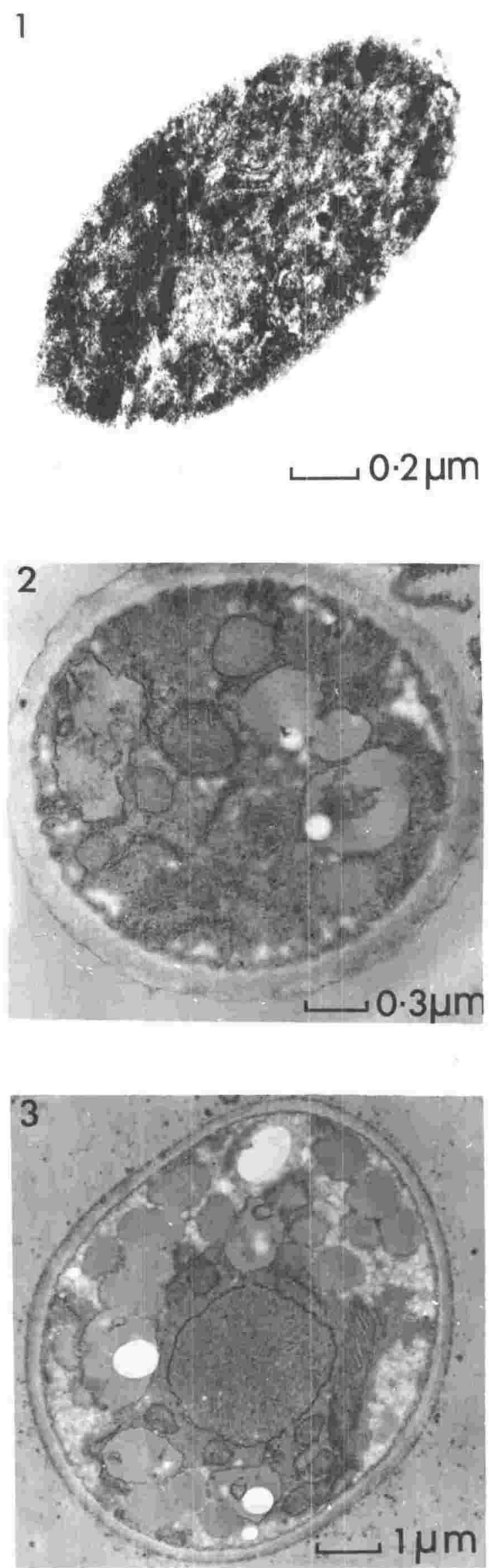
In the sections fixed in collidine buffer, the cell wall was preserved but not the cellular details (figure 4.7-2), but the glutaraldehyde and formaldehyde fixative provided more details of the cell wall and intracellular structure (figure 4.7-3).

Ultrastructure is compared using cultures grown on V-8 juice agar rather than fungal fragments recovered from diesel. A general feature of low power transmission electron micrographs of three Cladosporium spp isolates are shown in figure $4.8 \mathrm{a}, \mathrm{b}$ and $\mathrm{c}$. Conidia have a relatively thick wall within which a substructure can be seen (figure 4.9a, b and c). The cell wall (CW) consists of three distinct layers, an outer electron-transparent layer (A) which can vary in thickness from $600 \mathrm{~nm}-750 \mathrm{~nm}$, a central electron dense layer (B) $(750 \mathrm{~nm}-900 \mathrm{~nm})$ and a thick granular inner layer (C) (120 nm $300 \mathrm{~nm}$ ) which in some cases is closely appressed to the plasma membrane from which it is difficult at times to distinguish.

There are clearly variations in the thickness of each layer but there are no consistent differences between the three species. The plasma membrane (PM) shows frequent irregular invaginations and can be seen to possess the trilamellar structure (figure $4.9 b$ and $c$ ). Some of the membrane irregularities extended quite considerably into the cytoplasm, and structures which may be called lomasomes (LO) appeared as double or multiple membrane surrounded vesicles between the PM and the cell wall (figure 4.9c). Such structures were not restricted to any one species. The cytoplasm is dense and contains all of the expected organelles and structures. The nucleus (N), globose to subglobose was bounded by a nuclear envelope (NF) of two membranes that is frequently continuous with endoplasmic reticulum (ER) (figures $4.10 a$ and $b$ ). Pores (NP) in the nuclear envelope were common. No nuclear division was observed in germinating spores. Other structures observed included numerous mitochondria which varied in sectional view from round to oval. Mitochondria (M) vary little in apparent size, are randomly distributed within the conidia of each spp. and appear to possess plate-1ike cristae (figures 4.10a and b). Endoplasmic reticulum (ER) was sparse but nearly always observed (figure 4.10a). 
Figure 4.8. Low power transmission electron micrographs of:a. Cladosporium resinae ex diesel fuel.

b. Cladosporium resinae ex jet fuel.

c. Cladosporium cladosporioides ex soil. 


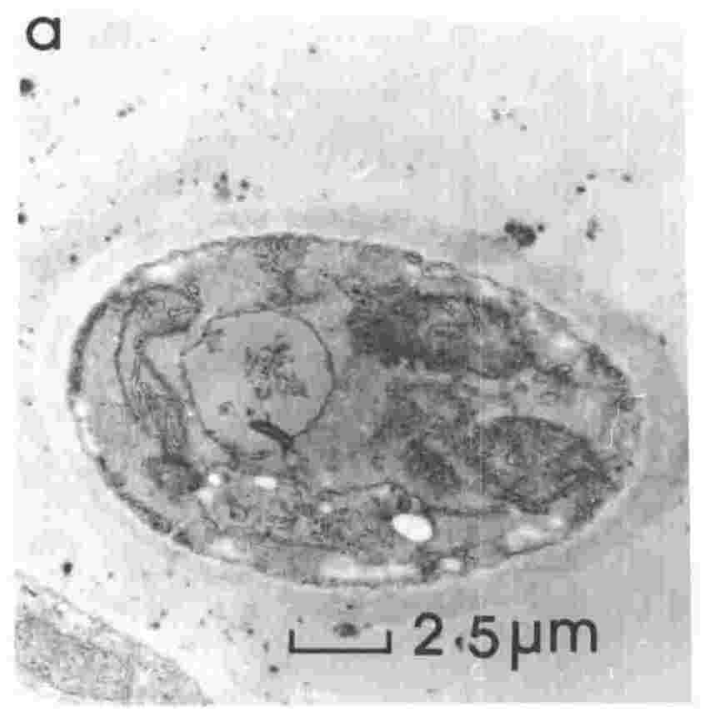

b
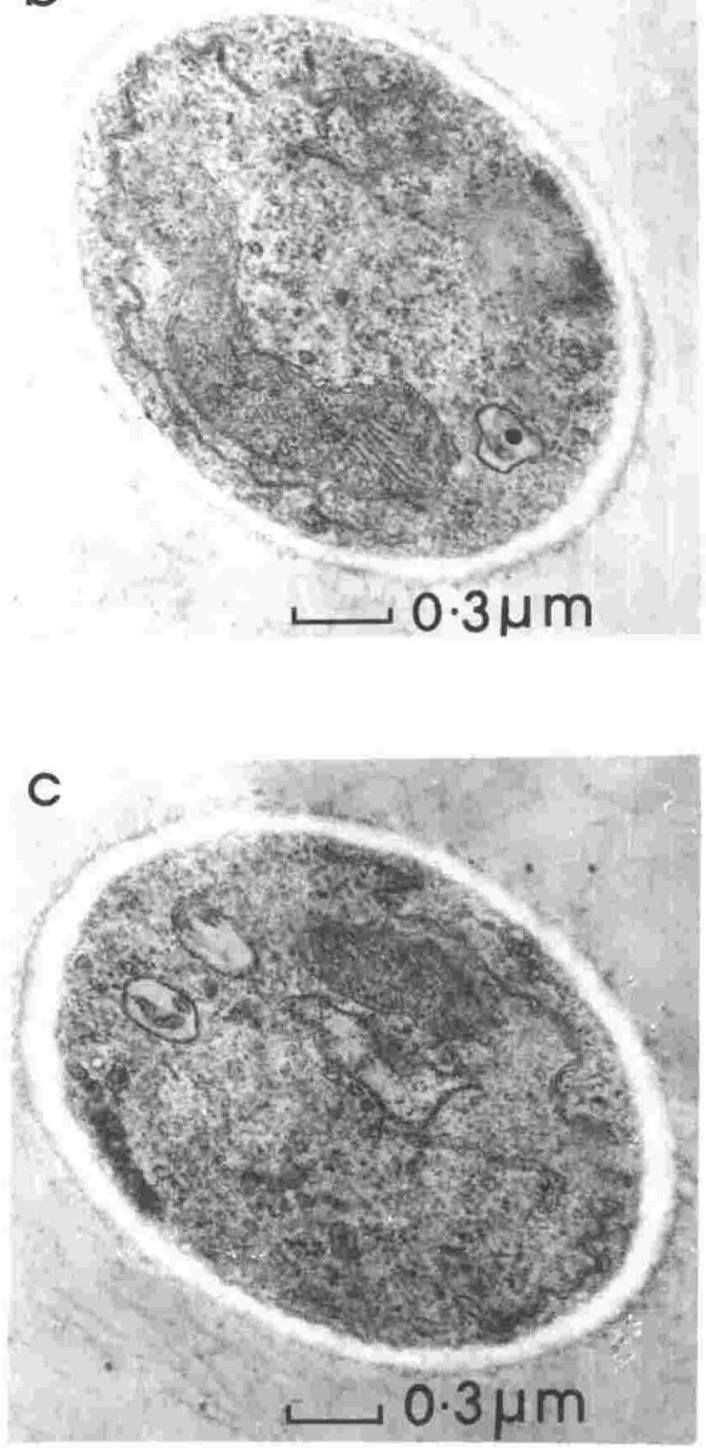
Figure 4.9. a. Transmission electron micrograph showing the cell wall structures of Cladosporium resinae.

b \& c. Electron microgrpahs of plasma membrane of Cladosporium resinae.

$\underline{\text { Key: }}$

$$
\begin{aligned}
& A=\text { outer electron-transparent layer; } \\
& B=\text { central electron-dense layer; } \\
& C=\text { thick granular inner layer; } \\
& \text { PM = plasma membrane; } \\
& C W=\text { cell wall; } \\
& M B=\text { microbody; } \\
& \text { LO = lomasomes. }
\end{aligned}
$$



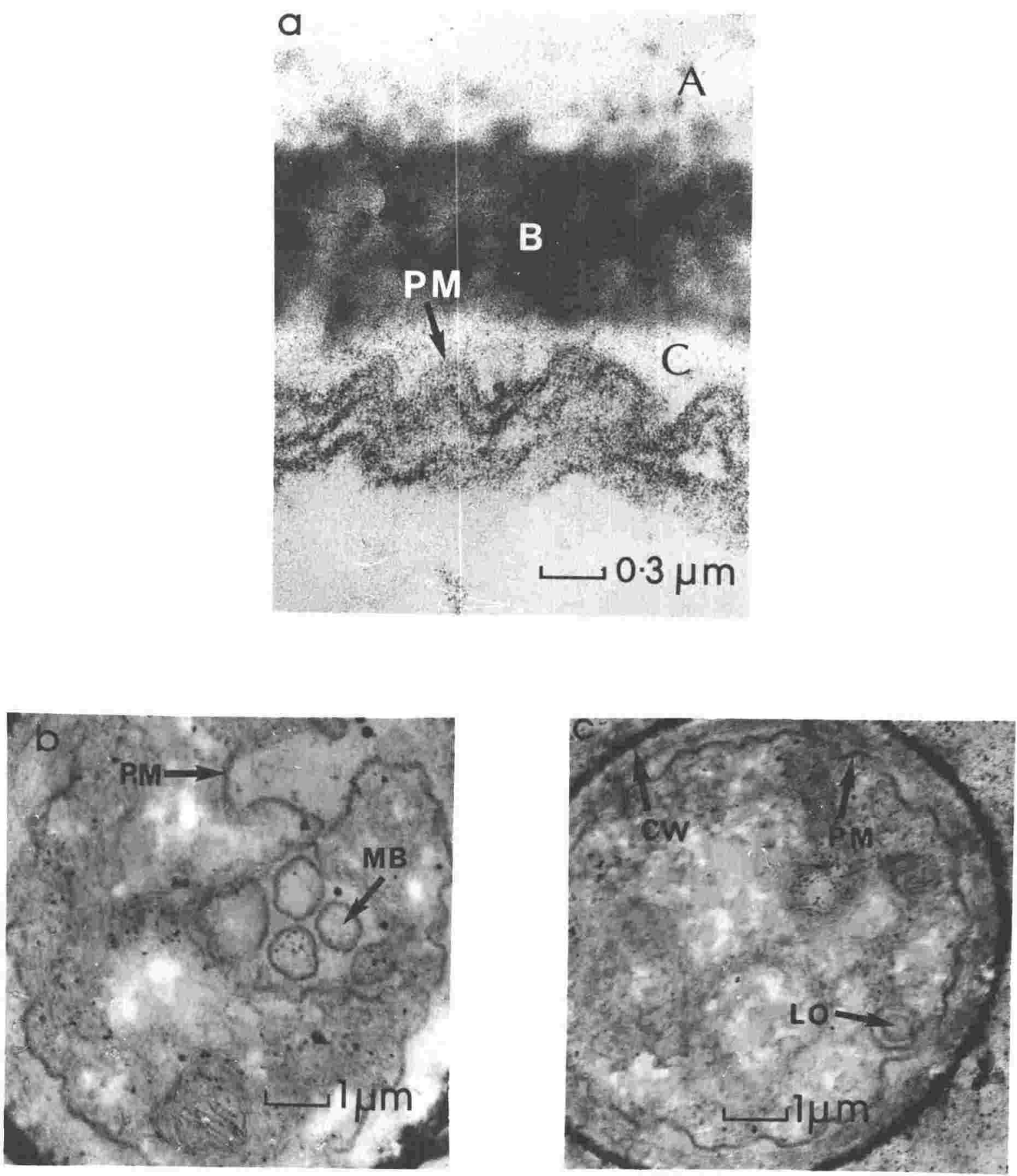
Figure 4.10. a \& b. Electron micrograph of the intracellular conidial structure of Cladosporium resinae.

$\underline{\text { Key: }}$

$$
\begin{aligned}
& \mathrm{ER}=\text { endoplasmic reticulum; } \\
& \mathrm{L}=\text { lipid material; } \\
& \mathrm{M}=\text { mitochondria; } \\
& \mathrm{N}=\text { nucleus; } \\
& \mathrm{NF}=\text { nuclear envelope; } \\
& \mathrm{NP}=\text { nuclear pore; } \\
& \mathrm{T}=\text { tonoplast; } \\
& \mathrm{V}=\text { vacuole. }
\end{aligned}
$$



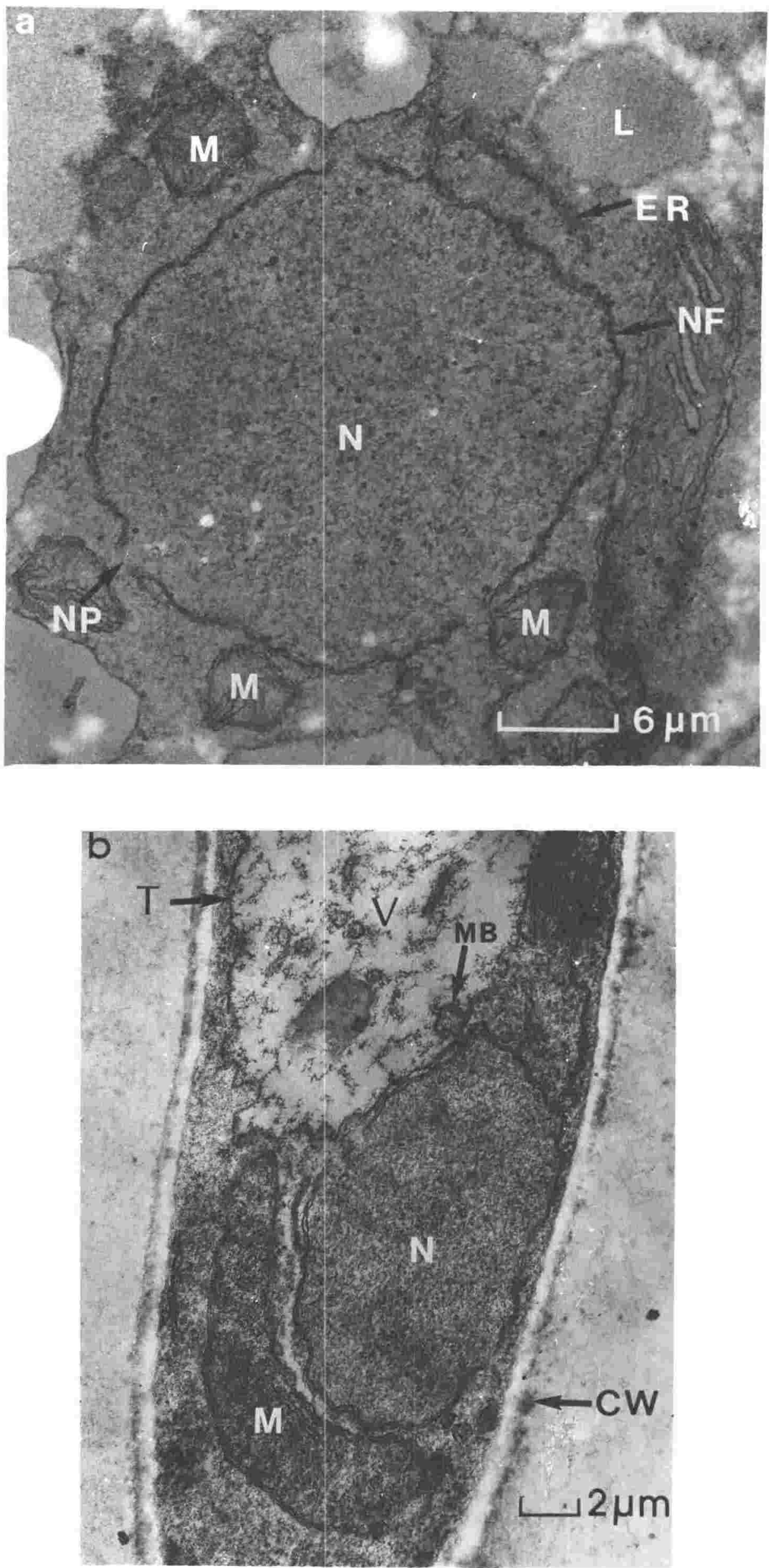
A feature seen in many sections was a highly electron dense cytoplasmic inclusion (LB) which appeared not to be bounded by a membrane and the significance of these bodies is not known (figures 4.1la and b). In a few cases lipid material (L) nearly filled the entire content of the conidia (figure 4.11b). Other similar bodies to lipids may contain proteins and small electron dense particles may be glycogen or other storage granules (arrowed). The conidia of all the Cladosporium spp. showed small intensively stained round bodies in vacuoles and adjacent to septum and these inclusions may be microbodies (MB) (figures $4.12 a$ and $b$ ). In the vegetative hyphae, adjacent to the septum (S), two to three inclusions, which were surrounded by a membrane, appeared similar to woronin bodies (WB) (figure 4.12b). The vacuoles (V) were well-defined by a tonoplast (T) and were particularly evident in germinating spores (figure 4.10b). Sections of the blastospore, ramoconidia and hyphae of all the three species revealed analogous structure. Ramoconidia were typically clavate or cylindrical and contained a greater number of reserve substances (figure $4.11 \mathrm{~b}$ ). 
Figure 4.11. a. Conidia of Cladosporium resinae with the large electron-dense body.

b. Electron micrograph of the ramoconidia of Cladosporium resinae, showing the storage granules (arrowed).

Key:

$L B=$ electron-dense body 

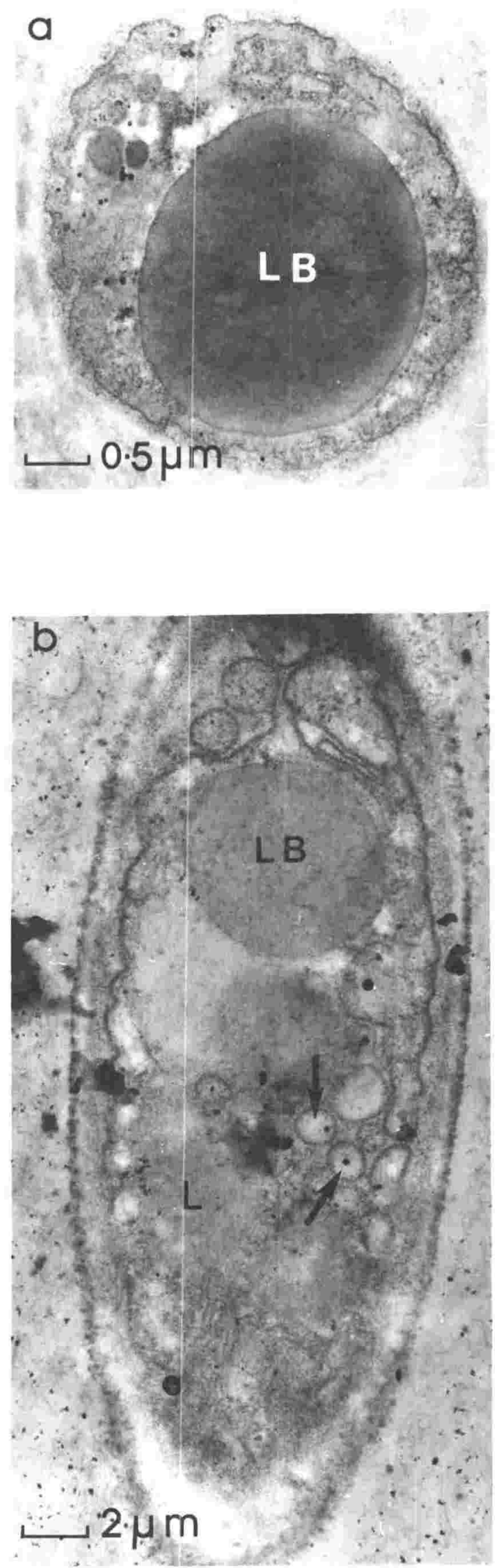
Figure 4.12. a. Microbody adjacent to the septum.

b. Woronin body and electron-dense bodies adjacent to the septum.

$\underline{\text { Key: }}$

$$
\begin{aligned}
& M B=\text { microbody; } \\
& S=\text { septum; } \\
& W B=\text { woronin body } .
\end{aligned}
$$



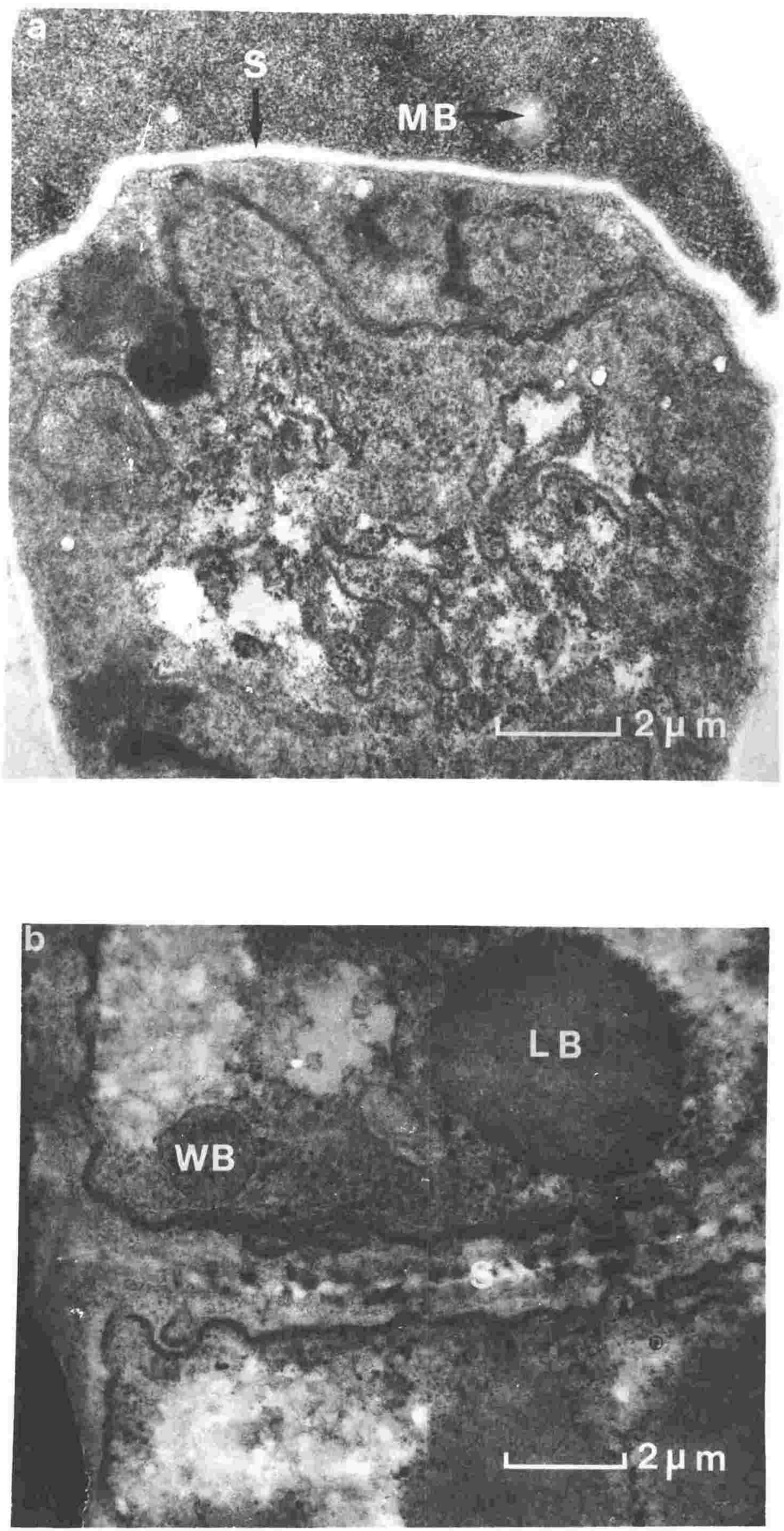


\subsection{Discussion}

The observations made of microbial contamination of the storage tanks were somewhat difficult to interpret due to the uncontrolled nature of many variables such as fungal inoculum, water soluble nutrients, the nature of the fuel hydrocarbon among others. In the laboratory studies of microbial growth, some factors were deliberately controlled to relate to field situations. However there may be some unidentified variables that have not been controlled.

The predominant fungi isolated from diesel fuel in this study were $C_{\text {. resinae, }} P_{\bullet}$ variotii and $P_{\bullet}$ corylophilum with other Penicillium spp. occurring frequently. In all of the laboratory studies the inoculum has been standardized as $10^{6}$ spores per $100 \mathrm{~cm}^{3}$ of hydrocarbon with or without an equal volume of various aqueous phases in glass containers about $50 \%$ full. In all cases these $10^{6}$ spores were added not as an aerial suspension but suspended in $0.1 \mathrm{~cm}^{3}$ of $\mathrm{BH}$ and thus the spores were fully wetted. All fungal growth occurs at the aqueous/fuel interface and this is not surprising since the spores will settle here, as they float in water and water is more dense than hydrocarbon. With the hydrocarbon as the only source of carbon and energy and the aqueous phase as the only source of watersoluble nutrients in the laboratory growth studies, the appearance of extensive mycelium is restricted to this interface. When the aqueous phase contains balanced nutrients as in Bushnell-Haas, growth of all $C$. resinae isolates and of the other fungi is considerable. These growth studies show that apart from $C_{0}$ resinae, both $P_{\bullet}$ variotii and various Penicillium spp. were able to grow in diesel and in the presence of creosote and to utilize these as a source of carbon and energy. Parbery (1970) reported the presence of colonies of $P$. variotii and Penicillium spp. from soil isolates on media containing creosote but no further growth studies were performed. During growth studies in this work a lag phase of two days was

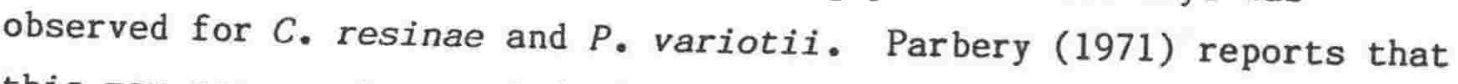
this may represent a period of adaptation and that increasing the inoculum may reduce the lag phase but this suggestion has not been followed experimentally. 
Water enters fuel storage systems in various ways and may be 'pure', contaminated with various soluble materials or be primarily seawater. The results of varying the aqueous phase in these controlled growth studies show clearly how important water-soluble mineral nutrients are and how different fungi require different levels of nutrients. C. resinae appeared to grow only when adequate nutrients were available i.e. in BH/diesel, and was inviable in seawater/diesel after 6 weeks but the prolonged immersion did not affect $P$. corylophilum. $P$. variotii survived in tapwater/diesel, where inadequate mineral nutrient was present. Nicot and Zakartchenko (1966) have noted prolonged (30 months) immersion in a seawater/kerosene phases depressed sporulation and changed conidial morphology. Although with distilled water, where negligible or minimal amount of nutrients were present except for those entrained in the diesel fuel, little if any growth was observed but spores of all the test fungi retained viability for at least 6 weeks.

Since fuel tanks were shown to contain more than one fungal contaminant, the interaction between the predominant or other fungi has been examined with respect to growth and spore viability. In the mixed inoculum studies, growth was observed in the Bushne11Haas/diesel and seawater/diesel. When $C$. resinae and $P_{\text {. corylophilum }}$ were combined, $P$. corylophilum did not survive in $\mathrm{BH} / \mathrm{diesel}$ and conversely $C$. resinae did not grow in seawater/diesel at the same time. This study has revealed that $C$. resinae grew in seawater/diesel after $P$. corylophilum had been grown on the same medium. Again, $C$. resinae was observed to have no metabolic effect on the growth medium especially the BH/diesel medium as the other fungi grew on the same medium when $C$. resinae was eliminated. In some of the growth experiments the final $\mathrm{pH}$ in the aqueous phase dropped marginally. Even a small drop in $\mathrm{pH}$ may play a vital role in the interaction of $C$. resinae with $P$. corylophilum. In other studies, Candida was reported to be present in seawater used in ballasting fuel tanks on ships which produce sufficient acidic metabolic products to lower the $\mathrm{pH}$ to levels where C. resinae could grow (May and Neihof, 1981). Possibly, similar mutual benefits exist between Penicillium corylophilum and $C$. resinae in seawater/diesel fuel. 
The Candida albicans isolate used in this work may not have been necessarily isolated from seawater showed viability in the various aqueous/diesel phases as single inoculum but was reduced or even eliminated by the presence of $C$. resinae, $P$. corylophilum and $P$. variotii. Clearly Candida was unable to interact with the predominant fuel fungi.

C. resinae is widely known as the 'creosote fungus' for it grows on wood impregnated with creosote and utilizes it as food (Christensen et a1., 1942; Sheridan, Tan and Nelson, 1972; Parbery, 1971). Few have studied the effect of hydrocarbon fuels and creosote on Penicillium spp, $P$. variotii and other fungi. It became evident in this study that, like $C$. resinae isolates, $P$. corylophilum and $P$. variotii were able to tolerate creosote up to $1 \%$, including $C$. resinae $f$. resinae ex jet fuel which had not been tested before. Most other Penicillium spp ex diesel were also creosote tolerant and suggest their potential to grow in diesel, provided the favourable conditions exist. Sheridan and Soteros (1974) did not recover any creosote tolerant Alternaria spp. from soil and air in N.Z., but in this study, Alternaria spp. were isolated from air and diesel and both isolates grew in the presence of creosote in V-8 juice agar up to 0.3\%. The tolerance of Alternaria alternata and Cladosporium cladosporioides to creosote has not been recorded before.

According to Sheridan (per. comm.) delayed sampling of jet fuel affected the viability of $C$. resinae spores. This adverse effect was thought to be due to shaking of the fuel samples during transportation. In these studies $C$. resinae spores were viable after shaking and transportation regardless of the type of hydrocarbon fuel. Delayed testing and long term agitation did not affect the recovery of the spores from diesel and jet fuel.

The SEM has confirmed the picture seen in the light microscope with regard to surface structures of conidiophores and conidia of the fungi, except for $P$. corylophilum where the stipe appeared warted and not smooth (Pitt, 1979). 
Sheridan and Troughton (1973) observed warted conidiophores and ramoconidia in $C$. resinae $F$. avellaneum (ex jet fuel), but in this study no such surface ornamentation was obvious. No new fine structures were observed in the SEM. Fungal spores with ornate microscopic surface features can be fully documented by the use of SEM compared with light microscopy.

In the ultrastructural studies, cultures grown on V-8 juice agar provided the source of spores which were used in the laboratory growth studies and these simple observations were to determine possible fine structural features which might explain the ability of spores to survive or to compete in diesel. The buffer solutions used in the study were important to prevent damage and preserve the fine structures of the specimens. The effect of specific ions of the buffer on the fine structures of Cladosporium spp. are not known. Presumably ions in the buffer interact with certain chemical groupings within the specimen which appeared to affect the quality of fixation. Studies with different buffer systems have demonstrated that variations in the specific constitution of the buffer produce significant variations in the staining and appearance of cells and organelles (Wood and Luft, 1965). The efficiency of the buffer system in the present study varied at the different $\mathrm{pH}$ levels. It is now recognized that the osmolarity of a fixative has a direct effect on the appearance of the fixed specimen (Hayat, 1970). The various structures would also differ in the degree of their response to the difference in ion balance between the fixing solution and the organisms' normal environment.

The fixation techniques employed for Cladosporium spp. gave variable results. However, a double fixation by glutaraldehyde and formaldehyde appeared to be more effective in maintaining the constitutents. Mixtures of glutaraldehyde and Osmium tetroxide tend to distort the cell wall and the intracellular structures were dark and granular. But this fixation was ideal for SEM. At present, the knowledge of chemical reaction between the fixing agents and specific cellular constitutents is rather meagre in fungi and interpretation of electron micrographs is dependent upon a better understanding of the chemistry of fixation. 
Studies on the fine structures of Cladosporium spp. showed a thick cell wall composed of several successive layers. Underlying the cell wall, the dark convoluted 'zigzag' membrane (Tan, 1972; Soteros, 1973) was resolved to be a trilamellar plasma membrane. The 'zigzag' membrane may be common to most fungi (Bracker, 1967). The cytoplasmic granular matrix contains organelles characteristic of other fungi.

The nuclear condition of all spores and hyphae of Cladosporium spp. was consistent containing a single nucleus. The pores on the nuclear envelope appear to be potential avenues for exchange of substances between nuclei and cytoplasm (Fawcett, 1966).

The membranous systems of the lomasomes are characteristic structures of fungal cells (Moore, 1965). They arise from the plasma membrane and are not thought to be associated with cell wall formation (Brushaber and Jenkins, 1971). The membranes of lomasomes and the cytoplasmic invagination displayed a trilamellar structure characteristic of the plasma membrane. Since it appears that lomasomes are structures involved in membrane formation, it is considered that the membranous systems of Cladosporium spp. and the invaginations of the plasma membrane may be unique to fungi and not only to hydrocarbon utilizing fungi as suggested by Sheridan, Tan and Nelson (1972).

Cooney, Siporin and Smucker (1980), Smucker and Cooney (1981) compared cells grown on hydrocarbon to those grown in glucose. They observed small spherical, electron-dense bodies in the vacuoles of hydrocarbon grown cells and not in glucose medium, and suggested they were microbodies, the site of initial oxidation of hydrocarbon. They also pointed out the occurrence of large electron-dense bodies in vacuoles of glucose and kerosene grown cells. In this study, such structures were evident in all the Cladosporium spp., despite spores not being processed directly from hydrocarbon medium but from culture medium. The above workers have not made any intracellular comparison between the hydrocarbon and non-hydrocarbon utilizing fungi. 
According to Brenner and Carroll (1968), woronin bodies may function to block the septal pores and regulate or prevent passage of cellular organelles or materials and may also function to occlude the pores. McKeen (1971) ascertained the chemical composition and fine structure of woronin membrane bodies and showed that they occurred in definite location adjacent to septal pores. Cole and Samson (1979) indicated Woronin bodies develop within microbodies as homogenous, electron-opaque inclusions, then move into invaginations of the latter and are pinched off by constriction and fusion of the encompassing membrane. Wergin (1973) and Heath (1977) have pointed out that microbodies are found in animals and plants (Vigil, 1973; Frederick, Gruber and Newcomb, 1975), and fungi (Avers, 1971) have been credited with enzymatic activity, and woronin bodies may possess similar activity. Thus this study did not recognize any intracellular structural difference among the Cladosporium spp. 


\section{$\underline{\text { PART } 2}$}

\section{CONTROL OF MICROBIOLOGICAL CONTAMINATION IN DIESEL FUEL}

This part is concerned with the use of biocides for controlling microbiological contamination in diesel ('dieso') fuel.

\section{MATERIALS and METHODS}

\subsection{Laboratory studies on fuel biocides}

The effects of biocides on spore germination, fungal growth rate and survival were determined using the following techniques.

\subsubsection{Agar/diesel slide technique}

This is a modification of the method described by Smith and Crook (1980) using suspensions of conidia of Cladosporium resinae, Penicillium corylophilum and Paecilomyces variotii on shallow nutrient agar platforms which were then overlaid with diesel (figure 5.1). The agar platforms were placed on the slide in the following manner. Two sterile coverslips $18 \times 18 \mathrm{~mm}$ and each $100 \mu \mathrm{m}$ thick were fixed one above the other in the centre of a normal microscope slide so that it had a raised area of $200 \mu \mathrm{m}$ high. A small volume $\left(0.75 \mathrm{~cm}^{3}\right)$ of molten Bushnell-Haas medium solidified with $2 \%$ agar at $50^{\circ} \mathrm{C}$ was then placed on another culture slide between two stainless steel coupons. Stainless steel $500 \mu \mathrm{m}$ thick was cut into $20 \times 10 \mathrm{~mm}$ coupons and the two coupons were fixed $30 \mathrm{~mm}$ apart across the slide using petroleum jelly. The slide bearing the two coverslips was laid firmly across the two sterile steel coupons with the two coverslips on the underside. When the agar had set the top slide was carefully removed leaving a layer of agar $500 \mu \mathrm{m}$ deep containing a depression, $18 \mathrm{~mm}$ square and $300 \mu \mathrm{m}$ deep, between the steel coupons. The agar surrounding the square depression was then cut away aspetically leaving a platform of $18 \mathrm{~mm}$ square and $300 \mu \mathrm{m}$ deep; the surface was smooth and permitted fungal conidia and hyphae to be observed with ease. 
Figure 5.1. Apparatus for agar slide culture technique (after Smith and Crook, 1980).

Figure 5.2. Sectional view of the small steel tank showing the sampling points.

Key:

$$
\begin{aligned}
& A=\text { top; } \\
& B=\text { middle; } \\
& C=\text { bottom } \\
& D=\text { very bottom }
\end{aligned}
$$



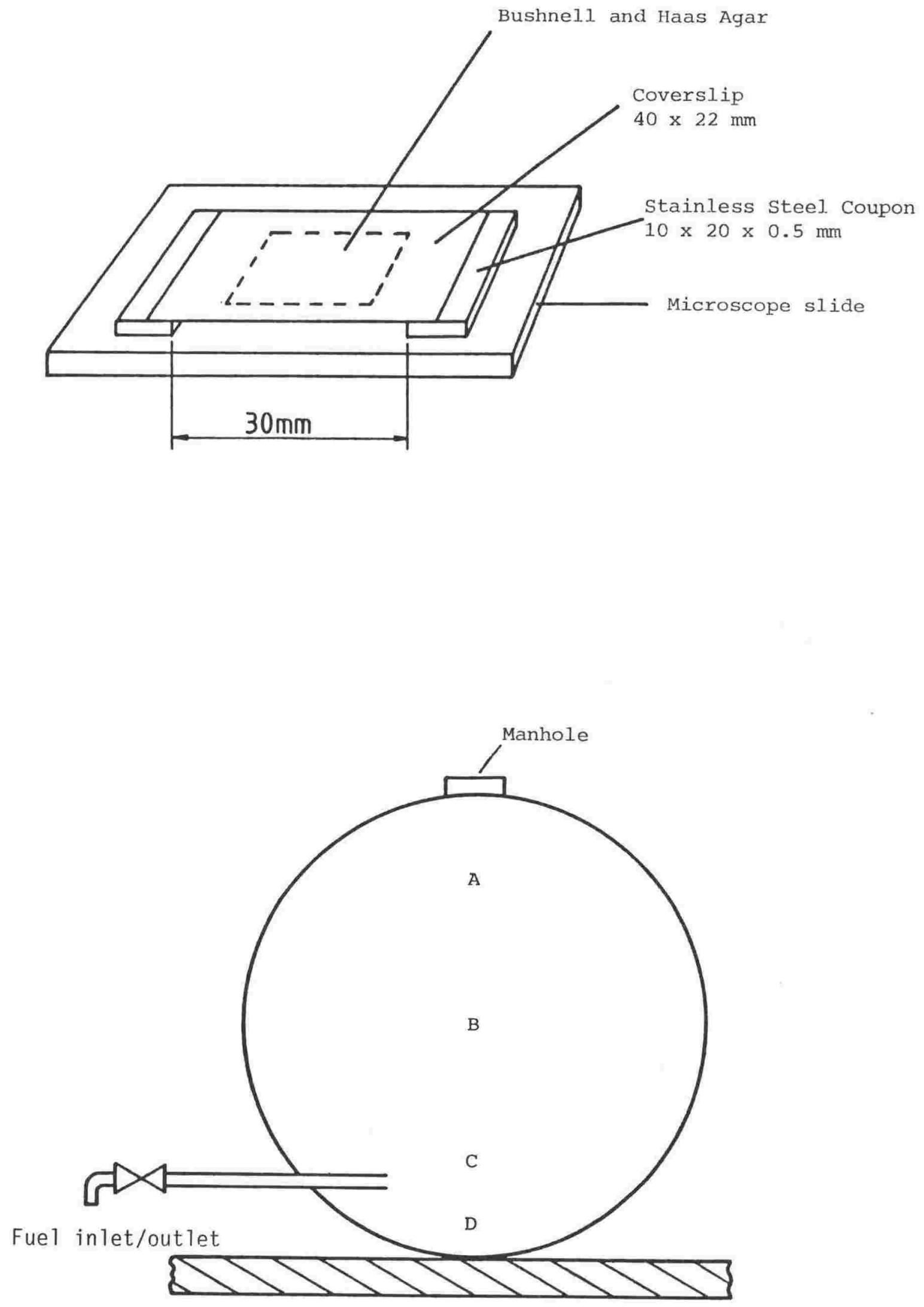
This agar platform was then inoculated with $0.01 \mathrm{~cm}^{3}$ drop (containing $10^{3}$ spores $/ \mathrm{cm}^{3}$ ) of the appropriate conidial suspension (see chapter 2 for details)/was spread on the agar surface. The slide was then left $/$ which for 10 minutes to allow the agar to absorb the free water. A sterile $40 \times 22 \mathrm{~mm}$ No. 1 coverslip was then placed across the steel coupons and about $0.33 \mathrm{~cm}^{3}$ of filter-sterilized diesel fuel containing the various biocides was allowed to run under the coverslip using a sterile Pasteur pipette. The agar cultures (duplicate slides of each fungus per biocide concentration) were placed in covered sterile Petri-dishes and incubated at $25^{\circ} \mathrm{C}$. Germination was determined microscopically daily up to 7 days, being scored on a total of 1000 spores for each treatment and recorded as positive when a developing germ tube had emerged for a distance equal to the width of the spores.

The biocides benomyl, Biobor JF, DML-7, imazalil, Kathon 886 and Proxel AS were used at a final concentration in diesel of 0,5 , 10,50 and $100 \mathrm{ppm}$ and DEGME at concentrations of 0,500, 1000, 2000, 3000 , 4000, $5000 \mathrm{ppm}$. Imazalil and benomyl were solids and all the other products were liquids. A $500 \mathrm{ppm}$ (W/V) stock solution of each in filter-sterilized diesel fuel was prepared and diluted as required. Imazalil was heated to $50^{\circ} \mathrm{C}$ in a water bath before adding the diesel when making up the stock solution. Benomyl required a different procedure due to its low solubility in both water and diesel. It is soluble in both dimethyl formamide (DMF) and dimethyl sulphoxide (DMSO) (Smith, per. comm.) but the latter was not used because it was thought that the sulphur content might stimulate sulphur bacteria in the tanks. The stock solution of benomyl was prepared by dissolving $25 \mathrm{mg}$ in $0.5 \mathrm{~cm}^{3}$ of DMF, mixing with $5 \mathrm{~cm}^{3}$ of diesel and making up to a volume of $50 \mathrm{~cm}^{3}$ with diesel to give the $500 \mathrm{ppm}$ solution required.

\subsubsection{Poison plate test (Growth rate)}

The effect of biocides, incorporated into nutrient agar on the growth of colonies of $C$. resinae, $P$. variotii, Penicillium

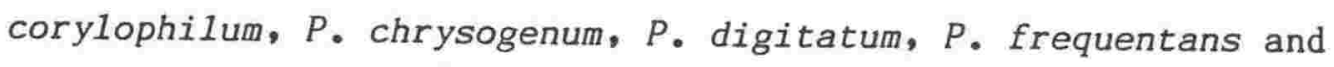
$P$. spinulosum was determined over a 7 day period. 
The additives were incorporated immediately into the sterile molten malt extract agar $(2 \%)$ at $50^{\circ} \mathrm{C}$ at concentrations of $0,10,50,100$, 200 and $300 \mathrm{ppm}$ except for DEGME and EGME. These were used at the higher concentrations of $1000,2000,3000,4000,5000 \mathrm{ppm}(0.1$ $0.5 \%)$. The mixtures were gently shaken to distribute the biocides and plates poured. $5 \mathrm{~mm}$ plugs from the periphery of 2 week old actively growing cultures were placed upside down centrally in each Petri-dish. Colony diameter from five replicate dishes was measured daily for each treatment.

The effect of biocides on the following seven isolates of $C$. resinae was studied: $C$. resinae $f$, avellaneum ex soil (C1); R5 ex feather; ex Air at Devonport; ex untreated diesel (isolated in this study); ex jet fuel (K1OF); ex Diesel (D1); ex Australian army culture. Isolates of $C$. resinae and $P$. variotii from the two diesel tanks obtained during and after treatment for up to 5 months with Biobor JF and DEGME were also studied.

The effect of mixtures of benomy 1 (B) and imazalil (I) on the

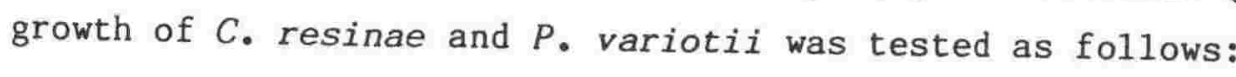

- total concentration 5 ppm with B decreasing from 5 to 0 while I increased from 0 to 5 in steps of $1 \mathrm{ppm}$.

- total concentration $10 \mathrm{ppm}$ with B decreasing from 10 to 0 , while I increased from 0 to 10 in steps of $1 \mathrm{ppm}$.

- total concentration $50 \mathrm{ppm}$ with I at $5,10,20,25,30,40,45$ and 50 and $B$ decreasing in proportion.

- total concentration 100 ppm with B decreasing from 100 to 0 ppm while I increased from 0 to $100 \mathrm{ppm}$ in steps of $10 \mathrm{ppm}$.

- total concentration 200 ppm with B decreasing from 200 to 0 while I increased from 0 to 200 in steps of $10 \mathrm{ppm}$.

Growth was assessed by measuring the colony diameter after 8 days. 


\subsubsection{The effects of biocides on fungal growth and survival in}

$\underline{\mathrm{BH} / \text { diese } 1 \text { phases }}$

The activities of the biocides listed in 5.1.1 were tested as follows:

(i) To determine the growth and survival of mixed spore inoculum. Mixed spore inoculum of $C$. resinae, $P_{\bullet}$. variotii, Penicillium corylophilum, $P_{\text {. chrysogenum, }} P_{\text {. spinulosum and }} P_{\text {. digitatum }}$ was inoculated in $\left(100 \mathrm{~cm}^{3}\right.$ ) media consisting of autoclaved Bushnell-Haas mineral medium and filter-sterilized diesel fuel in the following proportions $5: 0,5: 1,5: 2,5: 4,5: 5,4: 5,3: 5$, $2: 5,1: 5$ and $0: 5$. The tests were set up in $200 \mathrm{~cm}^{3}$ medical flat bottles (sterilized in an oven at $200^{\circ} \mathrm{C}$ for 3 hours). Benomy 1 (dissolved in DMF), DML-7, imazalil and Proxel AS were dissolved in diesel and added to give final concentrations of $0,50,200,300 \mathrm{ppm}$. Biobor JF and DEGME concentrations were $0,125,170$ and $270 \mathrm{ppm}$ (commercially recommended) and 0, 1000, 2000 and $3000 \mathrm{ppm}$ respectively. After shaking, $0.1 \mathrm{~cm}^{3}$ of fungal spore suspension (containing approximately $10^{5}$ spores $/ \mathrm{cm}^{3}$ ) in $\mathrm{BH}$ was inoculated into each medical flat. The bottles were shaken again and the aluminium caps with the rubber liner removed, were screwed on loosely and the bottles incubated at $25^{\circ} \mathrm{C}$ for 6 weeks.

Where growth had occurred, the mycelial mat produced by the fungi was harvested by filtering using sterile No. 1 Whatman filter papers ( $50 \mathrm{~mm}$ diameter). Where there was no mycelial mat formation, cultures were shaken, $5 \mathrm{~cm}^{3}$ of the BH/diesel sample was placed in duplicate sterile Petri-dishes and V-8 juice agar poured over the sample and shaken gently. The plates were incubated at $25^{\circ} \mathrm{C}$ and scored for the number of fungal colonies produced. 
To observe the effect of EGME and DEGME on C. resinae at reduced temperatures

The sterile $\mathrm{BH} / \mathrm{diesel}$ fuel was used at $1: 1$ ratio in sterile 200 $\mathrm{cm}^{3}$ medical flat bottles as described in (i). The BH/fuel was inoculated with $0.1 \mathrm{~cm}^{3} C$. resinae spore suspension in $\mathrm{BH}$ (approximately $17 \times 10^{5}$ spores $/ \mathrm{cm}^{3}$ ). EGME and DEGME were added to give concentrations of $0,300,500,100010,000$ ppm $(0.03-$ $1 \%$ ). The bottles were incubated at $4^{\circ} \mathrm{C}$ for 4 weeks and then transferred to laboratory temperature $\left(18-19^{\circ} \mathrm{C}\right)$ and kept for a further 4 weeks to observe whether vigorous growth had occurred by the end of the 8 weeks. The mycelial mat produced by the fungus was harvested and assessed as above.

(iii) To determine whether batches of DEGME varied in biocidal activity

An Australian diesel fuel isolate, a N.Z. diesel fuel isolate and a N.Z. jet fuel isolate of $C$. resinae were found to be insensitive to the DEGME sample used in the laboratory. The new batch of DEGME purchased for the field trial was tested using this culture method against these $C$. resinae isolates to investigate whether the response of the test organism varied with different samples of DEGME.

\subsubsection{Large volume liquid fuel : biocide tests}

Fuel (2 litres) obtained from the main storage tank 2 was placed in sterile 'Winchester quart' bottles (capacity 2.25 litres). Before the addition of the biocides, three $50 \mathrm{ml}$ samples of the fuel were filtered to discover the level of microbial contamination. The biocides dissolved in sterile diesel were added to the fuel to give a final concentration at $0,50,100,200,300 \mathrm{ppm}$ for DML-7, Proxe1 AS, imazalil, benomy1, Biobor JF, Kathon 886 but for EGME and DEGME at 0 , $500,1000,2000,3000 \mathrm{ppm}(0.05-0.3 \%)$. 24 hours after the incorporation of the biocide, three $50 \mathrm{~cm}^{3}$ samples of fuel were filtered. This was repeated daily for seven days using $0.45 \mu \mathrm{m}$ membrane filters. The membrane filters were placed in sterile Petri-dishes and V-8 juice agar poured over the filters. The plates were incubated at $25^{\circ} \mathrm{C}$ for 5 days before colonies were counted. 


\subsubsection{Intermittent exposure of the three common fuel fungi to biocides in diesel fuel}

The method was described by Elphick and Hunter (1968) for jet fuel and modifications were made whenever necessary. $30 \mathrm{~mm}$ circles were cut from no. 1 Whatman filter paper. The filter paper circles were autoclaved for 15 minutes at $103 \mathrm{kPa}$ (15 p.s.i.). To these sterile circles were applied $0.3 \mathrm{~cm}^{3}$ of a mixed spore suspension (approximately $10^{7}$ spores $/ \mathrm{cm}^{3}$ ) of $C$. resinae, $P_{\text {. variotii and }}$ $P$. corylophilum in sterile water, a volume sufficient just to saturate the circles. The circles were placed in sterile $140 \mathrm{~mm}$ Petri-dishes, arranged concentrically without overlapping, and a $5 \mathrm{~mm}$ deep layer of filter-sterilized fuel carefully poured over them. The dishes were incubated at $25^{\circ} \mathrm{C}$. After 7 days, a further $0.3 \mathrm{~cm}^{3}$ of sterile water was added to each filter paper. After a further 14 days incubation, vigorous mixed colonies of the fungi were growing at the centre of each circle.

The mixed colonies were then immersed in fuel containing the biocides for 24 hours, held in $200 \mathrm{~cm}^{3}$ beakers and covered with sterile aluminium foil. The products used were Biobor JF, benomy1, DML-7, imazalil, Kathon 886, Proxel AS at concentrations of 0, 50, $100,200,300 \mathrm{ppm}$. Filter paper circles were removed after 24 hours and, without washing, placed in untreated sterile fuel for 6 days. They were then removed and immersed once more in biocide-treated fuel for a second 24 hour period, the process being repeated to give a maximum of six immersion periods in biocide-treated fuel, a total of 144 hours over a 6 week period. Three filter paper circles carrying colonies were removed after each 6 day period in untreated fuel, washed with sterile water and placed in sterile Petri-dishes. Malt extract agar was poured over the filters. They were assessed for relative growth after 5 days incubation at $25^{\circ} \mathrm{C}$. 


\subsection{Field evaluation of the fuel biocides}

\subsubsection{Biocide effects on microbial contamination}

(i) Tank description

Uncoated steel tanks at Devonport Naval Base, Auckland, were used for the large scale biocide testing. Each of the cylindrical tanks was approximately $4.57 \mathrm{~m}$ long and $2.74 \mathrm{~m}$ wide, with a capacity of 25,500 litres of diesel fuel. They faced south and were thus completely sheltered by the cliffs behind from direct sun. Usually three tanks were used, two treated and the third as an untreated control. The tanks were drained, biocides added and then refilled with diesel which completely mixed the biocides with the fuel.

(ii) Biocides

DEGME and Biobor JF were added to give final tank concentrations of $3000 \mathrm{ppm}(0.3 \%)$ and $270 \mathrm{ppm}(0.027 \%)$ respectively, DML-7 and Proxel AS at $300 \mathrm{ppm}$; benomy1 at $50 \mathrm{ppm}$ and imazalil at $200 \mathrm{ppm}$. Benomyl was dissolved in DMF, a11 others were added at the required final concentration directly to the tanks. Benomyl was used at a low concentration because it was found to be controlling the predominant fuel contaminants between $10 \mathrm{ppm}$ and $50 \mathrm{ppm}$ in the laboratory.

\section{(iii) Sampling}

Samples were collected and transferred into sterile preserving jars as described in Part 1. Samples were collected before (the untreated fuel) and about $21 / 2$ hours after addition of biocides (allowing time for the product to settle) and tested immediately in Auckland and after 24 hours in Wellington for the viable fungal contaminants in the DEGME and Biobor JF treated samples. The treated samples were tested immediately and after 24 hours, to observe whether there was any change in the fungal contamination level. Later it was found, that delayed sampling did not have any effect on the microbiological contaminants. 
In the case of DML-7, Proxel AS, benomyl and imazalil, the untreated fuel samples were collected and tested immediately in Auckland. The treated tanks were allowed to settle for 24 hours before sampling, and samples were tested in Wellington. The source of fuel was from the main storage tank 2 and it was sampled and tested before pumping into the tanks. The subsequent samples from the treated and untreated tanks were collected fortnightly from the top (A), middle (B), bottom (C) and infrequently from the very bottom (D) as shown in figure 5.2. The samples were tested in Wellington for a period of from 16 to 20 weeks after the addition of biocides. The tanks have the sullage bottom (D) (undrainable bottom) which were only drained manually and extended $305 \mathrm{~mm}$ high from the very bottom to the point at which fuel is normally withdrawn (C).

Initially only a single sample $\left(1000 \mathrm{~cm}^{3}\right)$ was collected from the above levels. Later, it was noticed that the number of colony forming units fluctuated greatly between levels in the tanks at the same sampling time, so triplicate samples $\left(1000 \mathrm{~cm}^{3}\right)$ were collected separately at each level.

\subsubsection{Microbial detection}

The fuel was thoroughly hand shaken before subjecting to the membrane filtration technique (refer Part 1). The size of the 10 replicate fuel samples filtered was $100 \mathrm{~cm}^{3}$.

Since the presence of biocide on the filters or in fungal fragments may affect the detection of viable fungi, the following tests were performed on filters before covering with the V- 8 juice agar, each being replicated 15 times.

(a) Filters plated without washing.

(b) Filters were washed with sterile distilled water before plating. 
(c) The filters were washed with a detergent triton $X-100$ (20 $\mathrm{cm}^{3}$ sterile distilled water mixed with $0.1 \mathrm{~cm}^{3}$ triton $X-$ 100), mainly to remove any residue of DEGME and Biobor JF.

(d) V-8 juice agar containing $0.1 \%$ creosote was poured over the unwashed filters.

The plates were incubated at $25^{\circ} \mathrm{C}$ and observed after 5 days for fungal colonies. There were only minor differences between treatments, with treatment ' $C$ ' appearing most satisfactory. This detergent wash was used for all subsequent samples. Whenever free water was observed in the very bottom samples, the $\mathrm{pH}$ was read using the $\mathrm{pH}$ meter.

\subsubsection{Effects of biocides}

\subsubsection{Engine performance and combustion chamber components}

The object of engine running tests with dosed fuel was to ascertain whether the chemical products under test had any effect on the performance of diesel engines and engine components. The engine trials were run at the Defence Scientific Establishment (D.S.E.) at Devonport, using one single cylinder Enfield VSI engine. The motor drives an electrical alternator which is off-loaded onto a bank of resistance heaters. The engine was rated to run at a constant speed of $1400 \mathrm{rpm}$ and it developed 4474 Watts ( $6 \mathrm{hp}$ ). The alternator generated a maximum of 2780 Volt Amps (VA) which could be translated into 2780 Watts if used to power a bank of electrical resistance heaters, because of the unity power-factor under this type of loading. To the test diesel generator was fitted $2 \mathrm{AC}$ meters, one to read 0-250 Volts and the other to read 0-20 Amps. The test engine was placed in a weather-proof bay and an additional metering tank (snap tank) was connected in parallel with the main fuel tank. This permitted the recording of fuel consumption rate while the engine was running at a fixed power output. Any changes to the thermal efficiency of fuel caused by the additive was measured by this method. After each major fuel/biocide trial, the cylinder head (see figure 5.3) was removed so that deposits could be visually examined and any relevant measurements made. 
Figure 5.3. The cylinder head of the Enfield VS1 engine. 


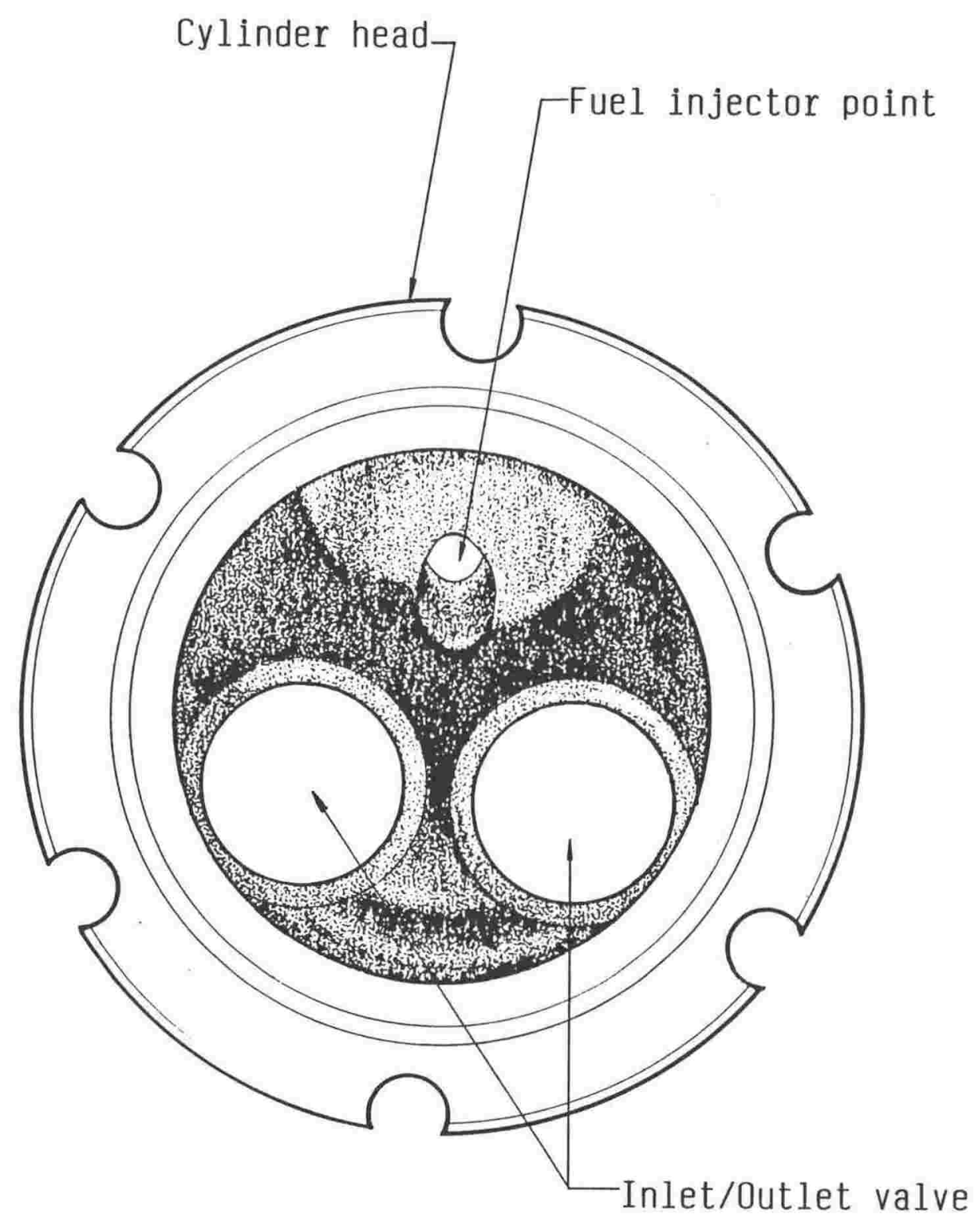


Each biocide/fuel mixture was tested over a two or three day continuous running period and fuel consumption rate and oil pressure measured hourly. All biocides used in the field trial including Kathon 886 were tested in the engine and in the following corrosion test.

\subsubsection{Corrosion test}

A standard method for detection of copper corrosion from petroleum products by the copper strip tarnish test, as described in the IP 154/84, ASTM D130-83 was followed.

\subsubsection{Water and biocide content of fuel samples}

\subsubsection{Determination of water content}

The total water (free and dissolved water) in most of the fuel samples from the three tanks was determined at D.S.E. by the Kar 1 Fisher method (ASTM D17 44-64).

\subsubsection{Determination of Partition Coefficient}

Biobor JF - Boron in Biobor products in diesel was determined by the potentiometric method (Park, 1975) at B.P. N.Z. Ltd.

DEGME - the method used was a standard technique for Icing Inhibitor using a refractometer, DEF (Aust.) 5240.

Proxel AS was analysed by high performance liquid chromatography (HPLC) at Chemistry Division, DSIR as follows:

Ten millilitres of diesel sample and $10 \mathrm{~cm}^{3}$ of distilled water were shaken together vigorously for one minute in a $25 \mathrm{~cm}^{3}$ vial and the two phases were allowed to separate completely. The aqueous phase was then analysed by HPLC on a Hichrom $\mathrm{C} 18$ reverse phase column (50 mm $\times 8 \mathrm{~mm}$ ) using methanol-water-orthophosphoric acid 50:50:0.25 $\mathrm{V} / \mathrm{V} / \mathrm{V}$ at $3 \mathrm{~cm}^{3} / \mathrm{min}$ as mobile phase. $500 \mu 1$ injections were made and Proxel AS was detected by UV absorbance at $240 \mathrm{~nm}$. Concentrations were calculated using peak heights calibrated using standards. Analyses were reproducible to within $\pm 2 \%$, and were carried out in duplicate. 
Experiments using authentic mixtures showed that in a 50:50 mixture of diesel and water, $(80 \pm 1) \%$ of the Proxel was in the aqueous phase and only $20 \%$ in the diesel.

DML-7 - HPLC and capillary gas chromatography (GLC) were used for quantitation of active components from DML-7 in aqueous and hydrocarbon phases at Industrial Processing Division (IPD), DSIR. The major components identified were:

- Aromatic Hydrocarbon Solvent (Boiling Range $160^{\circ} \mathrm{C} \rightarrow 220^{\circ} \mathrm{C}$ )

- Unknown Compound (water soluble, Boiling Point 120 $\mathrm{C}$ )

- Diethylene glycol-mono methyl ether (Boiling Point $194^{\circ} \mathrm{C}$ )

- Methylene bis thiocyanate (melting Point $103^{\circ} \mathrm{C}$ ).

The first three components could be quantified in both hydrocarbon and aqueous phases using GLC, but the methylene bis thiocyanate required aqueous extraction followed by HPLC.

Once the composition of the DML-7 was determined, it was decided that methylene bis thiocyanate was the only component worth quantifying. It was analysed as follows in the $200 \mathrm{~cm}^{3}$ diesel fuel from a 25,500 litre storage tank initially dosed with $300 \mathrm{ppm}$ of DML-7.

The DML-7 sample was analysed by GLC using a non-polar capillary column with flame ionisation detection. Water was added to the DML-7 and both the hydrocarbon phase and the aqueous phase analysed. A sample of the biocide was washed with water and pentane to extract the partially soluble solid component. The melting point of the crystals was determined. A sample of the diesel fuel was analysed by capillary GLC. DML-7 standards were prepared by adding $300 \mathrm{ppm}$ of biocide to diesel, consequently methylene bis thiocyanate results are reported as ppm biocide because the methylene bis thiocyanate content is not accurately known. Some of the extracted crystals were used to estimate the amount of methylene bis thiocyanate in the DML-7 biocide. The result indicated the biocide contained $~ 8 \%$ mass/mass of methylene bis thiocyanate, however this value is not precise due to interference from other biocide components. 
40 grams of the submitted fuel samples were extracted with $3 \times 4 \mathrm{ml}$ of distilled water and the combined extracts analysed by reverse phase HPLC using ultraviolet absorption detection at $254 \mathrm{~nm}$. Two fuel samples contained small quantities of separated aqueous phase and these volumes were measured using a microlitre syringe. The significant concentrations of solid corrosion products in the aqueous phase prevented HPLC analysis.

The extraction of biocide from diesel into water was measured by adding 1 part water to 250 parts of diesel containing $300 \mathrm{ppm}$ DML-7. The mixture was stirred for 4 hours. The extraction was repeated on the same sample.

Imazalil content was not measured specifically in fuel samples but since imazalil is a non-polar substance (evident from the chemical formula) one can predict it to be more soluble in fuel than in water (R.D. Wilson, Chemistry Division, DSIR, per. comm.). A manufacturer representative (Janssen Pharmaceutica, per. comm.) advised that imazalil was more soluble in octanol than in water phases from their determination of partition coefficient. (See appendix 5.1 for octanol-water partition coefficient).

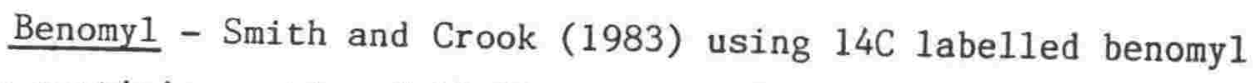
found a partition ratio of 10:90 water to diesel fuel after equilibration. 
In this study eight chemical compounds were tested as biocides in the laboratory and six in the field. Their overall performance is summarised in table 6.1 .

\subsection{SECTION I - Laboratory studies of biocide effects}

\subsubsection{Agar/diesel slide technique}

Table 6.2 shows that germination of conidia of the three major fungal contaminants was evident within 24 hours when placed onto the interface between $\mathrm{BH}$ mineral salt agar and diesel. The germination was complete within 48 hours.

Neither DEGME nor Biobor JF affected germination significantly although the latter gave a slight delay in germination of $C$. resinae at the higher concentrations.

DML-7 at all concentrations and Proxel AS at the higher concentrations delayed germination of $C$. resinae and $P$. corylophilum but not Paecilomyces variotii. By 96 hours germination was $100 \%$ for a11 three fungi.

At or above $100 \mathrm{ppm}$, benomyl inhibited germination completely in all three fungi. C. resinae was most sensitive to benomyl being completely inhibited at $10 \mathrm{ppm}$ with delayed germination at $5 \mathrm{ppm}$.

Imazalil at or above $50 \mathrm{ppm}$ inhibited germination in $C$. resinae and $P$. corylophilum. Paecilomyces variotii was very insensitive with complete germination after 96 hours at all concentrations.

The agar slide technique permitted observation of germination and growth of spores as they produce individual colonies. Hyphal growth in untreated diesel resulted in an interwoven mycelial mat in which no individual colonies could be distinguished within 48 hours. The type of hyphal growth and the formation of conidiophores and conidia could be readily observed using this technique. 


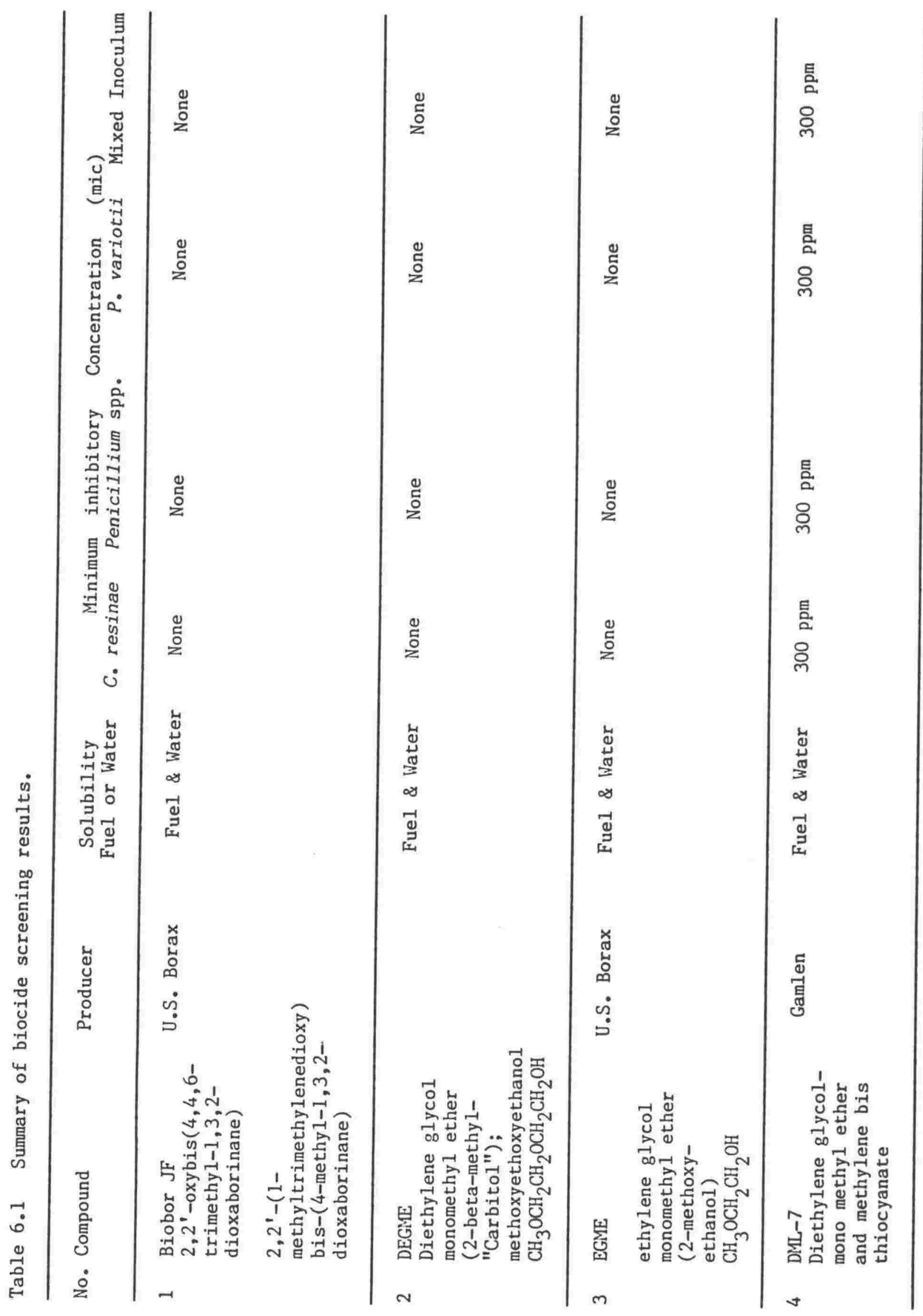




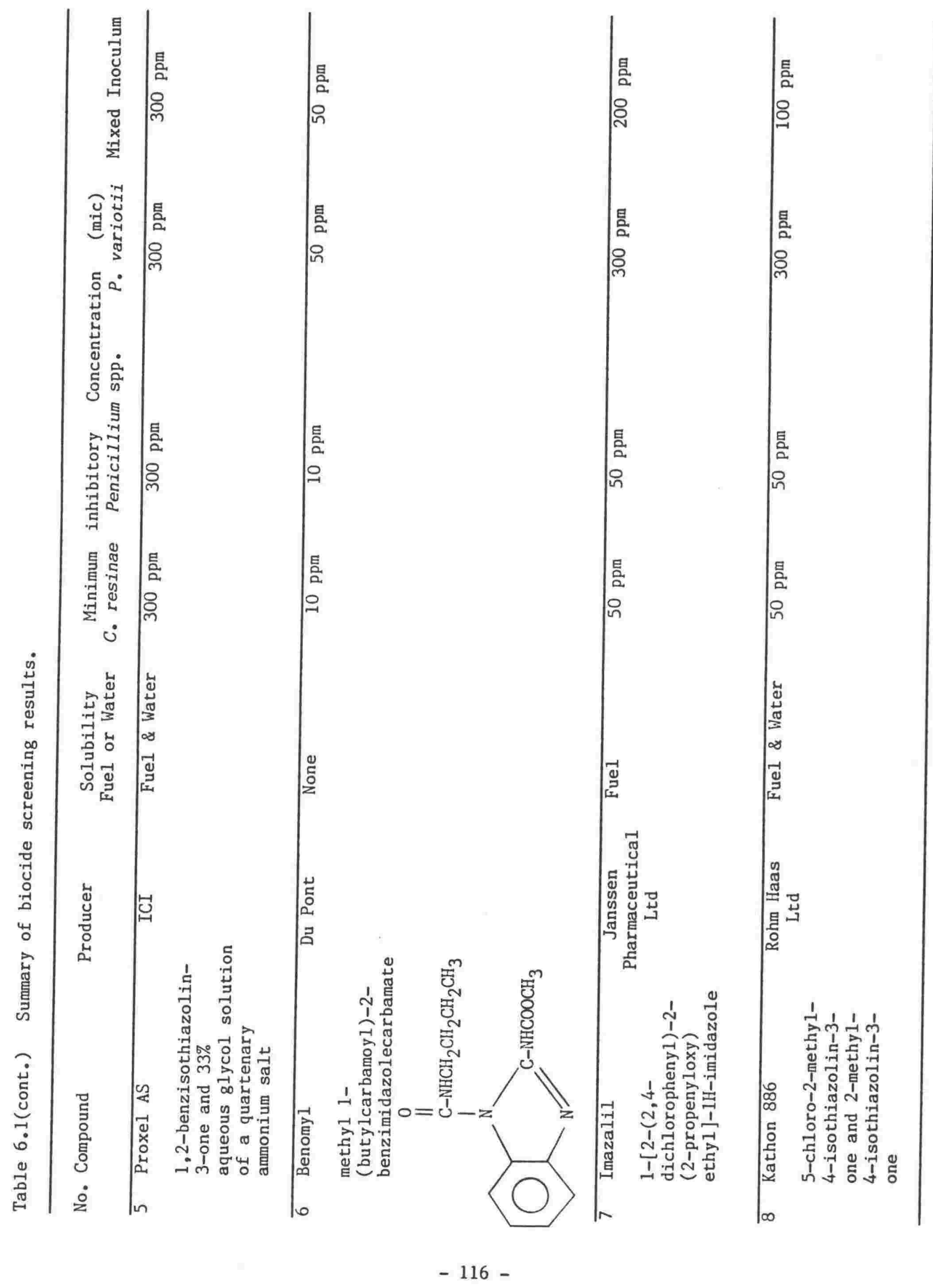




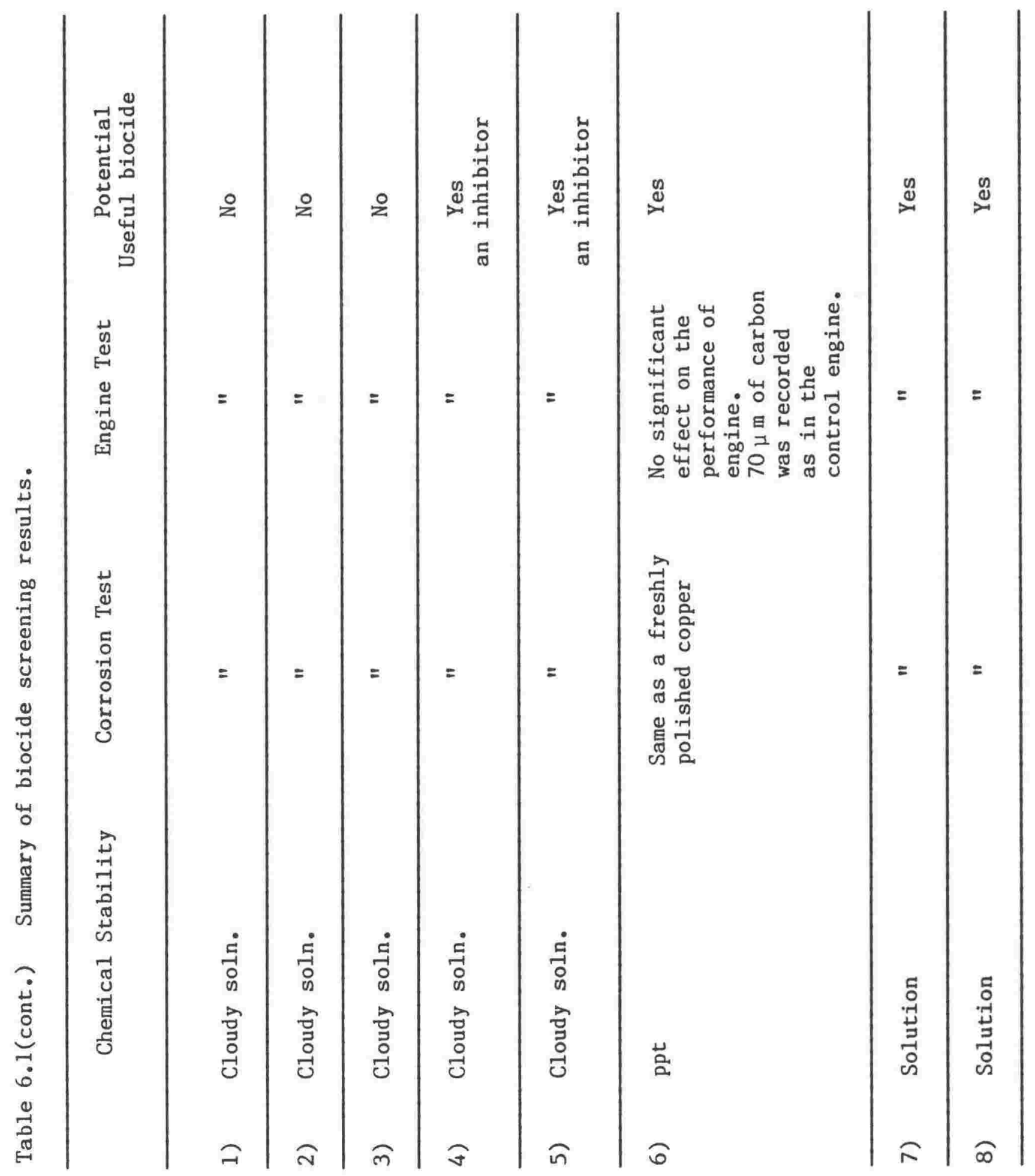


C.R. - Cladosporium resinae

P.C. - Penicillium corylophilum

P.V. - Paecilomyces variotii

(a) The table shows the \% germination and as a superscript (b), an indication of the type or extent of germ tube growth.

1 - Very isolated signs of germtube

2 - Distorted germtubes, very reduced growth, swollen hyphal tips, some bursting.

3 - Unbranched hyphae and no sporulation.

4 - Extensive branching but sporulation rare.

5 - Hyphae elongated, branching sporulation common. 


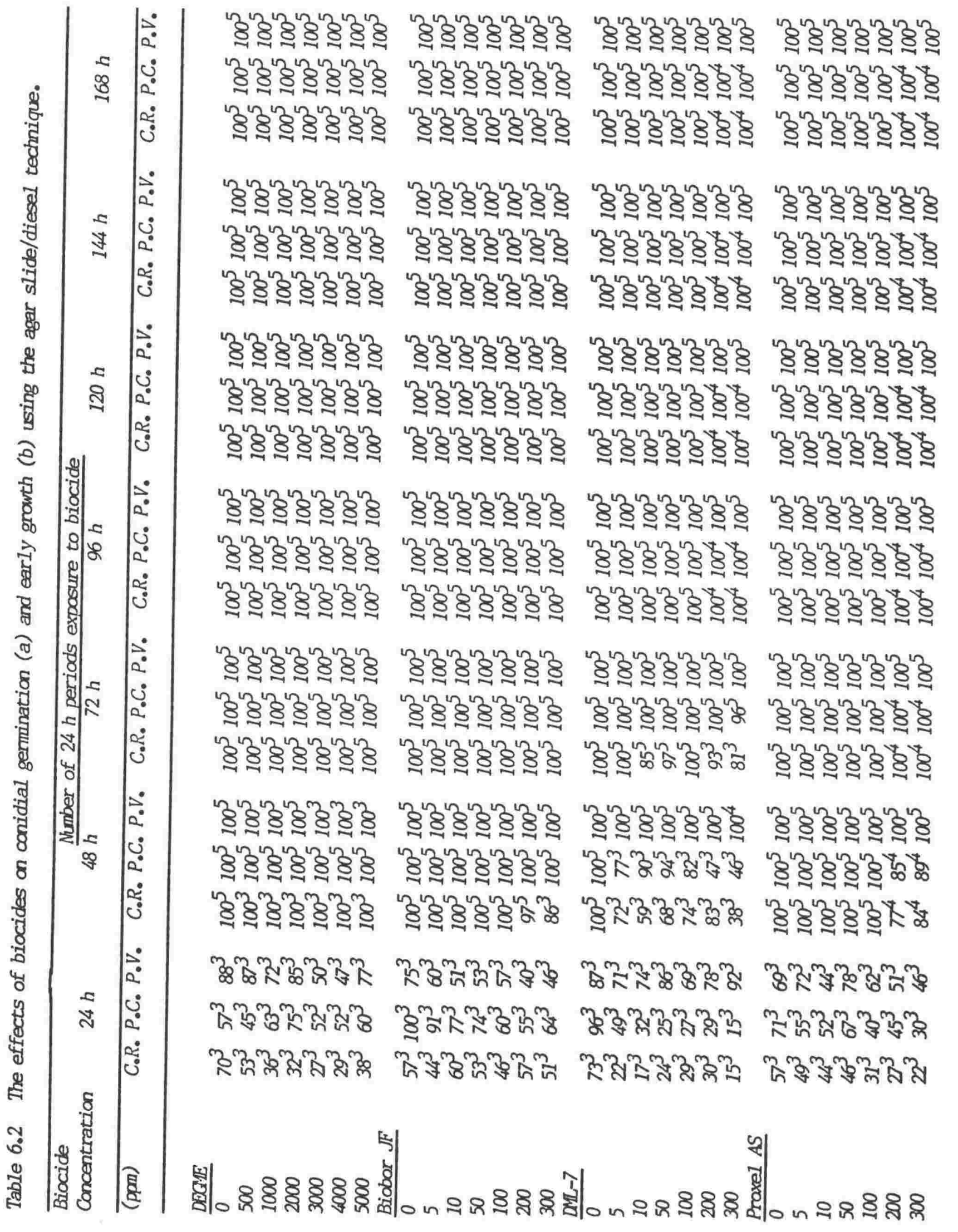




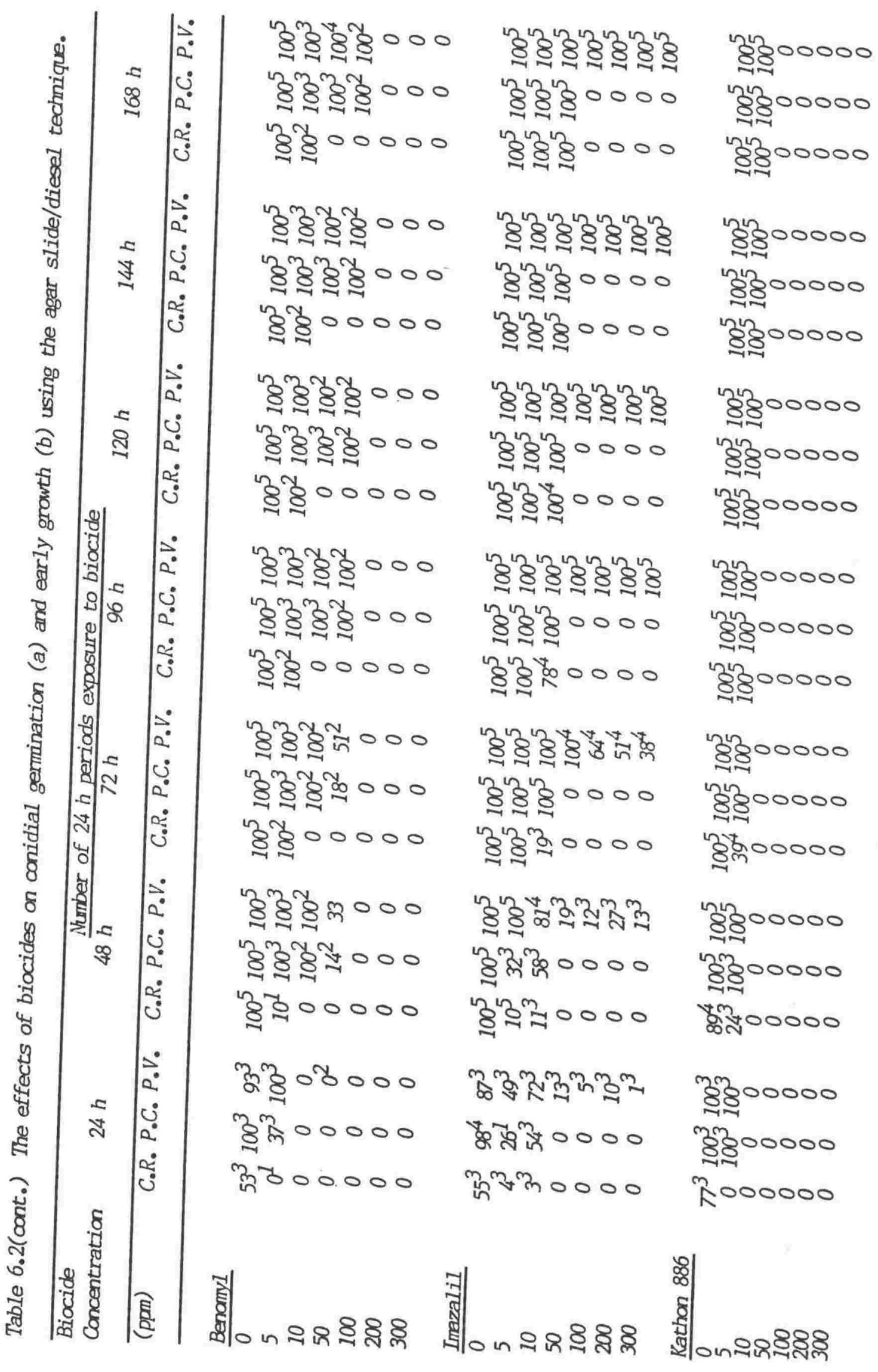


Details of the normal rapid hyphal growth along the interface with frequent branching and sporulation occurring within 48 hours was observed in all control cultures. Hyphae which grew down into the solidified aqueous phase eventually produced conidiophores but conidia were produced profusely on the diesel side of the interwoven interfacial mat. Neither DEGME nor Biobor JF had any effect on this normal growth pattern. In addition to inhibiting germination completely, the effective biocides benomyl, imazalil and Kathon 886 , resulted in the production of deformed germ tubes with limited growth potential. Benomyl for example caused swollen hyphae to form which soon stopped growing and in some cases cell walls and membranes appeared to be disrupted and cell contents leaked out.

With DML-7 and Proxel AS at the higher concentrations, both $C$. resinae and $P$. corylophilum failed to sporulate, indicating that even if these compounds were not biocidal, then some biostatic activity was evident.

\subsubsection{Poison Plate Test}

When agar plugs carrying actively growing mycelia of C. resinae, Paecilomyces variotii, Penicillium corylophilum and the other Penicillium spp. isolated from diesel were placed on MEA the colony diameter increased in a linear fashion to cover the Petri-dish in 8 days, as shown in Figure 6.1. The growth rate was always uniform throughout the 8 days during which colony diameter was measured. Tables $6.3,6.4$ and 6.5 present the growth of these fungi as recorded after 8 days only and show the effects of the inclusion of the various biocides at a range of concentrations upon colony growth rate.

EGME, DEGME and Biobor JF (Table 6.3) had no effect on hyphal growth at any of the concentrations tested under these conditions. Of the other biocides on $1 y$ Kathon 886 inhibited growth of all three fungi more or less equally, being totally inhibitory above $50 \mathrm{ppm}$.

DML-7 and Proxel AS were less inhibitory, having no effect at all on $P$. variotii. Proxel AS was slightly inhibitory to the other fungi at $300 \mathrm{ppm}$ while DML-7 gave some inhibition at $100 \mathrm{ppm}$ affecting some of the fungi more than others. 
Figure 6.1. The growth of treated and untreated fungal colony in the poison plate test. 


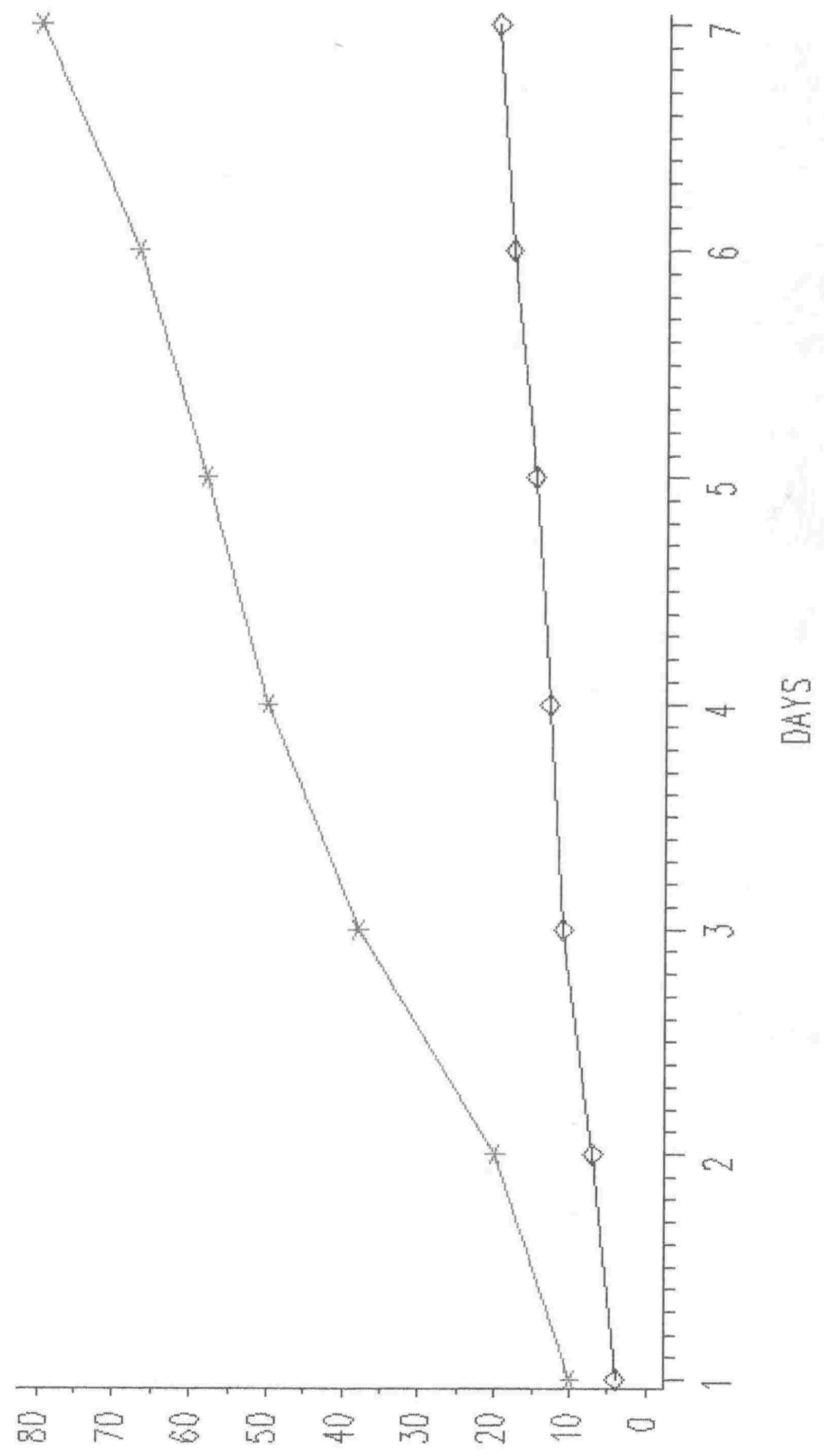

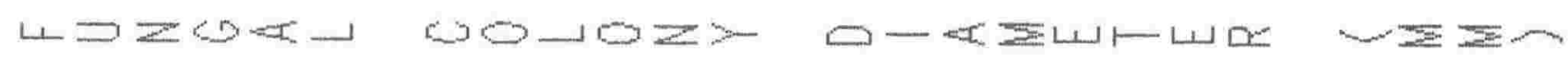


* Growth of fungus from $5 \mathrm{~mm}$ inoculum plug after 8 days recorded as follows :

$0 \quad$ No growth

$+\quad$ Little growth $(6-10 \mathrm{~mm})$

H Moderate growth (11-30 mm)

+++ Profuse growth (31-80 mm)

$* 2 *$

Identical growth responses were obtained from $C$. resinae and $P$. variotii when isolated from diesel fuel which had contained DEGME and Biobor JF for 5 months in the fuel tanks. 


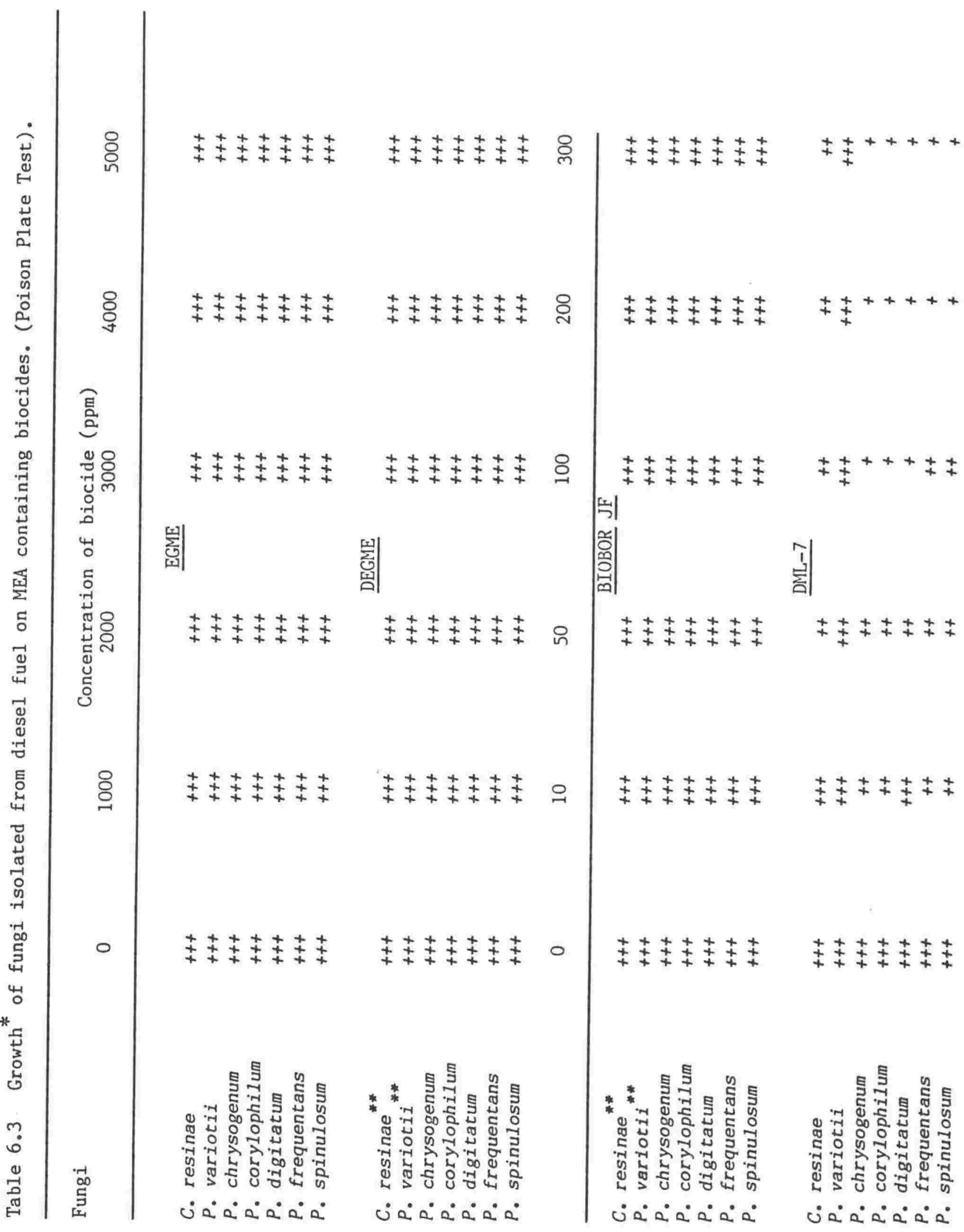




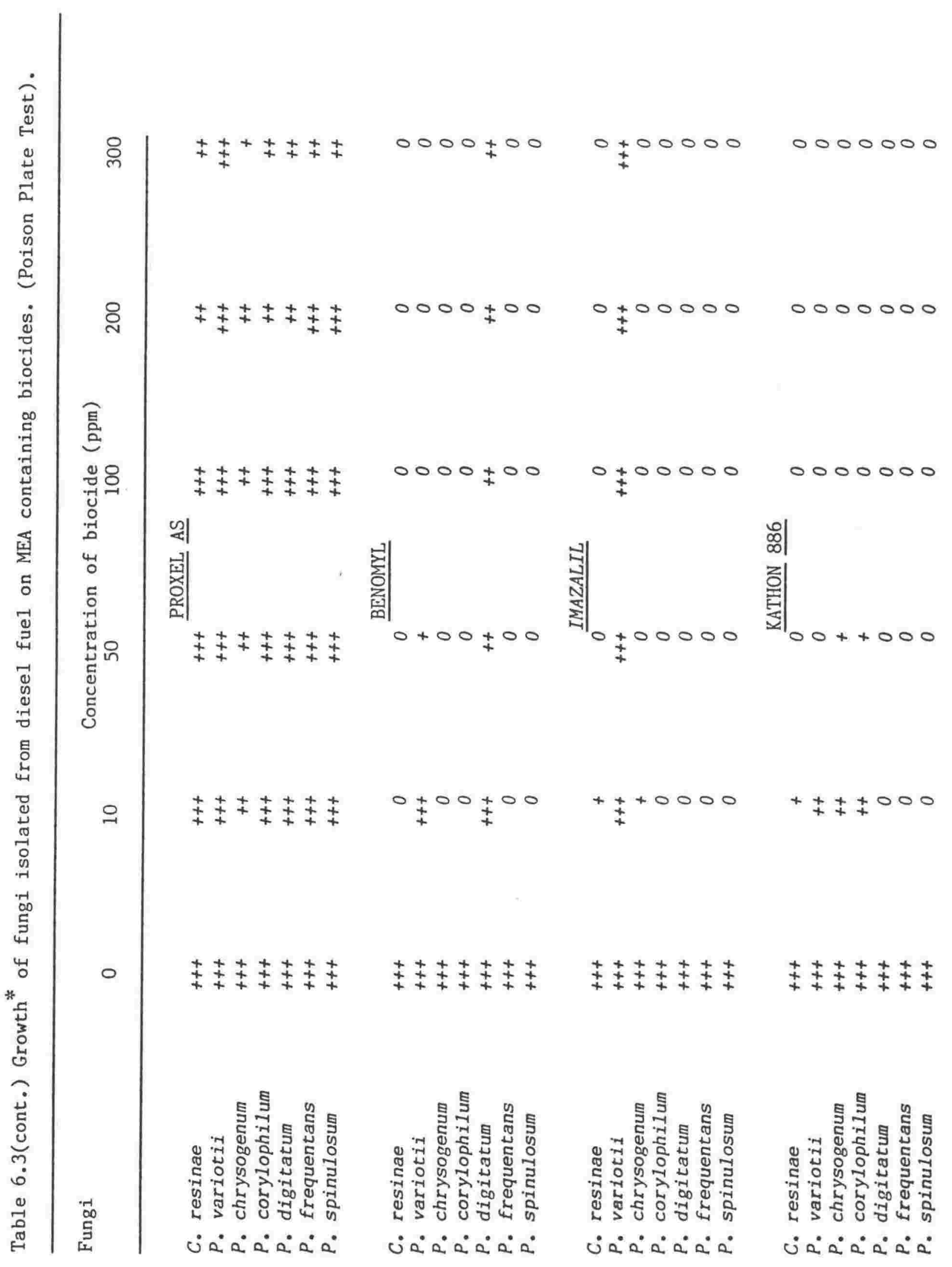




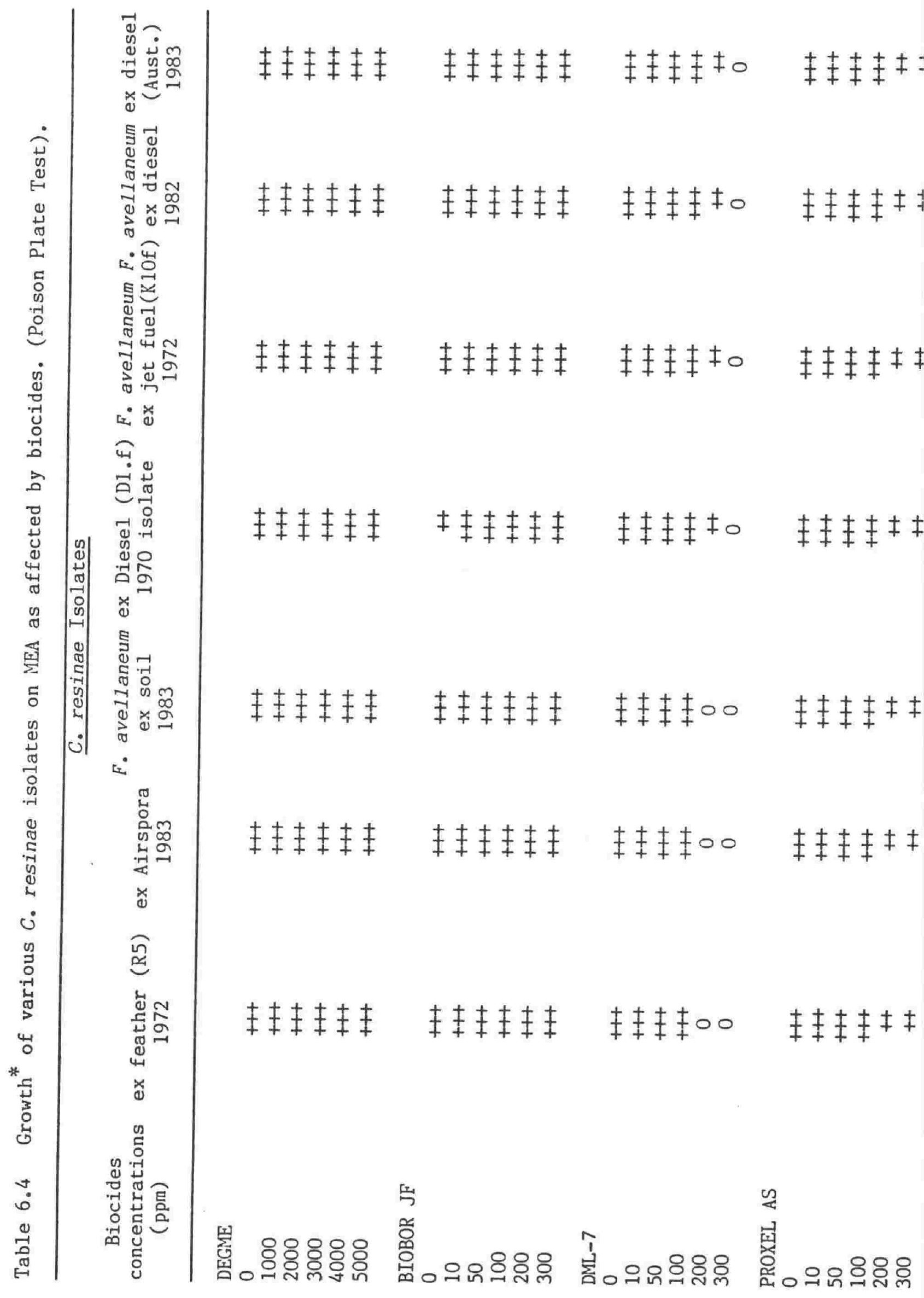




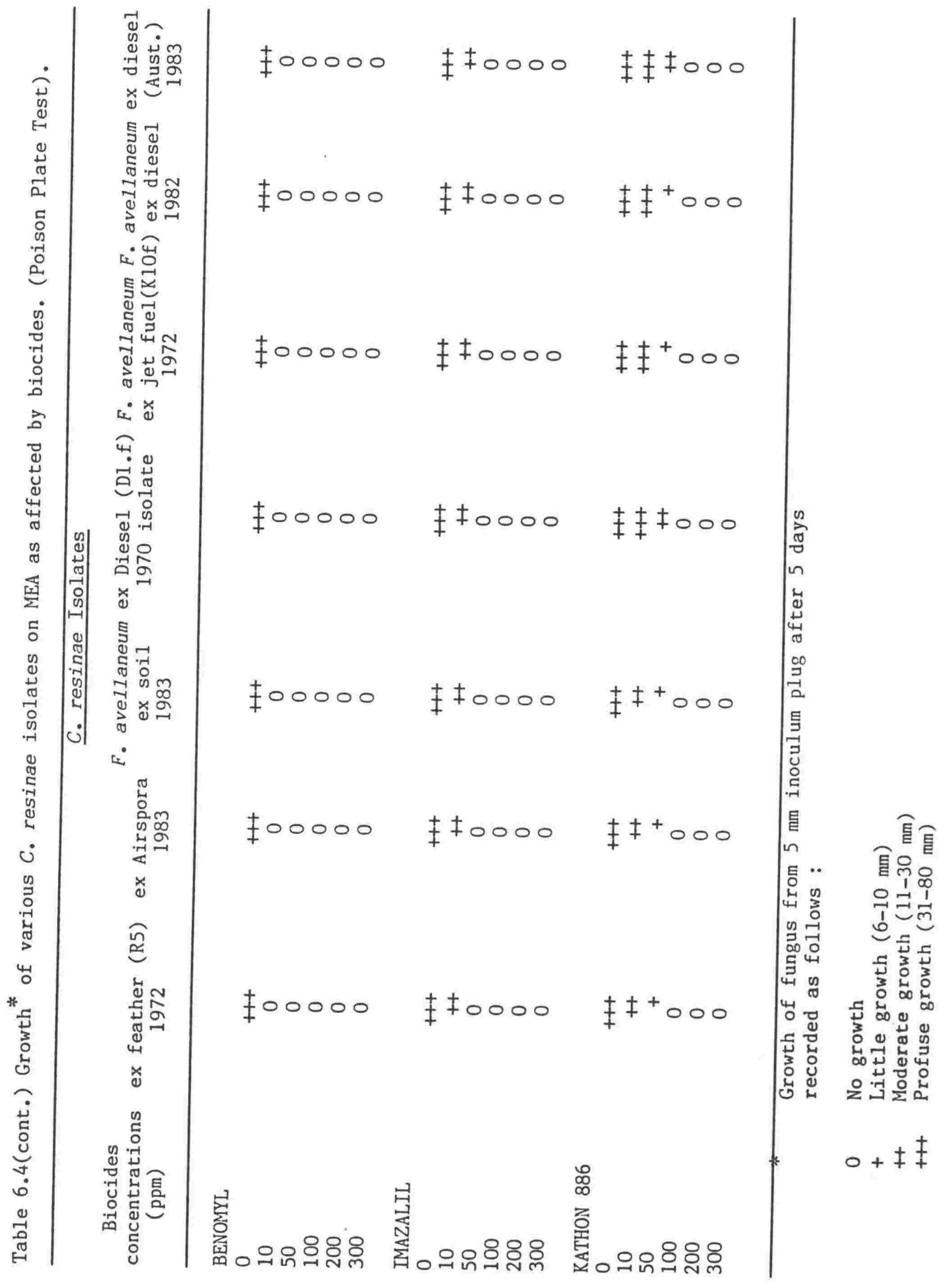




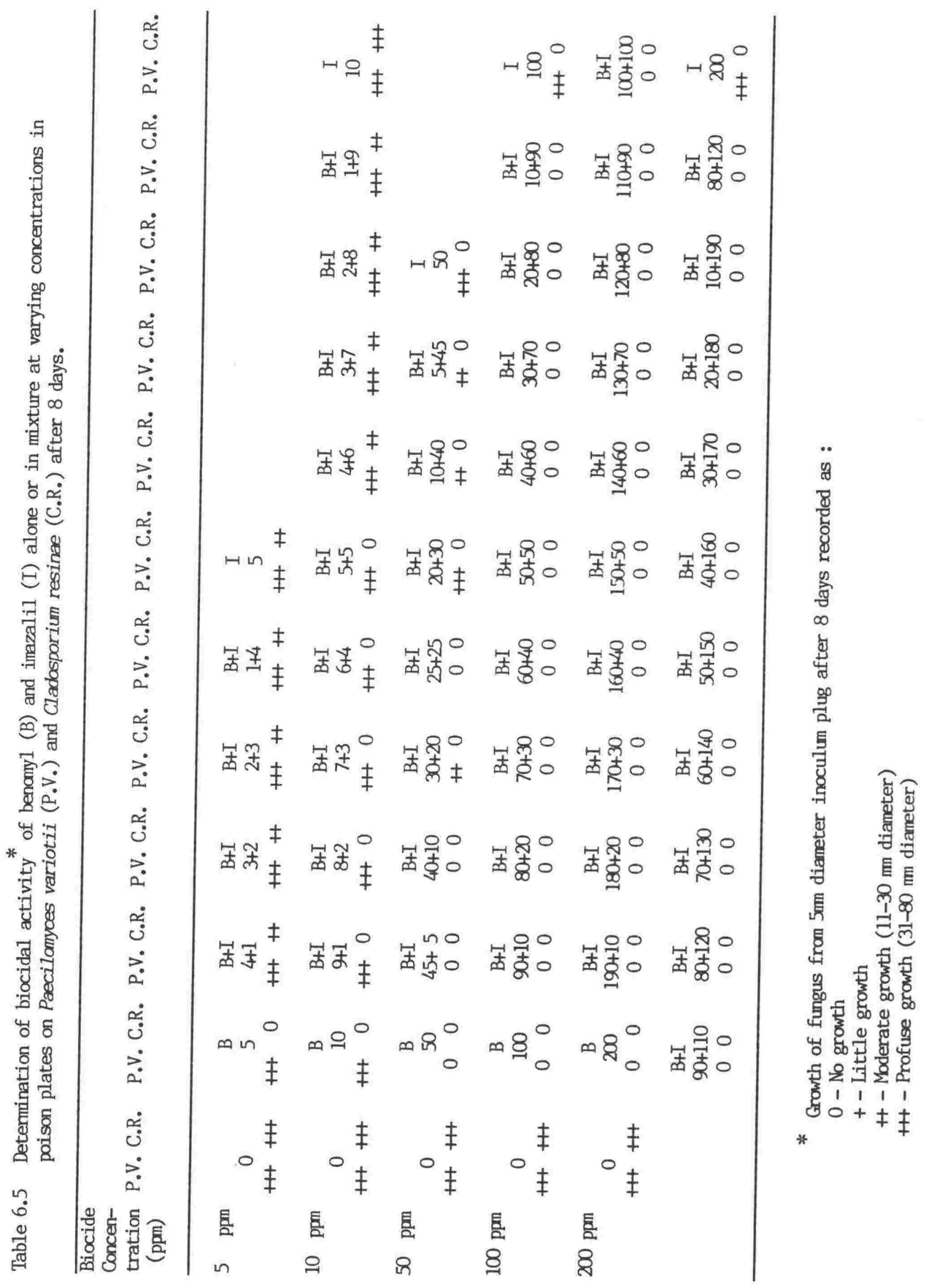


Benomy 1 at $10 \mathrm{ppm}$ caused total inhibition of growth of all fungi

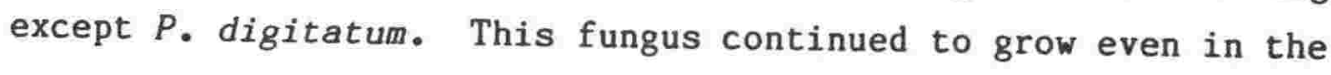
presence of $300 \mathrm{ppm}$ of benomyl.

Imazalil also proved to be very effective, inhibiting the

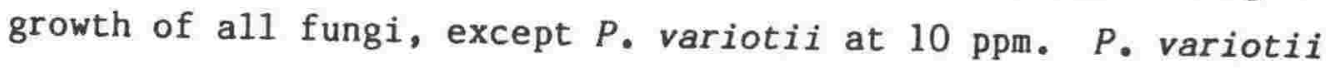
appears to be totally insensitive to this biocide.

Table 6.3 also includes the effects of the biocides on the hyphal growth of $C$. resinae and $P$. variotii isolated from the fuel tanks which had been treated with DEGME and Biobor JF for 5 months. These isolates were insensitive to DEGME and Biobor JF in the laboratory.

The data of Table 6.4 show that the various isolates of C. resinae showed an identical response to the different biocides with all of them including the 1960 and 1970 isolates being insensitive to DEGME and Biobor JF.

The two effective biocides, benomyl and imazalil when tested in combination over a range of concentrations gave the results as shown in table 6.5. At $50 \mathrm{ppm}, C$. resinae showed no growth in any proportion of the two, while $P$. variotii was unaffected by the presence of imazalil and totally controlled by benomyl. When the concentration of benomy 1 fell below $40 \mathrm{ppm}$, the effectiveness of benomyl was partly lost in the presence of the imazalil. Thus it would appear that imazalil somehow reduces the effectiveness of benomyl against $P$. variotii. But at equal proportions of $25 \mathrm{ppm}$, the biocides inhibited both the fungi. Similar interactive effects were apparent at the $200 \mathrm{ppm}$ level where $200 \mathrm{ppm}$ of imazalil was ineffective against $P$. variotii but only $10 \mathrm{ppm}$ of benomyl plus $190 \mathrm{ppm}$ of imazalil gave complete growth suppression. 


\subsubsection{The effects of biocides on fungal growth and survival in $\underline{\mathrm{BH} / \mathrm{diese} 1 \text { phases }}$}

The effects of biocides on spore germination and growth on Bushnell Haas/diesel phases are shown by the results in table 6.6. The most significant effect of the changes in the proportions of the aqueous and hydrocarbon phases were seen at the extremes.

In the absence of biocides, the mixed spore inoculum failed to grow on either the aqueous phase or on diesel alone. The changing proportions of the two phases in the mixtures had some effect on the amount of fungal growth. It was evident that greatest growth was obtained in liquid media containing almost equal volumes of the two phases and least when one phase was $20 \%$ of the total volume. Once again DEGME and Biobor JF had no significant effect on fungal growth. Both DML-7 and Proxel AS caused a significant reduction in fungal growth with the effect being the same at all three concentrations. No systematic variation in the degree of inhibition accompanied the changing proportions of aqueous/hydrocarbon phases. Both benomyl and imazalil almost completely prevented any fungal growth at all concentrations of biocide and in all proportions of Bushnell-Haas medium and diesel. When little growth was observed in the presence of DML-7 and Proxel AS, spores retained their viability when filtered out and placed on V-8 juice agar medium. After 6 weeks in medium containing benomyl at or above $50 \mathrm{ppm}$, all spores had lost their viability except for Penicillium digitatum. In the presence of imazalil, at or above $200 \mathrm{ppm}$, all fungal spores lost viability except for $P_{\text {. variotii. }}$

The $\mathrm{pH}$ in all the test systems was between 5 and 7 at the end of 6 weeks. The aqueous phase in Proxel AS bottles appeared clear but all the others were cloudy. There was a deposit at the bottom of benomyl treated bottles.

A fresh batch of DEGME gave identical results under these test conditions and remained ineffective. 
Mixed spore inoculum was used in this test.

** Growth recorded after 6 weeks as follows:

\section{No growth}

+ Some spore germination, very little growth

++ Very thin colourless mycelial mat

++ Thick dark mycelial mat

And as + Dry weight (mg) of fungal mycelia

The data in the ( ) is from the new batch of DEGME tested

Spore viability results:-

Benomyl completely controlled $C$. resinae and P. variotii at $50 \mathrm{ppm}$, and Penicillium digitatum survived but did not form a mycelial mat.

Imazalil controlled $C$. resinae and Penicillium spp. at 100 ppm but not Paecilomyces variotii. DML-7 and Proxel AS did not kill the spores at any concentration but inhibited growth. 


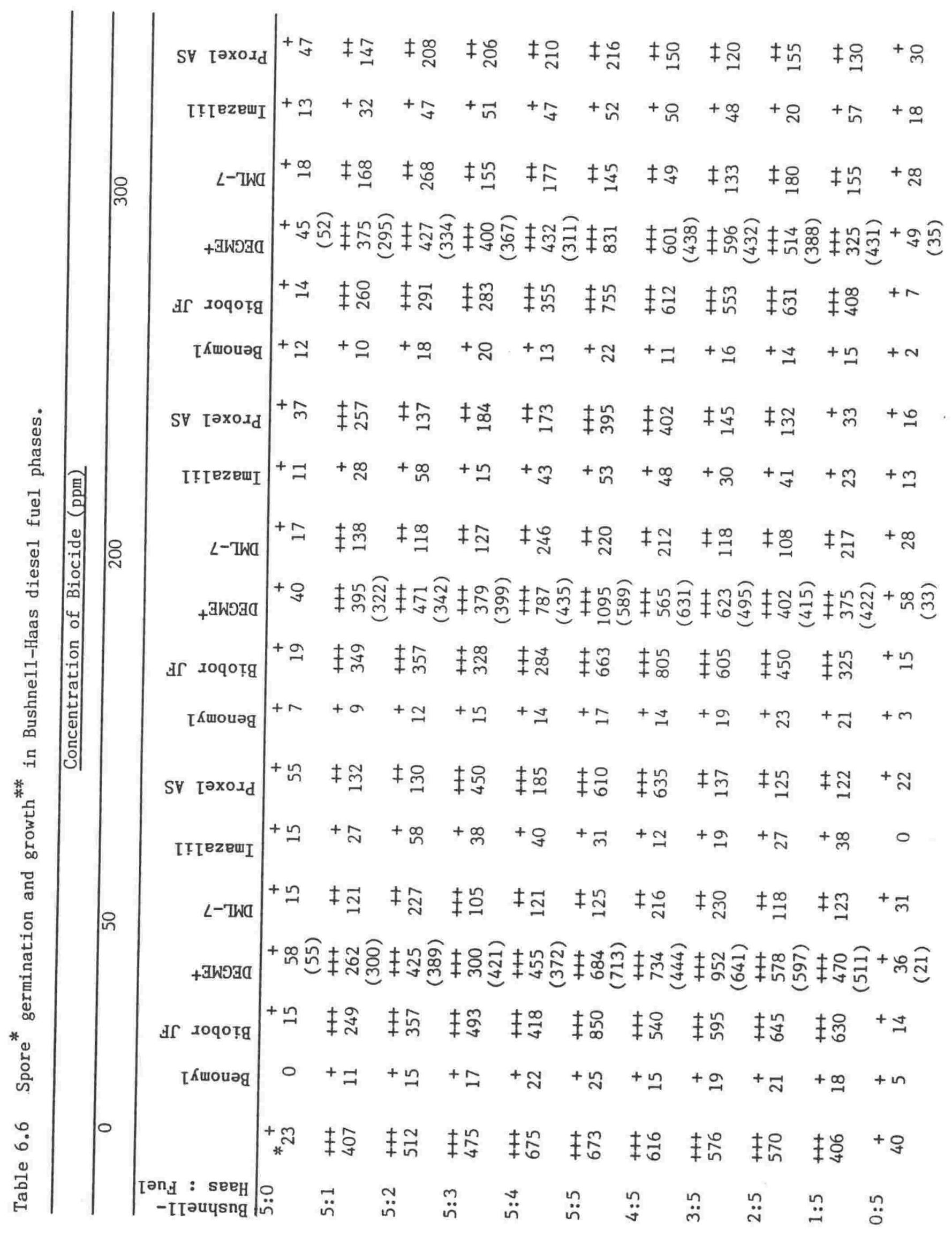


When a $C$. resinae spore suspension was inoculated into $\mathrm{BH} /$ diesel mixture, held at $4^{\circ} \mathrm{C}$ for 4 weeks and then returned to normal temperature, growth was reduced at the low temperature and was then vigorous at $18-19^{\circ} \mathrm{C}$. Even after 4 weeks exposure to DEGME and EGME under slow growth conditions these biocides had no effect on subsequent growth.

\subsubsection{Large volume liquid fuel : biocide tests}

Addition of biocides to two litre bottles of diesel fuel taken directly from the storage tanks gave results similar to the other laboratory growth studies (table 6.7).

The number of CFU of $C$. resinae, $P$. variotii and Penicillium spp. showed some variation in the different two litre batches before addition of the biocides. Penicillium spp. occurred at a lower frequency compared to $C$. resinae and $P$. variotii.

Kathon 886 was biocidal after 2 days exposure at $300 \mathrm{ppm}$ and after 3 days at 100 and $200 \mathrm{ppm}$ to all the fungi. C. resinae was inhibited at $50 \mathrm{ppm}$ after 6 days. $P$. variotii was slightly more tolerant being almost unaffected at $50 \mathrm{ppm}$.

Both benomyl and imazalil were effective against $C$. resinae but some CFU of Penicillium spp. managed to survive at $300 \mathrm{ppm}$ after 5-6 days exposure to benomyl. Imazalil was ineffective against $P$. variotii but benomyl gave good control at all concentrations.

\subsubsection{Intermittent exposure of the three common fuel fungi to biocides in diesel fuel}

Intermittent exposure to biocides of a mixed spore proportion carried on sterile filter papers in diesel fuel for 24 hour periods separated by 6 day exposure in clean diesel fuel gave results shown in table 6.8. Biobor JF was biocidal to Penicillium corylophilum at 50 ppm after three 24 hour exposures but had no effect on $C$. resinae and $P$. variotii. DML-7 was effective against $C$. resinae after five 24 hour periods at $100 \mathrm{ppm}$ but reduced viability almost to zero after two exposures at 200 and $300 \mathrm{ppm}$ of all three fungi. 
$x$ C.r. - C. resinae

Pen. - Penicillium spp.

P.v. - Paecilomyces variotii

* Two 1itre samples were incubated with biocides at various concentrations

+ Numbers of colonies per litre are calculated from colonies counted after the fungi have been allowed to form colonies on V-8 juice agar plates for 5 days. 


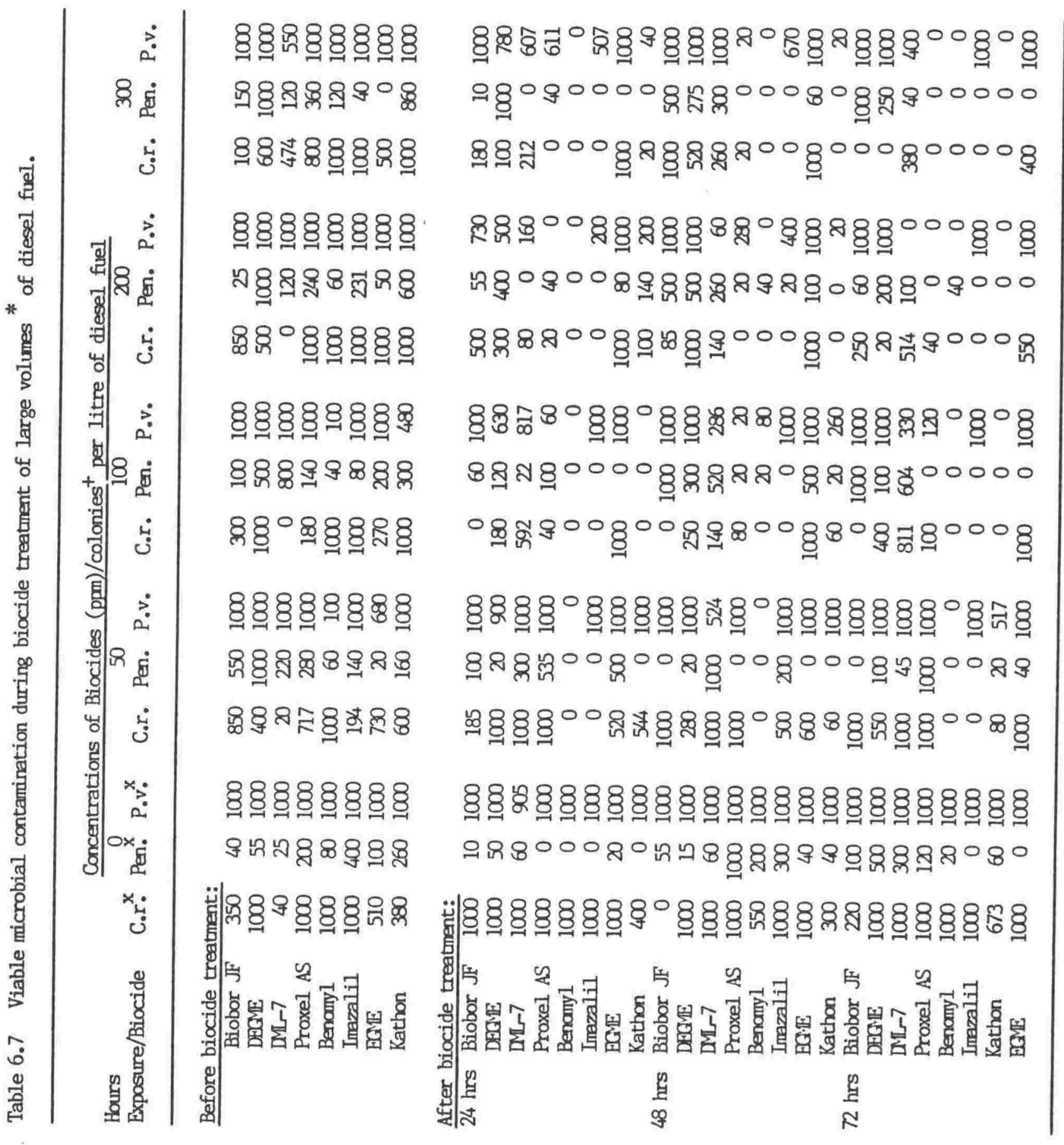




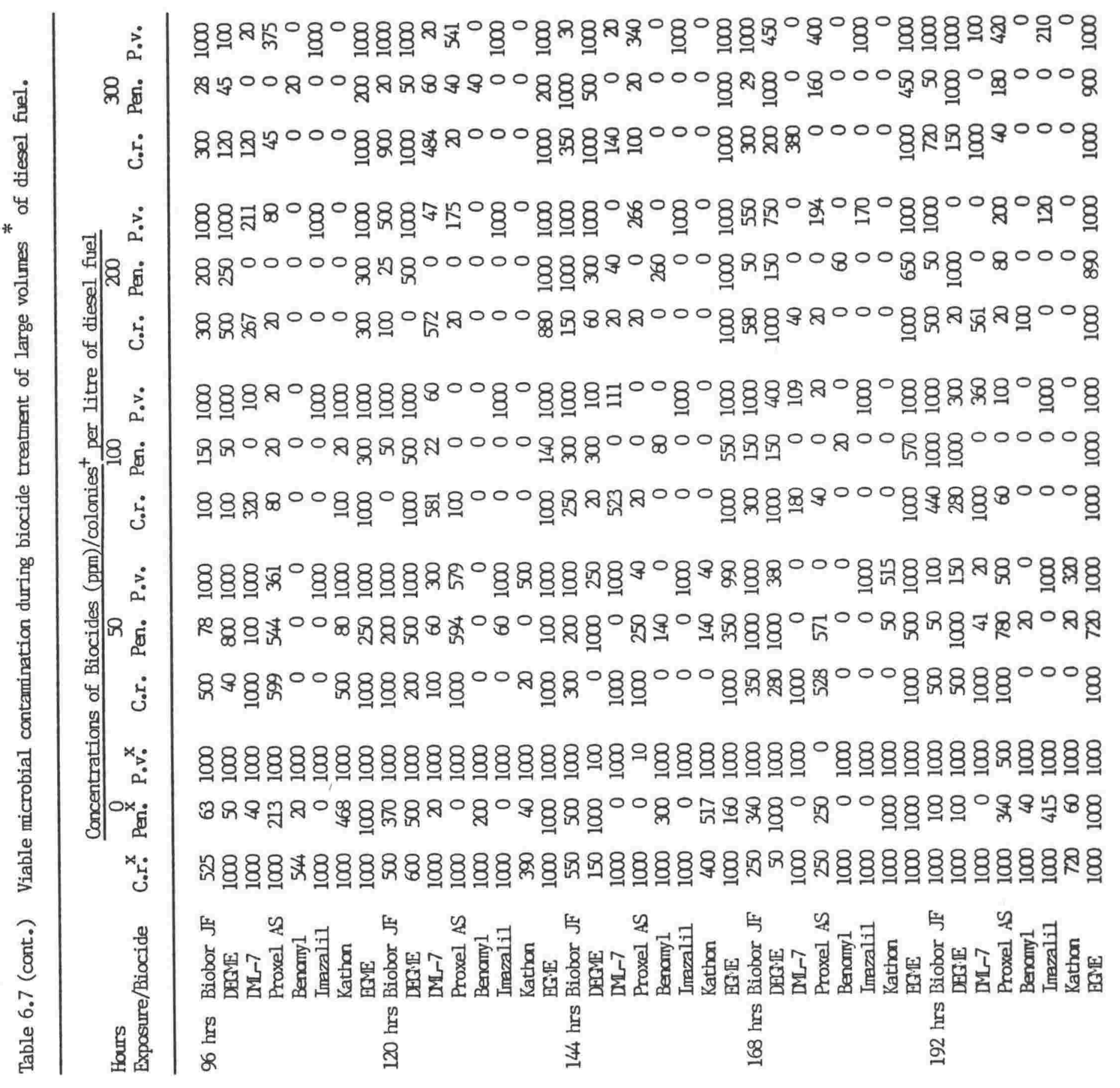


${ }^{\mathrm{X}}$ C.R. - Cladosporium resinae

P.C. - Penicillium corylophilum

P.V. - Paecilomyces variotti

* Figures shown are the total CFU score from 3 replicate filters counted after 5 days growth in V-8 juice agar.

+ Moist sterile filters carrying spores were exposed to diesel containing biocide for 24 hours followed by exposure to sterile diesel for 6 days before repeating up to 7 times, some filters were removed for determining viable CFU after each treatment.

0 - no growth or no colonies

$1->10$ colonies/filter

2 - > 20 colonies/filter

3 - > 30 colonies/filter

4 - > 40 colonies/filter

5 - > 50 colonies/filter 


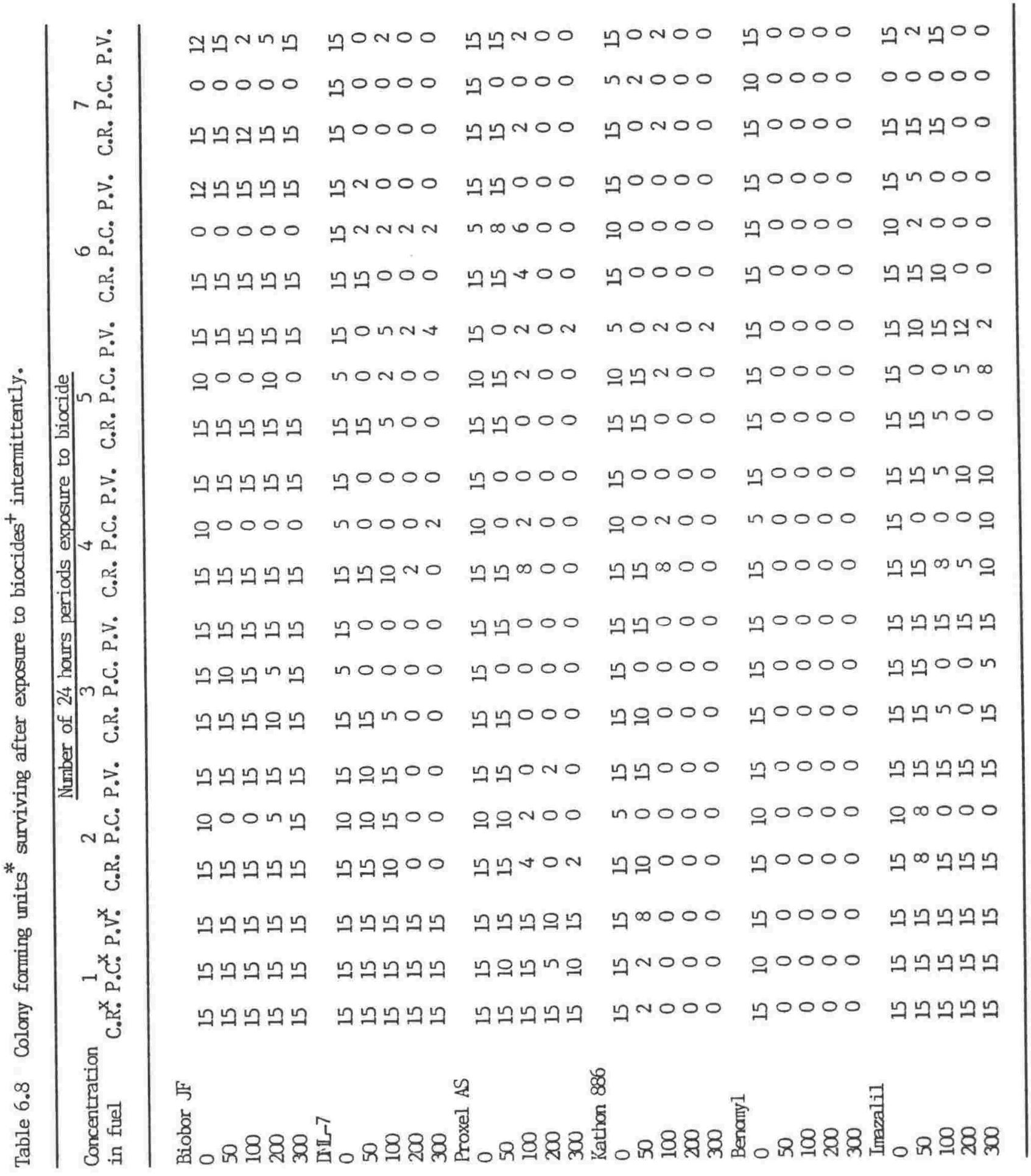


Kathon 886 required three 24 hour exposures at 50 ppm but was biocidal to all three fungi after only one 24 hour exposure at $100 \mathrm{ppm}$. Benomyl was effective after a single 24 hour exposure, killing all fungi. Imazalil was effective against $C$. resinae after five 24 hour exposures and required $\operatorname{six} 24$ hour exposures to inhibit $P$. variotii and Penicillium corylophilum.

\subsection{SECTION II - Field studies of biocide effects}

\subsubsection{Effects of biocides on microbiological contamination.}

The effects of biocides added to field storage tanks on microbiological contamination, corrosion and engine performance are reported here.

Table 6.9 presents the mean number of CFU isolated from the DEGME and Biobor JF treated and untreated tanks. Complete data on the effects of DEGME and Biobor. JF at the different levels of the tanks in the field trials are given in Appendix 6.1 .

DEGME and Biobor JF significantly reduced $C$. resinae but had little effect on Paecilomyces variotii and Penicillium spp. Numbers of P. variotil, Penicillium spp. and Trichoderma spp. were intermittently high in all the tanks. $P$. variotii was observed to peak only in late June, July and August 1984. The decrease of Penicillium spp. and Trichoderma sp. seemed to alternate with increase

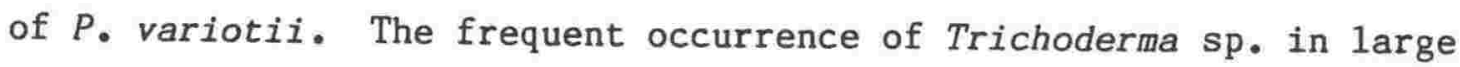
numbers and a decrease in all fungi in early August suggest a seasonal influence. It was also notable in April that whenever a particular fungus occurred in high numbers, it remained high in all the sampling levels. The numbers fluctuated at the different levels of the tank only when the fungus occurred in small numbers.

Table 6.10 shows the distribution of DEGME, Biobor JF, DML-7 and Proxel AS mainly in the fuel phase. When excessive amounts of water were present on the very bottom of the tank, Proxel AS partitioned preferentially into the water phase. DEGME and Biobor JF were water soluble and as the water content increased they partitioned into the water phase. 


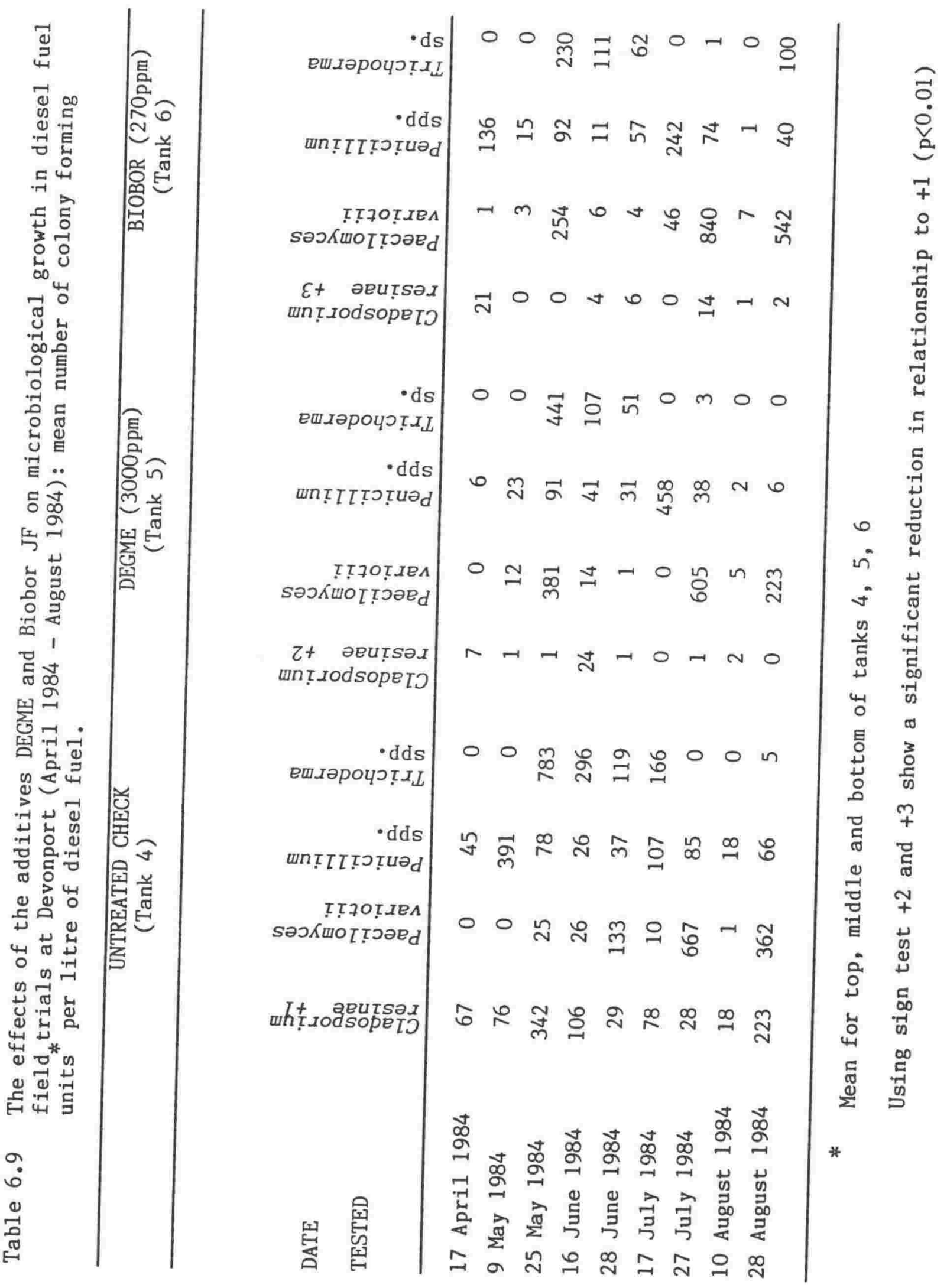




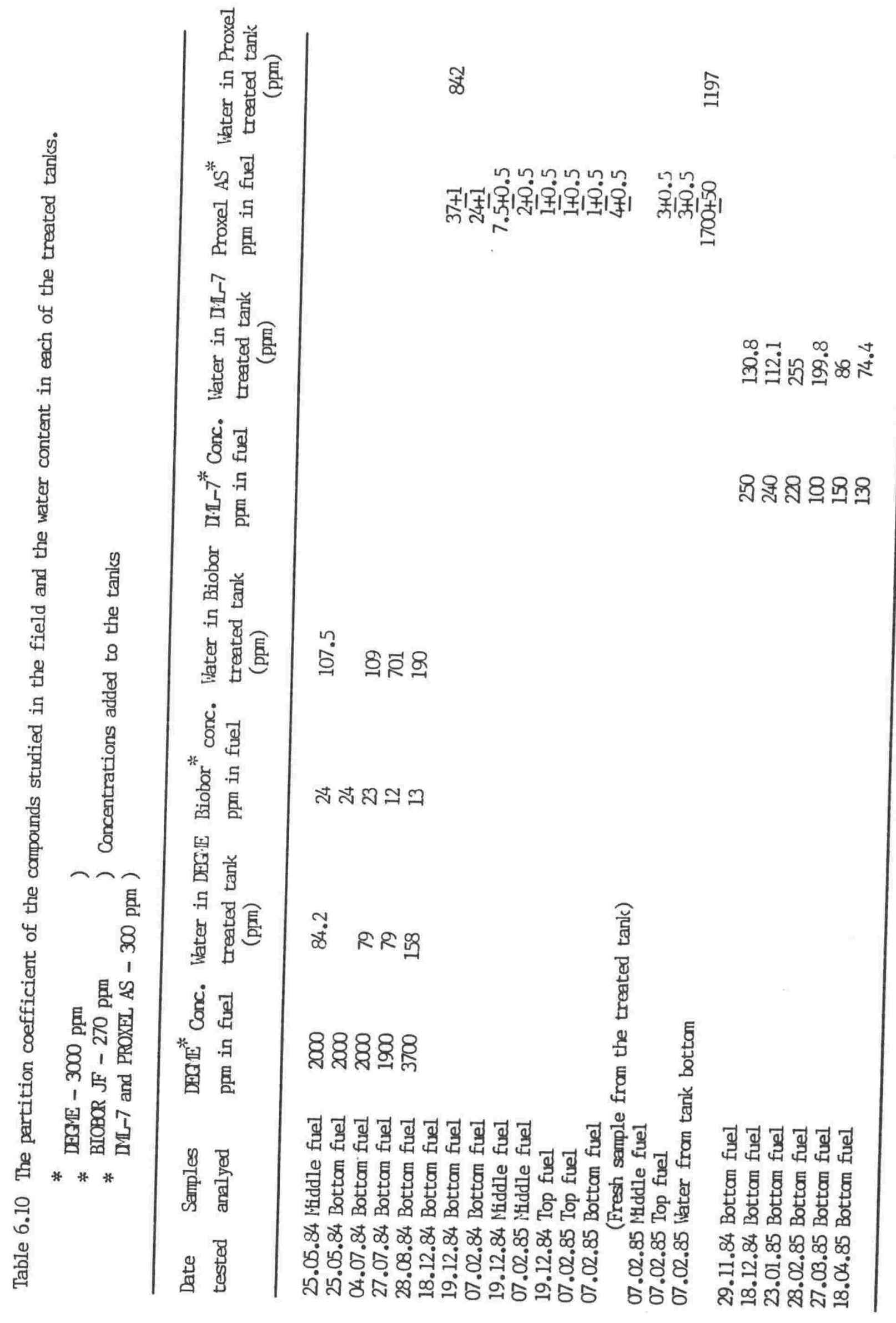


Although $270 \mathrm{ppm}$ of Biobor JF was added into the fuel, only 13-24 ppm remained in the fuel phase throughout the five months of sampling. But $C$. resinae numbers remained low in the treated tanks, regardless of the amount of water present in the tanks. The total water content fluctuated in both the untreated and treated tanks.

The data in table 6.11 show the effect of DML-7 and Proxel AS on microbiological growths in field tanks. Throughout the sampling period the untreated tank remained highly contaminated with $C$. resinae and Penicillium spp. $P$. variotii contamination fluctuated. There was an increase in the different species of fungi in tank 4 compared to the treated tanks (Appendix 6.7). In late November 1984 in the untreated tank, the presence of high numbers of $P_{\text {. variotii }}$ corresponded with a decrease in numbers of $C_{\text {. }}$ resinae and Penicillium spp. In December the decrease of $P_{\text {. variotii corresponded with high }}$ numbers of $C$. resinae and Penicillium spp. Probably, interaction occurred between the predominant fungi in the tanks, whereby each fungus was not able to tolerate the presence of another. The water bottom of the tanks was free from microbiological contamination in the presence of the high proportion of DML-7 and Proxel AS. In February and March 1985 high numbers of C. resinae occurred in tank 2 (containing DML-7) and in the untreated tank, suggesting Proxel AS to be more effective than DML-7. Generally both the compounds significantly inhibited $C$. resinae and Penicillium spp. in relation to the untreated tank. The numbers of the predominant fungi fluctuated

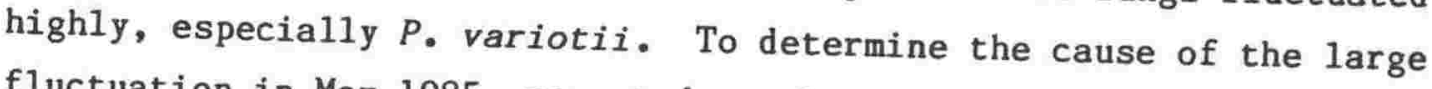
fluctuation in May 1985, repeated samples were taken from each level in each tank (Appendix 6.3). It was found that when C. resinae,

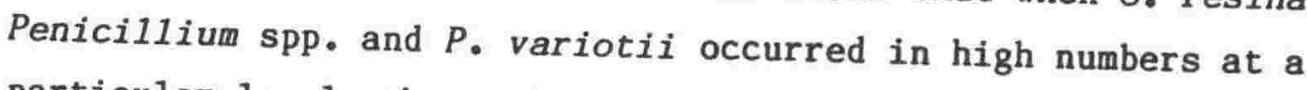
particular level, the number of CFU fluctuated between replicates. The presence of Mucor, Rhizopus and the other fungi listed in Appendix 6.2 were casual and occurred infrequently in high numbers.

Ninety-nine percent of the Proxel AS migrated to the water phase in the storage tank (table 6.10). A layer of water bottom was observed in the Proxel AS treated tank. The $\mathrm{pH}$ of the aqueous phase was between 5 and 6.5 throughout the trial period. Proxel AS was also unevenly distributed through the diesel in the storage tank. 


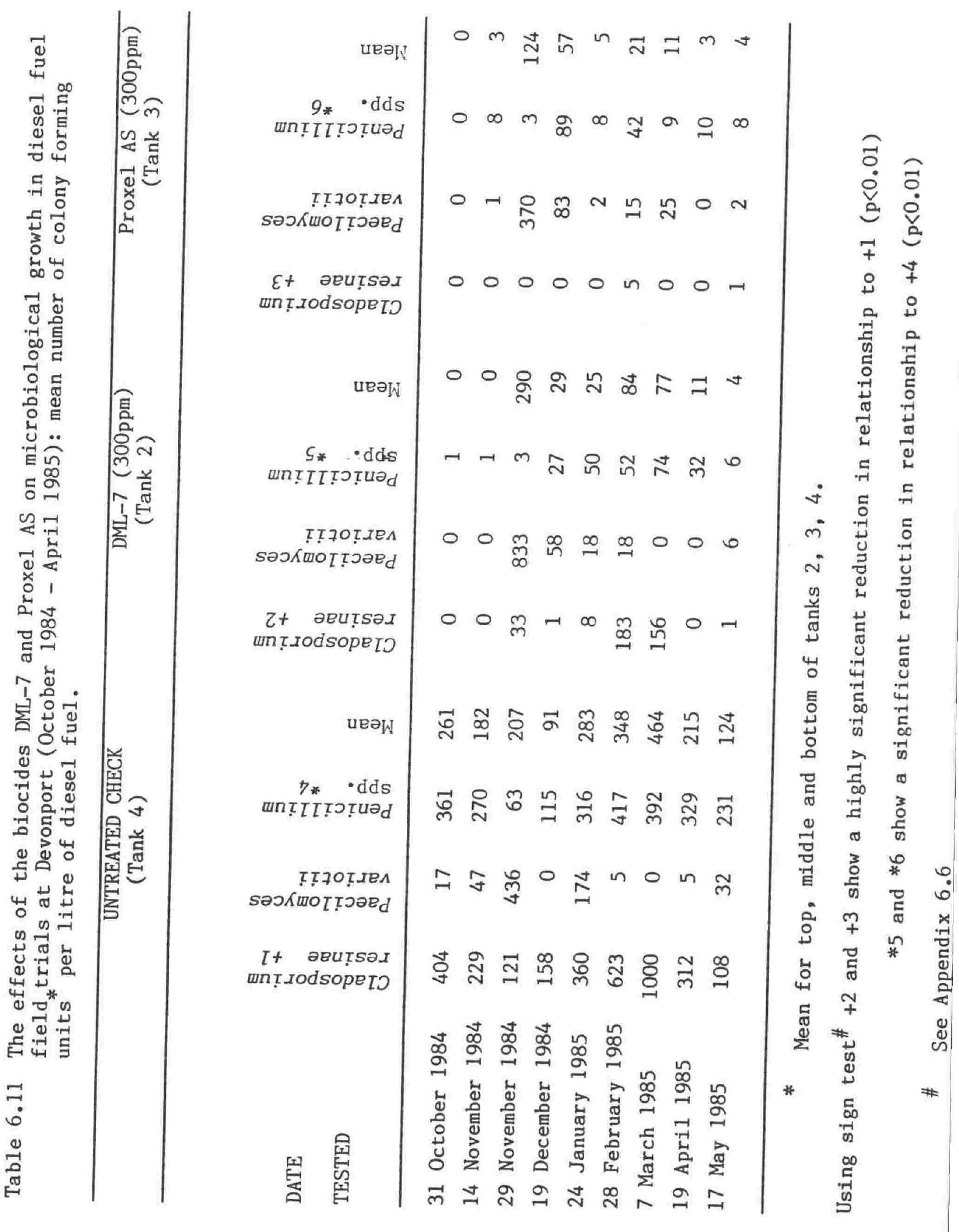


It was unstable in diesel and may have undergone oxidation by dissolved air. It was also evident from the table 6.9 that when Proxel AS was analysed in the same sample of fuel repeatedly at intervals in the laboratory, it was observed to partition continually into the aqueous phase.

The methylene bis thiocyanate, an active ingredient in DML-7, was found to be water soluble and migrated rapidly to the aqueous phase on vigorous shaking. Analyses carried out in the laboratory have demonstrated that in 1:250 water/diesel fuel system, stirred gently, DML-7 at $300 \mathrm{ppm}$ in the fuel produced a level of methylene bis thiocyanate equivalent to $76 \% \mathrm{DML}-7$ in the water phase after 4 hours at room temperature. The rate at which the chemical compounds partition from fuel to water will clearly be a factor of great consequence to the treatment of fuel storage tanks, where agitation to increase the partition rate may be impracticable.

It was observed that imazalil and benomyl significantly controlled all the predominant fungi (table 6.12). The untreated tank was highly contaminated by $C$. resinae and Penicillium spp. However the $P$. variotii CFU occurred in low numbers and fluctuated in all three tanks. (In the laboratory, imazalil was not effective against $P$. variotii, but inhibited it in the field). Increased numbers of fungal species were isolated from all the three tanks (Appendix 6.4). The majority occurred in small numbers. Zygorhynchus sp. occurred infrequently in high numbers in all the tanks. The CFU between replicates did not show much variation (Appendix 6.5). The total water content in the untreated and treated tanks fluctuated slightly and remained below $115 \mathrm{ppm}$ throughout the trial (Appendix 6.4). As benomyl and imazalil were more fuel than water soluble, they may partition slowly and decompose into the aqueous phase, maintaining an effective biocidal activity at water/diesel interface. 


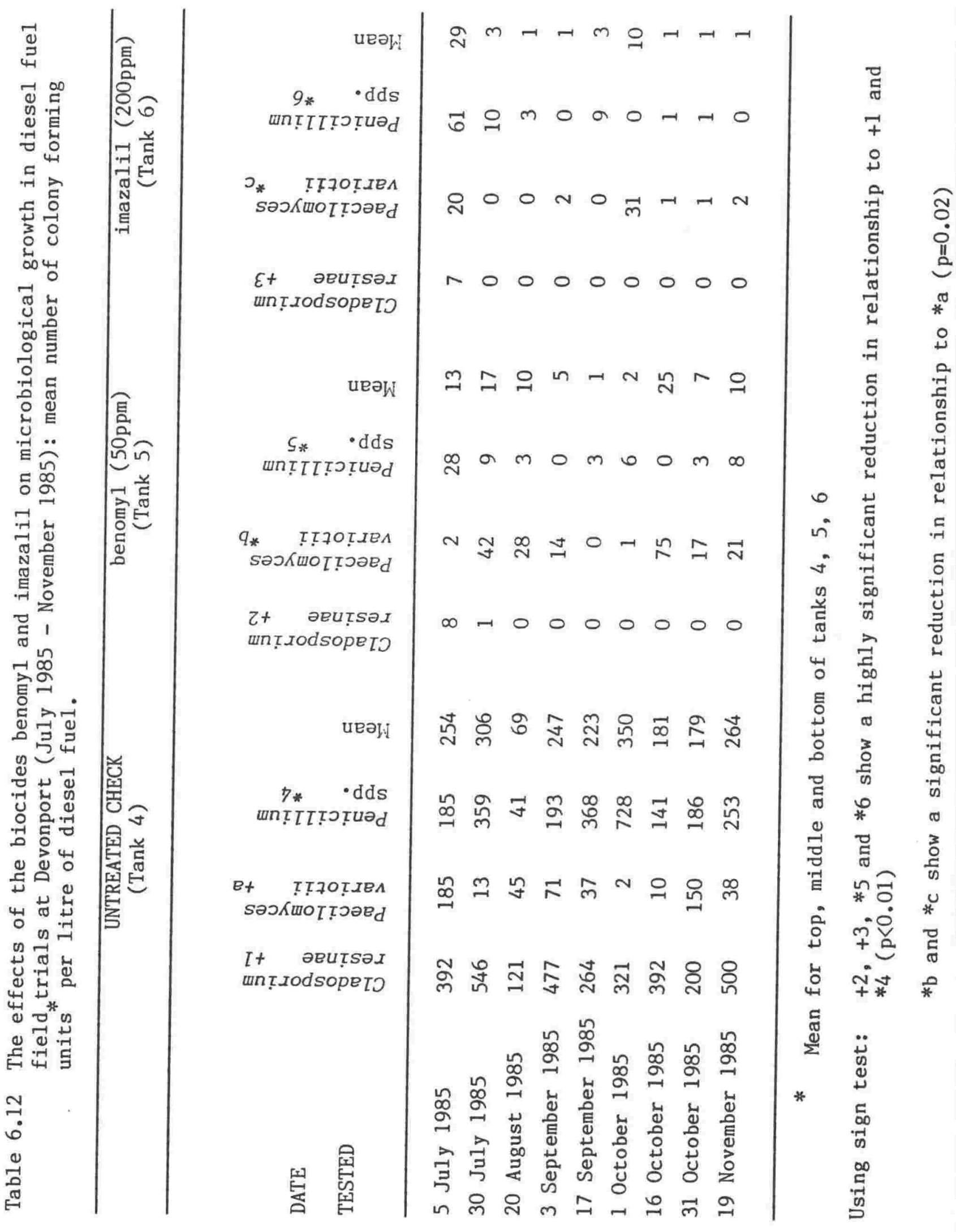




\subsubsection{Test on engine performance}

In the engine trial test, none of the compounds had a significant effect on the performance characteristics of the diesel fuel from thermodynamic aspects. Refer Appendix 6.7 for graphs on diesel engine additive trials.

The carbon deposit (ash residue) was measured in the form of black sooty deposit formed on the piston as in Figure 6.2. A depth of $70 \mu \mathrm{m}$ of carbon was recorded when the treated and untreated fuel was used in the engine for all the compounds tested.

\subsubsection{Corrosion test}

In accordance with the IP154/84 corrosion test method, no significant corrosion was recorded on the copper strip for all the compounds. The test compounds proved to be "la", almost the same as a freshly polished copper strip. 


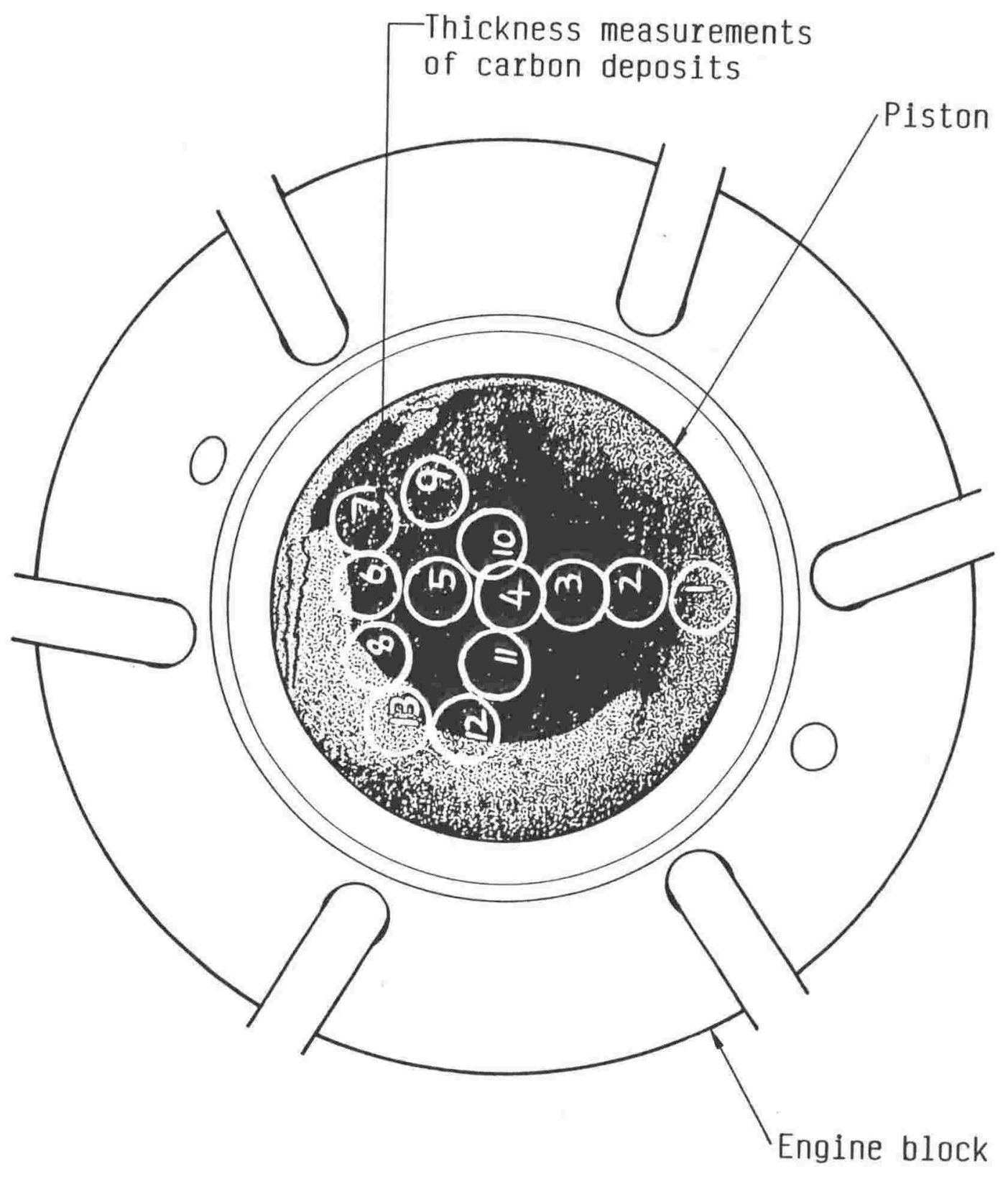




\subsection{Discussion}

Although $C$. resinae is generally considered to be the most troublesome contaminant of jet and diesel fuel (Hendey, 1964; Berner and Ahearn, 1977), Paecilomyces variotii and Penicillium spp. can also produce mycelial growths. In this study all the fungi were examined in biocide tests.

Most of the earlier workers (Klemme and Leonard, 1960; Elphick and Hunter, 1968; Rogers and Kaplan, 1968, Hendey et a1, 1971; Neihof and Bailey, 1978) and the recent work by Smith and Crook (1983) have considered the effects of biocides on $C$. resinae alone.

IEGME, EGME and Biobor JF were included in this work because they were the only known commercial products widely used in the fuel industry. Ethylene glycol alkyl ethers have been known for many years to be bacteriacidal (Berry and Michaels, 1950) and EGME is the form that has become widely accepted for application to jet fuels and it is now commonly used. Neihof and Bailey (1978) reported that of the seven compounds tested, diethylene glycol monomethyl ether (DEGME) was the most suitable replacement since it was effective at $1-2 \%$ (in aqueous phase) compared with 10-17\% required for EGME. However, most of the other compounds tested had less favourable partition coefficients than EGME and it would be more difficult to ensure that the required anti-microbial concentration accumulated in the water phase. Despite their short-comings, in particular their ability to stimulate growth at low concentration, the ethylene glycol derivatives were widely accepted as they combust completely, are freely fuel miscible, and are readily available.

The other biocidal compounds in wide use since the early 1960s are the organoborates. Borates have long been known to inhibit microbial and enzymic activity (Zittle, 1951) and this activity was found to be greater for organic borate compounds. The activity of organoborates has been investigated by DeGray and Fitzgibbons (1966). Some organoborate compounds were known to precipitate boric acid when treated fuel comes into contact with water and this may cause filter plugging. 
One product which is widely used, Biobor JF, was found by Saunders, Wotring and Taylor (1966) not to undergo this precipitation. The recommended concentration for use of Biobor JF was between $135 \mathrm{ppm}$ for continuous use and $270 \mathrm{ppm}$ for shock treatment. Later it became evident that the anti-microbial activity of both EGME and Biobor JF was quite slow, a long exposure time being required for effective use and these compounds were found to stimulate microbial growth at low temperature (Genner and Hill, 1981) in addition to low concentrations.

In the present studies, laboratory tests showed that DEGME, EGME and Biobor JF were ineffective against all the predominant fungi at room temperature. Tests on agar plates showed no effect on hyphal growth. Effectiveness in the presence of diesel was studied and an attempt made to simulate the field conditions. Tests carried out on the agar/diesel culture confirmed that they were ineffective as they were in the $\mathrm{BH} /$ diesel medium and in the large volume liquid fuel.

The restriction of extensive hyphal growth along the water/diesel interface observed in the DEGME, EGME and Biobor JF treated and untreated agar slide culture in this study may be a nutritional effect (Smith and Crook, 1980) and may also be due to the high solubility of oxygen in the fuel (Teh and Lee, 1973). Hill, Evans and Davies (1967) have determined experimentally that kerosene in equilibrium with air would in conditions of modest turbulence pass oxygen to an aqueous bottom at a rate and to a level which would satisfy the needs of obligate aerobic micro-organisms. Swatek (1963) mentioned that liquid hydrocarbons like jet, rocket and diesel fuel have adequate oxygen absorbent qualities.

DEGME and EGME were ineffective at both low and high temperatures. All the $C$. resinae isolates from the environment and fuel sources (ex feather, soil, jet and diesel fuel from N.Z. and Australia) were tested in EGME and Biobor JF treated systems and appeared to be insensitive. It became evident that all $C$. resinae isolates (whether isolated in $1960^{\prime}$ s or $1970^{\prime} \mathrm{s}$ ) were insensitive to the compounds. Biobor JF was ineffective against $C$. resinae when used intermittently and in the various proportion of $\mathrm{BH}$ and diesel mixtures. 
Hill (1978) reported that it was effective when used intermittently with fuel to water ratios of less than $400: 1$. The possibility that N.Z. C. resinae (ex diesel) used in this study was unusual in being insensitive to DEGME was investigated. But results showed all the Australian and N.Z. jet and diesel fuel isolates were all insensitive to DEGME.

DML-7 and Proxel AS were biostatic against $C$. resinae and $P$. corylophilum but not against $P$. variotii. They prevented the formation of a mycelial mat but a spore mat ${ }^{1}$ was evident. They showed a relatively high degree of inhibition at a very high dose level of $300 \mathrm{ppm}$. When the fungal spores were exposed intermittently to Proxel AS and DML-7, C. resinae was inhibited at $100 \mathrm{ppm}$ in contrast to $300 \mathrm{ppm}$ during the continuous exposure. At above $50 \mathrm{ppm}$, Kathon 886 provided total control of $C$. resinae, Penicillium spp. and P. variotii.

Kathon 886 is fuel and water soluble and maintains an effective biocidal activity at the water/fuel interface. Recently, the RollsRoyce Ltd. in England have given approval to the use of Kathon 886 as an additive to fuel for industrial, marine diesel engines and also gas turbines (Rohm and Haas Ltd. pers. comm.).

In the agar slide culture experiment, benomyl, imazalil and Kathon 886 in the fuel caused a large reduction in the viability of both spores and hyphae of $C$. resinae at concentration of between 10-50 ppm. Germination of spores was not suppressed but germ tube outgrowths was halted within 3 days of emergence. Suppression of germination in benomyl treated fuel was expected as a mode of action of the biocide is by interference with the mitotic process (Davidse, 1973) and the other compounds must have also acted upon the actively metabolizing cells. Abnormalities like leakage of the cell content were apparent in the benomyl treated conidia. Similar observations were made by Van Gestel (1986) when Penicillium spp. spores were treated with azole fungicides. Paecilomyces variotii and Penicillium digitatum were insensitive to imazalil and benomyl respectively.

1 viable concentration of spore inoculum at the fuel/water interface 
But it was possible to control $P$. variotii when imazalil was used in combination with benomyl at a low concentration. Hence the efficiency of imazalil could be increased in the presence of benomyl.

Penicillium digitatum is known to cause citrus green mold and was found to be insensitive to control by benomyl in Australia and Japan (Wataru, 1975; Jones, 1977). It was controlled by imazalil at $20 \mathrm{ppm}$. $\quad P$. digitatum occurred as spores in diesel fuel and was regularly isolated from the atmosphere surrounding the tanks.

The $\mathrm{BH} /$ diesel fuel growth method using medical flat bottles was a reliable method of screening active compounds which gave results in 6 weeks and the same results could be obtained within 8 days using conidial suspension in the agar slide/diesel mixture method. The agar slide method may also be used for dose response studies. In the poison plate test, the effect of increasing fungicide concentration on the growth rate of a known amount of fungal mycelia may be directly observed and measured. The 'natural' two litre fuel test system simulated the actual field condition and provided an opportunity to observe the effectiveness of the biocide in natural conditions.

Studies by Neihof and Bailey (1978) indicated that suitable fuel biocides should have water to fuel partitioning values as high as 1120:1. Rapid partitioning into any water present in the fuel system, would possibly cause biocidal loading to be depleted and provide inadequate microbiological protection by the time the fuel reaches the shipboard tanks. Except for benomy1 and imazalil, all the other products tested were more water soluble than fuel soluble, thereby having the ability to partition rapidly into the aqueous phase with increasing amount of water. The partition coefficient of a compound is also affected by temperature and agitation (Hitzman, 1964; Elphick and Hunter, 1968). For example the coefficient of EGME in water to fuel at $18^{\circ} \mathrm{C}$ is $800: 1$ whereas at $27^{\circ} \mathrm{C}$ it is $200: 1$. Thus, at lower temperatures, EGME becomes more concentrated in the water phase and hence its anti-icing properties are enhanced. However, the increase in biocidal activity with increased temperature compensates for this change in partition and the net result is an increase in antifungal activity as temperature rises (Thomas and Hill, 1977). 
However, when one considers the possible implication of these factors which may intervene in practical application of biocide to frigates, the situation becomes more complex. Agitation may not be a problem in the storage tank but agitation of the fuel and water phases during sailing will increase the rate of partitioning of the water soluble biocide. On the other hand dilution of biocide-treated fuel with untreated fuel at subsequent refuelling points and leaching of biocide from the fuel and dilution of biocide in the water phase by precipitated or condensed water will serve to diminish biocidal efficiency. Of perhaps even greater importance are the environmental factors which will dictate whether, and to what extent, microbial growth will occur in a particular ship. C. resinae and other microorganisms isolated from diesel fuel systems are capable of rapid growth at normal room temperature, provided water and water soluble mineral nutrients are available.

Smith and Crook (1983) found using 14C labelled benomyl a partition ratio of water to fuel of 10:90. As the partition of benomyl into the aqueous phase was low, the depletion of the biocidal properties by loss of water present would occur only slowly. Imazalil may behave similarly to benomyl being more fuel soluble than water soluble and may have a similar partition ratio. Janssen Pharmaceutica Ltd., (the suppliers of imazalil) have obtained a partition coefficient in an octanol-water system of 4:1 at $\mathrm{pH} 9$. The solubility of imazalil decreases at lower $\mathrm{pH}$ and the same effect may be observed in 'dieso'-water system.

The $\mathrm{pH}$ in the $\mathrm{BH} /$ diesel fuel phases was closely monitored, although none of the compounds affected the $\mathrm{pH}$ at the end of 6 weeks. Hill (1982) suggested that biocides particularly those functioning by virtue of a formalin release mechanism have little activity against yeasts and filamentous fungi, and these organisms may present a serious secondary infection hazard after the more vigorous bacteria have been suppressed, as the result of the change in $\mathrm{pH}$ in the last system. To guard against this contingency, a mixture of biocides may be used and also it is essential to achieve an initial high effective concentration to prevent micro-organisms developing resistance to inhibitors by natural selection. 
Therefore Miller, Mohan and Strickland (1975) have suggested that a system in which biocide concentration is at least three times the minimum inhibitory concentration (MIC) would be expected to prevent such development of resistance.

In the field, the compounds tested showed variation in their effectiveness towards the predominant fungi, when compared with the results obtained in the laboratory. Biobor JF and DEGME showed significant reduction of $C$. resinae but not Penicillium spp. and $P$. variotii. Benomyl and imazalil controlled the major fuel contaminating fungi whereas Proxel AS and DML-7 were inhibitory

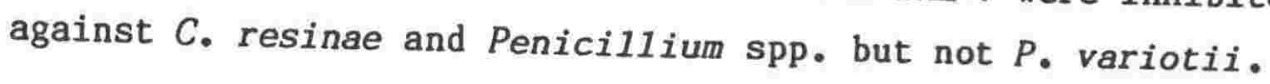

It may be suggested that the crucial test of the effectiveness of a water/fuel soluble compound is a full scale field exposure evaluation and careful analysis under conditions which arise in practice. The field exposure test under conditions of expected use was the primary criterion for evaluating the effectiveness of biocide treatments and was the standard against which the laboratory biocide test method was calibrated. The majority of the published data on biocides have been collected from laboratory studies rather than based on field evaluation. Field trials may prove to be expensive and unpredictable due to the influence of uncontrollable factors. In this work even though the compounds were submitted to the field test after careful scrutiny in the laboratory, some variable results were obtained for DEGME, Biobor JF and imazalil.

Laboratory test data comprised the bulk of the information used in making the decision to proceed with the field test. It must be remembered that the laboratory evaluation of a microbial problem is carried out under conditions that attempt to simulate field conditions. These conditions necessarily differ from the wide spectrum of field conditions in order to meet workable and practical laboratory limitations. It is, therefore, not possible to accurately predict the performance of a biocide in the field on the basis of its laboratory performance. 
DML-7 and Proxel AS have a biostatic effect against $C$. resinae and Penicillium spp. in the laboratory and field. A high proportion of both the compounds migrate to the aqueous phase and may decompose to form toxic compounds, to maintain a low level of contamination in water/diesel interface (DSIR per. comm.). It was obvious in the field study, that there was a large fluctuation in the number of CFU recovered from the treated tanks. The fluctuation in the fungal colonies was more evident in the case of biostats than biocides. For example imazalil and benomyl effectively controlled the predominant fungi and such fluctuation was not observed. The fluctuation in the fungal colonies in the tanks could be due to a number of factors: clumping of fungal mycelium and spores, settling effect, interaction among the dominant fungi, seasonal and biocide effect.

The interaction of all these factors and undoubtedly others, suggest that there may well be a very narrow borderline between a set of conditions under which a fuel tank will remain virtually free of microbiological growth and those under which significant growth will occur. When conditions near to this borderline prevail, intermittent use of even a relatively inefficient compound such as DEGME and Biobor JF may be sufficient to swing the balance in favour of trouble-free service. On the other hand, under really severe operating conditions, an even more efficient compound such as Proxel AS or DML-7 may fail to keep the problem in check as the biocide migrates rapidly into the water phase, therefore it may be necessary to be used very frequently. During situations of heavy microbial contamination, imazalil, benomy 1 and Kathon 886 may be recommended, as these compounds partition gradually into the aqueous phase, maintaining a sterile water bottom and frequent topping would not be necessary.

Micro-organisms can survive in fuel in a dormant state for months or even years without causing any problem, but if the fuel comes in contact with water, the micro-organisms can then proliferate and grow into large masses of mycelial growth which can cause some of the problems previously reported. With continued emphasis placed on good-housekeeping and good fuel-handling practices, it would appear that the microbial problem is subordinate and arises only after continued disregard for established and proven fuel-handling practices. 
As none of the tested compounds showed any deleterious effect on engine performance, nor corrosion at fairly high concentration, probably all the seven compounds may be recommended for further vigorous testing.

Tolerance of micro-organisms to compounds like DEGME and Biobor JF may be suspected but there is no direct evidence. It is becoming increasingly important to have alternate biocides like Proxel AS, DML-7, imazalil, benomyl and Kathon 886 to combat the fuel contaminants. Each of these compounds may be selected based on the severity of the condition.

Benomyl and imazalil have many of the advantages of the ideal fuel biocides. They are agricultural fungicides, easily available, active in low concentration, present no corrosion or residue problems, and their by-products on decomposition may be less toxic than those of DML-7 and Proxel AS. Thus a higher concentration as a disinfectant treatment or lower concentration for continuous dosage on their own or possibly in combination may be used. They may be good candidates as an alternative to the fuel biocide used at present. 
7.

This study established that:

(i) The predominant contaminants of middle distillate fuel ('dieso') are Cladosporium resinae, Paecilomyces variotii and Penicillium corylophilum.

(ii) Penicillium spp. and Paecilomyces variotii are capable of growing in diesel fuel and have the potential to cause major problems in the fuel systems in addition to Cladosporium resinae.

(iii) Interactions exist among the predominant microbial contaminants.

(iv) Biocides effective against $C$. resinae, Paecilomyces variotii and Penicillium spp. are available.

The present investigation showed that the storage tanks surveyed at Devonport, Auckland were contaminated with microorganisms. Cladosporium resinae, Paecilomyces variotii and Penicillium spp. were consistently isolated and were capable of forming dark mycelial mats at the water/diesel interface. Bacteria occurred infrequently and in low numbers.

Methods developed for the detection of fungi and bacteria showed that a filtration method using membrane filters gave higher colony counts and more reliable results. V-8 juice agar and malt extract agar media poured over the filters were found best for isolating $C$. resinae and other micro-organisms.

There are numerous sources of contamination of storage fuel tanks by $C$. resinae and other micro-organisms. Microbial contamination may occur through breather vents which allow air borne and soil borne spores to enter with rainwater or dust particles. Contamination may also occur from the introduction of contaminated fuel into the fuel distribution system especially from the supplier and the tank cleaning 
It is evident, although growth of the major fungi was slow, that these fungi may obtain sufficient nutrients from diesel to grow in the presence of water. It is possible that once growth has been established and the organisms have become attached to the walls of the fuel tanks, fragments would be easily dislodged and spores produced would recontaminate fuel indefinitely.

As the fuel tanks were shown to contain more than one fungal contaminant, interaction among the different species of microorganisms become inevitable. A laboratory investigation showed that C. resinae may grow profusely, suppressing the other organisms in the Bushnell-Haas mineral salt medium/diesel fuel phases containing the high and balanced nutrient but not in seawater/diesel fuel, tapwater/diesel and distilled water/diesel phases. But Penicillium corylophilum survived prolonged immersion ( 6 weeks) in seawater/diesel and completely suppressed $C$. resinae and $P$. variotii in a mixed inoculum situation. $C$. resinae grew on the same seawater/diesel phases after the growth and removal of $P$. corylophilum but these two species did not grow together.

The ultrastructure of Cladosporium spp. spores showed a thick cell wall of several successive layers. The plasma-membrane, underlying the cell wall was resolved to be a trilamellar structure. Spores contained a single nucleus and many unidentified membrane bound bodies and membrane systems. Light and dark electron-dense bodies inside and outside the vacuoles were common to the species. Microbodies were evident in all the species and no difference in the intracellular structures were observed among the Cladosporium spp.

Attempts were made to control microbiological growth in N.Z. navy storage tanks at Devonport using biocides where physical methods of control have become impossible. The effects of commercially available (EGME, DEGME, Biobor JF) and experimental biocides (DML-7, Proxel AS, Kathon 886, benomy1 and imazalil) on microbiological growth in diesel fuel were studied. All the available products were screened

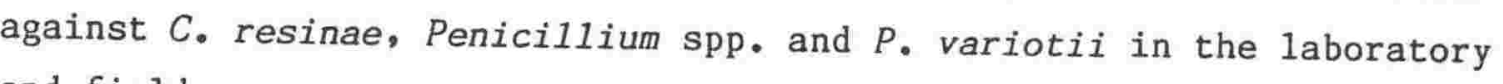
and field. 
EGME, DEGME and Biobor JF were neither biostatic nor biocidal to the fungi in the laboratory but in the field they suppressed $C$. resinae but had no effect on the other micro-organisms present. DML-7 and Proxel AS were inhibitory while benomy1, imazalil and Kathon 886 gave total control of the predominant micro-organisms especially $C$. resinae. All the compounds tested passed the engine performance and corrosion tests. 
8.

(i) Regular surveillance and monitoring of the stored fuel for the predominant contaminants is a necessity because this study demonstrated the presence of $C$. resinae, Penicillium spp. and Paecilomyces variotii in diesel fuel.

(ii) 'Good-housekeeping' procedures should be implemented which eliminate free water from the tanks thereby minimizing the likelihood of microbial growth becoming established. The pumping of water from the separating tank into tank 3 should be completely stopped and the fuel passed through filter systems before pumping into storage tanks.

(iii) The concrete underground tanks may no longer be suitable for storing clean fuel and a complete re-design of the fuel system and storage tanks at Devonport should be considered. Upright steel tanks with conical bases would allow water to settle at the bottom from which it could be drained easily.

(iv) Personnel who are handling and managing the fuel should be made aware of the consequences of microbiological contamination of fuel.

(v) If the fuel is kept free from contamination in the storage tanks, there should be little trouble in the shipboard tanks unless these are water-compensated tanks. Intake of tapwater rather than coastal or harbour water would help to keep the problem in check.

(vi) There is an urgent need to treat all fuel entering/TCV with a biocide before it is pumped back into the shipboard tanks. 
(vii) If it is impossible to control microbiological contamination by maintaining a water-free system, the effectiveness of surface active biocides for inhibiting microbial growth and reducing sludge generation in storage tanks and water compensated tanks in ships ought to be considered. The water-soluble inhibitors like DML-7 and Proxel AS may migrate rapidly into the aqueous phase and it might be necessary to replenish the fuel system regularly with the compounds. These inhibitors should be used only in clean tank systems. Biocides such as benomy1, imazalil and Kathon 886, which are active at low concentrations may be used to dose the tanks intermittently or even injected along the fuel line, whenever there is a fresh supply of fuel introduced into the tanks.

(viii) If biocides are used, it is important to monitor the fuel regularly in order to maintain an effective concentration at the fuel/water interface.

(ix) The use of DML-7, Proxel AS, benomyl, imazalil and Kathon 886 in fuel would require clearance by engine manufacturers and other fuel authorities before using in the shipboard tanks.

(x) As a precaution, since most of the compounds are capable of producing toxic by-products in the aqueous phase, it may be advisable to neutralise the compounds before draining the water into the wharf or sea. The chemical manufacturers must provide suitable chemical antagonists that will render the biocides safe for the environment. 


\section{BIBLIOGRAPHY}

Albert, A. 1979. Selective toxicity: The physiochemical basis of therapy. 5th Edition. Halsted Press, A Division of John Wiley and Sons, Inc., New York.

Al Haidary, N.K. 1977. Microbial spoilage of hydraulic and bearing oils. $\mathrm{Ph}_{0} \underline{\mathrm{D}}$. Thesis, University College, Cardiff.

Allen, F.H. 1945. The microbiological aspects of gasoline inhibitors. Journal of the Institute of Petroleum 31: 9-15.

Anon. 1981. Fungus stops buses. Evening Post, Wellington, New Zealand. 3rd April.

Anon. 1986 0il-eating bus-stopper bug curbed. Evening Post, Wellington, New Zealand. 10th February.

Arnold, J.B. 1983. Fuels, filters, and fungi. Journal of Naval Engineering 28(1): 56-63.

Avers, C.J. 1971. Peroxisomes of yeast and other fungi. Sub-cellular Biochemistry 1: 25-37.

Bakanauskas, S. 1958. Bacterial activity in JP-4 fuel. Wright Air Development Center Technical Report 58-32. March.

Beerstecher, E. 1954. Petroleum Microbiology. 1st Edition. Elsevier Press, Houston. pp. 375.

Bennett, E.0. 1974. The deterioration of metal cutting fluids. Progress in Industrial Microbiology 13: 121-150.

Berner, N.H., and Ahearn, D.G. 1977. Observations on the growth and survival of Cladosporium resinae in jet fuel. Developments in Industrial Microbiology 18: 705-710. 
Berry, H., and Michaels, I. 1950. The evaluation of the bacterial activity of ethylene glycol and some of its monoalkyl ethers against Bacterium coli. Journal of Pharmacy and Pharmacology 2: 27.

Bracker, C.E. 1967. Ultrastructure of fungi. Annual Review of Phytopathology 5: 343-374.

Brenner, D.M., and Carrol1, G.C. 1968. Fine-structural correlates of growth in hyphae of Ascodesmis sphaerospora. Journal of Bacteriology 95: 658-671.

Bruce, N. 1982. A laboratory study of microbial growth in simulated fuel tanks. International Biodeterioratiion Bulletin 18(3): $87-91$.

Brushaber, J.A., and Jenkins, S.F. Jr. 1971. Lomasomes and vesicles in Poria monticola. Canadian Journal of Botany 49: 2075-2079.

Bushnel1, L.D., and Haas, H.F. 1941. The utilization of certain hydrocarbons by micro-organisms. Journal of Bacteriology 41: 653-673.

Chiba, M., and Doornbos, F. 1974. Instability of benomyl in various conditions. Bulletin of Environmental Contamination and Toxicology 11: 273-274.

Christensen, C.M., Kaufert, F.H., Schmitz, H., and Allison, J.L. 1942. Hormodendrum resinae (Lindau), an inhabitant of wood impregnated with creosote and coal tar. American Journal of Botany 29: 552-558.

Clemons, G.P., and Sisler, H.D. 1969. Formation of a fungitoxic derivative from Benlate. Phytopathology 59: 705-706

Cole, G.T. 1973. U1trastructure of conidiogenesis in Drechslera sorokiniana. Canadian Journal of Botany 51: 629-638. 
Cole, G.T. 1974. Conidiophore and Conidium ontogeny in Spegazzinia tessarthra. Canadian Journal of Botany 52: 1259-1264.

Cole, G.T. 1975. A preparatory technique for examination of imperfect fungi by scanning electron microscopy. Cytobios 12: 115-121.

Cole, G.T. 1976. Conidiogenesis in pathogenic hyphomycetes. I. Sporothrix, Exophiala, Geotrichum and Microsporum. Sabouraudia 14: 81-98.

Cole, G.T., and Samson, R.A. 1979. Patterns of development in conidial fungi. Pitman. London.

Collins, C.H., and Lyne, P.M. 1976. Microbiological methods. 4th Edition. Butterworths Company Publishers.

Cooney, J.J., and Kula, T.J. 1970. Growth and survival of organisms from hydrocarbon fuel systems. International Biodeterioration Bulletin 6(3): 109-114.

Cooney, J.J., and Proby, C.M. 1971. Fatty acid composition of Cladosporium resinae grown on glucose and hydrocarbons. Journal of Bacteriology 108: 777-781.

Cooney, J.J., Siporin, C., and Smucker, R.A. 1980. Physiological and cytological responses to hydrocarbons by the hydrocarbon-using fungus Cladosporium resinae. Botanica Marina 23: 227-232.

Davidse, L.C. 1973. Antimitotic activity of methyl benzimidazol-2-y1 carbamate (MBC) in Aspergillus nidulans. Pesticide Biochemistry and Physiology 3: 317-325.

De Gray, R.J., and Killian, L.N. 1960. Bacterial slime and corrosion in petroleum product storage. Industrial and Engineering

Chemistry 52(12): 58A-60A. 
De Gray, R.J., and Fitzgibbons, W.0. 1966. Mechanism of microbiocida1 action of organo-borates in fuel-water systems. Developments in Industrial Microbiology 7: 384-391.

de Male, M.F.L., Salvarezza, R.C., and Videla, H.A. 1979. Microbial contaminants influencing the electrochemical behaviour of aluminium and its alloys in fuel-water systems. International Biodeterioration Bulletin 15(2): 39-44.

de Meybaum, B.R., and de Schiapparelli, E.R. 1980. Corrosion by microorganisms of jet aircraft integral fuel tanks. International Biodeterioration Bu1letin 16(2): 31-36.

de Schiapparelli, E.R., and de Meybaum, B.R. 1980. Role of dodecanoic acid in microbiological corrosion of jet aircraft integral fuel tanks. International Biodeterioration Bulletin 16(3): 61-66.

de Vries, G.A. 1952. Contribution to the knowledge of the genus Cladosporium Link ex Fr. Bibliotheca Mycologia 3: 46-56.

Edmonds, P. 1966. Microbiological investigations. Technical Documentary Report No. RTD - TDR $-63-\underline{4118}$, Part III. January. Airforce Aero Propulsion Laboratory Research and Technology Division, Airforce Systems Command Wright-Patterson Airforce Base, Ohio.

Elphick, J.J., and Hunter, S.K.P. 1968. Evaluating biocidal fuel additives for intermittent use in aircraft fuel systems. In: Biodeterioration of Materials. A.H. Walters and J.J. Elphick, editors. Elsevier. 364-370.

Fass, R., and Miller, G. 1980. Microbial growth in storage of jet fuel and diesel fuel. Summary of Report for May 1979 - April 1980, Israel Institute for Biological Research October.

Fawcett, D.W. 1966. An atlas of fine structure: The Cell, Its Organelles and Inclusions. Saunders, Philadelphia. 448pp. 
Foster, J.W. 1962. Hydrocarbons as substrates for micro-organisms. Antonie Van Leewenhoek 28: 241-274.

Frederick, S.E., Gruber, P.J., and Newcomb, E.H. 1975. Plant Microbodies. Protoplasma 84: 1-29.

Ganser, P. 1940. Pipeline corrosion caused by anaerobic bacteria. Gas Journal 231: 371-373.

Genner, C., and Hill, E.C. 1981. Fuels and oils. In: Microbial Biodeterioration. A.H. Rose, editor. Academic Press, London. 260-301.

Green, R.H., Olson, R.L., Gustan, E.A., and Pilgrim, A.J. 1967. Microbial survival in propellants before and after rocket firings. Developments in Industrial Microbiology 8: 227-233.

Hammerschlag, R.S., and Sisler, H.D. 1973. Benomyl and methyl-2benzimidazole carbamate (MBC). Biochemical, cytological and chemical aspects of toxicity to Ustilago maydis and Saccharomyces cerevesiae. Pesticide Biochemistry and Physiology 3: 42-54.

Hansen, D.J., Tighe-Ford D.J., and George, G.C. 1981. Role of the mycelium in the corrosive activity of Cladosporium resinae in a dieso/water system. International Biodeterioration Bulletin 17(4): 103-112.

Hawker, L.E., and Linton, A.H. 1979. Micro-organisms function, form and environment. 2nd Edition. Edward Arnold Publishers, London.

Hayat, M.A. 1970. Principles and techniques of electron microscopy. Biological applications. Littleton Educational Publishing.

Hayat, M.A. 1981. Fixation for electron microscopy. Academic Press, London.

Hazzard, G.F. 1961. Fungal growth in aviation fuel systems. Part I. Defence Standards Laboratories (Australia). Report 252, 33pp. 
Hazzard, G.F. 1963. Fungal growths in aviation fuel systems. Part 4. Fungi in aviation fuel systems in Australia and the Far East. Defence Standards Laboratory (Australia). Report 252, 52pp.

Heath, I.B. 1977. Morphology and ontogeny of microbodies in the oomycete fungus Sapromyces elongatus. Canadian Journal of Botany 55: 556-562.

Hedrick, H.G., Miller, C.E., Halkias, J.E. Hildebrand J.F., and Gilmartin J.N. 1965. A current evaluation of microbiological corrosion system for studying the effects on structural aluminium alloys. Developments in Industrial Microbiology 6: 117-123.

Hedrick, H.G., and Carroll, M.T. 1966. Laboratory investigation of micro-organism effects related to aircraft fuel systems. Developments in Industrial Microbiology 7: 372-383.

Hendey, N.I. 1964. Some observations on Cladosporium resinae as a fuel contaminant and its possible role in the corrosion of aluminium alloy fuel tank. Transaction British Mycological Society $47(4): 467-475$.

Hendey, N.I., Bagdon, V.U., Ernst, G.E., Klemme, D.E., and Leonard, J.M. 1971. Biocidal properties of an anti-icing additives. Naval Research Laboratory Report No. NRL-7337.

Hill, E.C., Evans, D.A., and Davies, I. 1967. The growth and survival of micro-organisms in aviation kerosene. Journal of the Institute of Petroleum 53: 280-284.

Hill, E.C., Davies, I., Pritchard, J.A.V., and Byron, D. 1967. The estimation of micro-organisms in petroleum products. Journal of the Institute of Petroleum 53(524): 275-279.

Hill, E.C., and Hughes, D.G. 1969. Microbiology in heavy industry. In: Proceedings of Global Impacts of Applied Microbiology, III, pp 196-202. Bombay . 
Hill, E.C. 1970. A simple rapid microbiological test for aircraft fuel. Aircraft Engineering. July 24-26.

Hi11, E.C. 1975. Biodeterioration of petroleum products. In: Microbial Aspects of the deterioration of materials. D.W. Lovelock and R.J. Gilbert, editors London, Academic Press. 127-136.

Hil1, E.C. 1978. Biodegradation of hydrocarbon-based products in industrial use. In: Developments in Biodegradation of hydrocarbons, Vol. 1. R.J. Watkinson, editor. 201-277.

Hil1, E.C. 1982. Preservation in specialised areas. B. Fuels and Lubricants. In: Principles and Practice of Disinfection, Preservation and Sterilization. A.D. Russell, W.B. Hugo and G.A.J. Ayliffe, editors. Blackwell Scientific Publication, London. 352-357.

Hitzman, D.O., Shotton, J.0., and Alquist, H.E. 1963. Biocidal effects of PF A 55MB. Paper presented at Society of Automotive Engineers, Aeronautical meeting, Washington D.C. (SAE preprint 683D).

Hitzman, D.O. 1964. The control of bacterial and fungal growth in jet fuels by use of a fuel additive. Developments in Industrial Microbiology 6: 105-116.

Hostetler, H.F., and Powers, E.J. 1963. Bugs, surfactants and woes. Paper presented at the 28th mid year meeting of the American Petroleum Institute's Division of Refining, Philadelphia, May 13.

Houghton, D.R., and Gage, S.A. 1979. Biology in ships. Transactions of the Institute of Marine Engineers 91: 1-7.

Isenberg, D.L., and Bennett, E.0. 1959. Bacterial deterioration of emulsion oils. II. Nature of the relationship between aerobes and sulphate-reducing bacteria. Applied Microbiology 7: 121-125. 
Jones, L.C. 1977. Australian records of resistance of fungi to fungicides. Australian Plant Pathology Society Newsletter 6(1): 13-15.

Karnovsky, M.J. 1965. A formaldehyde-glutaraldehyde fixative of high osmolality for use in electron microscopy. Journal of Cell Biology 27: 137A-138A.

Kelley, B.E. 1967. 'USS IWD JIMA Aviation gasoline tanks, elimination and control of contamination'. NAVSECPHILADIV Report of Travel 10330 (FA-157), 27 January.

Kelley, R.O., Decker, A.F., and Bluemink, J.G. 1973. Ligand-mediated osmium binding: its application in coating biological specimens for scanning electron microscopy. Journal of U1trastructure Research 45: 254-258.

King, R.A., and McKenzie, P. 1977. Microbial degradation of marine lubricating oil. Transactions of the Institute of Marine Engineers 89: 37-43.

Klausmeier, R.E., and Andrews, C.C. 1981. Plastics. In: Microbial Biodeterioration. A.H. Rose, editor. Academic Press, London. 431-472.

Klemme, D.E., and Leonard, J.M. 1960. Microbial inhibitors for systems of jet fuel and water. Naval Research Laboratory Report No. 5501 AD-244-511.

Klemme, D.E. 1968. Sulfate-reducing bacteria in fuel tanks of aircraft carriers. Naval Research Laboratory Memorandum Report 1943, November.

Klemme, D.E., and Neihof, R.A. 1976. An evaluation in large-scale test systems of biocides for control of sulfate-reducing bacteria in shipboard fuel tanks. Naval Research Laboratory Memorandum Report 3212 . 
Kostenbauder, H.B. 1977. Disinfection, sterilization and preservation. 2nd edition. S.S. Block, editor. Lea and Febiger, Philadelphia, P.0., 912-932.

Kramer, C.L., Eversmeyer, M.G., and Collins, T.I. 1976. A new 7-day spore sampler. Phytopathology 66: 60-61.

Landsdown, A.R. 1965. Microbiological attack in aircraft fuel system. Journal of Royal Aeronautical Society 69 (659): 763-767.

London, S.A., Finefrock, V.H., and Killian, L.N. 1964. Microbial activity in airforce jet fuel systems. Developments in Industrial Microbiology 6: 61-91.

Lonsane, B.K., Singh, H.D., and Baruah, J.N. 1975. Codification of Petroleum Hydrocarbon Utilizing Micro-organisms. Journal of Scientific and Industrial Research 11: 614-624.

Liggett, S. 1976. The extent and significance of micro-organisms in hydrocarbon fuel oil storage systems. M.Sc. Thesis, University of Wales, Cardiff.

Lukins, H.B. 1962. On the utilization of hydrocarbons methyl ketones and hydrocarbon by mycobacteria. Thesis, University of Texas. Dissertation Abstracts 23(1): 29-31.

Marsden, D.H. 1954. Studies on the creosote fungus, Hormodendrum resinae. Mycologia 46: 161-183.

May, M.E., and Neihof, R.A. 1981. Growth of Cladosporium resinae in seawater/fuel systems. Developments in Industrial Microbiology 22: 781-787.

McKeen, W.E. 1971. Woronin bodies in Erysiphe graminis. Canadian Journal of Microbiology 17: 1557-1560. 
McKenzie, P., Akbar, A.S., and Miller J.D. 1976. Fungal Corrosion of Aircraft Fuel Tank Alloys. In: Microbial Corrosion affecting the petroleum industry. Institute of Petroleum Microbiology Group Symposium. IP 77-001, 37-50.

Millar, A.H., Mohan, R.R., and Strickland, B.R. 1975. Studies on hydrocarbon soluble biocides of differing partition coefficients. Institute of Petroleum IP76-007, 1-10.

Miyoshi, M. 1895. Die durch bohung von membranen durch philzfaden. Jahrbuch Fuer Wissenschaftliche Botanik 28: 269-289.

Moore, R.T. 1965. The ultrastructure of fungal cells. The Fungi, I. G.C. Ainsworth and A.S. Sussman, editors. An advanced treatise, Academic Press, New York. 95-118.

Nicot, J., and Zakartchenko, V. 1966. Remarques sur la morphologie et la biologie du Cladosporium resinae (Lindau) de Vries. Revue de Mycologie (Paris) 31: 48-74.

Nilsson, T., and Henningsson, B. 1977. On the occurrence of Phialophora species in preservative treated wood in ground contact. 9th Annual meeting of the International Research Group Wood Preservers. Noordwijk Aan Zee.

Neihof, R.A., and Bailey, C.A. 1978. Biocidal Properties of Anti-Icing additives for Aircraft Fuels. Applied and Environmental Microbiology 35(4): 698-703.

Neihof, R.A., Bailey, C.A., Patouillet, C., and Hannan, P.J. 1979. Photodegradation of Mercaptopyridine $-\mathrm{N}$ - Oxide Biocides. Archives of Environmental Contamination and Toxicology 8: 355-368.

Neihof, R.A. 1980. Microbial Contamination of ship fuels. 28th Conference on Microbiological Deterioration of Military Material, Natick Laboratories. 
Neihof, R.A., and May, M.E. 1982. Survey of contamination in fuel tanks of DD-963 Class ships. Naval Research Laboratory Memorandum Report No. 4853, July: 1-49.

Neihof, R.A., and May, M.E. 1983. Microbial and particulate contamination in fuel tanks on naval ships. International Biodeterioration Bulletin 19(2): 59-68.

Parbery, D.G. 1968. The role of Cladosporium resinae in the corrosion of aluminium alloys. International Biodeterioration Bulletin 4(2): $78-81$.

Parbery, D.G. 1969. Amorphotheca resinae Gen. Nov. sp. Nov.: the perfect state of Cladosporium resinae. Australian Journal of Botany 17: 331-357.

Parbery, D.G. 1970. The kerosene fungus; Amorphotheca resinae; its biology, taxonomy and control. Ph.D thesis, University of Melbourne, Australia.

Parbery, D.G. 1971. Biological problems in jet aviation fuel and the biology of Amorphotheca resinae. Material und Organismen 6 Bd. Heft 3 : 161-207.

Parbery, D.G., and Thistlewaite, P.J. 1973. Improved mineral salts solution for the growth of Amorphotheca resinae. International Biodeterioration Bulletin 9(1-2): 11-16.

Park, P.B. 1973. Evaluation of biocides for use in aviation fuel. International Biodeterioration Bulletin 9(3): 79-81.

Park, P.B. 1975. Biodetioration in aircraft fuel systems. In: Microbial Aspects of the Deterioration of Materials. D.W. Lovelock and R.J. Gilbert, editors. Academic Press, London. 106-124.

Pitt, J.I. 1979. The genus Penicillium and its teleomorphic states Eupenicillium and Talaromyces. London, Academic Press. 
Rabotnova, I.L., Paushkin, Ya. M., Vishnyakova, T.P., Grechushkina, N.N., Avakyan, Z.A., Vlasova, I.D., and Rrylov, I.F. 1970. Inhibition of the growth of micro-organisms on hydrocarbon media by Quaternary Ammonium salts. Mikrobiologiya 30(4): 642-644.

Reichart, L.M. 1983. Contamination of distillate fuels. MER, July.

Reynold, E.S. 1963. The use of lead citrate at high pH as an electronopaque stain in electron microscopy. Journal of Cell Biology 17: 208-212.

Rivers, C. 1973. The growth, metabolism and corrosive effects of Cladosporium resinae. Ph.D. thesis, University of Manchester.

Rogers, M.R., and Kaplan, A.M. 1968. Screening of prospective biocides for hydrocarbon fuels. Developments in Industrial Microbiology 9: 448-477.

Russ, J.C., and Kabaya, A. 1970. Preparation of samples for scanning electron microscopy. Proceedings 28th Annual EMSA meeting. Claitor's publishing company. New Orleans, La., 380pp.

Russe11, A.D. 1982. Factors influencing the efficacy of antimicrobial agents. In: Principles and Practice of Disinfection, Preservation and Sterilization. A.D. Russe11, W.B. Hugo and G.A.J. Ayliffe, editors. Blackwell Scientific Publicatioins. London. 107-133.

Saunders, J.C., Wotring, W.T., and Taylor, J.F. 1966. Biobor JF in turbine fuel. U.S. Borax Report 20 October.

Sharpley, J.M. 1966. Elementary Petroleum Microbiology. Gulf publishing company, Houston. Texas.

Sheridan, J.E., and Knox, M.D.E. 1970. Note on the discovery of the 'kerosene fungus' Amorphotheca resinae Parbery (con. stat. Cladosporium resinae (Lindau) De Vries) in New Zealand. New Zealand Journal of Science 13: 140-142. 
Sheridan, J.E., Nelson, J., and Tan, Y.L. 1971. Studies on the 'kerosene fungus' Cladosporium resinae (Lindau) De Vries. Part I. Tuatara 19: 21-42.

Sheridan, J.E., and Nelson, J. 1971. The selective isolation of the

'kerosene fungus' Cladosporium resinae from the air. International Biodeterioration Bulletin 7(4): 161-162.

Sheridan, J.E., Stee1, J., and Knox, M.D.E. 1971. The natura1 occurrence of the 'kerosene fungus' Amorphotheca resinae in New Zealand soils. New Zealand Journal of Science 14(1): 147-160.

Sheridan, J.E. 1972. Studies on the kerosene fungus Amorphotheca resinae in New Zealand. International Biodeterioration Bulletin $\underline{8(2)}: 65-74$.

Sheridan, J.E., Tan, Y.L., and Nelson, J. 1972. Studies on the 'kerosene fungus' Cladosporium resinae (Lindau) De Vries. Part III. Morphology, Taxonomy and Physiology. Tuatara 19(3): 130-165.

Sheridan, J.E., and Troughton, J.H. 1973. Conidiophores and conidia of the 'kerosene fungus' Cladosporium resinae in the light and scanning electron microscopes. New Zealand Journal of Botany 11: 145-152.

Sheridan, J.E., and Soteros, J.J. 1974. A survey of fungi in jet aircraft fuel systems in New Zealand. International Biodeterioration Bulletin 10(4): 105-107.

Sheridan, J.E. 1974. Monitoring for the 'kerosene fungus' Amorphotheca resinae. Revista de Microbiologia (S. Paulo) 5(3): 67-71.

Singer, M. 1976. Laboratory Procedures for assessing the potential of Antimicrobial agents as industrial biocides. Process Biochemistry 11(6): 30-35. 
Siporin, C., and Cooney, J.J. 1975. Extracellular lipids of Cladosporium (Amorphotheca) resinae grown on glucose or on n-alkanes. Applied Microbiology 39(5): 604-609.

Smith, R.N., and Crook, B. 1980. The germination and growth of Cladosporium resinae in fuel oil. Proceedings 4th International Biodeterioration Symposium, Berlin. T.A. Oxley and G. Becker, editors. Pitman, London. 29-36.

Smith, R.N., and Crook, B. 1983. The growth and mortality of Cladosporium resinae in biocide treated fuel oil. Proceedings 5th International Biodeterioration Symposium, Aberdeen. T.A. Oxley and S. Barry, editors. John Wiley and Sons Ltd., New York. 486-493.

Smucker, R.A., and Cooney, J.J. 1981. Cytological responses of Cladosporium resinae when shifted from glucose to hydrocarbon medium. Canadian Journal of Microbiology 27(11), 1209-1218.

Smucker, R.A., and Cooney, J.J. 1983. Cytological and physiological responses of Cladosporium resinae exposed to hydrocarbon. In: Biodeterioration 5. T.A. Oxley and S. Barry, editors. John Wiley and Sons Ltd. London. 361-372.

Sohngen, N.L. 1913. Benzin, petroleum, paraffino und paraffin als kohlenstoff-und energiequelle fur mikroben. Bakteriol Parasitek Infek II 37: 595-609.

Soteros, J.J. 1973. Microbial contaminants of jet fuel in New Zealand. M.Sc. thesis, Victoria University of Wellington, New Zealand.

Spurr, A.R. 1969. A low viscosity epoxy resin embedding medium for electron microscopy. Journal of Ultrastructure Research 26: 31-43.

Stormont, D.H. 1961. "Do Jet Fuel Bacteria Cause Slime, Corrosion?" The Oil and Gas Journal, July 3: 82-84. 
Stormont, D.H. 1962. Boron additive help NYC combat bacterial fouling of diesel fuel. The 0il and Gas Journal, September 17: 133-134

Swatek, F.E. 1963. Fundamentals of microbiological contamination of liquid hydrocarbon fuels. Society of Automotive Engineering Congress, Detroit, Michigan 651A.

Tan, Y.L. 1972. Studies on the morphology, physiology and taxonomy of the 'kerosene fungus' Amorphotheca resinae Parbery (conidial state Cladosporium resinae (Lindau) de Vries. M.Sc. Thesis, Victoria University of Wellington. New Zealand.

Tausson, W.0., and Aleshina, W.A. 1932. Uber die bakterielle sulfatreduktion bei Anwesenheit der Kohlenwasserstoffe. Mikrobiologiya 1: 229-261 (In Russian).

Tausson, T.A. 1939. Oxidation of paraffin by yeast and yeast-like organisms. Mikrobiologiya 8 : 828-833.

Teh, J.S., and Lee, K.H. 1973. Utilization of n-alkanes by Cladosporium resinae. Applied Microbiology 25: 454-457.

Thaysen, A.C. 1939. On the gas evolution in petrol storage tanks caused by the activity of micro-organisms. Journal of the Institute of Petroleum 25(189): 411-415.

Thomas, A.R., and Hill, E.C. 1977. Aspergillus fumigatus and supersonic aviation, 3. Survival. International Biodeterioration Bulletin 13(1): 1-4.

Turner, A.P.F. 1981. Conditions affecting the growth of Cladosporium resinae in ship fuel systems. Bulletin of British Mycological Society 15 (supplement): 6-7.

Turner, A.P.F., Eaton, R.A., and Jones, E.B.G. 1983. Nutritional aspects of ship fuel system contamination by Cladosporium resinae. In: Biodeterioration 5. T.A. Oxley and S. Barry, editors. John Wiley and Sons, New York. 507-516. 
Upsher, F.J. 1976. Thermal inactivation of fungal spores. Institute of Petroleum, London. Paper number IP 76-001.

Van Geste1, J. 1986. Conidiation in some hyphomycetous fungi and the effects thereon of imidazole and triazole fungicides. Ph.D. thesis. Katholieke Universiteit Leuven Faculteit Der Wetenschappen, Belgium.

Vigil, E.L. 1973. Structure and function of plant microbodies. Subcellular Biochemistry 2: 237-285.

Von Arx, J.A., and de Vries, G.A. 1973. Hormoconis - Hyphomycetes sp. type Hormodendrum resinae Lindau. Verhandelingen, Koninklijke Nederlandse Akademie Van Wetenschappen 2(4): 62

Walker, J.D., and Cooney, J.J. 1973. Oxidation of n-alkanes by Cladosporium resinae. Canadian Journal of Microbiology 19(10): 1325-1330.

Walters, A.H. 1971. Microbiological and allied aspects of biodeterioration. Progress in Industrial Microbiology 10: 179-218.

Wataru, I. 1975. On the tolerance of plant pathogenic fungi and bacteria to fungicides in Japan. Japan Pesticide Information Number $23: 13-16$

Wergin, W.P. 1973. Development of woronin bodies from microbodies in Fusarium oxysporum f. sp. Iycopersici. Protoplasma 76: 249-260.

Wood, R.L., and Luft, J.H. 1965. The influence of buffer systems on fixation with osmium tetroxide. Journal of U1trastructure Research 12: 22-45.

Wycislik, E.T., and Allsopp, D. 1983. Heat control of microbial colonization of shipboard fuel system. In Biodeterioration 5 . T.A. Oxley, and S. Barry, editors. John Wiley and Sons, New York. 453-461. 
Zittle, C.A. 1951. Reactions of borate with substances of biological interest. Advances in Enzymology 12: 493-527.

ZoBel1, C.E. 1946. Action of micro-organisms on hydrocarbons. Bacteriological Reviews 10: 1-49.

ZoBe11, C.E. 1950. Assimilation of hydrocarbons by micro-organisms. Advances in Enzymology 10: 443-486. 
APPENDICES 
* Usually the bottom samples are analysed for water contents. The samples were not analysed for water regularly but tank 3 bottom usually contained excessive amount of water.

0 No colonies were observed in that sample.

$+\quad$ Numbers refer to the numbers of fungal colony forming units per litre of fuel calculated from counts of the fuel filtered aliquots of each sample.

T $\quad 0.6 \mathrm{~m}$ from the top of the tank ceiling.

M $2 \mathrm{~m}$ below the surface of the fuel.

B $6 \mathrm{~m}$ below the surface of the fuel.

V.B. Very bottom, approximately $10 \mathrm{~mm}$ from the VB to $300 \mathrm{~mm}$. 


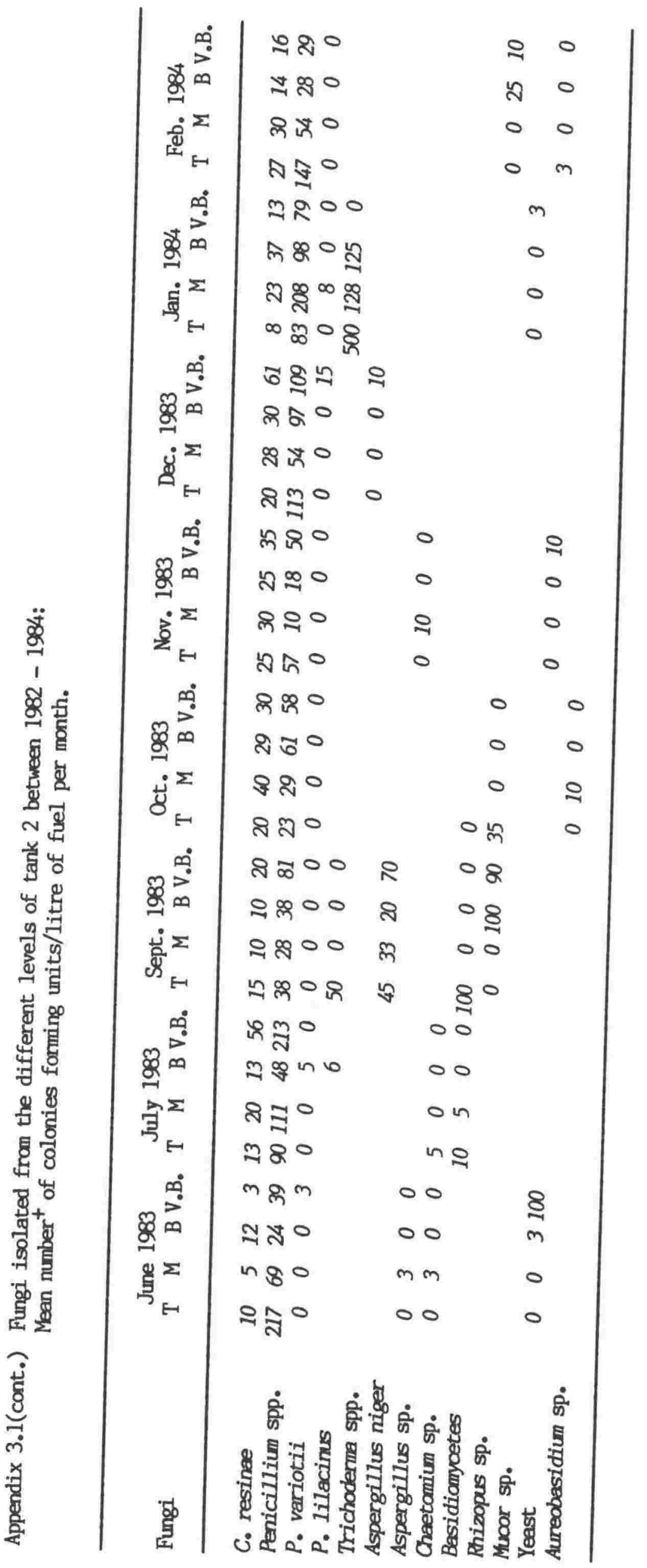




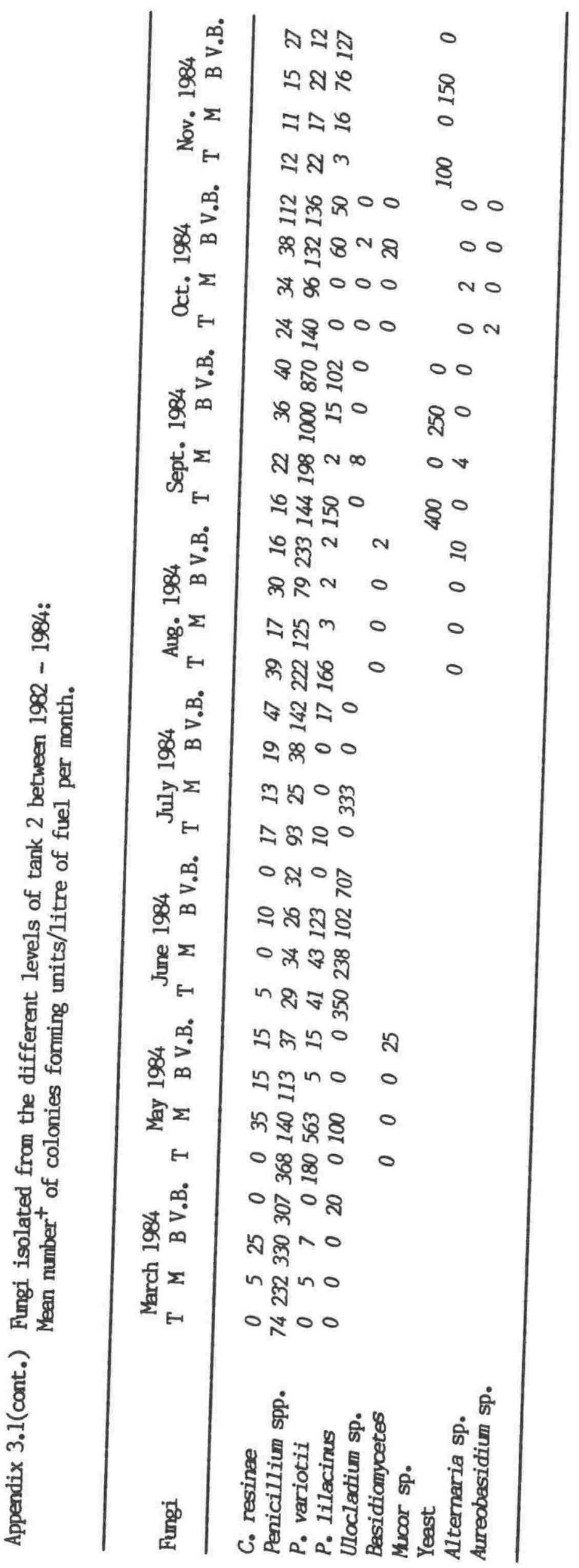




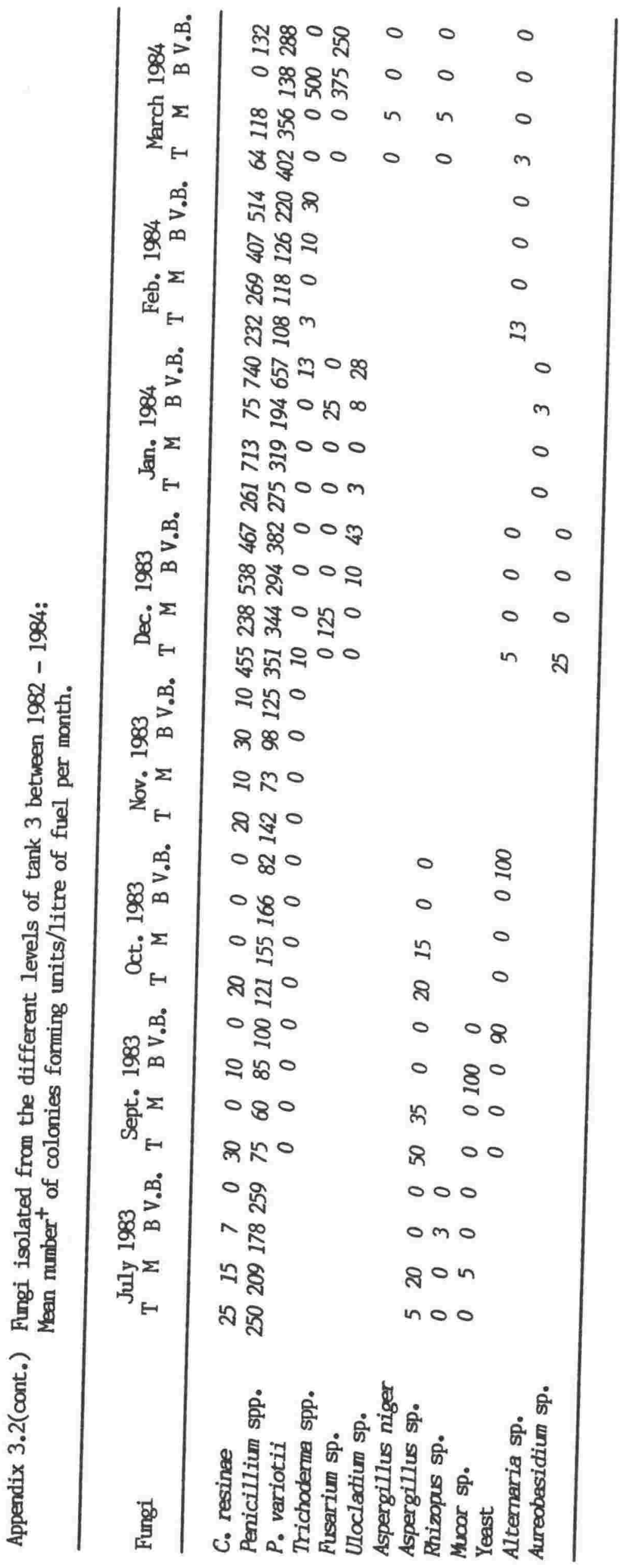




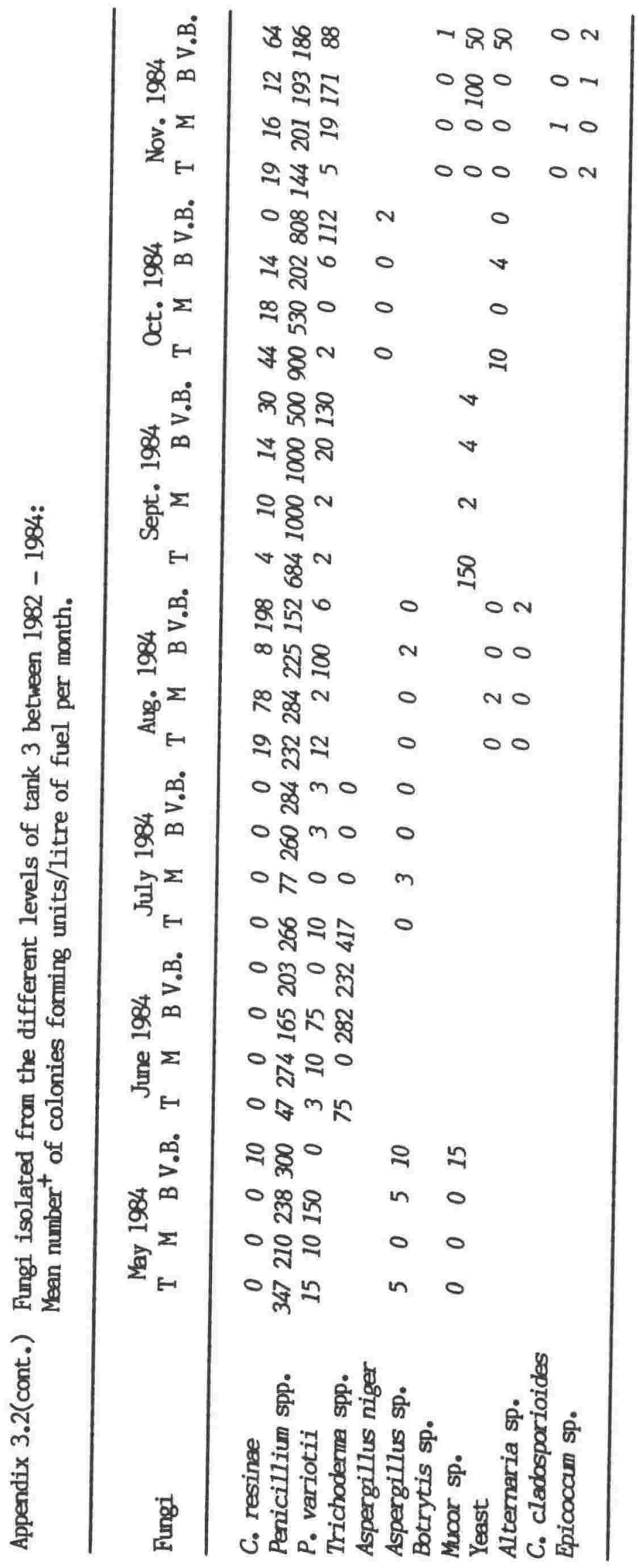




\section{Appendix 5.1 Partition coefficients of imazalil in the octanol-water system. \\ Results from the physico-chemical department (Dr J. Peiters, Janssen Pharmaceutica N.V., Belgium).}

$\begin{array}{cr}\text { Buffer System } & \text { R23979 (A 09/1) } \\ \text { pH } & \log R\end{array}$

$\begin{array}{lll}\text { Citrate-phosphate } & 2.15 & 0.42 \\ & 4.05 & 1.43 \\ & 6.18 & 3.27 \\ & 8.02 & 3.90\end{array}$

$\begin{array}{lll}\text { Borate- } \mathrm{NaOH} \quad 9.82 & 3.87\end{array}$

$$
\begin{aligned}
& \mathrm{R} 23979 \text { : imazalil (base) } \\
& \mathrm{R}=\left(\mathrm{m}_{\mathrm{t}}-\mathrm{m}_{\mathrm{w}}\right) \mathrm{v}_{\mathrm{w}} / \mathrm{m}_{\mathrm{w}} \cdot \mathrm{v}_{\mathrm{O}} \\
& { }_{\mathrm{m}}{ }_{\mathrm{t}} \text { : total weight of } \mathrm{R} 23979 \\
& { }_{\mathrm{w}}^{\mathrm{w}} \text { : weight in aqueous phase } \\
& { }_{\mathrm{w}} \text { : volume of aqueous phase } \\
& { }_{\mathrm{v}} \text { : volume of octanol phase }
\end{aligned}
$$

Remark : the solubility of imazalil decreases at lower $\mathrm{pH}$. 


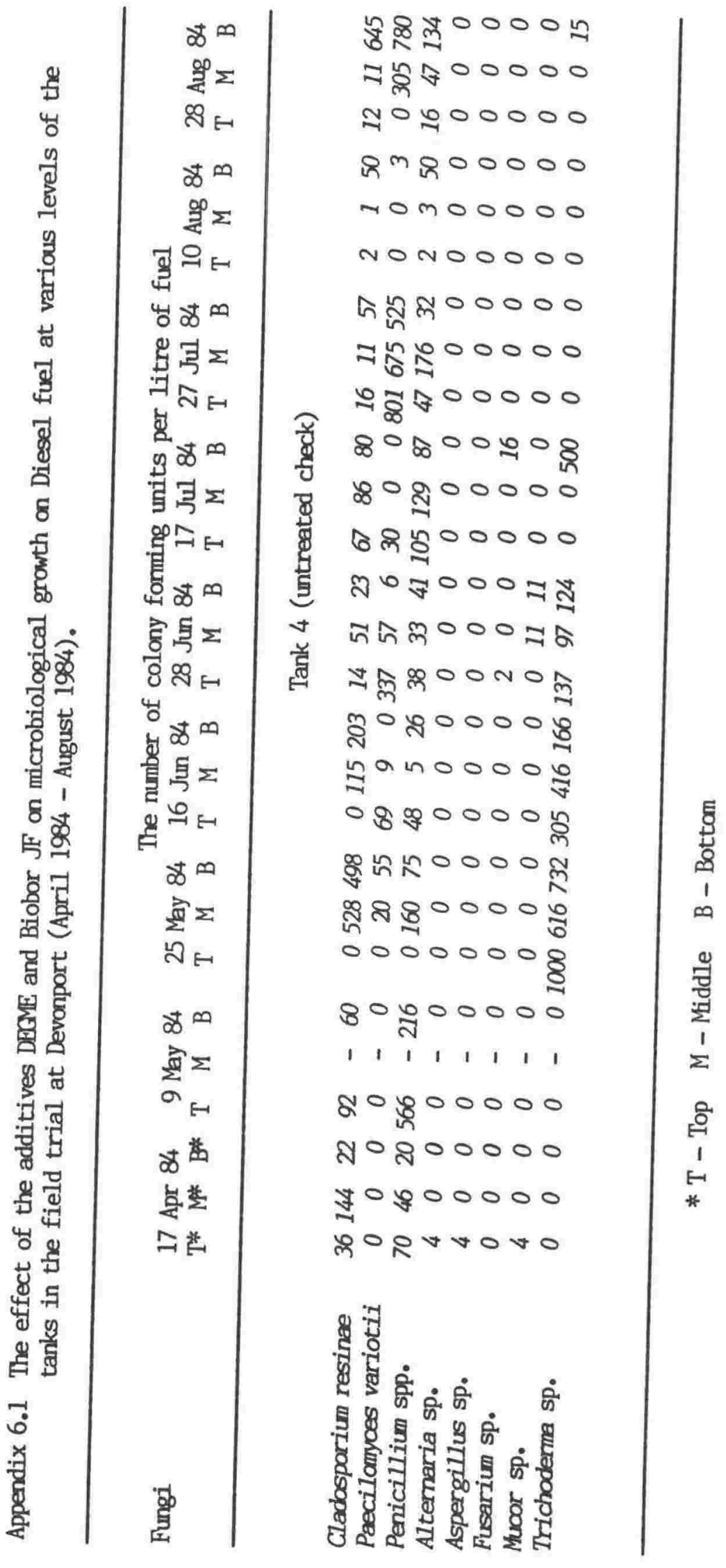




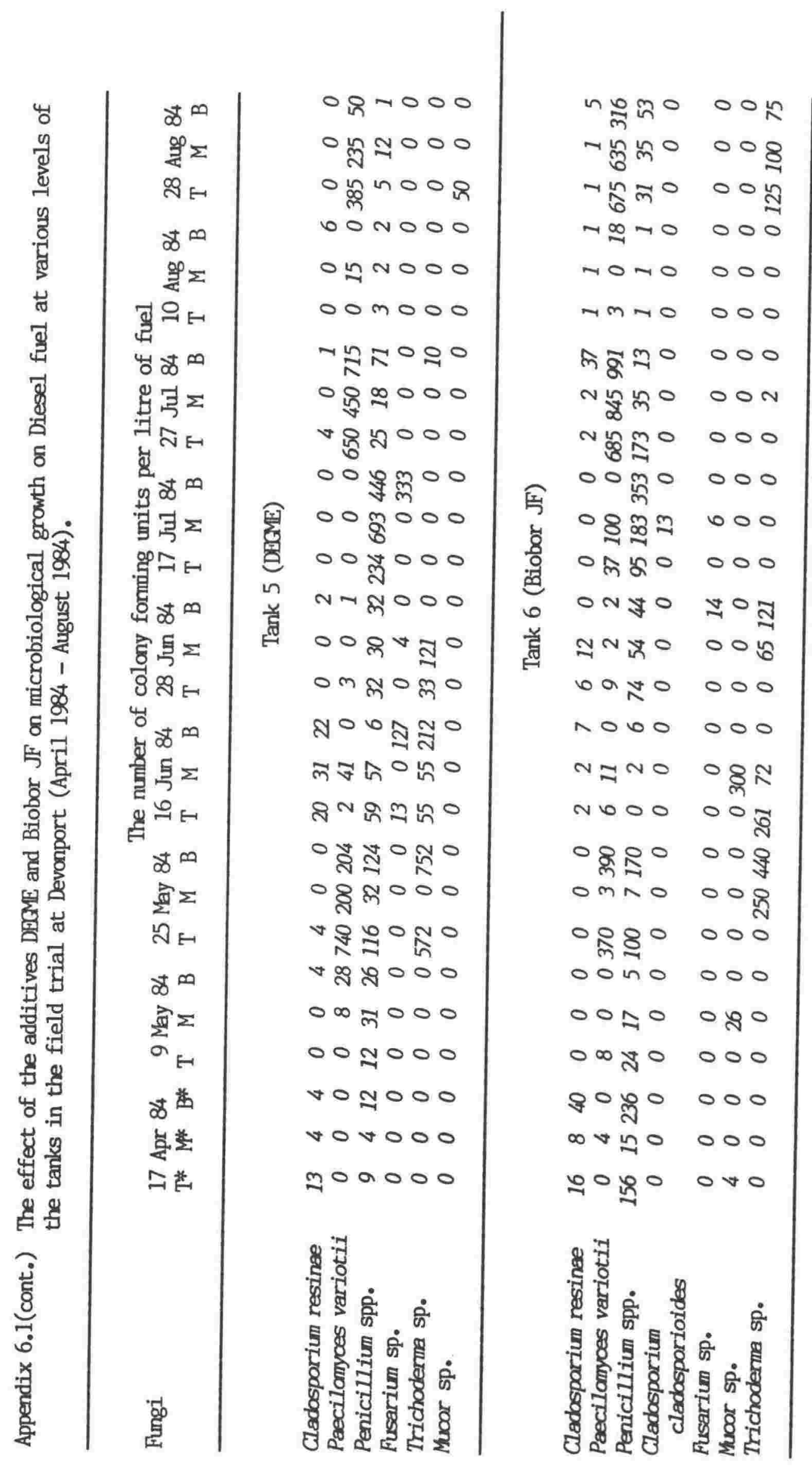




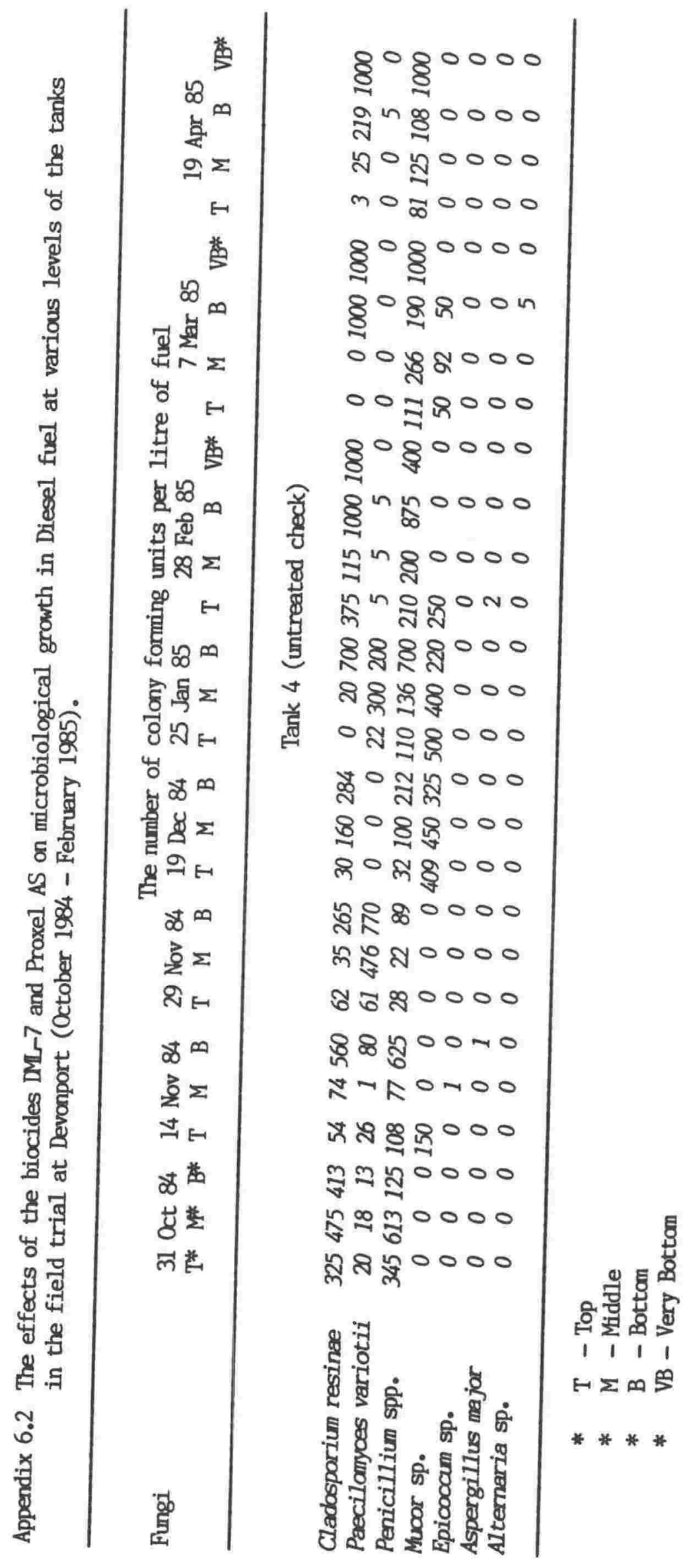




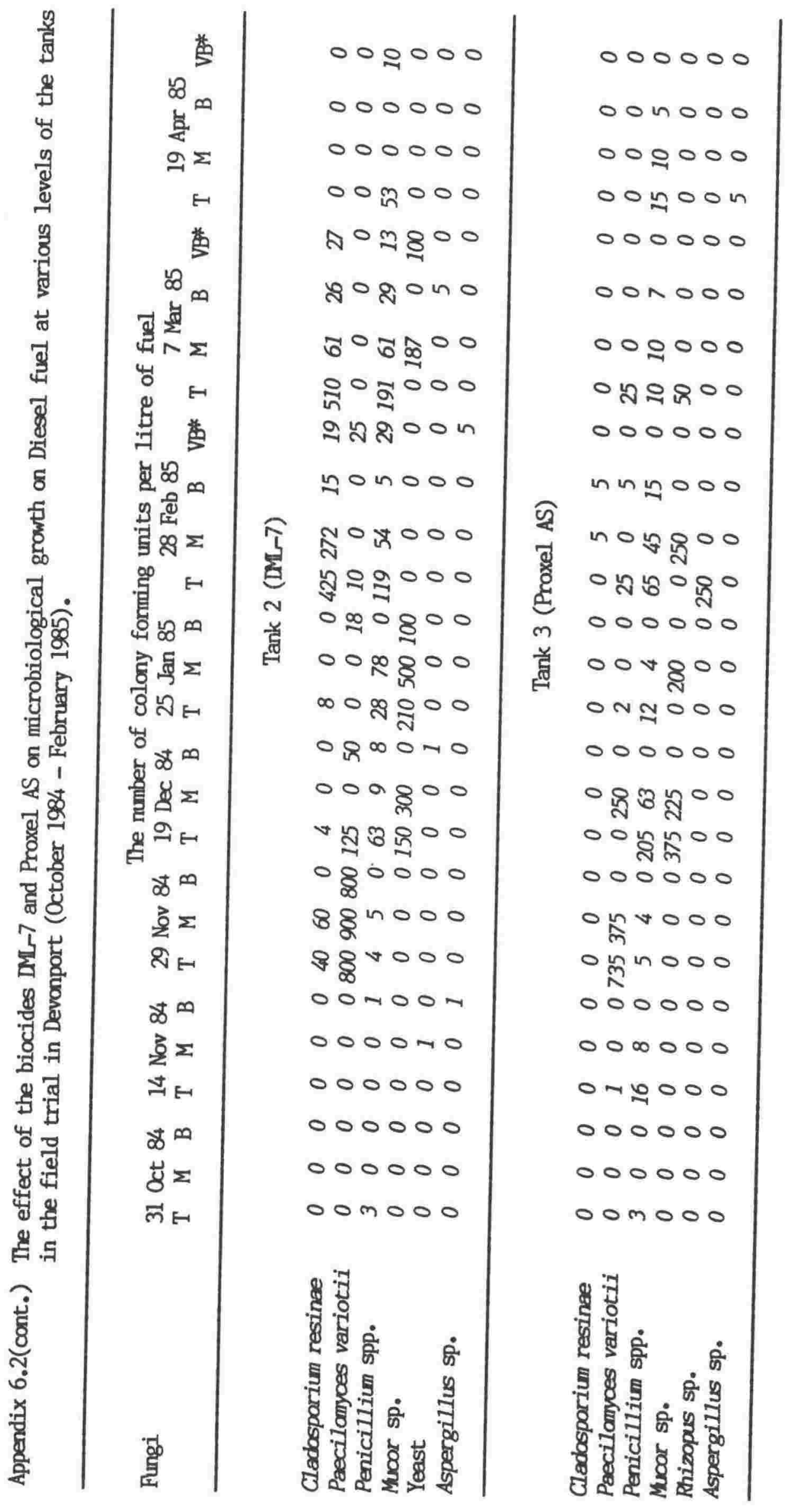


Appendix 6.3 The effects of the biocides DML-7 and Proxel AS on microbiological growth in Diesel fuel field trial (May 1985).
Fuel semple replication study.

Fungi

The number of colony forming units per litre of fuel

Top Middle Bottam

*R1 R2 R3 Mean R1 R2 R3 Mean R1 R2 R3 Mean

Tank 4 (untreated check)

Cladosporium resinae

Penicillium spp.

Trichoderma sp.

Paecilamyces variotii

Altemaria sp.

Epicoccum sp.

Mucar sp.

C. cladosporioides

Aspergillus niger

Fusarium sp.

Botrytis sp.

Aspergillus sp.

Pink Yeast

$\begin{array}{rrrrrrrrrrrr}12 & 0 & 0 & 4 & 3 & 1 & 0 & 1 & 130 & 600 & 223 & 318 \\ 34 & 276 & 6 & 105 & 181 & 318 & 62 & 187 & 130 & 415 & 655 & 400 \\ 5 & 0 & 0 & 2 & 0 & 0 & 0 & 0 & 0 & 0 & 0 & 0 \\ 141 & 13 & 0 & 51 & 111 & 14 & 8 & 44 & 0 & 2 & 5 & 2 \\ 1 & 3 & 0 & 1 & 0 & 0 & 0 & 0 & 0 & 0 & 0 & 0 \\ 1 & 0 & 0 & 1 & 0 & 0 & 0 & 0 & 0 & 0 & 0 & 0 \\ 0 & 200 & 0 & 67 & 0 & 0 & 150 & 50 & 0 & 0 & 0 & 0 \\ 0 & 0 & 1 & 1 & 2 & 0 & 0 & 1 & 0 & 0 & 0 & 0 \\ 0 & 0 & 1 & 1 & 1 & 1 & 0 & 1 & 0 & 0 & 0 & 0 \\ 0 & 0 & 0 & 0 & 0 & 2 & 0 & 1 & 0 & 0 & 0 & 0 \\ 0 & 0 & 0 & 0 & 0 & 0 & 1 & 1 & 0 & 0 & 0 & 0 \\ 0 & 0 & 0 & 0 & 0 & 0 & 0 & 0 & 1 & 0 & 0 & 1 \\ 0 & 0 & 0 & 0 & 0 & 0 & 0 & 0 & 0 & 1 & 0 & 1\end{array}$

Tank 2 (DM-7)

Cladosporium resinae

Penicillium spp.

Paecilamyces variotii

Mucor sp.

Alternaria sp.

$$
\begin{array}{rrrrrrrrrrrr}
0 & 2 & 0 & 1 & 0 & 0 & 0 & 0 & 0 & 1 & 2 & 2 \\
1 & 10 & 7 & 6 & 11 & 3 & 3 & 6 & 0 & 15 & 0 & 5 \\
2 & 0 & 0 & 1 & 0 & 0 & 0 & 0 & 5 & 43 & 6 & 18 \\
0 & 0 & 0 & 0 & 100 & 0 & 0 & 33 & 100 & 100 & 0 & 67 \\
0 & 0 & 0 & 0 & 0 & 3 & 0 & 2 & 0 & 0 & 0 & 0
\end{array}
$$

Tank 3 (Proxel AS)

$\begin{array}{lrrrrrrrrrrrr}\text { Cladosporium resinae } & 0 & 0 & 2 & 1 & 0 & 0 & 0 & 0 & 0 & 0 & 0 & 0 \\ \text { Penicillium spp. } & 11 & 17 & 11 & 13 & 6 & 6 & 4 & 5 & 8 & 5 & 2 & 5 \\ \text { Paecillamyces variotii } & 1 & 0 & 0 & 1 & 0 & 0 & 0 & 1 & 0 & 1 & 1 & 1 \\ \text { Trichoderma sp. } & 0 & 400 & 0 & 133 & 0 & 0 & 0 & 0 & 200 & 200 & 210 & 203 \\ \text { Mucor sp. } & 300 & 100 & 0 & 133 & 400 & 400 & 200 & 333 & 0 & 0 & 0 & 0 \\ \text { Rhizopus sp. } & 0 & 200 & 300 & 167 & 0 & 0 & 0 & 0 & 0 & 0 & 0 & 0 \\ \text { Aspergillus niger } & 0 & 1 & 0 & 1 & 0 & 0 & 0 & 0 & 0 & 0 & 0 & 0 \\ \end{array}$




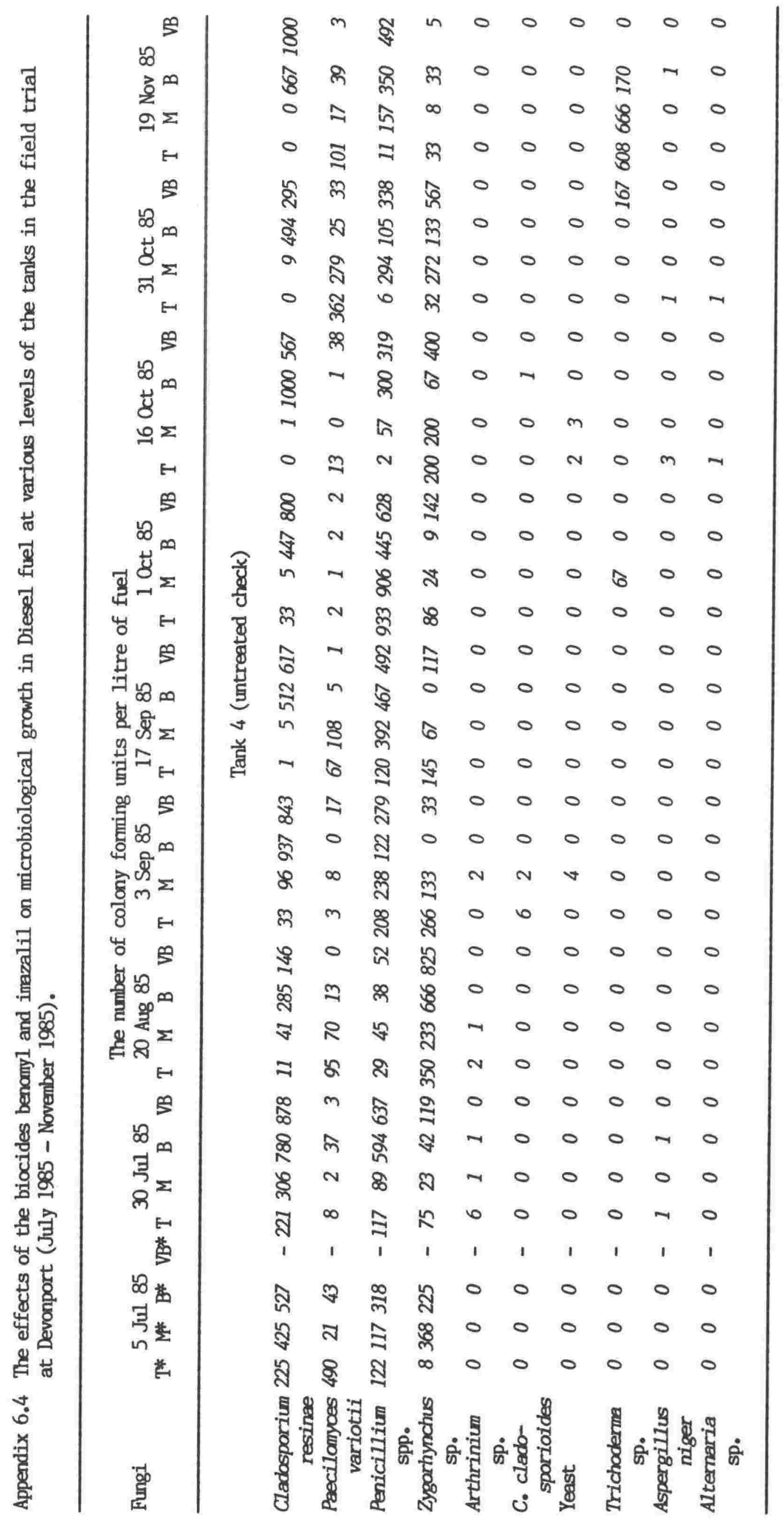




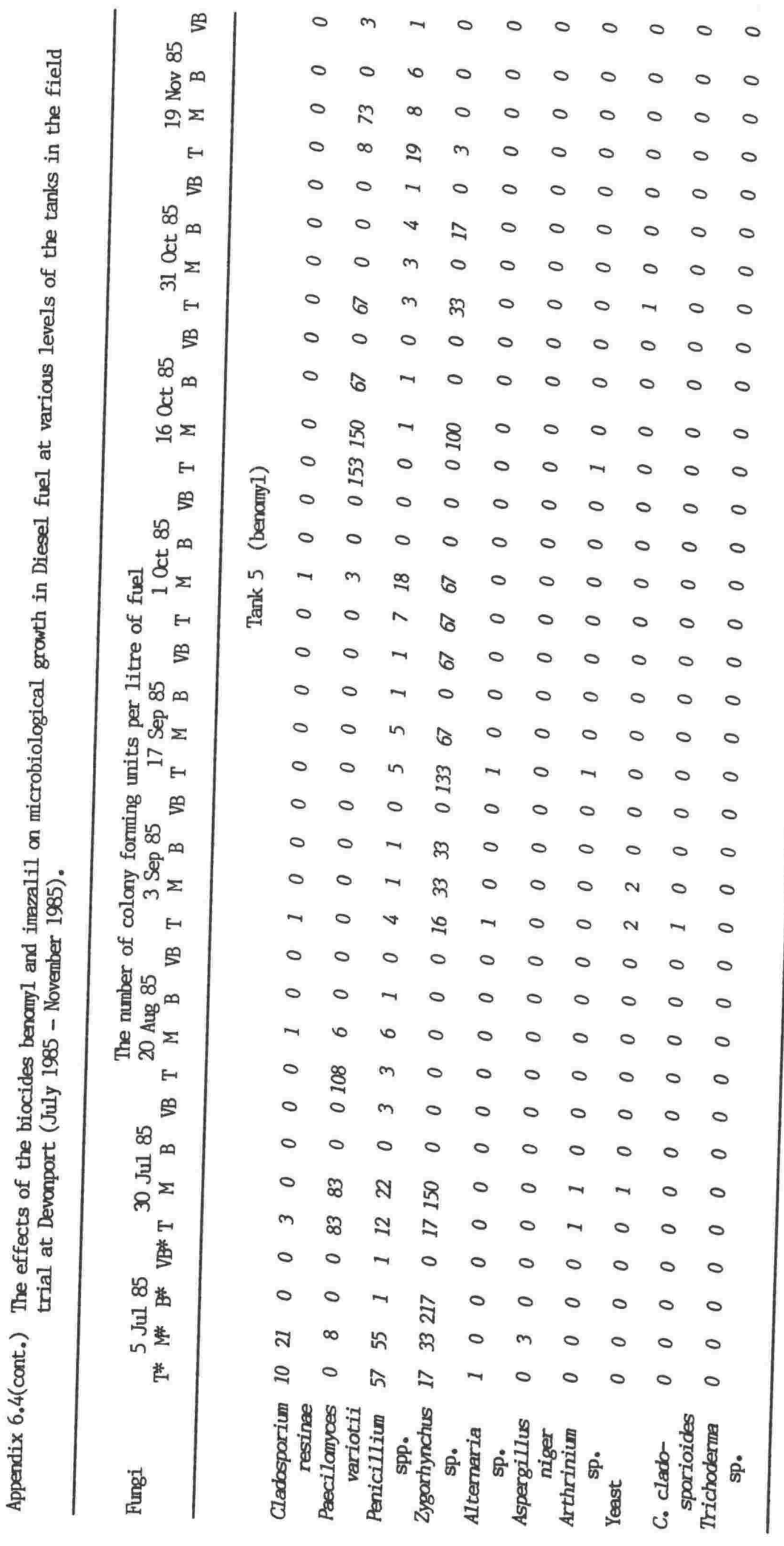




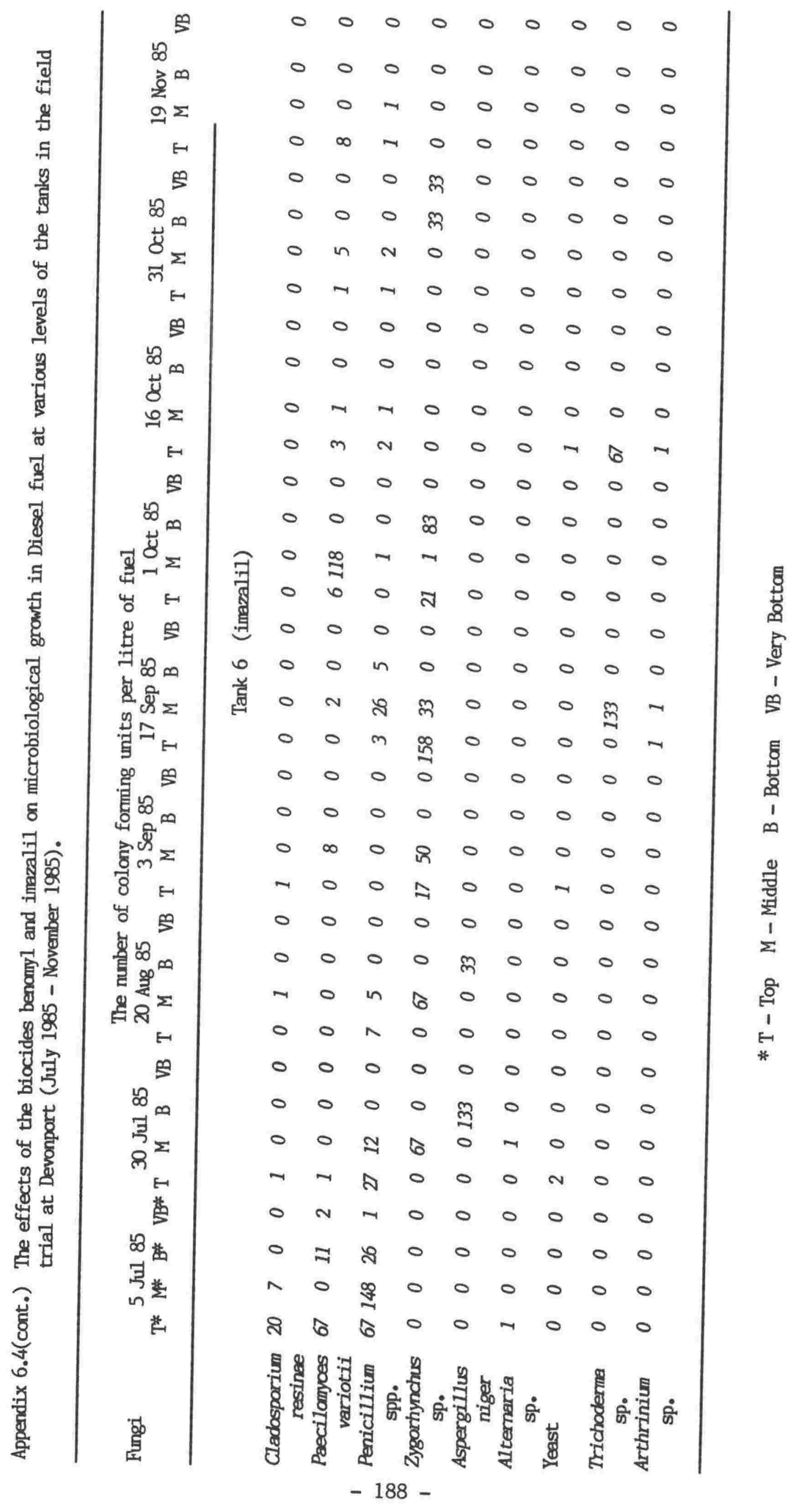


Appendix 6.5 The effects of the biocides benomyl and imazalil on microbiological growth in Diesel fuel field trial (July 1985 - November 1985).

Fuel sample replication study.

Fungi

The number of colony forming units per litre of fuel

Top

Middle

Bottom

Very Bottan

*R1 R2 R3 Mean R1 R2 R3 Mean R1 R2 R3 Mean R1 R2 R3 Mean

Tank 4 (untreated check)

Cladosporium resinae

Penicillium spp.

Paecilamyces variotii

Zygorhynchus sp.

Arthrinium sp.

Aspergillus niger

Alternaria

C. cladosporioides

Trichoderma sp.

Yeast $\begin{array}{llllllllllllllll}58 & 54 & 43 & 52 & 70 & 158 & 21 & 28 & 624 & 667 & 527 & 606 & 634 & 664 & 631 & 643\end{array}$

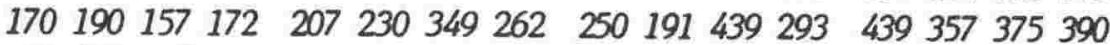

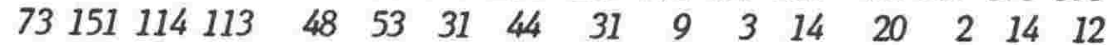

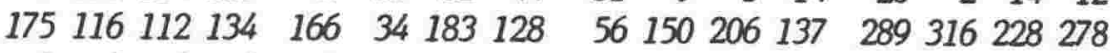

$\begin{array}{llllllllllllllll}2 & 2 & 1 & 2 & 1 & 1 & 1 & 1 & 1 & 1 & 0 & 1 & 0 & 0 & 0 & 0\end{array}$

$\begin{array}{llllllllllllllll}1 & 1 & 1 & 1 & 0 & 0 & 0 & 0 & 2 & 0 & 0 & 1 & 0 & 1 & 0 & 1\end{array}$

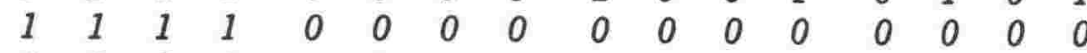

$\begin{array}{llllllllllllllll}0 & 2 & 0 & 1 & 0 & 2 & 0 & 1 & 0 & 0 & 0 & 0 & 0 & 0 & 0 & 0\end{array}$

$\begin{array}{llllllllllllllll}56 & 81 & 67 & 68 & 133 & 89 & 22 & 81 & 0 & 22 & 34 & 19 & 0 & 38 & 25 & 21\end{array}$

Tank 5 (Benomyl treated)

$\begin{array}{lrrrrrrrrrrrrrrrr}\text { Cladosporium resinae } & 2 & 2 & 1 & 2 & 2 & 2 & 3 & 2 & 0 & 0 & 0 & 0 & 0 & 0 & 0 & 0 \\ \text { Penicillium spp. } & 13 & 14 & 10 & 12 & 13 & 11 & 15 & 4 & 1 & 2 & 1 & 1 & 1 & 1 & 1 & 1 \\ \text { Paecilamyces variotii } & 46 & 40 & 54 & 47 & 31 & 53 & 25 & 36 & 0 & 1 & 22 & 8 & 0 & 0 & 0 & 0 \\ \text { Zygorhynchus sp. } & 0 & 50 & 45 & 32 & 45 & 78 & 28 & 50 & 33 & 28 & 28 & 21 & 22 & 1 & 0 & 8 \\ \text { Alternaria sp. } & 2 & 3 & 0 & 2 & 1 & 0 & 0 & 1 & 1 & 0 & 0 & 1 & 0 & 0 & 0 & 0 \\ \text { Aspergillus niger } & 0 & 0 & 0 & 0 & 1 & 0 & 0 & 1 & 0 & 0 & 0 & 0 & 0 & 0 & 0 & 0 \\ \text { Arthrinium sp. } & 2 & 1 & 2 & 2 & 1 & 1 & 0 & 1 & 0 & 0 & 0 & 0 & 0 & 0 & 0 & 0 \\ \text { Yeast } & 1 & 1 & 1 & 1 & 1 & 1 & 0 & 1 & 0 & 0 & 0 & 0 & 0 & 0 & 0 & 0 \\ \text { C. cladosporioides } & 1 & 0 & 0 & 1 & 0 & 0 & 0 & 0 & 0 & 0 & 0 & 0 & 0 & 0 & 0 & 0 \\ \text { Trichoderma sp. } & 0 & 0 & 11 & 4 & 0 & 0 & 0 & 0 & 0 & 0 & 0 & 0 & 0 & 0 & 0 & 0 \\ & & & & & & & & & & & & \end{array}$

Tank 6 (Imazalil treated)

$\begin{array}{lrrrrrrrrrrrrrrrr}\text { Aspergillus niger } & 0 & 0 & 0 & 0 & 0 & 0 & 11 & 4 & 0 & 0 & 0 & 0 & 0 & 0 & 0 & 0 \\ \text { Cladosporium resinae } & 7 & 2 & 0 & 3 & 0 & 2 & 0 & 1 & 0 & 0 & 0 & 0 & 0 & 0 & 0 & 0 \\ \text { Penicillium spp. } & 26 & 6 & 4 & 12 & 30 & 33 & 1 & 21 & 2 & 9 & 1 & 4 & 0 & 0 & 3 & 1 \\ \text { Paecilamyces variotii } & 3 & 3 & 22 & 8 & 1 & 7 & 37 & 15 & 0 & 4 & 0 & 1 & 0 & 0 & 3 & 1 \\ \text { Zygarhynchus sp. } & 24 & 31 & 11 & 22 & 14 & 22 & 14 & 17 & 22 & 33 & 28 & 28 & 11 & 0 & 6 & 6 \\ \text { Arthrinium sp. } & 1 & 4 & 0 & 2 & 0 & 1 & 0 & 1 & 0 & 0 & 0 & 0 & 0 & 0 & 0 & 0 \\ \text { Trichoderma sp. } & 0 & 0 & 22 & 7 & 0 & 44 & 0 & 15 & 0 & 0 & 0 & 0 & 0 & 0 & 0 & 0 \\ \text { Alternaria sp. } & 1 & 0 & 0 & 1 & 1 & 0 & 0 & 1 & 0 & 0 & 0 & 0 & 0 & 0 & 0 & 0 \\ \text { Yeast } & 1 & 4 & 3 & 3 & 1 & 0 & 0 & 1 & 0 & 0 & 0 & 0 & 0 & 0 & 0 & 0\end{array}$




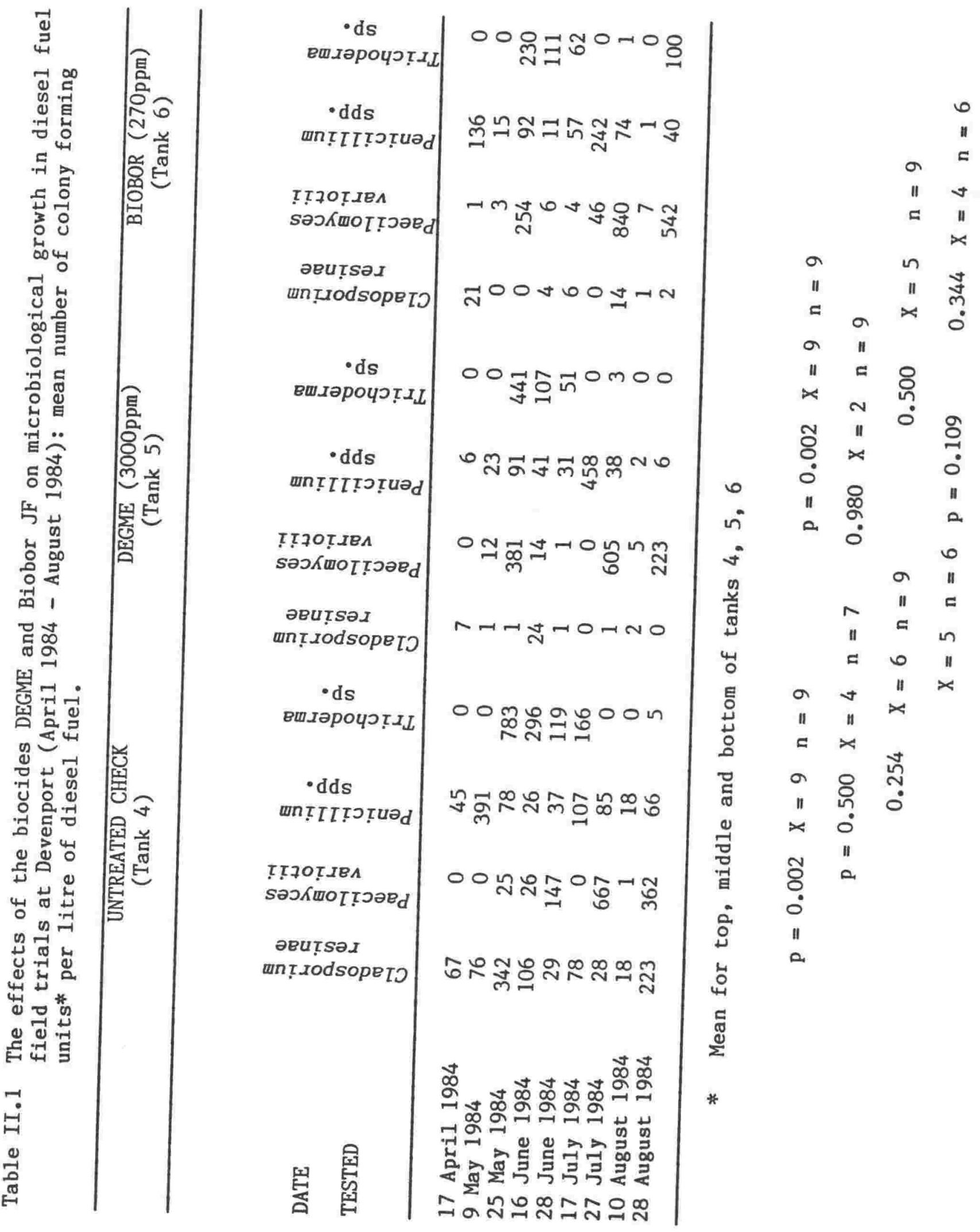




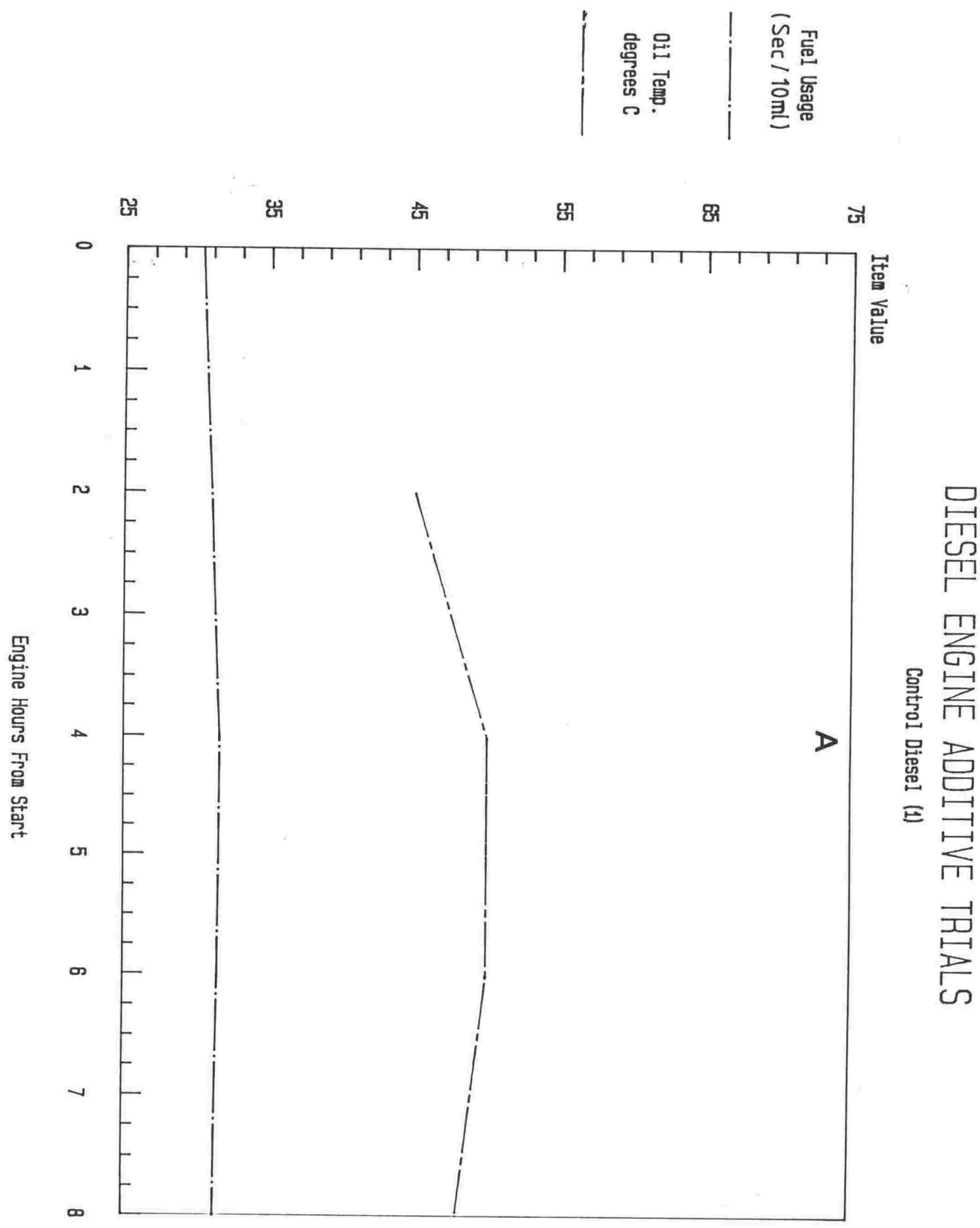




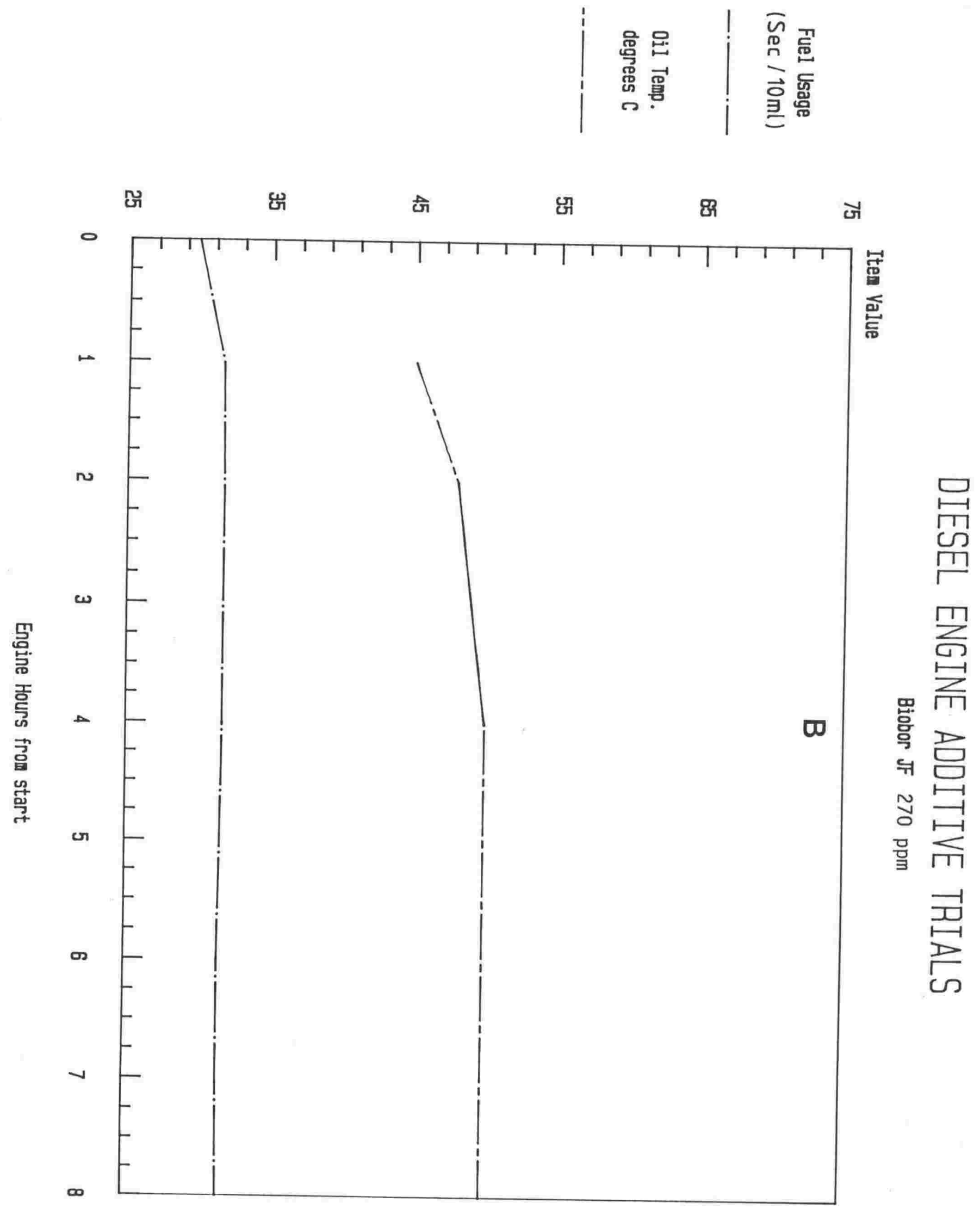




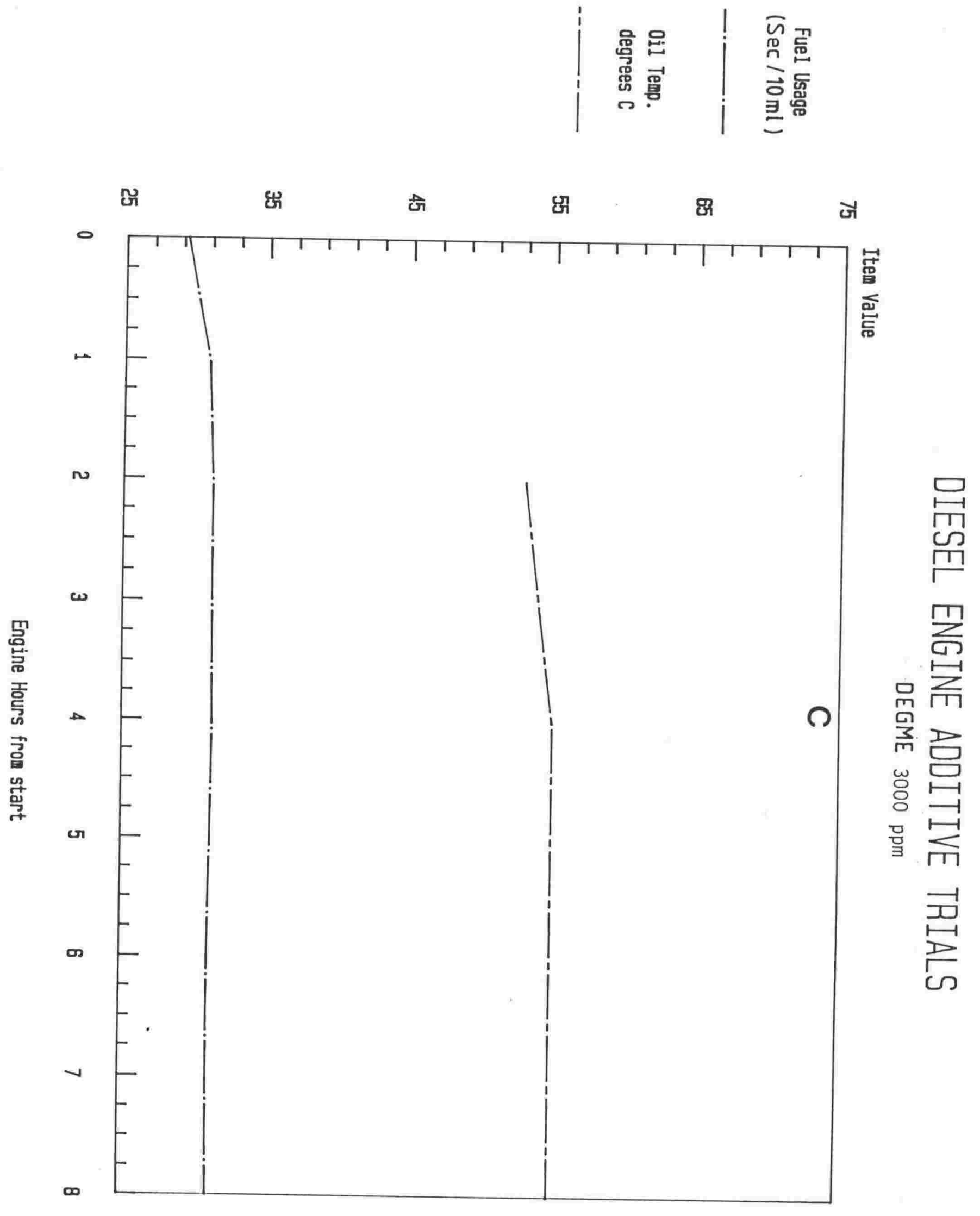




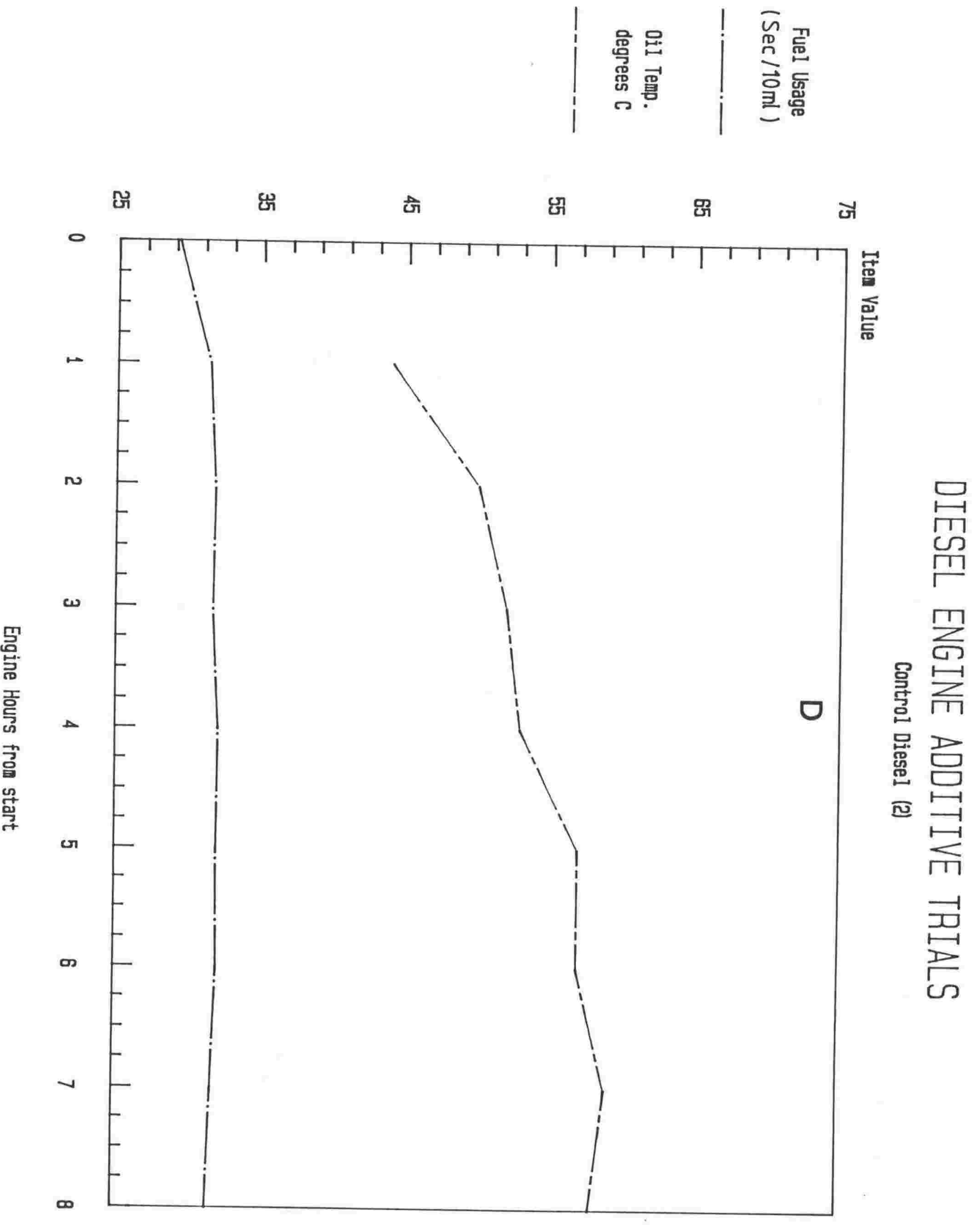



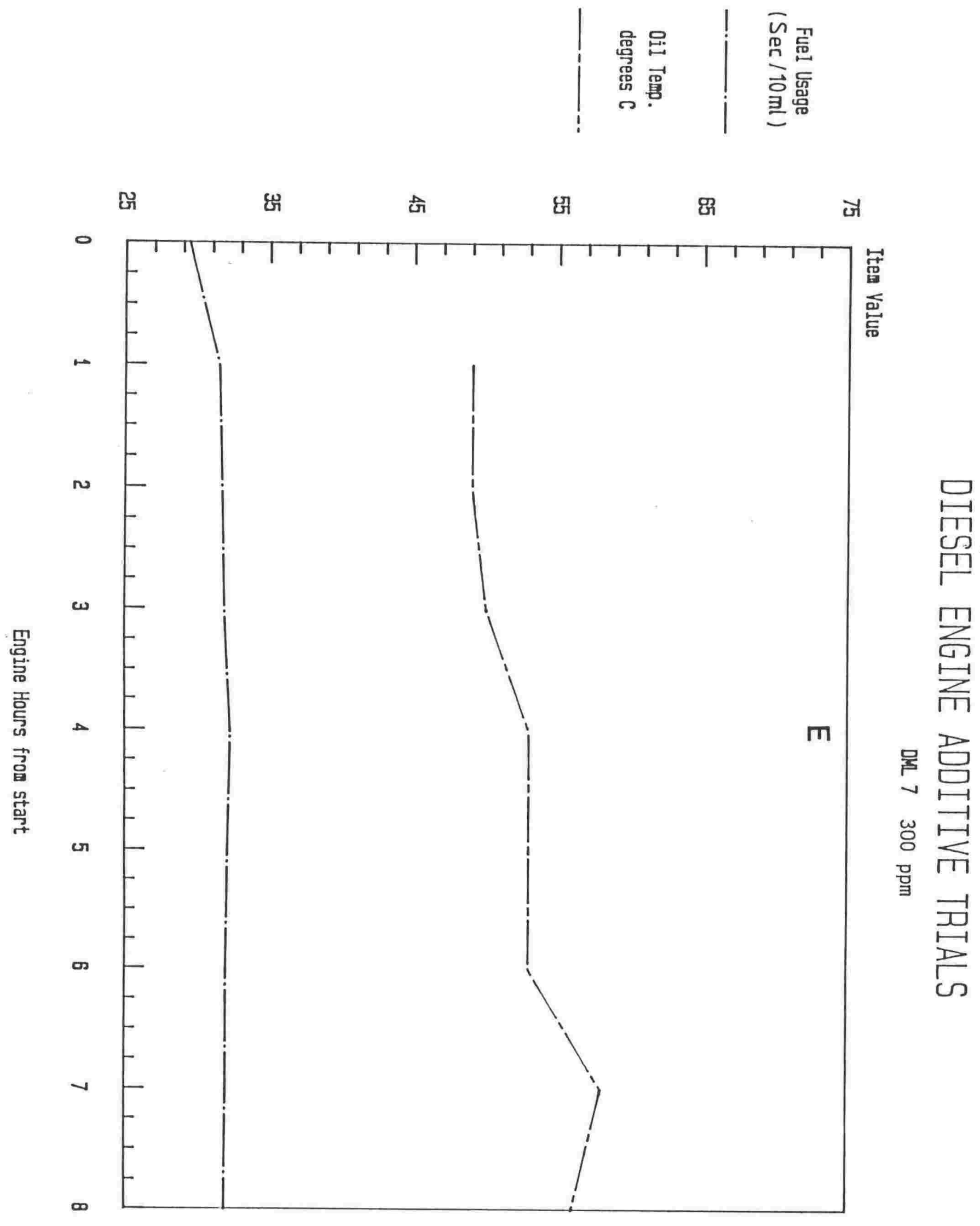


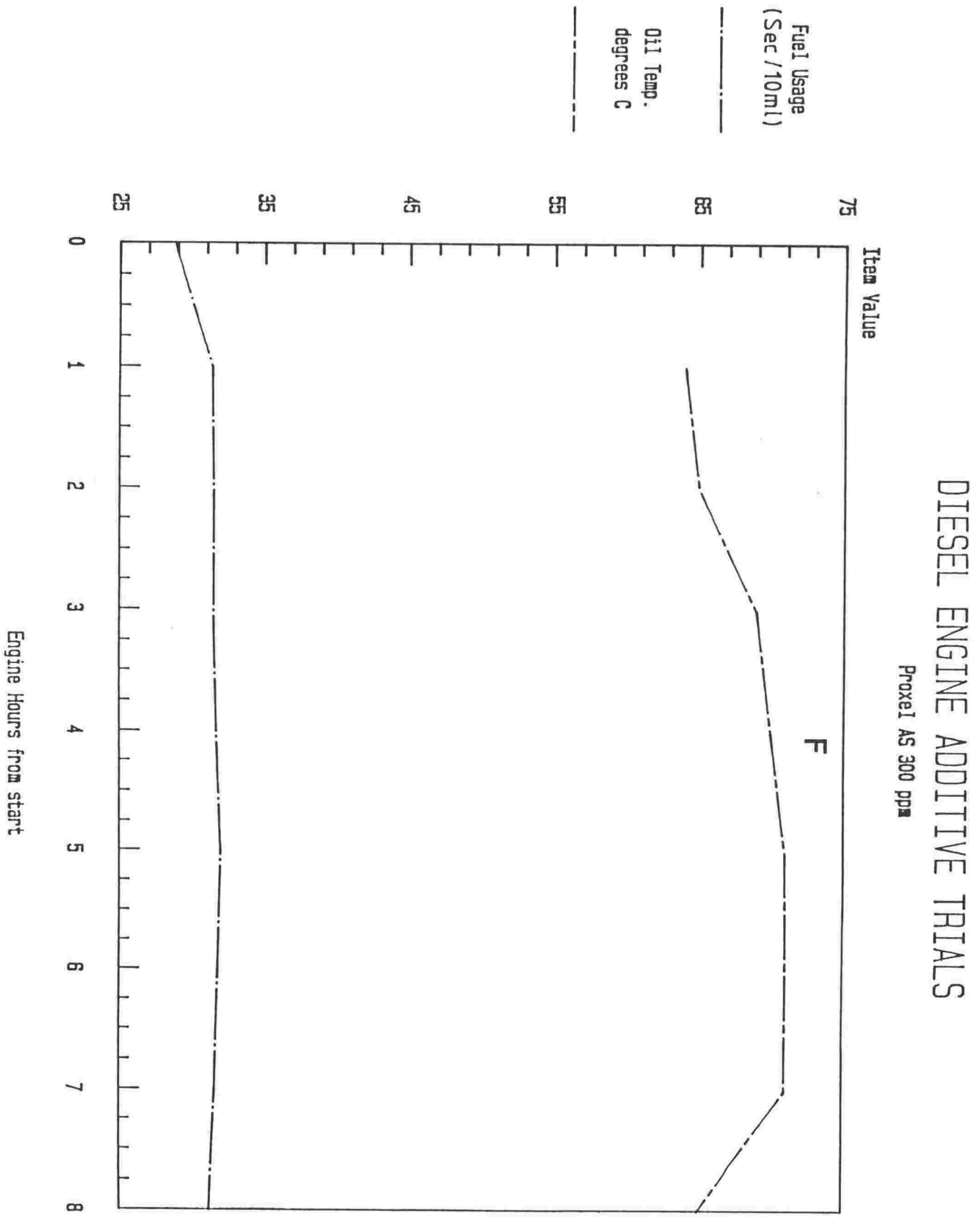



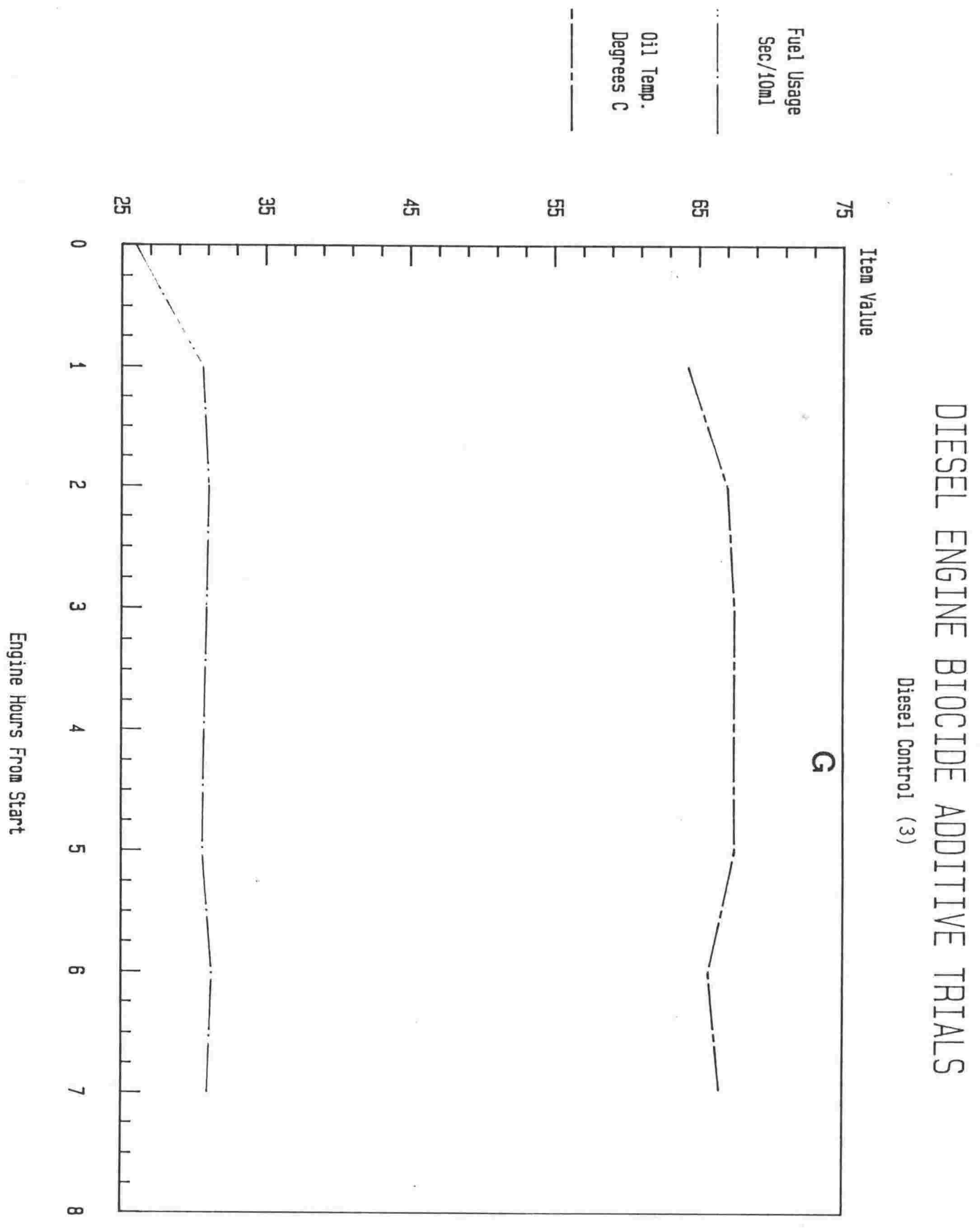


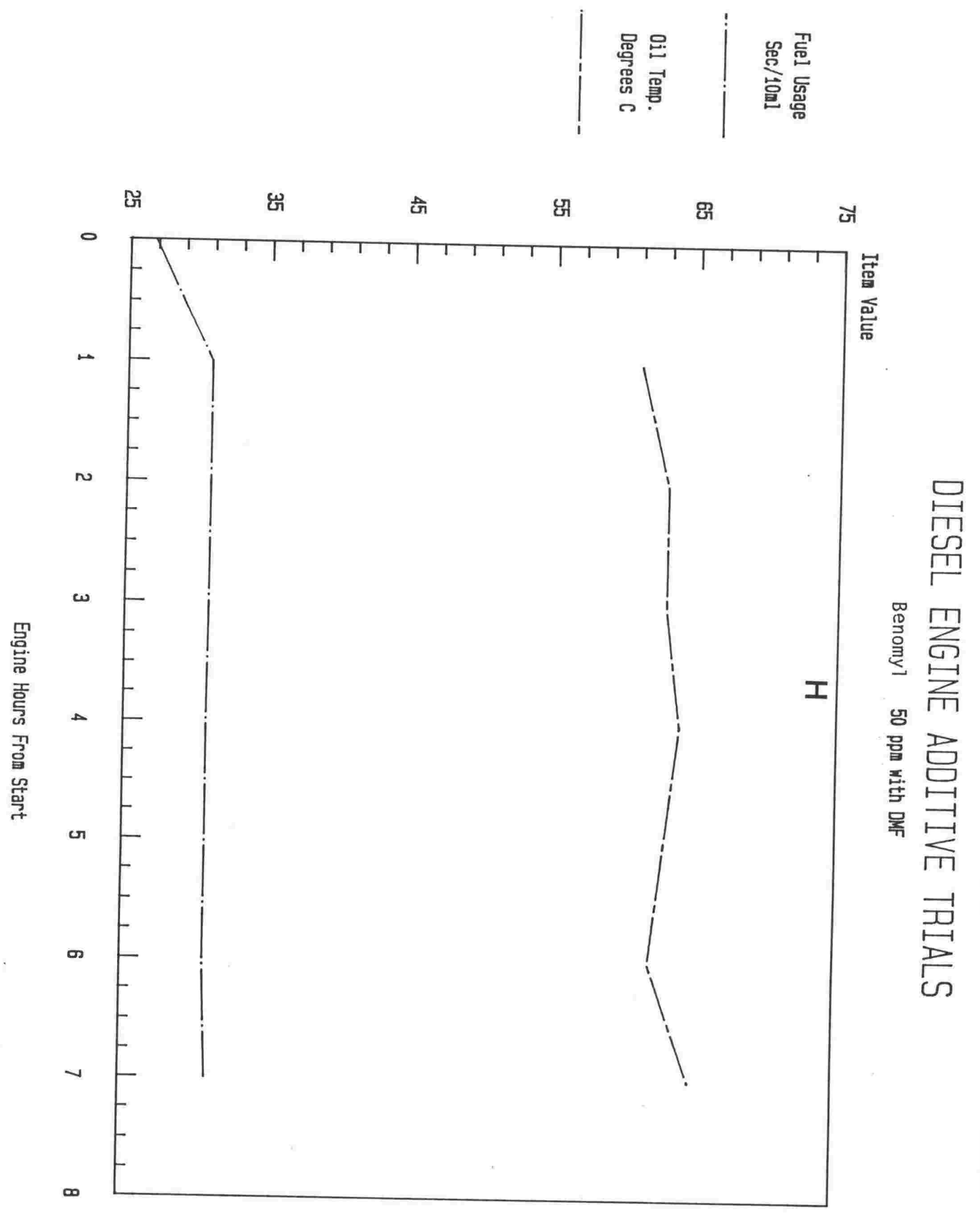




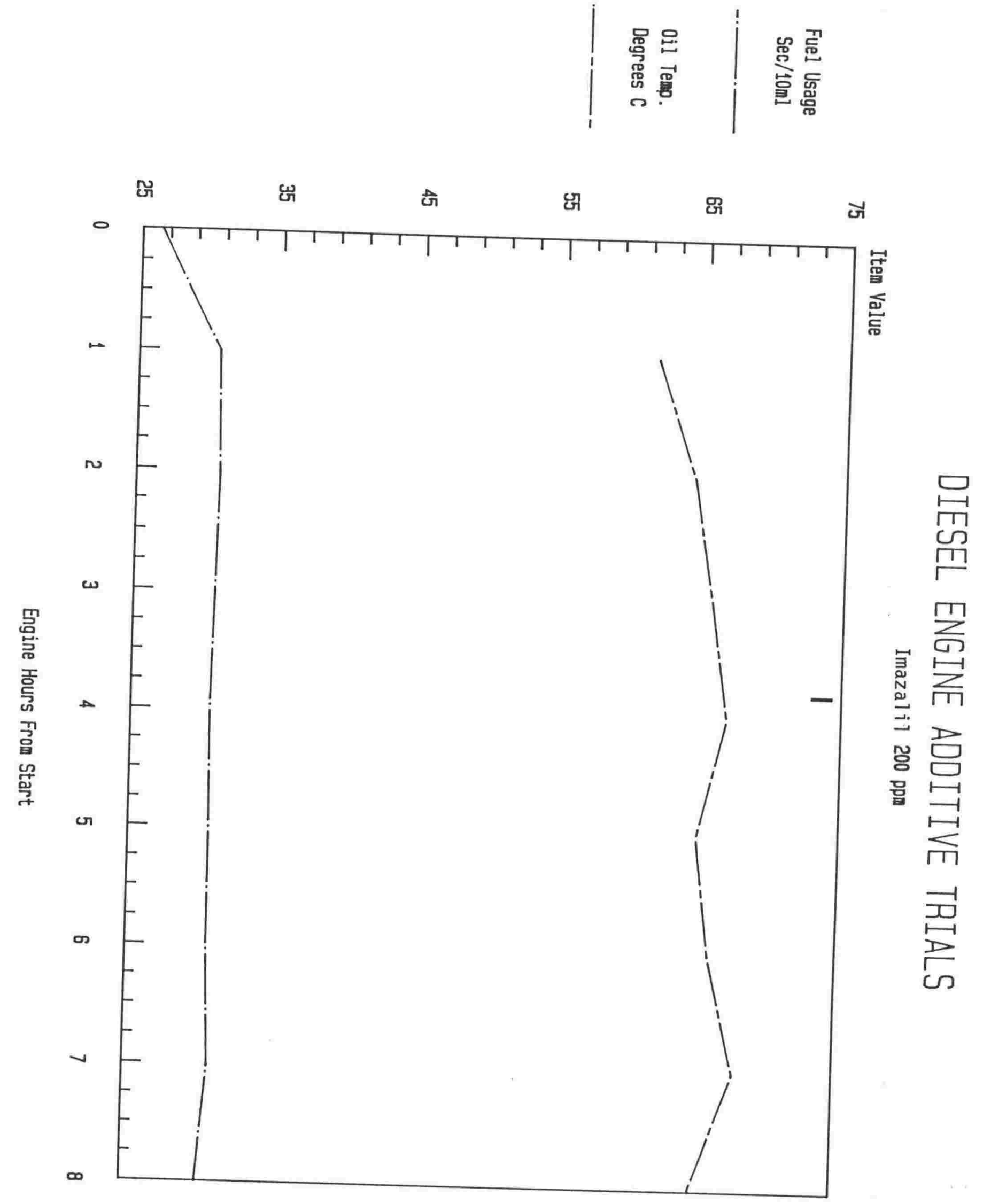




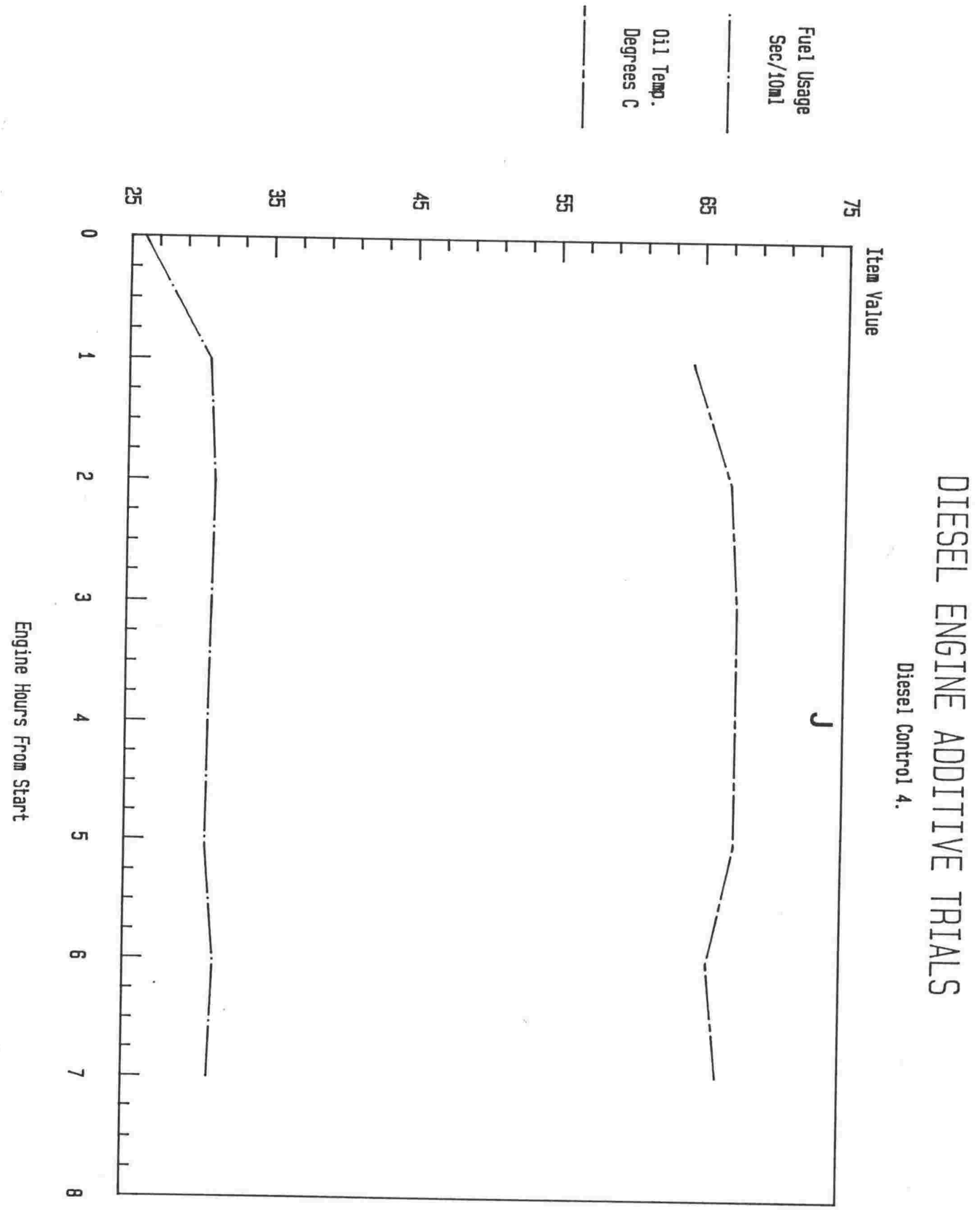




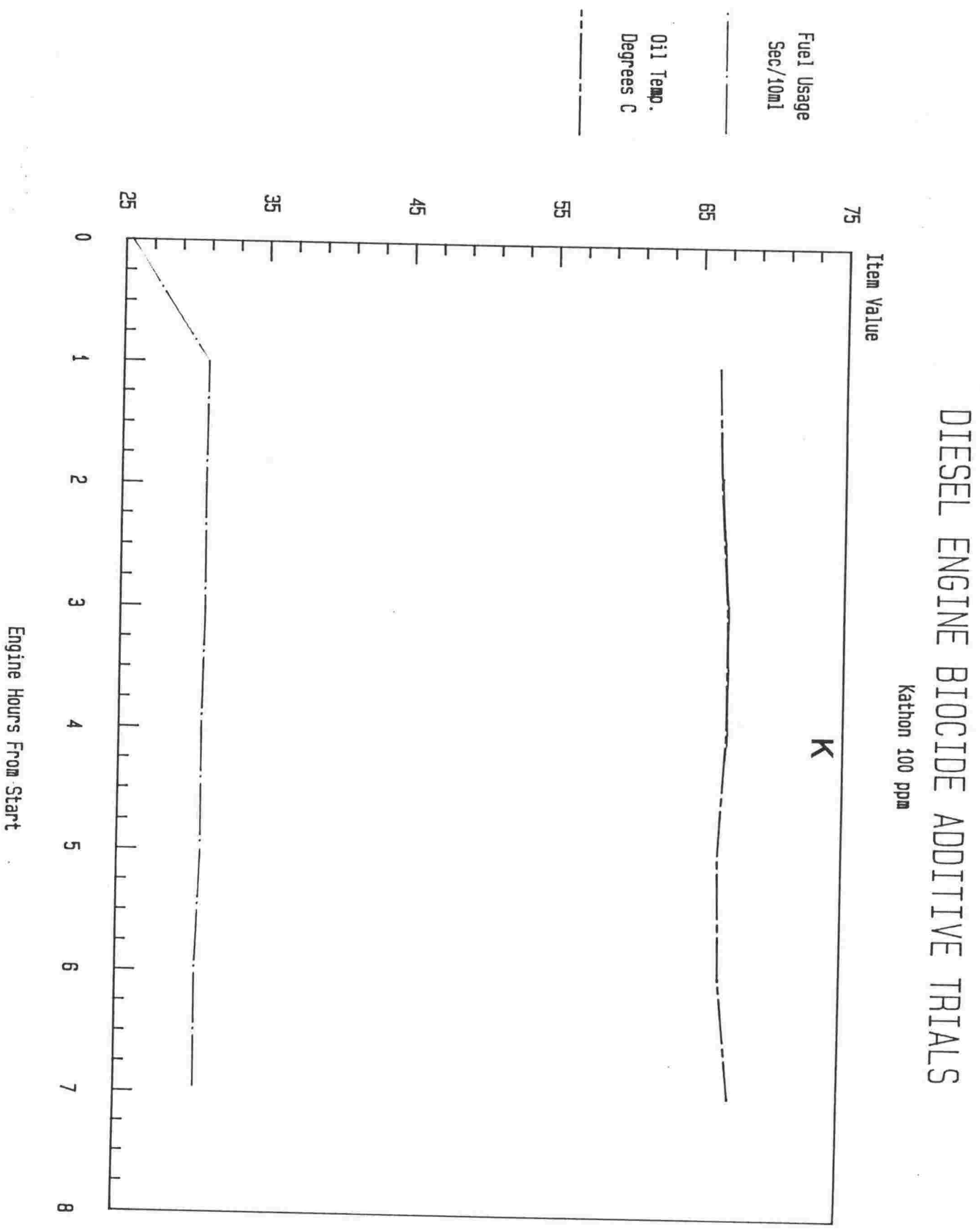



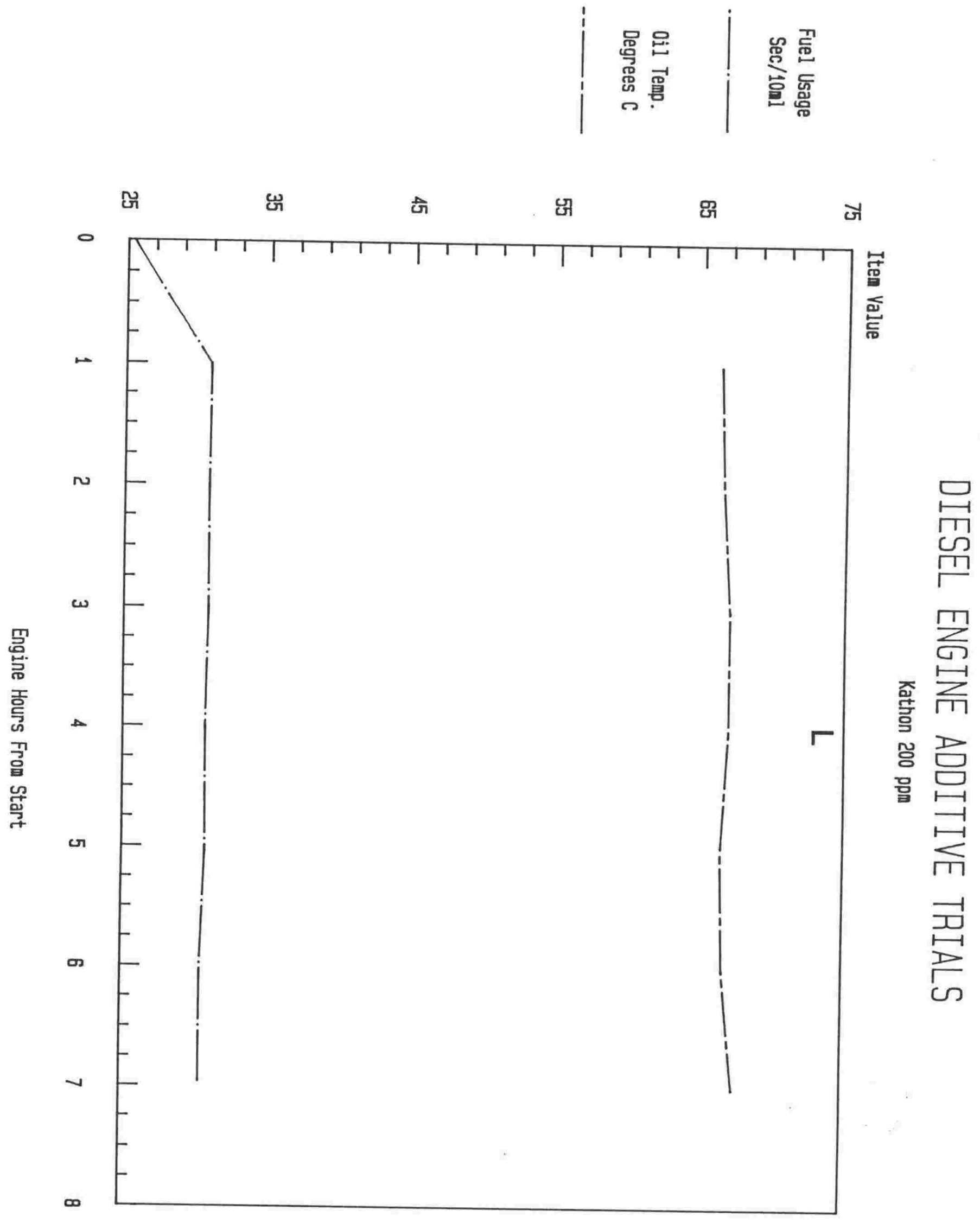
1. DESCRIPTION OF THE THREE MAIN CONTAMINATING FUNGI OF DIESEL FUEL ISOLATED IN THIS STUDY

Cladosporium resinae (Lindau) de Vries = Hormoconis resinae (Lindau)

V. Arx \& de Vries comb. nov. Teleomorph: Amorphotheca resinae Parbery.

Colonies on malt extract agar (MEA) plates, after 5 days at $25^{\circ} \mathrm{C}$, powdery, due to profuse sporulation with little aerial mycelium, hazel to brown in colour with a white margin.

Conidiophores up to $2 \mathrm{~mm}$ long, 2.5 - $6 \mu \mathrm{m}$ thick, smooth. Ramoconidia, when present, clavate or cylindrical, $8-20 \mu \mathrm{m}$ 1ong, $3-7 \mu \mathrm{m}$ thick, smooth. Conidia borne on ramoconidia solitary or in chains of rarely more than 3 conidia, not separated by scars, broadly ellipsoidal or ovoid, 0 septate, brown or olivaceous brown, smooth, common1y $3-6 \times 2$ $-3.5 \mu \mathrm{m}$.

Three other forms have been described (Sheridan, Tan and Nelson, 1972) but only $f$. avellaneum was isolated from diesel fuel in this study.

It has been found that four carboxylic acids were produced by $C$. resinae and identified as dodecanoic, acetic, glycolic and glyoxylic acids (Siporin and Cooney, 1975), which are corrosive to aluminium and its alloy.

Paecilomyces variotii Bainier. Teleomorphs: Byssochlamys, Thermoascus, Talaromyces. Colonies on MEA floccose appearance, usually coloured uniformly brown or olive brown from conidia; reverse pale.

Penicilli borne from aerial hyphae on short stfipes of irregular pattern, a cluster of phialides alone or with metulae and phialides; phialides 12 - $20 \mu \mathrm{m}$ 1ong, tapering gradually. Conidia mostly subspheroidal to ellipsoidal, sometimes cylindroidal or pyriform, usually 3.0 - $5.0 \mu \mathrm{m}$ long, smooth-walled. 
P. variotii was said to stimulate the growth of barley seedlings. Contaminated grain proved to be toxic to young ducklings. As the causal agent of paecilomycosis it has been isolated from a generalised infection in a dog, an aborted calf, mycotic stomach ulcer in a calf, kidney infection of a horse, from brain and lung of a turkey and a rat caecum (Domsch, Gams and Anderson, 1980).

Penicillium corylophilum Dierckx.

Colonies on MEA strictly velutinous, mycelium white. Conidiogenesis moderate, dull green, clear exudate occasionally present, reverse pale at the margins, but usually dull green to very dark green centrally. Conidiophores borne from subsurface hyphae, stipes 100 - $250 \mu \mathrm{m}$ long; rough-walled. Penicilli with two metulae the offset one often longer than the axial, phialides ampulliform, 7 - $11 \mu \mathrm{m}$ long; conidia spherical to subspheroidal, commonly 2.5 - $3.0 \mu \mathrm{m}$ diameter, smoothwalled.

P. corylophilum was reported to degrade starch and grows on media with $5 \%$ tannin or on substrates with hexadecane, dodecane, octadecane, cyclohexane, toluene, benzene or kerosene as sole $\mathrm{C}$ source in the presence of yeast extract. It produces D-xylonic acid, ethylene and viridicatin-related metabolite (Domsch, Gams and Anderson, 1980).

This is the first study to establish that Paecilomyces variotii and Penicillium corylophilum grow in diesel at the fuel/water interface. Due to the limitation in time, no attempts were made to study the metabolites of $P$. variotii and Penicillium corylophilum and this could be a major study by itself. C. resinae degrades hydrocarbons and its metabolites has been widely studied.

\section{PH DATA ON THE FUNGAL GROWTH STUDIES}

C. resinae grew at the seawater/diesel fuel interface after $P$. corylophilum had been grown with an accompanying decrease in $\mathrm{pH}$ of about $3 \mathrm{pH}$ units ( 8.3 to 8 ). It is clear from these results that the normal pH of seawater (8.3) was too high to allow growth of $C$.

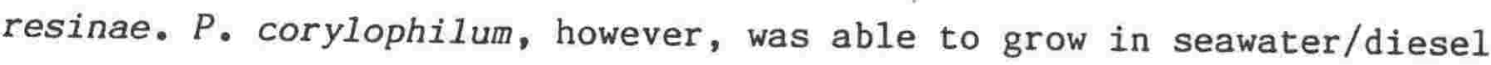
fuel medium. 
The reduction in $\mathrm{pH}$ accompanying the growth of $P$. corylophilum, appeared to be the major factor in promoting the growth of $C$. resinae.

The $\mathrm{pH}$ of $\mathrm{BH} / \mathrm{diesel}$ fuel, distilled water/diesel fuel, tapwater/diesel fuel remained neutral ( $\mathrm{pH} 7$ ) during and after the growth of the single and mixed cultures. There was no marked change in $\mathrm{pH}$.

\section{STATISTICAL ANALYSES}

Some of the problems encountered were:-

a) Impossibility of getting large number of multiple fuel samples from Auckland.

b) Irregularity in the fuel transport from Auckland to Wellington.

c) Fluctuation in the number of colony forming units even when continuous replicate samples were taken from each level at the same time.

Considering the above factors, statistical analysis may have distorted the raw data. -

According to Mr. R.M. Renner (Statistician), statistical modelling was not used in this study because the distributions of the colony-forming units controlling for concentration and other explanatory variables, were not known and too erratically skewed to suggest plausible transformations. Further, certain main effects that were observed appeared to be non-linear and convex, precluding monotonic transformations. Certain non-parametric tests were appropriate and consequently employed (see page 190). 
$\underline{\text { Reference }}$

Domsch, K.H., Gams, W., and Anderson, Traute-Heidi. 1980.

Compendium of soil fungi. Volume I. Academic Press, London.

Sheridan, J,E., Tan, Y.L., and Nelson, J. 1972. Studies on the

'kerosene fungus' Cladosporium resinae (Lindau) De Vries. Tuatara 19:130-165.

Siporin, C., and Cooney, J.J. 1975. Extracellular 1ipids of Cladosporium (Amorphotheca) resinae grown on glucose or on n-alkanes. Applied Microbiology 39(5):604-609. 

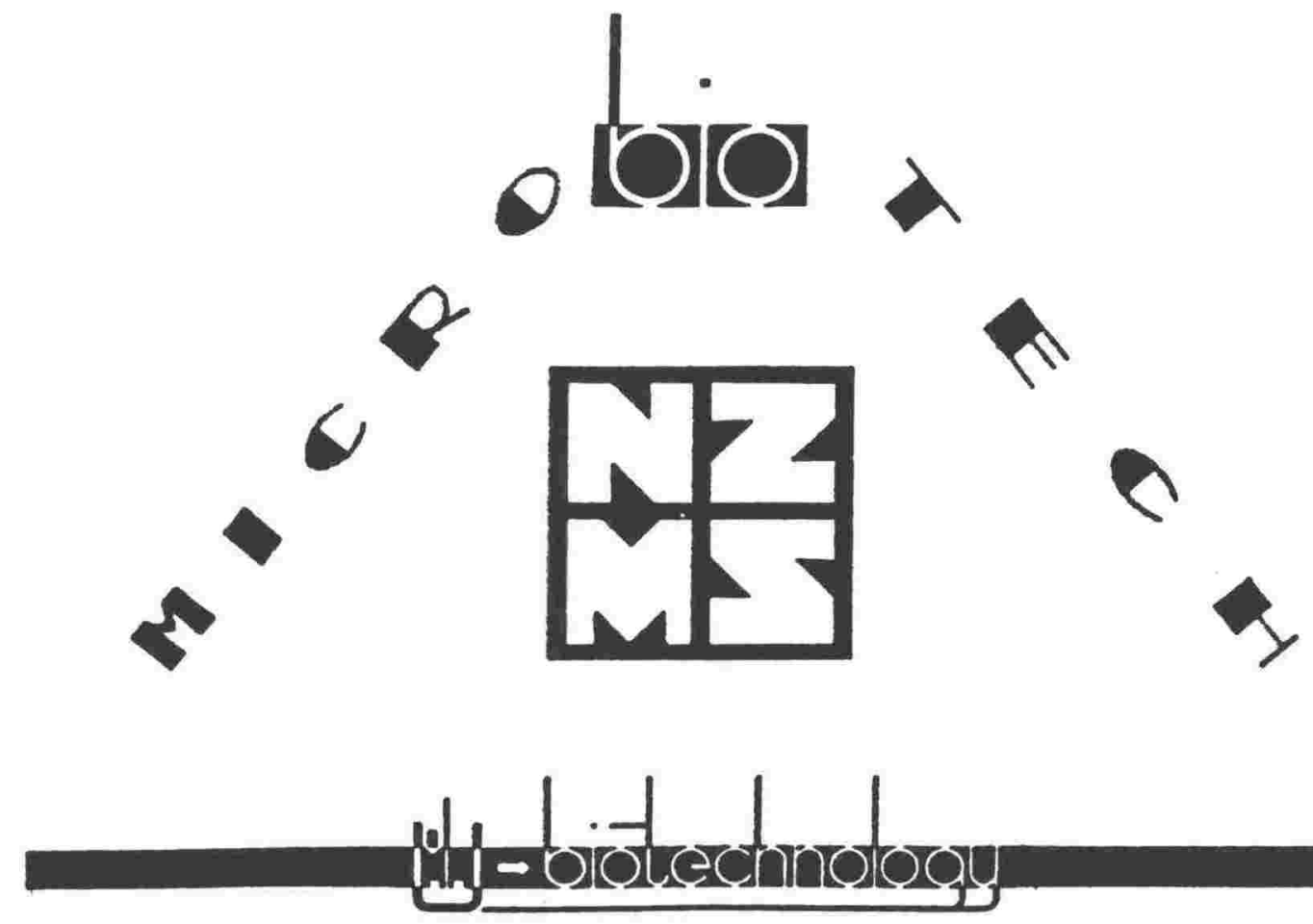

\section{PROCEEDINGS}

OF THE COABIMED

29th H.8. MICROBIOLOGICA SOCIETY COMPARE:CB and

17th 1.8. BIOTBCrinOIOGY COMPERAics

at

MASSEY UIVERSTY, PAIMERSTOM HORTE

Hay 27 - 30, 1985 .

EDITOR

MCRAET BAXTER

Department of Microbiology Genetics

Massey University 
THE EFFECT OF BIOCIOES ON MICROBIOLOGICAL ERONTHS IN MIDOLE DISTILLATE FUEL

G. Hettige and J.E. Sheridan

Botany Department, Victoria University of Wellington, Wellington

Problens involving blockages and fuel gauge failures have occurred in diesel fuelled Mew Zealand Mavy ships, "The Bounty" (pers. comm.), and in Auckland Regional withority buses [1]. In a survey carried out during 1982-83 the contaminating fungi in N.Z. navy storage tanks were identified and documented [3]. The predominant contaminant was Cladosporium resinae as in kerosene type jet fuel [8]. Penicillium Spp. and Paecilomyces variotif were also frequently isolated. Bacteria occurred infrequently.

Cladosporium resinae is generally considered to be the most troublesome contaminant of jet and diesel fuel [2]. Laboratory growth studies, however, indicate that $\underline{P}$. variotii and Penicillium spp. could produce mycel fal growths similar to $\mathrm{C}$. resinae at the fuel/water interface. Efforts should, therefore; be made to control these in addition to $\underline{C}$. resinae.

There are several lines of approach to preventing microbiological growths in fuel systems: Good housekeeping, addition of biocides and use of tank linings. A combination of all three is probably the best approach. Good housekeeping and biocides can be used to minimize growth, and tank linings can minimize corrosion should contamination occur. Good housekeeping primarily involves renoval of free water from ground storage tanks and on-board ship tanks thereby minimizing the likel thood of microbial growths becoming established. However, in underground fuel installations such as those of N.Z. navy at Devonport, free water cannot be entirely removed because of the design of the system. Inevitably, water is present and the use of biocides must be considered. corrosion is not a problem because the wall of the underground storage tanks are made of concrete. It could be a probiem, however, further downstream particularly in tank ships.

The addition of biocides to jet fuel has been widely studied $[5,7]$ but little work has been done on diesel fuel. The choice of a fuel biocide is restricted due to stringent specifications enforced by engine manufacturers. According to HIII [4] the desirable properties of a biocide are as follows: It must (1) be combustible, with no residual ash, (2) not interfere with the fuel properties on the combustion process, or any other aspect of engine performance, (3) be soluble (or very miscible) in fuel but preferentially soluble in water, (4) present no health hazard during handling or combustion, (5) not be corrosive, (6) preferrably achieve a total ' $k i 1 l^{\prime}$ of micro-organisms at minimum dose. To this list could be added (7) it must be enviromentally acceptable (where seawater is used for displacement). No such biocide has been developed for commercial use. However, a few biocides have achieved market penetration, although deficient in some of the listed characteristics. These are Biobor JF, EGME and DEGME widely used in jet fuel.

The present study involves attempts to control microbiological growth in W.2. navy underground tanks at Devonport where physical methods of control have become impossibile. The effects of commercially available (EGME, DEGME, Biobor $\mathrm{JF}$ ) and experimental biocides (Proxel AS, DML-7, Kathon 886, benomyl and inazali1) on microbiological growths in diesel fuel were studied in both laboratory and field. All the available products were screened against $C_{\text {. }}$ resinae, pectlomyces variotil and Penicillium Spp. So far oniy DEGME, Biobor, proxel IS, and bir-7 have been studied in the freld. 
1. Laboratory Tests

(a) Poison plate test:- The biocides were incorporated into $2 \%$ moiten malt agar (MEA) mis at $50^{\circ} \mathrm{C}$ at concentrations of $0,10,50,100,300 \mathrm{ppm}(0.001-0.03 \%)$, but EEME and DEEME at $0,1000,2000,3000,4000,5000 \mathrm{ppm}(0.1-0.5 \%)$. The units of measureant commonly used are $\mathrm{ppm}$ and $\%$, as in the literature and not the metric unit $\mu \mathrm{O} / \mathrm{ml}$. $5 \mathrm{~mm}$ dianeter plugs of the fungal growth were obtained from two week old cultures on MEA media and were placed centrally in each plate. The diameters of the colonies were measured after 24 hrs and subsequently at dally intervais upto 7 days. Five replicates were made for each concentration.

(b) Liquid culture test:- The activities of the biocides were tested against the mixed inoculum of the predominant fungi in varying concentrations of BushnellHaas mineral medium $(B / H)$ and sterile diesel fuel.

$B / H$ medium was prepared according to the method of Parbery and Thistlethwaite [6]. The diesel fuel was sterilized by millipore filtration $(0.22 \mathrm{um})$ and layered over the $\mathrm{B} / \mathrm{H}$ medium. The ratio of $\mathrm{B} / \mathrm{H}$ fuel and fuel:B/H were $5: 0$, $5: 1,5: 2,5: 3,5: 4$ and $5: 5$. The concentrations of benomyl, DML-7, Imazal11, Kathon 886, Proxel AS used were 0,50, 200, 300 ppm. Biobor JF concentrations were $0,125,170$ and $270 \mathrm{ppm}$ (comercially recomnended) DEGME and ECME were 0 , 1000,2000 , and $3000 \mathrm{ppm}$.

The conidial suspensions were prepared from two week old cultures grown on $V-8$ juice agar at $25^{\circ} \mathrm{C}$. B/H medium $\left(20 \mathrm{~cm}^{3}\right.$ containing $0.1 \%$ Tween 80 to aid dispersion of spores) was shaken over the $V-8$ juice agar culture and the resulting suspension centrifuged and resuspended in $\mathrm{B} / \mathrm{H} .0 .1 \mathrm{~cm}^{3}$ of the spore suspension was inoculated into $\mathrm{B} / \mathrm{H}$ :fuel containing the biocides.

The tests were done in $200 \mathrm{~cm}^{3}$ medicine flat bottles (sterilized in a hot oven at $200^{\circ} \mathrm{C}$ for $3 \mathrm{hrs}$ ). The bottles were incubated in the laboratory for six weeks. At the end of the growth period, the growth was evaluated visually.

\section{Field Test}

Biobor JF and DEGME were added at concentrations of $270 \mathrm{ppm}$ and $3000 \mathrm{ppm}$ respectively DML-7 and Proxel AS at $300 \mathrm{ppm}$ to small uncoated steel tanks at Devonport containing 25,500 litres of diesel fuel. An untreated check tank was also used. Samples were collected into sterile preserving jars and tested fortnightly for over 3 months.

\section{RESULTS \\ 1. Laboratory Tests \\ Imazalil prevented growth of $C$. resinae and Penicillium spp. at 50 ppm but} not Paecilonvces variotif. Kathon 886 was effective on all contaninating fungal growth at $50 \mathrm{ppm}$ and benomyl at $10 \mathrm{ppm}$. Dill-7 and Proxel AS was completely biostatic on the predominant fungi at $300 \mathrm{ppm}$. They inhibited the formation of mycelial mats but viable spores were present. Biobor JF, EGME and DEGME had little effect on mycelial growths regardless of the concentration (table 1).

\section{Field Test}

SIgn test showed that DEGME and Biobor JF significantly reduced $C$. resinae $(p<0.01)$, but had little effect on $P$. variotil and Penicillium spp. Ttable 2). Proxel AS and DML-7 controlled C. resinae and Penicillium Spp. but had little

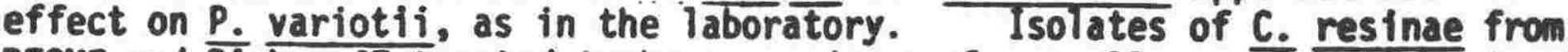
DEGME and Biobor JF treated tanks grew vigorously at all concentrations in the laboratory. 
- Table 1. Grouth of Cladosporium resinae. Paecilomyces variotif on agar plates containing different concentrations of biocides (at 8 days)

(laboratory tests).

Concentration Cladosporium resinae Peecilonyces variotif Penfcill lum Spp. of biocides

(ppm)

$0 \quad 10.50 .100 \quad 200 \cdot 300 \quad 0 \quad 1050 \quad 100 \quad 200300 \quad 0.10 .50100200300$

Benomyl

Biobor JF

DEEME*

DiL -7

EEME

Imazali

Kathon 886

Proxel AS

$\begin{array}{llllllllllllllllll}3 & 0 & 0 & 0 & 0 & 0 & 3 & 0 & 0 & 0 & 0 & 0 & 3 & 0 & 0 & 0 & 0 & 0 \\ 3 & 3 & 3 & 3 & 3 & 3 & 3 & 3 & 3 & 3 & 3 & 3 & 3 & 3 & 3 & 3 & 3 & 3 \\ 3 & 3 & 3 & 3 & 3 & 3 & 3 & 3 & 3 & 3 & 3 & 3 & 3 & 3 & 3 & 3 & 3 & 3 \\ 3 & 3 & 3 & 2 & 1 & 1 & 3 & 3 & 3 & 3 & 3 & 3 & 3 & 3 & 3 & 2 & 1 & 1 \\ 3 & 3 & 3 & 3 & 3 & 3 & 3 & 3 & 3 & 3 & 3 & 3 & 3 & 3 & 3 & 3 & 3 & 3 \\ 3 & 2 & 1 & 0 & 0 & 0 & 3 & 3 & 2 & 2 & 2 & 2 & 3 & 1 & 0 & 0 & 0 & 0 \\ 3 & 1 & 0 & 0 & 0 & 0 & 3 & 1 & 0 & 0 & 0 & 0 & 3 & 2 & 1 & 0 & 0 & 0 \\ 3 & 3 & 3 & 2 & 2 & 1 & 3 & 3 & 3 & 3 & 3 & 3 & 3 & 3 & 3 & 2 & 2 & 1\end{array}$

0 - no growth; 1 - a little growth $(6-10 \mathrm{~mm}) ; 2$ - moderate growth (11-30 mm);

3 - profuse growth $(31-80 \mathrm{~mm}) ; *-1000,2000,3000,4000$ and 5000 ppm.

Table 2. The effect of the biocides DEGME and Biobor JF on microbiological growth in diesel fuel field trials at Devonport (Apri] 1984-August 1984). Mean number of colony forming units per litre diesel fuel.

\begin{tabular}{cccc}
\hline Oate tested & $\begin{array}{c}\text { Untreated check } \\
\text { (Tank 4) }\end{array}$ & $\begin{array}{c}\text { DECME } \\
\text { (Tank 5) }\end{array}$ & $\begin{array}{c}3000 \text { ppm) } \\
\text { (Tank 6) }\end{array}$ \\
\hline
\end{tabular}
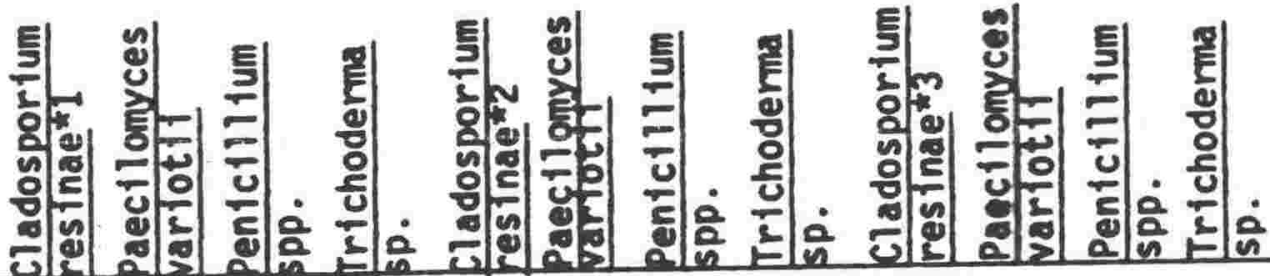

\section{April 1984}

9 May 1984

76

$67 \quad 0 \quad 45$

25 May 1984

16 June 1984

23 June 1984

17 July 1984

27 July 1984

10 August 1984

28 August 1984
39

$\begin{array}{llll}342 & 25 & 78 & 780\end{array}$

$\begin{array}{llll}106 & 26 & 26 & 296\end{array}$

$\begin{array}{llll}29 & 133 & 37 & 119\end{array}$

$\begin{array}{llll}78 & 10 & 107 & 166\end{array}$

$\begin{array}{lll}28 & 667 & 85\end{array}$

$\begin{array}{lll}18 & 1 & 18\end{array}$

$\begin{array}{lll}223 & 362 \quad 66\end{array}$
21

1136

315

$\begin{array}{lllll}441 & 0 & 254 & 92 & 230\end{array}$

$\begin{array}{lllll}107 & 4 & 6 & 11 & 111\end{array}$

14

41107

51

0

645762

$\begin{array}{lll}0 & 242\end{array}$

$\begin{array}{lll}14 & 840 & 74\end{array}$

$\begin{array}{lllll}14 & 840 & 74 & 1\end{array}$

$\begin{array}{llll}1 & 7 & 1 & 0\end{array}$

$\begin{array}{llll}2 & 542 & 40 & 100\end{array}$

Using sign test $\star_{2}$ and 3 show a significant reduction in relationship to *1 $(p<0.01)$. 
Engine tests were carried out on all the field tested biocides at Auckland, before incorporating the products into bulk fuel. The engine tests have shown Biobor JF, DEEME, Proxel AS and D.L-7 to be acceptable (0.S.E. pers. com. ).

\section{DISCUSSION}

Benonyl. Inazalil and Kathon 886 were the most effective of the biocides tested in the laboratory. Imazalil, however, had no effect against $P$. variotil. These have not yet been tested in the field. DEGME and Biobor JF performed poorly in the laboratory tests but inhibited $C$. resinae temporarily in the field. The reason for this is unknown. However, the $C$. resinae isolates from DEGME and Biobor JF tanks grew vigorously when tested in the laboratory. Dil -7 and Proxel AS performed well against $C$. resinae and Penicillium SpP. . In both the laboratory and field tests, but were Tess effective against P. variotif. Laboratory studies showed that $P$. variotif and Penicillium SPD. are capable of forming thick mycelial mats at the fuel/water interface. Hence it is important to control these growths in the storage tanks.

There are large fluctuations in the numbers of colonies recovered from the three storage tanks. This could be due to a number of factors:- clumping of fungal mycelium and spores, settling effect, interaction among the dominant fungi, seasonal and biocide effects. Studies are currently underway to determine the cause of the large fluctuation encountered.

1. Anon (1981). Fungus stops buses. Evening Post, 3rd Apri1 1981.

2. Hendey, N.I. (1964). Some microbiological problems encountered in naval sciences. Corrosion symposium of the Commonwealth Defence Science Organisation.

3. Hettige, G. and Sheridan, J.E. (1984). Mycoflora of stored diesel fuel in New Zealand. International Biodeterioration Bulletin 20(4): 225-228.

4. Hill, E.C. (1982). Preservation in specialised areas. B. Fuels and Lubricants. In Principles and Practice of Disinfection. Preservation and Sterilization. (Ed. Russell, A.D., Hugo, M.B. and Avliffe, G.A.J.) pp. 352-357. London: Blackwell Scientific Publishers.

5. Hitzman, D.O., Shotton, J.A. and Alquist, H.E. (1963). Biocidal Effects of PFA $55 \mathrm{MB}$. Society of Autonotive Engineers and American Society of Naval Engineers, Joint Kational Aeronautical Keeting. Paper SAE 6830, Washington D.C. ApriT 8-11.

6. Parbery, D.G. and Thistlethwaite, P.J. (1973). Improved mineral salts solution for growth of Anorphotheca resinae. International Biodeterior ation Bulletin 9: 11-16.

7. Rogers, M.R. and Kaplan, A.M. (1968). Screening of prospective biocides for hydrocarbon fuels. Developments in Industrial Microblology 9: 448-77 (Socfety for Industrial Nicrobiology).

8. Sheridan, J.E. (1972). Studies on the kerosene fungus Amorphotheca resinae in New Zealand. International Biodeterioration BuTletin T2(I): $31-33$.

\section{ACKNOMLEGEMENTS}

The authors wish to thank the staff of the Defence Scientific Establishment, Auckland, for their help and assistance, especially Mr H. Levinsohn and Mr M. Cavanaugh. This study was financed by research grant from the Hinistry of Defence which is much appreciated. 


\title{
MYCOFLORA OF STORED DIESEL FUEL IN NEW ZEALAND
}

\section{G. HETTIGE ${ }^{1}$ and J.E. SHERIDAN ${ }^{1}$}

\begin{abstract}
Twelve monthly samples of diesel fuel from two bulk storage tanks were examined for the presence of fungal contamination during 1982-1983. Over 25 fungal species were isolated.

"Cladosporium resinae was the predominant fungus occurring in $98 \%$ of the samples, followed by Penicillium spp. (93\%). A list of fungi identified and frequency of isolation is given.
\end{abstract}

\section{Introduction}

Microbial contamination of diesel fuel in storage tanks, shipboard fuel tanks and locomotives has been studied by various workers: Stormont (1962), Zeitlow \& Hostetler (1962), Flippin, Smith \& Mickelson (1964), Wright \& Hostetler (1963), Hendey (1964), Neihof (1980), May \& Neihof (1981), Turner (1981) and Bruce (1982), but not as widely as for jet fuel.

Cladosporium resinae has been reported as the major contaminant of diesel although recently Bruce (1982) observed Penicillium spp. in the fuel/water system and suggested that they might be as important colonizers as $C$. resinae. Whether Penicillium spp. are as harmful as $C$. resinae is not yet established.

The corrosive activity of $C$. resinae on aluminium-copper alloy and stainless steel in the diesel/water system was studied by Hansen, Tighe-Ford and George (1981). They found that stainless steel was not attacked but significant corrosion occurred on aluminium-copper alloy.

In New Zealand, major problems with diesel fuel blockages and fuel gauge failures have occurred in navy ships, "The Bounty" (pers. comm.), and in buses (news reports - see Anon 1981) but little work has been carried out to establish the main cause of the problem.

Cladosporium resinae is known to be widespread in aircraft fuel, in soils and airspora in New Zealand (Sheridan 1972) but a survey of microbial contamination in diesel fuel systems in this country has not been made. No publication has actually documented the fungi found in stored diesel fuel.

In the studies reported here fungi were identified in monthly samples of diesel fuel collected from two concrete storage tanks sited in Auckland during 1982-83. A list of fungi identified is given together with the numbers and frequency of isolation.

\section{Materials and methods}

\section{Sampling techniques}

Fuel from the top, middle and bottom of the storage tanks was sampled by a weighted sampling can. shown in Fig. 1a. This is a brass bottle with a fuel holding capacity of one litre, which fills with fuel after being lowered to the appropriate level when the stopper is removed by the captive rope.

\section{Fig. 1a. Samplers}

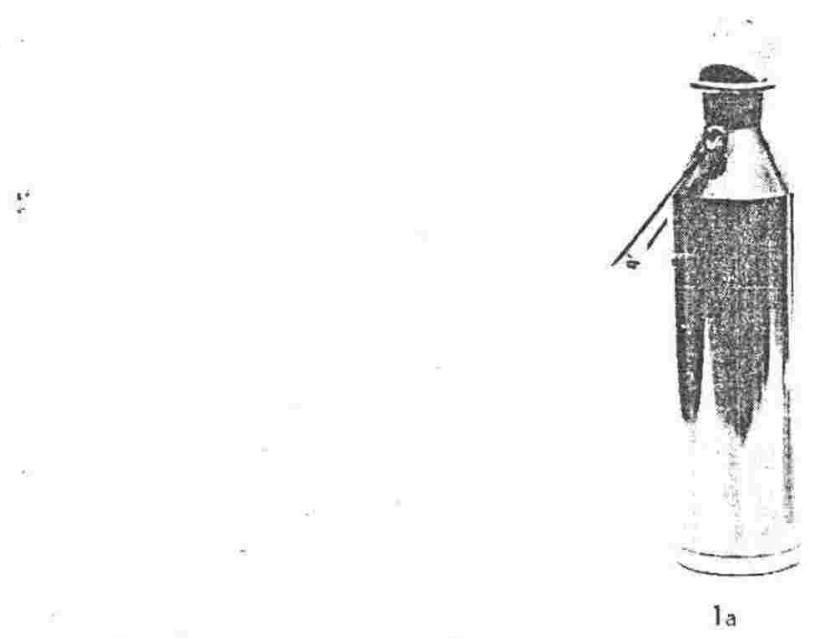

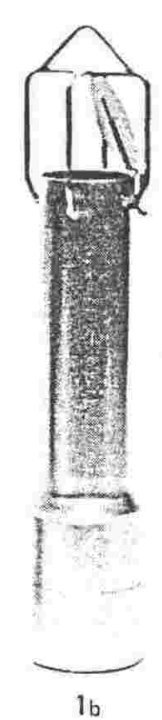

Ib

The 'very bottom' of the tank was sampled by a 'zone sampler' also sometimes called the 'thief' as in Fig. $1 \mathrm{~b}$. The sampler allows the fuel to pass through as it descends through the fuel in the tank. This is made possible by the top and bottom valves opening due to the pressure of the fuel against the descending sampler. When the sampler becomes stationary, the valves close and the sample is retained as the sampler is returned to the surface.

The fuel samples were immediately transferred into clean, sterile, glass preserving jars in such a manner as to minimize contamination from the environment and transported to Wellington where the microbiological examination was carried out. The sampling bottles and the glass preserving.jars were washed in detergent. rinsed with $95 \%$ alcohol and sterilized at $200^{\circ} \mathrm{C}$ in a hot oven for three hours before collection of the diesel fuel. During 1982-83 twelve monthly samples of diesel fuel were collected from each of two concrete storage tanks using the sterile samplers described above.

${ }^{1}$ Botany Department, Victoria University of Wellington. New Zealand. 
Microbiological procedures

Fungal assays were carried out on the samples both immediately and 24 hours after sampling. Later this was extended to $4-5$ days to observe any changes in the composition and relative abundance of the mycoflora with time. Because the time of testing of samples after collection did not appear to be critical, immediate testing in Auckland was discontinued.

$100 \mathrm{ml}$ of each sample, after thorough shaking, was filtered through a $0.45 \mu \mathrm{m}$ millipore membrane filter. V-8 juice agar at $\approx 45^{\circ} \mathrm{C}$ was poured over the filter membrane in a sterile Petri dish. The plate was incubated at $25^{\circ} \mathrm{C}$ and examined after 5 days. $100 \mathrm{ml}$ of each sample was filtered separately and the membrane filter examined directly under phase contrast to obtain some idea of the spore types present and compare with viable cultures. All tests were carried out in duplicate.

In growth experiments, Cladosporium resinae, Penicillium spp. and Paecilomyces variotii were grown separately and as mixed fungal inoculum in sterile diesel fuel/Bushnell-Haas mineral salts medium $(B / H)$. The ratio of fuel:B/H used was $1: 1$. The diesel fuel was sterilized by millipore filtration $(0.22 \mu \mathrm{m}$ millipore membrane filter). The conidial suspensions were prepared from two week old cultures grown on V-8 juice agar at $25^{\circ} \mathrm{C} .20 \mathrm{ml}$ of $\mathrm{B} / \mathrm{H}$ medium was shaken over the $\mathrm{V}-8$ juice agar culture and the resulting suspension centrifuged in a $15 \mathrm{ml}$ sterile glass centrifuge tube at $1520 \mathrm{xg}$ for $20 \mathrm{mins} .0 .1 \mathrm{ml}$ of the above fungal spore suspensions were inoculated into the fuel:B/H (approximately 500,000 spores $/ \mathrm{ml}$ ). The tests were set up in $200 \mathrm{ml}$ medicine flat bottles (sterilized in an oven at $200^{\circ} \mathrm{C}$ for 3 hours). The aluminium caps with the rubber liner removed, were screwed on loosely and the bottles incubated at $25^{\circ} \mathrm{C}$ for six weeks. At the end of the growth period, growth was assessed visually. Where there appeared to be little or no growth the contents of the bottles were filtered through $0.45 \mu \mathrm{m}$ membrane filters, partially wet in sterile water and diesel fuel. Lactophenol cotton blue was applied to the filters which were then observed microscopically.

The airspora in the immediate vicinity of the storage tank was monitored by.a Kramer-Collins 7-day spore sampler (Kramer et al. 1976).

\section{Culture Media}

The V-8 juice agar was prepared as follows. 20 grams of Davis agar (other brands should also be suitable) was added to $800 \mathrm{ml}$ tap water in a glass vessel which was steamed until the agar melted. To this was added $200 \mathrm{ml}$ of V-8 juice (Campbell's Soups Ltd.). The medium was mixed gently, distributed into medicine flats and autoclaved at $103 \mathrm{kPa}$ (15 p.s.i.) for 10 minutes. Bushnell-Haas mineral salts medium was prepared according to the method of Parbery \& Thistlethwaite (1973).

\section{Fungal Identifications}

Fungi were examined microscopically and identified using available keys. The Commonwealth Mycological Institute, Kew, England, identified or confirmed identification of a number of species; these are indicated by an asterisk in Table 1.

Table 1. Fungi isolated from New Zealand stored diesel fuel 1982-83.

\begin{tabular}{|c|c|c|c|c|c|}
\hline \multirow[t]{2}{*}{ Name of Fungus } & \multicolumn{4}{|c|}{$\begin{array}{l}\text { Mean number of colony } \\
\text { forming units/litre }\end{array}$} & \multirow[t]{2}{*}{$\begin{array}{l}\text { Percentage } \\
\text { frequency } \\
\text { of isolation }\end{array}$} \\
\hline & Top & Middle & Bottom & $\begin{array}{l}\text { Very } \\
\text { Bottom }\end{array}$ & \\
\hline \multicolumn{6}{|l|}{ Cladosporium resinae (Lindau) de } \\
\hline Vries & 40 & 160 & 972 & 998 & 98 \\
\hline Penicillium corylophilum* Dierckx & 50 & 43 & 473 & 550 & $40 \mid$ \\
\hline \multicolumn{6}{|l|}{ Penicillium digitatum ${ }^{*}$} \\
\hline (Pers. ex Fr.) Sacc & 1 & 3 & 55 & 200 & 10 \\
\hline Penicillium echinulatum* & & & & & 93 \\
\hline Raper \& Thom ex Fassatiova & 3 & 8 & 28 & 70 & 10 \\
\hline Penicillium frequen tans* Westling & 7 & 12 & 15 & 25 & 23 \\
\hline Penicillium spinulosum* Thom & 10 & 9 & 10 & 13 & 10) \\
\hline Paecilomyces variotii Bainier & 3 & 8 & 300 & 425 & 50 \\
\hline Fusarium spp. & 4 & 8 & 17 & 24 & 30 \\
\hline Mucor sp. & 0 & 10 & 23 & 11 & 26 \\
\hline \multicolumn{6}{|l|}{ Paecilomyces lilacinus* (Thom) } \\
\hline Samson & 0 & 10 & 13 & 7 & 24 \\
\hline \multicolumn{6}{|l|}{ Alternaria alternata $(\mathrm{Fr})}$. \\
\hline Keissler & 4 & 9 & 18 & 0 & 23 \\
\hline Trichoderma harzianum* Rifai & 12 & 3 & 1 & 0 & 18 \\
\hline \multicolumn{6}{|l|}{ Cladosporium cladosporioides } \\
\hline (Fresen.) de Vries & 5 & 9 & 2 & 0 & 17 \\
\hline Rhizopus sp. & 5 & 6 & 0 & 0 & 15 \\
\hline Chaetomium globosum Kunze ex Fr. & 7 & 5 & 0 & 0 & 14 \\
\hline Altenaria sp. & 4 & 8 & 0 & 0 & 13 \\
\hline \multicolumn{6}{|l|}{ Aureobasidium pullulans (de Bary) } \\
\hline Arnaud & 6 & 1 & 2 & 1 & 12 \\
\hline \multicolumn{6}{|l|}{ Aspergillus sejunctus* Bainier } \\
\hline and Sartory & 0 & 13 & 5 & 0 & 11 \\
\hline Yeast & 0 & 1 & 10 & 17 & 10 \\
\hline Unidentified & 2 & 5 & 4 & 1 & . 9 \\
\hline \multicolumn{6}{|l|}{ Aspergillus versicolor* (Vuill.) } \\
\hline Tiraboschi & 0 & 9 & 0 & 0 & 8 \\
\hline Phomopsis sp. & 3 & 8 & 0 & 0 & 7 \\
\hline Aspergillus niger Van Tiegh & 0 & 23 & 0 & 18 & 5 \\
\hline \multicolumn{6}{|l|}{ Epicoccum purpurascens } \\
\hline Ehrenb, ex Schlecht & 5 & 3 & 0 & 0 & 4 \\
\hline Gliocladium roseum Corda & 4 & 2 & 0 & 0 & 3 \\
\hline Ulocladium sp. & 4 & 1 & 0 & 0 & 3 \\
\hline
\end{tabular}

*Identified or identification confirned by the Commonwealth Mycological Institute. Kew, England.

Quantitative estimation of the level of contamination in the tanks was made by recording the number of samples in which a particular fungus occurred and the number of colony forming units of the fungus. 
Results

A list of fungi showing numbers and frequency of isolation is given in Table 1 . All of the monthly samples tested were contaminated with microorganisms. The predominant fungus was C. resinae isolated from $98 \%$ of the samples. Penicillium spp. were isolated from $93 \%$ and Paecilomyces variotii from $50 \%$ of the samples. The highest number of colony forming units (cfu) in the samples was obtained for C. resinae where almost 1000 cfu/litre occurred in the bottom and 'very bottom' samples. All the above fungi grew vigorously in diesel fuel/Bushnell-Haas mineral salts medium at $25^{\circ} \mathrm{C}$, forming a thick mycelial mat at the interface.

Among the five Penicillium spp., Penicillium corylophilum had the highest number of colony forming units in the diesel fuel. The Penicillium spp. produced separate distinctive and individual colonies on the media.

It appeared from microscopic observations and growth experiments in diesel fuel/B/H that Penicillium spp. remain as spores in the presence of $C$. resinae.

During the period when Penicillium spp. were observed in high numbers in the atmosphere, there was a corresponding increase in numbers in the fuel. Alternaria alternata occurred in abundance on filters observed directly but was infrequently isolated as colonies from the filters on agar plates.

\section{Discussion}

Over 25 fungal species were isolated from stored New Zealand diesel fuel during a twelve month period in $1982-83$. The most frequently isolated fungi were Cladosporium resinae, Penicillium spp. and Paecilomyces variotii, as in jet fuel (Sheridan \& Soteros 1974). There was a gradient in contamination levels of $C$. resinae, Penicillium spp. and Paecilomyces variotii with the highest concentration at the bottom and 'very bottom'. This was probably due to the greater proportion of water present at these levels.

A 24 hour delay in testing samples after collection and in transportation had little effect on the recovery of the above fungi.

Penicillium spp. generally accompanied C. resinae but only the latter formed tough mats of mycelium. Growth experiments indicated some form of interaction between Penicillium spp. and $C$. resinae possibly similar to that suggested by Neihoff (1980) for Candida albicans and C. resinae in seawater systems. Aspergillus fumigatus was not isolated from diesel fuel in these studies, although it occurred in New Zealand jet fuel (Sheridan \& Soteros 1974).

Microbial interactions on the culture media plates could result in antagonism or masking of some fungi. Direct observations of filters revealed a wide variety of fungal spore types in stored diesel fuel. Airspora studies suggest that many such as Alternaria, Epicoccum, Penicillium, Ulocladium and others could have entered the tanks from the atmosphere.

The mycoflora of New Zealand diesel fuel cannot be compared with that of other countries because of unavailability of published data. To our knowledge this is the first report to document fungi found in diesel fuel.

The authors wish to thank the staff of the Defence Scientific Establishment, Auckland, for their help and assistance, especially Mr H. Levinsohn and $\mathrm{Mr}$ M. Cavanaugh. This study was financed by a research grant from the Ministry of Defence, which is much appreciated.

\section{References}

Anon (1981) Fungus stops buses. Evening Post, 3rd April 1981.

Bruce, N. (1982) A laboratory study of microbial growth in simulated fuel tanks. International Biodeterioration Bulletin 18,87-91.

Flippin, R.S.; Smith, C.; Mickelson, M.N. (1964) Fusarium growth supported by Hydrocarbons. Applied Microbiology 12, 93-95.

Hansen, D.J.; Tighe-Ford, D.J.; George, G.C. (1981) Corrosive activity of the fungus Cladosporium resinae in a Dieso/water system. Journal of Naval Science 7, 174-183.

Hendey, N.I. (1964) Some microbiological problems encountered in naval sciences. Corrosion Symposium of the Commonwealth Defence Science Organisation.

Kramer, C.L.; Eversmeyer, M.G.; Collins, T.I. (1976) A new 7 day spore sampler. Phytopathology 66, 60-61.

May, M.E.; Neihof, R.A. (1981) Growth of Cladosporium resinae in seawater/fuel systems. In Developments in Industrial Microbiology 33, $781-787$.

Neihof, R.A. (1980) Twenty-eighth conference on microbiological deterioration of military material, 13-15 November 1979. Technical Report, Natick/TR-80/018, 129-138.

Purbery, D.G.; Thistlethwaite, P.J. (1973) Improved mineral salts solution for the growth of Amorphotheca resinae. International Biodeterioration Bulletin 9, 11-16.

Sheridan, J.E. (1972) Studies on the kerosene fungus Amorphotheca resinae in New Zealand. International Biodeterioration Bulletin 12, $31-33$.

Sheridan, J.E.; Soteros, J.J. (1974) A survey of fungi in jet aircraft fuel systems in New Zealand. International Biodeterioration Bulletin 10 , 105-107.

Stormont, D.H. (1962) Boron additive helps NYC combat bacterial fouling of diesel fuel. Oil and Gas Joumal September 17.

Turner, A.P.F. (1981) Conditions affecting the growth of Cladosporium resinae in a ship fuel systems. Bulletin of British Mycological Society 15 (suppl.), 6-7.

Wright, R.H.; Hostetler, H.F. (1963) Microbiological diesel fuel contamination. Society of Automotive Engineering Congress, Detroit, Michigan, January 14-18.

Zeitlow, M.E.; Hostetler, H.F. (1962) Microbiological diesel fuel contamination. National Association of Railroad Engineers Tests, Spring meeting, Chicago, Illinois. 